\title{
The Synthesis and Characterisation of Nano-structured Calcium Silicate
}

\author{
By \\ Andrew James McFarlane
}

A thesis

submitted to the Victoria University of Wellington

in fulfilment of the requirements for the degree of

Doctor of Philosophy

in Chemistry

Victoria University of Wellington 


\section{Abstract}

Nano-structured calcium silicate consists of randomly stacked nano-sized platelets that make up an open framework structure of macropores that resembles a house of cards. This structure affords the material the desirable physical properties of a large pore volume and a highly accessible surface area that exceed many other silicas and silicates. The material is possibly related to other disordered calcium silicate hydrates at an atomic level, although it is the macro-structure and the potential of performing chemistry upon its surface that is of great interest.

Due to the novelty of nano-structured calcium silicate, little was known about it before this work. The focus of this study has therefore been placed upon characterising the material and determining the conditions that allow the pore volume and surface area to be maximised. The material is prepared through an initial precipitation from the reaction of a calcium salt with monomeric silica, followed subsequently by self-ordering on both an atomic-scale and on a macro-scale to develop the porous framework. The framework of the material has been found to collapse due to forces created from surface tension during the removal of water from the pores upon drying. The result of this collapse is a substantial reduction in both the surface area and pore volume of the material. Three different methods have been developed to maintain the structure with each modification producing a material that is suitable for different applications. A reinforcing process following the development of the open framework whereby additional silica is polymerised upon the structure strengthens the material so that the forces resulting from the removal pore water are unable to cause collapse of the framework. This material is therefore able to be repeatedly re-wet and dried without any detrimental effect to the pore volume or surface area of the material. The replacement of water within the pores with 2-ethoxyethanol, that has a low surface tension, and by modifying the material through treatment with acid have also been found to prevent collapse of the structure. Through the knowledge gained of the development of the nano-structured calcium silicate and of the reaction 
conditions required for the optimisation of the surface area and pore volume, a semi-continuous process has been devised that has allowed for production of the material on a larger scale.

This work also contains details on the formation of nano-structured calcium silicate by using geothermal water from an electricity generation plant as the source of monomeric silica rather than using sodium silicate. Currently, the formation of a scale from supersaturated geothermal water is problematic for the industries that use the fluid and limits the use of the resource. The removal of monomeric silica from geothermal water as a result of producing nanostructured calcium silicate prevents the formation of the scale and therefore allows a greater proportion of the thermal energy in the fluid to be potentially utilised. 


\section{Acknowledgements}

There are many people that I would like to thank for their assistance and for their friendship over the course of this work. I'm not the type of person that expresses my appreciation for what you've all done for me or mean to me very often or very well, and while regrettably these brief words do not address this deficiency, I hope that these few lines give a slight indication of the gratitude that I truly feel.

I would like to thank my family for their continual and unwavering support and my supervisor Jim for his guidance and everlasting enthusiasm. I would also like to thank Thomas, Jackie, and Teresa for their assistance at the coal-face and equally if not more so for their friendship. I consider myself to have been very fortunate to have always been surrounded by so many wonderful people that made for such a pleasant work environment, and for their friendship I would like to thank Mike, Rob, Laine, Kirsten, James, Rhys, Janeen, John R, Ben, Ray, John M, all of the crew from lab 107 past and present as well as numerous other people from the department over the years. For their invaluable and always willing assistance I would also like to thank Alan, Manu, Dave G, Dave S, Rob L, Scott, Gordon, Grant, Ken, Karen, Barry, John P, Ding, and Chris.

AJM 


\section{Table of Contents}

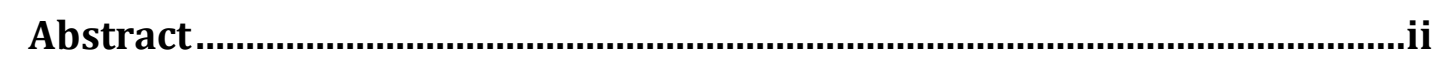

Acknowledgements......................................................................................iv

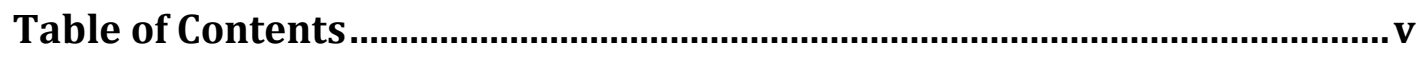

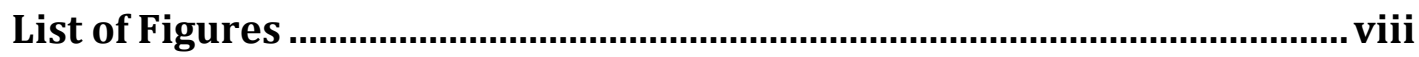

List of Tables............................................................................................ Xvi

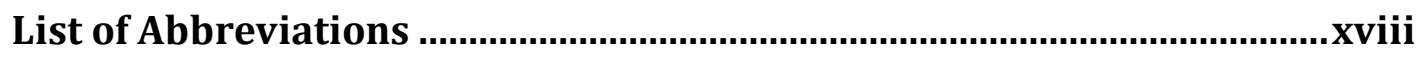

Chapter 1 - Introduction .............................................................................1

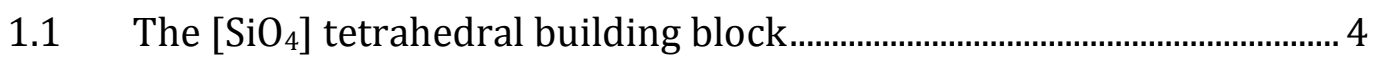

$1.2 \quad$ Silicates

1.2.1 Zeolites ……………………………………………………………………...

1.2.2 Mesoporous molecular sieves …………………………………………….

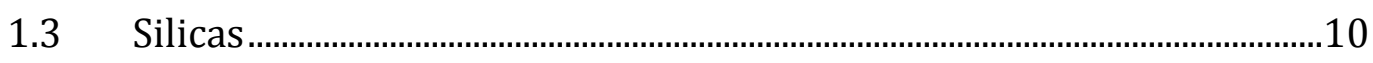

1.3.1 Colloidal silica ...........................................................................................11

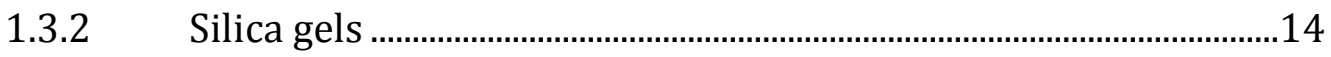

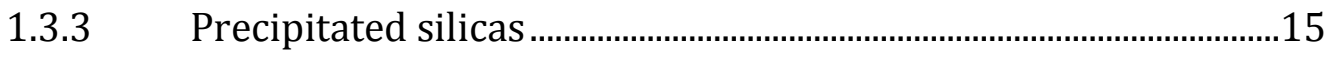

1.3.4 Pyrogenic silicas................................................................................16

1.3.5 Biogenic silicas.........................................................................................17

1.4 Macroporous materials.........................................................................................17

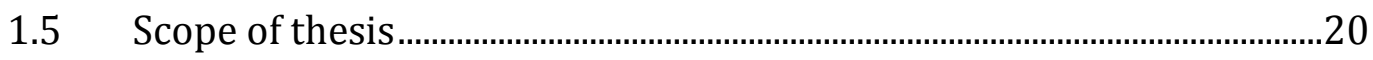

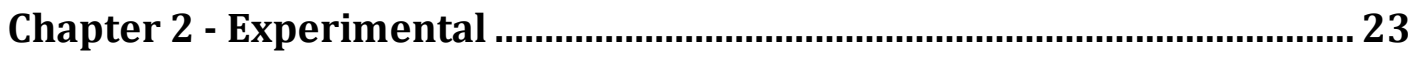

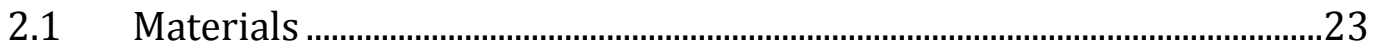

2.2 Precipitation of nano-structured calcium silicate........................................24

2.2.1 Method for batch production.................................................................24 
2.2.2 Method for continuous production

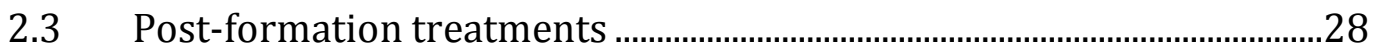

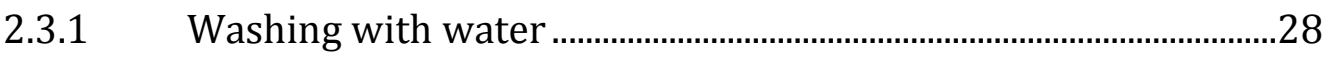

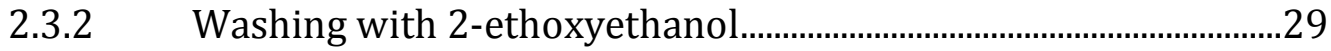

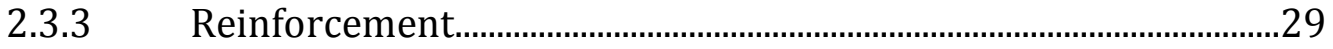

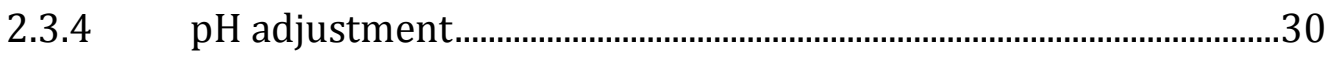

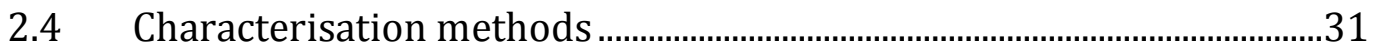

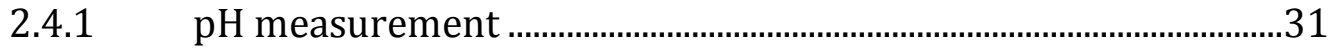

2.4.2 Monomeric silica concentration analysis.........................................31

2.4.3 Atomic absorption spectroscopy …...................................................33

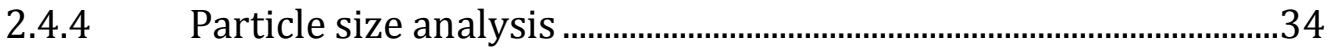

2.4.5 Transmission electron microscopy ......................................................34

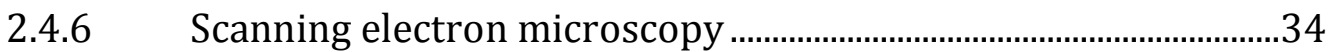

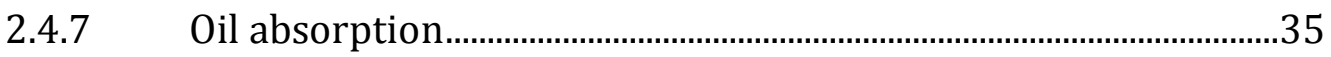

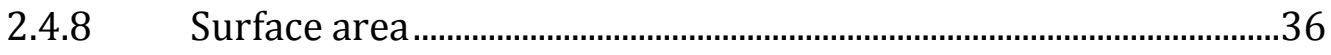

2.4.9 Thermogravimetric analysis …….........................................................38

2.4.10 Powder X-ray diffraction .....................................................................38

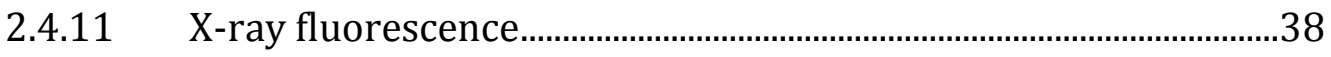

2.4.12 ${ }^{29}$ Si Nuclear magnetic resonance ..........................................................39

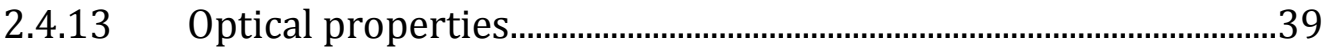

Chapter 3 - Formation of nano-structured calcium silicate ........................ 41

3.1 The precipitation process ........................................................................... 41

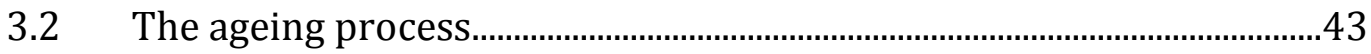

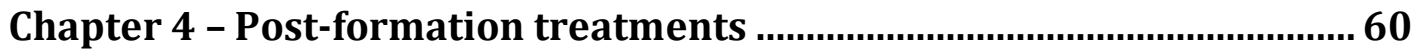

4.1 Application of 2-ethoxyethanol ..............................................................61 
4.2 Reinforcement of the structure .69

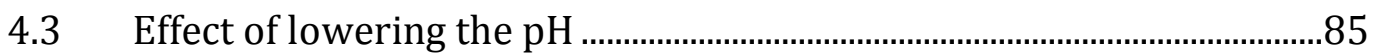

Chapter 5 - Optimisation of reaction conditions....................................100

5.1 Changing the calcium to silicon ratio ..........................................................100

$5.2 \quad$ Effect of pH upon precipitation..................................................................104

5.3 Effect of changing both calcium and hydroxide concentrations........111

5.4 Effect of temperature upon precipitation ..............................................115

$5.5 \quad$ Effect of scale and mixing ……..................................................................116

5.6 The effect of concentration ......................................................................117

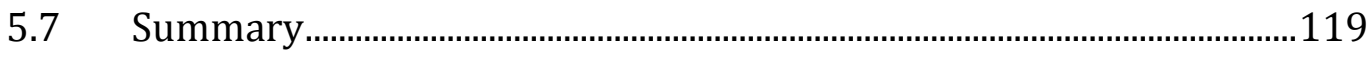

Chapter 6 - Nano-structured calcium silicate from geothermal water..128

6.1 The first series of reactions using geothermal water............................134

6.2 The second series of reactions using geothermal water ......................154

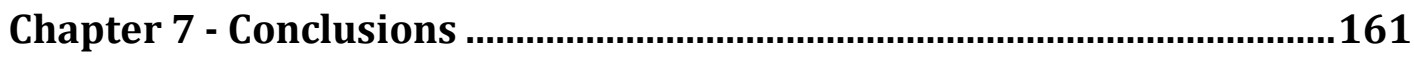

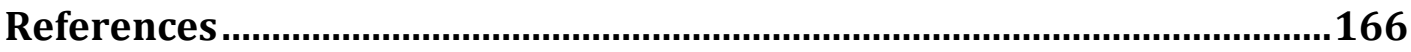

Appendix A - Data for graphs................................................................. 172

Appendix B - Supplemental geothermal sample data................................185

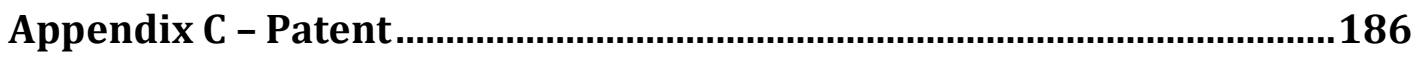




\section{List of Figures}

Figure 1.1: A scanning electron micrograph of the novel nano-structured calcium silicate revealing the porous nature of the material.

Figure 1.2: A transmission electron micrograph of a silica precipitated from geothermal water. (Meyer, 1996).

Figure 1.3: Calculated bending potentials for Si-O-Si and Si-S-Si units. (Burdett, 1988)

Figure 1.4: (a) Framework structure showing the topology of the molecular sieve ZSM-5 viewed in the direction of the main channel. (Flanigan et al., 1978) (b) A representation of the two-dimensional channel system in ZSM-5. (Kokotailo et al., 1978).

Figure 1.5: Transmission electron micrographs of MCM-41 having hexagonal pores in a regular honeycomb arrangement (Beck et al., 1992).

Figure 1.6: Solubility of amorphous silica at different temperatures over a $\mathrm{pH}$ range (Iler, 1979)

Figure 1.7: A representation of the growth of a sol particle from the start of polymerisation. (Coradin \& Lopez, 2003)

Figure 1.8: A general polymerisation curve showing the concentration of monomeric silica against time.

Figure 1.9: The polymerisation of silica with the formation of networks (A) or the growth of sol particles (B). (Iler, 1979).

Figure 1.10: A representation of the bridge formed by a cationic agent (calcium in this case) due to the cation bonding to some of the ionised silanol groups. .16

Figure 1.11: Representations of a gel network and discreet precipitated silica particles formed from the sol particles of a stable silica colloid .16

Figure 1.12: Scanning electron micrographs of biogenic silicas. (a) The concentric layers of a sponge spicule. (b) The pores in the shell of a diatom (Thalassiosira sp. frustule). (Coradin \& Lopez, 2003).

Figure 1.13: Scanning electron micrographs of mesoporous silica and its template. (Schroden \& Stein, 2004).. .18

Figure 1.14: A hierarchical material with the walls of the macropores made of mesoporous silica.. 
Figure 1.15: Scanning electron micrographs of macroporous materials with no long range order. (a) An inorganic cellular foam. (b) A cellular aragonite framework on a metal substrate..................................................................................19

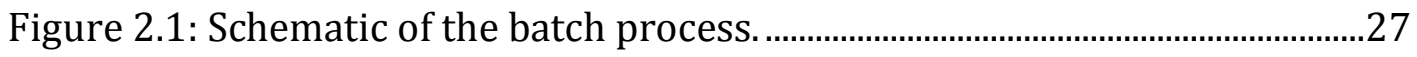

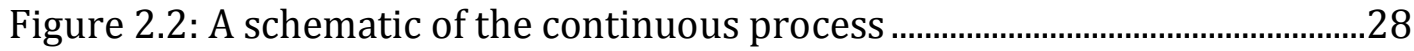

Figure 2.3: Weight gain as a result of the uptake of atmospheric moisture by nano-structured calcium silicate under typical laboratory humidity and temperature conditions.................................................................................................36

Figure 2.4: A representation of the CIELAB colour space (HunterLab, 2001) ...40

Figure 3.1: Change in the concentration of the reactants remaining in solution during the ageing process.

Figure 3.2: Composition of solids during the ageing process as determined by XRF.

Figure 3.3: Scanning electron micrograph of 2-ethoxyethanol treated nanostructured calcium silicate 2 minutes after precipitation.

Figure 3.4: Scanning electron micrograph of 2-ethoxyethanol treated nanostructured calcium silicate 10 minutes after precipitation.

Figure 3.5: Scanning electron micrograph of 2-ethoxyethanol treated nanostructured calcium silicate 60 minutes after precipitation.

Figure 3.6: Scanning electron micrograph of 2-ethoxyethanol treated nanostructured calcium silicate 150 minutes after precipitation.

Figure 3.7: Scanning electron micrograph of 2-ethoxyethanol treated nanostructured calcium silicate 300 minutes after precipitation.

Figure 3.8: A transmission electron micrograph of a fully aged nano-structured calcium silicate that has been treated with 2-ethoxyethanol.

Figure 3.9: Change in physical properties during the ageing process of materials that have been treated with 2-ethoxyethanol to prevent collapse of the structure.

Figure 3.10: Loss of volatile materials from samples taken during ageing when ignitited at $1000^{\circ} \mathrm{C}$.

Figure 3.11: Particle size changes over time during the ageing process..

Figure 3.12: ${ }^{29} \mathrm{Si}$ NMR spectra indicating the change in the atomic environment during the ageing process. 
Figure 3.13: X-ray diffractograms of nano-structured calcium silicate during ageing.

Figure 3.14: X-ray diffractograms of calcium silicate hydrates with varying compositions. (Chen et al., 2004).

Figure 3.15: Single layer of $1.4 \mathrm{~nm}$ tobermorite seen along (a) [210] and (b) [010] illustrating the Ca-O main layer with attached silicate tetrahedra dreierketten chains. (Chen et al., 2004).

Figure 3.16: The two possible layers of $1.4 \mathrm{~nm}$ tobermorite, and their symmetry elements. (Bonaccorsi et al, 2005)

Figure 3.17: Crystal structure of $1.4 \mathrm{~nm}$ tobermorite. (Bonaccorsi et al., 2005) .55

Figure 3.18: X-ray diffractograms of nano-structured calcium silicate that has been calcined at different temperatures.

Figure 3.19: Scanning electron micrograph of nano-structured calcium silicate treated with 2-ethoxyethanol that has been calcined at $700{ }^{\circ} \mathrm{C}$.

Figure 3.20: Scanning electron micrograph of nano-structured calcium silicate treated with 2-ethoxyethanol that has been calcined at $800{ }^{\circ} \mathrm{C}$ . .58

Figure 3.21: Effect of calcining nano-structured calcium silicate treated with 2ethoxyethanol at different temperatures upon the oil absorption capacity and surface area of the material.

Figure 4.1: A schematic of the chelation of 2-ethoxyethanol to surface calcium groups.

Figure 4.2: Scanning electrons micrographs of a nano-structured calcium silicate that has collapsed upon drying and maintained its structure upon drying at $110^{\circ} \mathrm{C}$ after being treated with 2-ethoxyethanol.

Figure 4.3: The effect of plug flow volume of 2-ethoxyethanol upon the oil absorption capacity.

Figure 4.4: Effect of the proportion of 2-ethoxyethanol in plug flow upon the physical properties.

Figure 4.5: The effect of 2-ethoxyethanol upon the weight of dried product...64

Figure 4.6: Thermogravimetric analysis of a nano-structured calcium silicate that had been washed with 2-ethoxyethanol and the same material washed only with water. 
Figure 4.7: A schematic of the experimental set-up for exposing nanostructured calcium silicate to constant relative humidities

Figure 4.8: Weight gain of nano-structured calcium silicate due to the adsorption of water under $75 \%$ and $29 \%$ constant relative humidity conditions relative to the materials weight when dried at $110{ }^{\circ} \mathrm{C}$. .68

Figure 4.9: The effect of treating nano-structured calcium silicate with 2ethoxyethanol upon the whiteness and brightness of the material.

Figure 4.10: The effect of treating nano-structured calcium silicate with 2ethoxyethanol upon the colour of the material.

Figure 4.11: Variation in solubility of silica with radius of curvature of surface (Iler, 1979)

Figure 4.12: A representation of two intersecting nano-structured calcium silicate plates before and after reinforcement.

Figure 4.13: Effect of reinforcement upon the oil absorption capacity.

Figure 4.14: The concentration of monomeric silica remaining in solution following reinforcement.

Figure 4.15: The effect of reinforcement upon the composition of the resulting nano-structured calcium silicate.

Figure 4.16: X-ray powder diffractograms of nano-structured calcium silicate before and after reinforcement. .75

Figure 4.17: Concentration of unreacted monomeric silica remaining in solution during the reinforcement reaction.

Figure 4.18: Development of the oil absorption capacity and surface area during the reinforcement reaction.

Figure 4.19: Effect of reinforcement upon physical properties at ca. $\mathrm{pH} 10 \ldots . . .78$

Figure 4.20: Monomeric silica remaining in solution following reinforcement at ca. $\mathrm{pH} 10$.

Figure 4.21: Increase in product weight due to reinforcement.

Figure 4.22: The effect of reaction $\mathrm{pH}$ upon the oil absorption capacity of nanostructured calcium silicate reinforced with different amounts of monomeric silica.

Figure 4.23: The effect of reaction pH upon the surface area of nano-structured calcium silicate reinforced with different amounts of monomeric silica. 
Figure 4.24: The concentration of monomeric silica remaining in solution after reinforcement with varying amounts of monomeric silica and hydrochloric acid.

Figure 4.25: The concentration of calcium remaining in solution after reinforcement with varying amounts of monomeric silica and hydrochloric acid.

Figure 4.26: A scanning electron micrograph of nano-structured calcium silicate that has been reinforced to prevent collapse of the structure when removing water from within the pores upon drying at $110{ }^{\circ} \mathrm{C}$. .84

Figure 4.27: Alkaline darkening range of typical groundwood pulps over different $\mathrm{pH}$ values of the papermaking process when measured at the wetend, that is, before water removal from the pulp (Evans and Slozer, 2003)......85

Figure 4.28: Filler use in North America (Gill, 2005) . .86

Figure 4.29: Effect of lowering the $\mathrm{pH}$ of a nano-structured calcium silicate slurry upon its resulting composition.

Figure 4.30: The concentration of calcium in solution as a result of acidifying a nano-structured calcium silicate slurry..

Figure 4.31: The concentration of monomeric silica in solution as a result of acidifying a nano-structured calcium silicate slurry.

Figure 4.32: Effect of lowering the $\mathrm{pH}$ of a nano-structured calcium silicate slurry upon the \% solids.

Figure 4.33: Effect of lowering the $\mathrm{pH}$ upon the surface area and oil absorption capacity of nano-structured calcium silicate.

Figure 4.34: Scanning electron micrograph of base material at $\mathrm{pH} 12.0$ prior to the addition of acid.

Figure 4.35: Scanning electron micrograph of nano-structured calcium silicate at $\mathrm{pH} 10.0$.

Figure 4.36: Scanning electron micrograph of nano-structured calcium silicate at $\mathrm{pH} 7.0$

Figure 4.37: Powder X-ray diffractogram of nano-structured calcium silicate adjusted to $\mathrm{pH} 7.0$.

Figure 4.38: Thermal analysis curves of nano-structured calcium silicate with the $\mathrm{pH}$ altered post formation. 
Figure 4.39: Effect of lowering the $\mathrm{pH}$ of a nano-structured calcium silicate upon the whiteness and brightness of the material.

Figure 4.40: Effect of lowering the $\mathrm{pH}$ of nano-structured calcium silicate upon the colour of the material.

Figure 4.41: Development of the oil absorption capacity of 2-ethoxyethanol washed nano-structured calcium silicate formed using recycled filtrate.

Figure 4.42: The effect of lowering the pH of a slurry formed using recycled filtrate upon the surface area and oil absorption capacity.

Figure 4.43: Scanning electron micrograph of nano-structured calcium silicate formed with recycled filtrate and adjusted to $\mathrm{pH}$ 7.5. .98

Figure 5.1: Effect of variation in the $\mathrm{Ca} / \mathrm{Si}$ ratio during precipitation upon the physical properties after washing with 2EE.

Figure 5.2: The concentration of unreacted species in solution from reactions with varying $\mathrm{Ca} / \mathrm{Si}$ ratios.

Figure 5.3: Effect of reaction $\mathrm{pH}$ upon oil absorption capacity of material formed with $\mathrm{Ca} / \mathrm{Si}$ ratio of $1.1 \mathrm{using} \mathrm{pH}$ adjustment to maintain structure upon drying.

Figure 5.4: Effect of reaction $\mathrm{pH}$ upon oil absorption capacity of material formed with $\mathrm{Ca} / \mathrm{Si}$ ratio of 1.3 using $\mathrm{pH}$ adjustment to maintain structure upon drying.

Figure 5.5: Effect of reaction $\mathrm{pH}$ upon oil absorption capacity of material formed with $\mathrm{Ca} / \mathrm{Si}$ ratio of 0.9 using $\mathrm{pH}$ adjustment to maintain structure upon drying.

Figure 5.6: A photograph of slurries precipitated at different pHs after being allowed to settle. 108

Figure 5.7: Effect of reaction pH upon the oil absorption capacity and surface area of 2-ethoxyethanol treated samples.

Figure 5.8: Effect of $\mathrm{pH}$ upon the calculated total yield of precipitate formed

Figure 5.9: The oil absorption capacity of 2-ethoxyethanol treated samples produced with varying hydroxide and calcium ion concentrations. 113

Figure 5.10: The oil absorption capacity of untreated samples produced with varying hydroxide and calcium ion concentrations. 
Figure 5.11: The calculated total weight of material produced with varying hydroxide and calcium ion concentrations.

Figure 5.12: Effect of reaction temperature upon the development of oil absorption capacity of 2-ethoxyethanol treated material.

Figure 5.13: The effect of stirring throughout the ageing process upon the development of oil absorption capacity of 2-ethoxyethanol treated material.

Figure 5.14: The effect of concentration upon the maximum oil absorption achievable with a 2-ethoxyethanol treated material.

Figure 5.15: Schematic showing the general steps for the production of different types of nano-structured calcium silicates. 120

Figure 5.16: Photograph of the pilot plant for the production of nanostructured calcium silicate with feed tanks in the foreground.

Figure 5.17: Photograph of peristaltic pump, reaction vessel, and ageing tank of pilot plant for the production of nano-structured calcium silicate......

Figure 5.18: Photograph of the pilot plant in operation with the nanostructured calcium silicate slurry over flowing from the reaction vessel into the ageing tank.

Figure 5.19: Schematic showing the general steps for the production of different types of nano-structured calcium silicates.

Figure 5.20: The effectiveness of nano-structured calcium silicate for increasing the opacity of newsprint (Johnston et al., 2004)

Figure 5.21: The ability of nano-structured calcium silicate to reduce print through (Johnston et al., 2004).

Figure 6.1: Schematic of a hydrothermal resource.

Figure 6.2: The Taupo Volcanic Zone in the central North Island of New Zealand.

Figure 6.3: Schematic of a flash plant for the generation of electricity form geothermal water (Mink, 2005).

Figure 6.4: Schematic of a binary plant for the generation of electricity form geothermal water (Mink, 2005).

Figure 6.5: Source of geothermal water used in Kawerau

Figure 6.6: Work area beside weirbox at Kawerau. 
Figure 6.7: Transmission electron micrographs of K15 showing the presence of both calcium carbonate and nano-structured calcium silicate.

Figure 6.8: An X-ray diffractogram of K13 with sharp peaks due to the presence of calcium carbonate and calcium hydroxide.

Figure 6.9: Calculated amount of calcium carbonate present in materials precipitated from geothermal water at Kawerau. 144

Figure 6.10: Thermogravimetric analysis of sample K19. .145

Figure 6.11: Oil absorption capacities of materials precipitated from geothermal water at Kawerau

Figure 6.12: A scanning electron micrograph of K19 that shows a calcium carbonate crystal with relatively little porosity, amongst the typical porous structure of nano-structured calcium silicate.

Figure 6.13: Surface areas of materials precipitated from geothermal water at Kawerau.

Figure 6.14: Arsenic content of materials precipitated using geothermal water at Kawerau.

Figure 6.15: Relationship between the oil absorption capacities of materials precipitated using geothermal water at Kawerau and the arsenic content.... 149

Figure 6.16: The polymerisation curve for the formation of precipitated silica using geothermal water from the Kawerau field.

Figure 6.17: Polymerisation curve for the formation of precipitated silica using geothermal water from the Rotokawa field.

Figure 6.18: Oil absorption capacities of water washed materials precipitated from geothermal water at Kawerau from second series of experiments.

Figure 6.19: X-ray diffractogram of material prepared with the equivalent conditions of $\mathrm{K} 13$ (1000 mg/L Ca ${ }^{2+}$ and $20 \mathrm{~mL} \mathrm{25 \%} \mathrm{NaOH}$ ). 158

Figure 6.20: Oil absorption capacities of 2-ethoxyethanol washed materials precipitated from geothermal water at Kawerau from second series of experiments. 


\section{List of Tables}

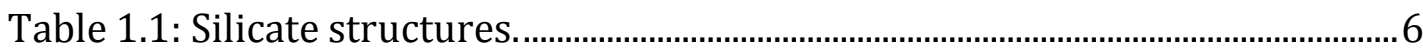

Table 1.2: Properties of commercial amorphous silica. (Waddell, 2006).............10

Table 1.3: Oil absorption of fillers (Wypych, 1999)...................................................... 20

Table 2.1: Composition details of sodium silicate solutions used. ............................ 23

Table 2.2: Major components of the calcium hydroxides used as measured by

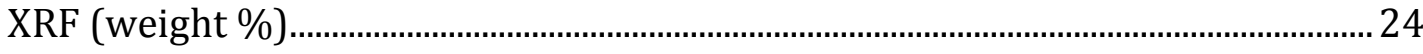

Table 2.3: Details of a typical 1 L reaction................................................................... 25

Table 2.4: Typical details for the reinforcement of a $1 \mathrm{~L}$ reaction............................30

Table 4.1: Surface tensions at $25^{\circ} \mathrm{C}$ of solvents used for washing samples (Lide, 2005)

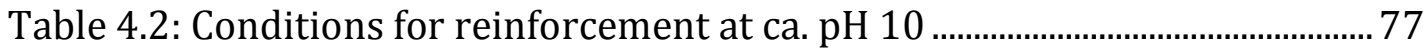

Table 4.3: Comparison of the increased weight of product with the weight of monomeric silica added. 80

Table 4.4: The pH of nano-structured calcium silicate slurries after reinforcement using different combinations of monomeric silica and hydrochloric acid. 80

Table 4.5: Oil absorption capacities of materials formed by continually recycling the filtrate $(\mathrm{g} / 100 \mathrm{~g})$ 99

Table 5.1: Calculation of the $\mathrm{Ca} / \mathrm{Si}$ ratio for nano-structured calcium silicate formed by varying the $\mathrm{Ca} / \mathrm{Si}$ ratio of reactants. 104

Table 5.2: Details of reactions performed to determine the effect of the reaction $\mathrm{pH}$ upon the oil absorption capacity. 105

Table 5.3: Details of reactions to determine the effect of $\mathrm{pH}$ upon the physical properties of 2-ethoxyethanol treated samples. 109

Table 5.4: The $\mathrm{pH}$ of each slurry produced following reaction with monomeric silica by varying the hydroxide and calcium ion concentrations.

Table 5.5: Typical properties of differently treated nano-structured calcium silicates prepared using conditions for optimising the oil absorption capacity. 
Table 5.6: A mass balance for the optimised preparation of nano-structured calcium silicate in untreated and modified forms.

Table 5.7: Concentration of major species in solution from the preparation of the base nano-structured calcium silicate slurry 122

Table 5.8: Properties of paper fillers (Hubbe, 2006) 125

Table 6.1: Existing and potential electricity generation of the Kawerau geothermal field (Hall, 2004).

Table 6.2: Details of reactions using geothermal water without the addition of sodium hydroxide during the first series of work at Kawerau.

Table 6.3: Details of reactions with the addition of sodium hydroxide for $\mathrm{pH}$ adjustment using geothermal water during the first series of work at Kawerau

Table 6.4: The proportion of minor components present for all samples prepared using geothermal water from the Kawerau field as determined by XRF spectroscopy. 141

Table 6.5: Composition details of samples precipitated from geothermal water from the first series of work at Kawerau.

Table 6.6: Details of reactions to form precipitated silica using geothermal water during the first series of work at Kawerau.

Table 6.7: Details of reactions using geothermal water from the Rotokawa field 152

Table 6.8: Composition of materials formed using Rotokawa geothermal water as determined using XRF 153

Table 6.9: The proportion of minor components present for all samples prepared using geothermal water from the Rotokawa field as determined by XRF spectroscopy. 153

Table 6.10: Details of reaction conditions for the precipitation of materials from geothermal water during the second series of work at Kawerau. 156 


\section{List of Abbreviations}

$\begin{array}{ll}\text { 2EE } & \text { 2-Ethoxyethanol } \\ \text { 2ME } & \text { 2-Methoxyethanol } \\ \text { AAS } & \text { Atomic absorption spectroscopy } \\ \text { AR } & \text { Analytical reagent } \\ \text { ASTM } & \text { American Society for Testing and Materials } \\ \text { a.u. } & \text { Arbitrary units } \\ \text { CIE } & \text { International Commission on Illumination } \\ \text { C-S-H } & \text { Calcium silicate hydrate } \\ \text { LOI } & \text { Loss on ignition } \\ \text { MAS } & \text { Magic angle spinning } \\ \text { NMR } & \text { Nuclear magnetic resonance } \\ \text { OA } & \text { Oil absorption } \\ \text { PDF } & \text { Powder diffraction file } \\ \text { ppm } & \text { Parts per million } \\ \text { rel. units } & \text { Relative units } \\ \text { SA } & \text { Surface area } \\ \text { SEM } & \text { Scanning electron microscopy } \\ \text { [SiO }]_{m} & \text { Monomeric silica concentration } \\ \text { TAPPI } & \text { Technical Association of the Pulp and Paper Industry } \\ \text { TEM } & \text { Transmission electron microscopy } \\ \text { TMS } & \text { Tetramethylsilane } \\ \text { w/w } & \text { Weight per weight } \\ \text { XRD } & \text { X-ray diffraction } \\ \text { XRF } & \text { X-ray fluorescence } \\ & \end{array}$




\section{Chapter 1}

\section{INTRODUCTION}

The discovery of a calcium silicate with a hitherto unseen nano-structure (Johnston et al., 2006) consisting of randomly stacked platelets (Figure 1.1) came about as a method to precipitate the monomeric silica in a geothermal fluid that had a dissolved silica concentration beneath the solubility of solid phase precipitated amorphous silica. Precipitation of silica from geothermal water super-saturated with dissolved silica concentrations above the solubility had until this time typically proceeded through a polymerisation method (Harper, 1994; Harper et al., 1997; Meyer, 1996) that produces a network structure (Figure 1.2). This method involved ageing the geothermal water to produce a sol of nano-sized silica particles and then using a relatively low concentration of calcium cations as a coagulant to precipitate the network structured amorphous silica. In order to precipitate the dissolved silica in geothermal water with a concentration beneath the silica solubility, a relatively high concentration of calcium was added under more highly alkaline

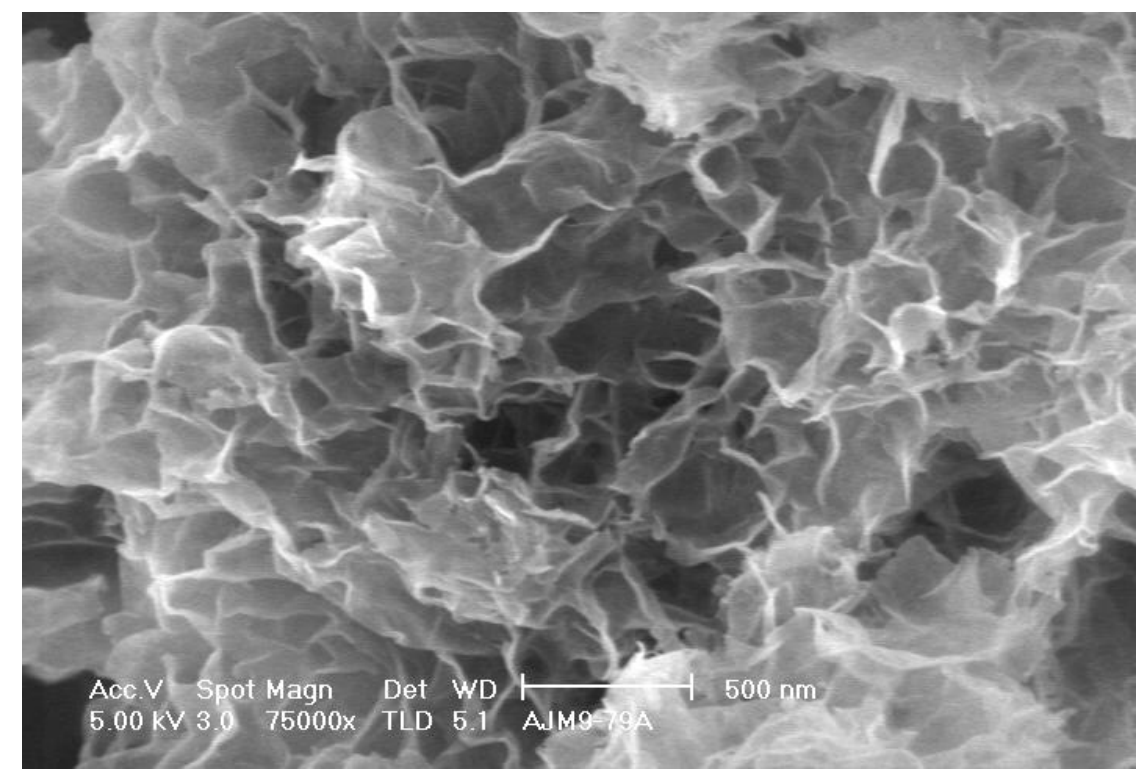

Figure 1.1: A scanning electron micrograph of the novel nano-structured calcium silicate revealing the porous nature of the material. 
conditions, which resulted in the formation of nano-structured calcium silicate. The material is described as a nano-structured calcium silicate to differentiate it from the numerous other calcium silicates and hydrated calcium silicates that exist with varying extents of atomic ordering. McGraw-Hill (2003) defines a nano-structure as "something that has a physical dimension smaller than 100 $\mathrm{nm}$, ranging from clusters of atoms to dimensional layers". This description was therefore chosen as the platelets that are responsible for the materials interesting physical properties have at least one dimension (thickness) within this range (and often all three dimensions are less than $100 \mathrm{~nm}$ ), when observed using scanning electron microscopy.

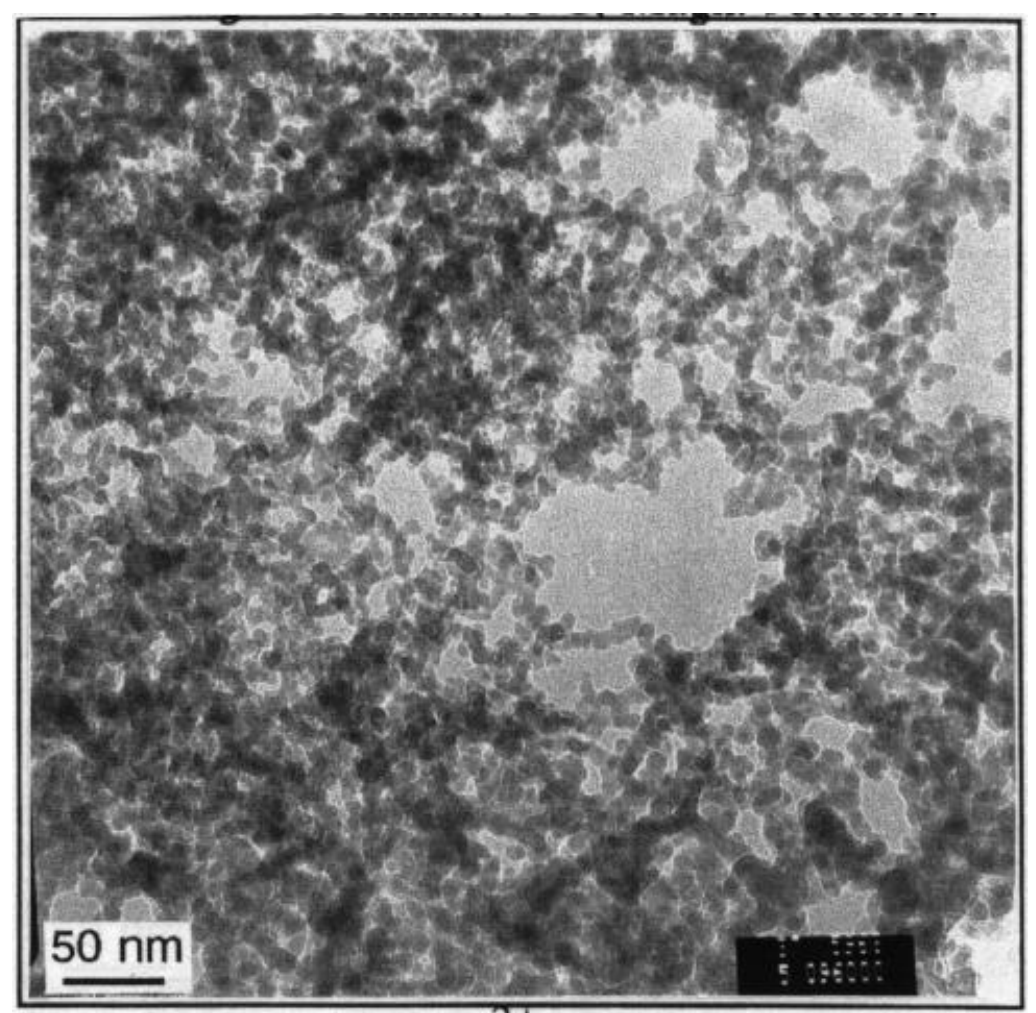

Figure 1.2: A transmission electron micrograph of a silica precipitated from geothermal water. (Meyer, 1996)

Nano-structured calcium silicate is formed as a result of the reaction between calcium ions and dissolved silica at an alkaline $\mathrm{pH}$ under controlled conditions of mixing, temperature and ageing time. The dissolved silica can be sourced from either geothermal water or sodium silicate, and is mainly present as $\mathrm{H}_{3} \mathrm{SiO}_{4}{ }^{-}$and $\mathrm{H}_{2} \mathrm{SiO}_{4}{ }^{2-}$ silicate ions at the highly alkaline conditions typically used of above pH 11 (Packter, 1986). The structure of the material is a result of a 
self-organisation over time and no templates or structure directing agents are used during the synthesis. A representation of the reaction is given below:

$$
\mathrm{H}_{3} \mathrm{SiO}_{4}^{-}+\mathrm{Ca}^{2+}+\mathrm{OH}^{-} \leftrightharpoons \mathrm{CaSiO}_{x}(\mathrm{OH})_{y}+\mathrm{H}_{2} \mathrm{O}
$$

Where: $\quad x$ is approximately $2-3$

$y$ is approximately $2-1$

The resulting poorly-ordered open framework structure of nano-sized platelets resembles a house of cards or gypsum "desert rose". It is this structure that provides the desirable physical properties of an accessible large pore volume and surface area that considerably exceeds those achieved from the network structure of precipitated silica particles. The structure consists of randomly shaped pores with varying pore sizes mainly in the macropore size range as they are greater than $50 \mathrm{~nm}$ (McNaught \& Wilkinson, 1997). These large pores enable to material to absorb a large quantity of oil, in excess of $600 \mathrm{~g}$ of oil/100 $\mathrm{g}$ of silicate, as well as provide ready access to the large surface area of up to $600 \mathrm{~m}^{2} / \mathrm{g}$. The typical fundamental particle size observed by SEM is ca. 1 to 5 $\mu \mathrm{m}$ with agglomerates up to $20 \mu \mathrm{m}$ observed by SEM and with laser scattering particle size analysis. X-ray diffractograms of nano-structured calcium silicates exhibit some broad peaks indicating that the material is very poorly crystalline and this lack of order at an atomic level probably contributes to the disorder observed in the structure on the macro- scale.

Given the widespread use of various silicas and silicates across many industries, the physical properties that this calcium silicate possesses suggest that there may be several possible applications for the material such as a liquid carrier, paper filler, or in a paper coating (Johnston et al., 2002). As well as the physical properties the material, the ability to perform chemistry on the surface also provides further potential applications. The ion-exchange capability can be used for the adsorption of metal ions (Cairns et al., 2006) and phosphate (Southam et al., 2004) from solution, the material can act as a catalyst support (Borrmann \& Johnston, 2002), while functionalising the surface could provide potential anti-corrosion or anti-bacterial applications (Johnston , 2007). 


\subsection{The $\left[\mathrm{SiO}_{4}\right]$ tetrahedral building block}

Silicon occurs in group IV A of the periodic table, and like all non-transition elements, tends to almost exclusively form four co-ordinate compounds with tetrahedral geometry. The most commonly observed example of silicon in this geometry is the orthosilicate anion $\left(\mathrm{SiO}_{4}^{4-}\right)$, which is the building block of all silicas and silicates. Due to silicon being significantly less electronegative than oxygen, the $\mathrm{Si}-\mathrm{O}$ bond is intermediate in character between ionic and covalent (Gibbs et al., 1994). Since Pauling (1929) put forward that the Si-O bond is approximately 50\% covalent and 50\% ionic in character, there has been an ongoing debate as to whether the bond is predominantly ionic or covalent (Pauling, 1980). Nevertheless, as ionic bonding is non-directional, the partially ionic character of the bond allows for more variation of bond angles for $\mathrm{Si}-\mathrm{O}-\mathrm{Si}$ siloxane linkages with a soft bending potential at the oxygen atom. When the calculated bending potential for $\mathrm{Si}-\mathrm{O}-\mathrm{Si}$ is compared with that of $\mathrm{Si}-\mathrm{S}-\mathrm{Si}$ (where the difference in electronegativity with the heteroatom is less), it has been found that not only is less energy required to distort the geometry from the energy minimum at the oxygen centre, but also the potential at the minimum is much softer (Figure 1.3).

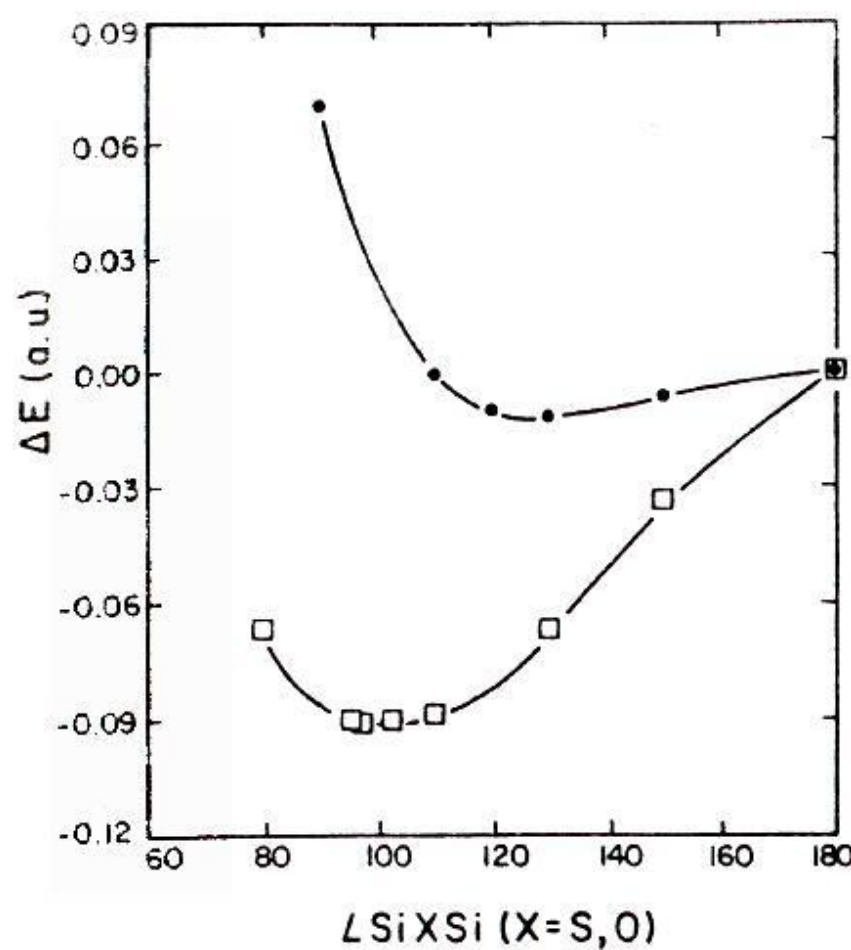

4
Figure 1.3: Calculated bending potentials for Si-O-Si (circles) and Si-S-Si (squares) units. (Burdett, 1988) 


\subsection{Silicates}

The flexibility of the Si-O-Si linkage, in combination with the different degrees of connectivity between the $\mathrm{SiO}_{4}^{4-}$ tetrahedral building blocks, leads to a wide variety of silicate structures that can be formed (Table 1.1). The connectivity of these building blocks can be described with " $Q$ " notation where a superscript defines the number of nearest neighbour silicon atoms attached to the tetrahedral corners (Fyfe, 1983; Falcone, 2000; MacKenzie \& Smith, 2002). Q ${ }^{0}$ represents discreet tetrahedra where the silicon atoms are not bound to any other silicon atoms over an oxygen bridge. $\mathrm{Q}^{1}$ represents where a tetrahedron shares only one apical oxygen with another tetrahedron, such as for either a dimer or the end-member of a chain. $Q^{2}$ represents tetrahedra that are arranged in chains or in discreet cyclic units, as each tetrahedron is bound to two other tetrahedra over oxygen bridges. $\mathrm{Q}^{3}$ connectivity is found in branched chains and sheets structures, while $Q^{4}$ is found in three-dimensional frameworks such as quartz. Silicate structures can accommodate a wide variety of cations to balance the charge of the fundamental structural unit, as well as possible substitution of silicon atoms in the structure (for example, by aluminium to form aluminosilicates). Pillared clays are silicates that find applications based upon their porosity due to cavities between the layers that make up the structure. These clays are able to selectively intercalate species as the interlayer spacing is uniform. However, the silicates most commonly used due to their porous structures are zeolites which are produced in millions of tons per annum.

\subsubsection{Zeolites}

Zeolites are crystalline silicate or aluminosilicate framework structures (Figure 1.4) with uniform channels in the micropore size range $(<2 \mathrm{~nm})$ of which more than 200 synthetic zeolite types (Valdes, 2006) and 50 natural zeolite minerals (Kuhl \& Kresge, 2000) are known to exist. The applications of zeolites range from water softening and purification using their ion exchange capability, to separation and removal of gases and solvents, through to being the most 
Table 1.1: Silicate structures.

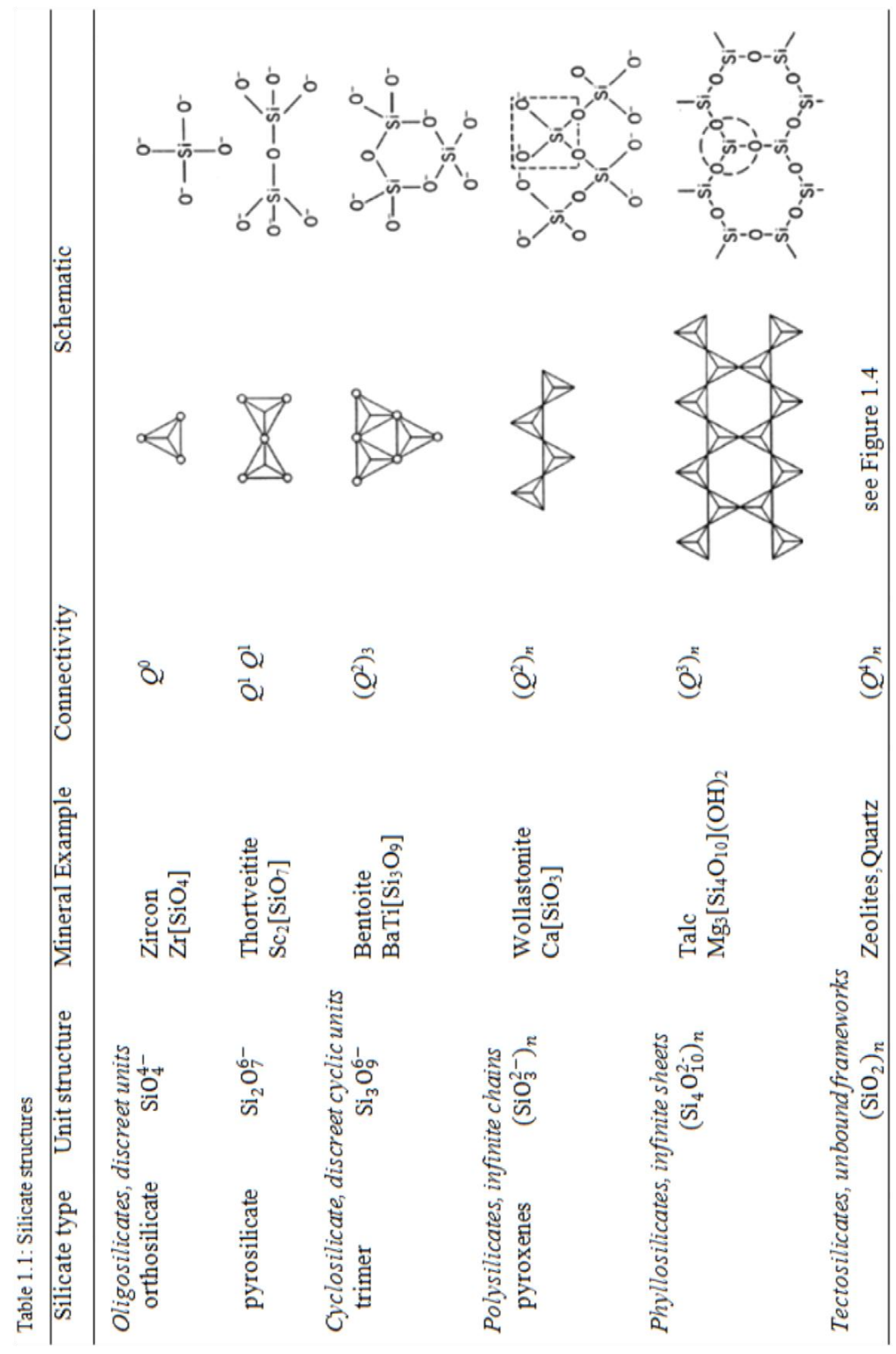


important catalysts in petrochemical refineries (Kuhl \& Kresge, 2000; Maple, 2006). Zeolite frameworks are constructed solely of $\left[\mathrm{SiO}_{4}\right]^{4-}$, or of $\left[\mathrm{SiO}_{4}\right]^{4-}$ and $\left[\mathrm{AlO}_{4}\right]^{5-}$ tetrahedra linked to one another through the oxygen atoms, although no direct link can occur between $\left[\mathrm{AlO}_{4}\right]^{5-}$ tetrahedra (Loewenstein rule). The charge imbalance in aluminosilicates, as a result of the substitution of aluminium for silicon, requires the inclusion of alkali metal and/or alkaline earth metal cations. The composition of aluminosilicate zeolites can be represented by $\mathrm{M}_{2 / m} \cdot \mathrm{Al}_{2} \mathrm{O}_{3} \cdot x \mathrm{SiO}_{2} \cdot y \mathrm{H}_{2} \mathrm{O}$ where $x \geq 2, \mathrm{M}$ is the charge balancing cation, $m$ is the cation valence, and $y$ is the amount water contained in the voids. The cations occupy the channels or interconnecting voids within the framework along with water molecules which can be removed reversibly, although under certain conditions can cause the collapse of the channel system (Flanigen, 2001). Cations that are commonly found in natural zeolites include sodium, potassium, magnesium, and calcium, while synthetic zeolites have been prepared with a wide range of cations from approximately one third of the periodic table (Kuhl \& Kresge, 2000).
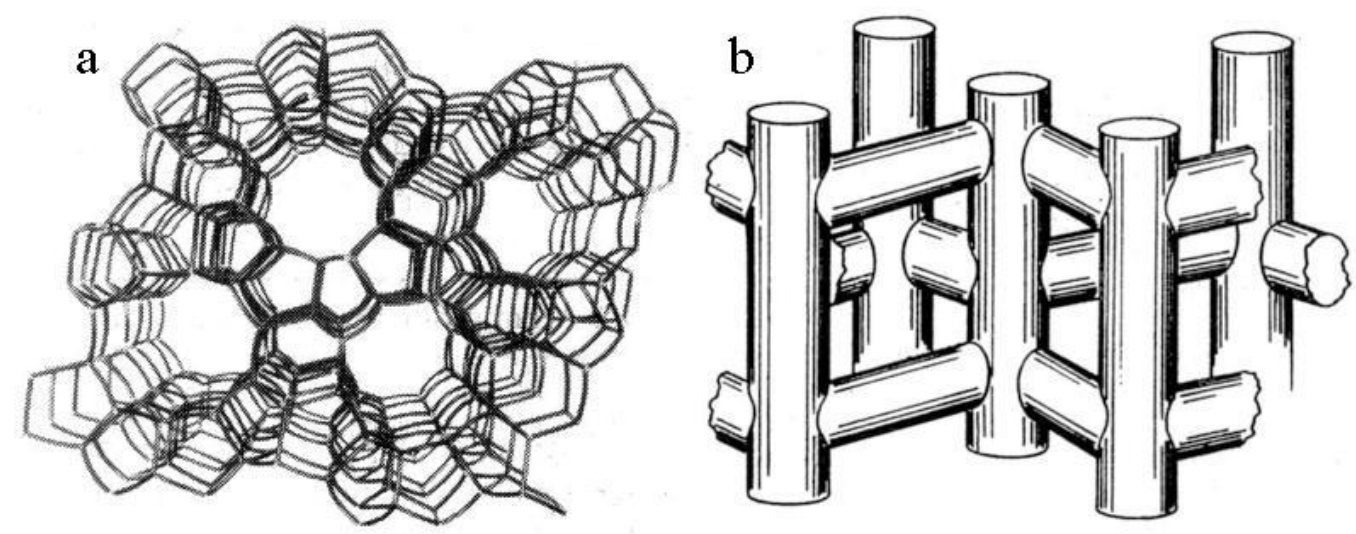

Figure 1.4: (a) Framework structure showing the topology of the molecular sieve ZSM-5 viewed in the direction of the main channel. (Flanigan et al., 1978) (b) A representation of the twodimensional channel system in ZSM-5. (Kokotailo et al., 1978)

The uniform pore size of zeolites leads to selectivity of what can be adsorbed based on the molecular size or shape, although polarity and activation energy are also important. Due to the narrow pores ( 0.3 to $1.3 \mathrm{~nm})$, zeolites possess high surface areas that in combination with their ability to accommodate a wide variety of cations makes them ideal for heterogeneous catalysis and adsorption applications such as for ion exchange and as sensors. ZSM-5 and its all-silica 
form silicalite have internal surface areas of ca. $350 \mathrm{~m}^{2} / \mathrm{g}$ although the total surface area can be as great as $550 \mathrm{~m}^{2} / \mathrm{g}$ when the external surface area of nanocrystals is taken into account (Song et al., 2004). The micropores and voids of zeolites may amount to $50 \%$ of the crystals by volume and range from 0.16 to $0.35 \mathrm{~cm}^{3} / \mathrm{g}$ (Flanigan, 2001). For heterogeneous catalysis and adsorption applications, the material will preferably have a two- or three-dimensional channel system for rapid intracrystalline diffusion, although the diffusion rate can still be a substantial hindrance to a process. In order to increase the diffusion rate through zeolites, focus has shifted to the development of hierarchical structures whereby macropores can provide easier access to the micropores. A macroporous form of silicalite templated around arrays of monodisperse polystyrene arrays has the much larger pore volume of 1.7 $\mathrm{cm}^{3} / \mathrm{g}$ compared with $0.2 \mathrm{~cm}^{3} / \mathrm{g}$ of the base material (Holland et al., 1999).

\subsubsection{Mesoporous molecular sieves}

Following the advancement of synthetic zeolites and the realisation of the limitations upon the size of molecules that could be adsorbed into their narrow pore sizes, the M41S family of mesoporous silicates and aluminosilicates was discovered by Mobil's scientists (Kresge et al., 1992). This family has three important members; MCM-41 with a one-dimensional hexagonal (P6mm) channel structure (Figure 1.5), MCM-48 a three-dimensional bicontinuous cubic structure $(I a 3 d)$, and MCM-50 a lamellar based mesostructure $\left(\mathrm{L}_{\alpha}\right)$.

Most uses of these materials are extensions of zeolite applications and they therefore tend to have pore sizes at the smaller end of the range in the definition of a mesoporous material (2 to $50 \mathrm{~nm}$ ). While the templating approach used in the synthesis of zeolites typically involves the crystallisation of a silicate around single molecules, the synthesis of M41S materials uses a group of molecules (i.e. micelles) as templating agents. By controlling the critical micelle concentration, it is possible to form cylindrical micelles with a hexagonal array around which the thin amorphous silicate or aluminosilicate walls can form. The solubilised silica cooperatively co-assembles with the surfactant to form liquid crystal phases during the synthesis. The organic 
structure-directing agents, such as alkyltrimethylammonium halides or triblock copolymers, not only template the synthesis of highly ordered mesopores but also allow the pore size to be tailored due to the direct relationship that exists between the alkyl chain length of the surfactant template molecule and the pore size of the final product.

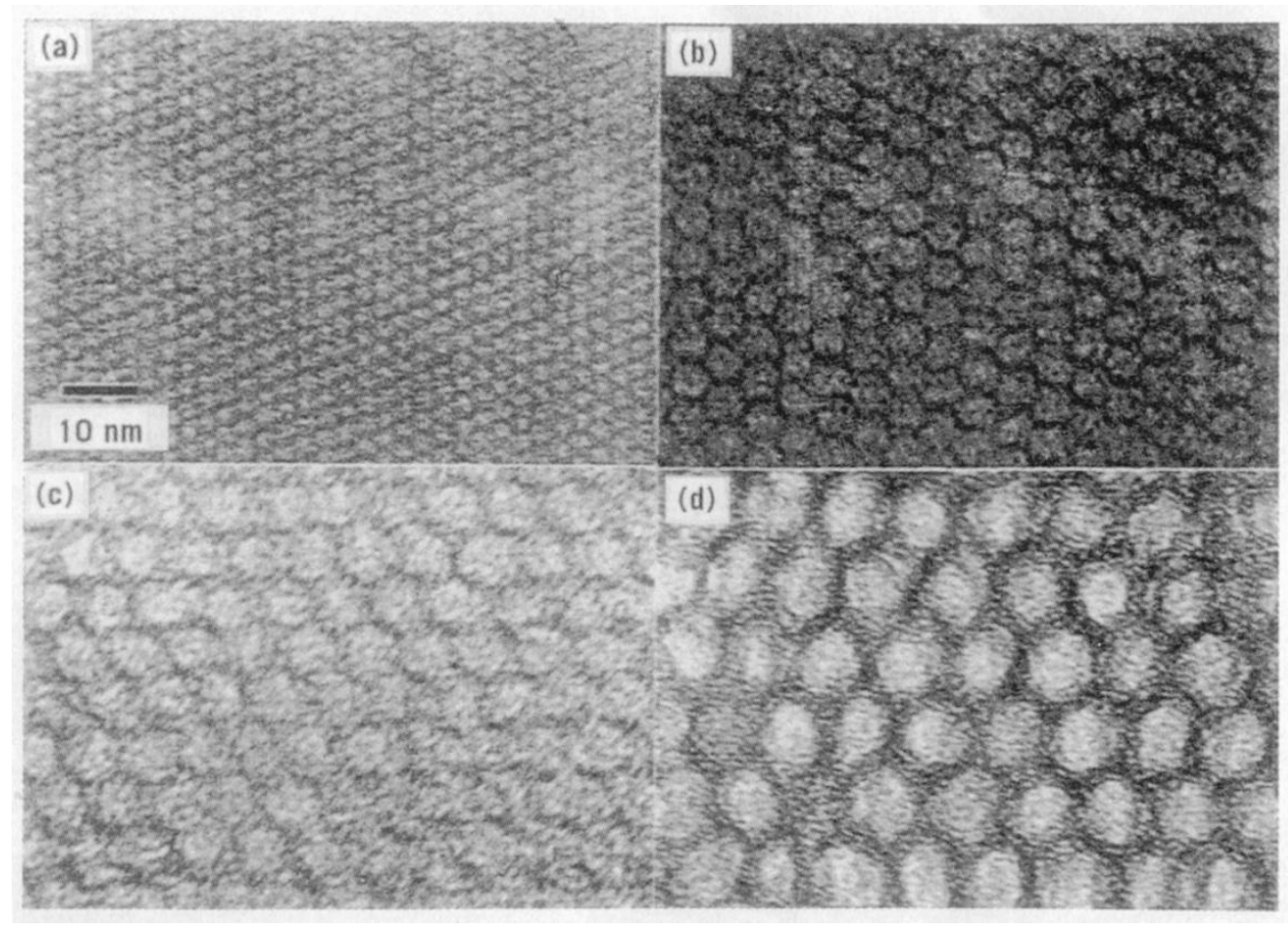

Figure 1.5: Transmission electron micrographs of MCM-41 having hexagonal pores in a regular honeycomb arrangement with diameters of (a) $2 \mathrm{~nm}$, (b) $4 \mathrm{~nm}$, (c) $6.5 \mathrm{~nm}$, and (d) $10 \mathrm{~nm}$ (Beck et al., 1992).

Like aluminosilicate zeolites, mesoporous molecular sieves that contain aluminium need cations for charge neutralisation, which leads to the possibility of selecting a species based on potential catalytic behaviour. Due to the narrow pore walls mesoporous molecular sieves typically exhibit very high surface areas in the order of $1000 \mathrm{~m}^{2} / \mathrm{g}$ (Flanigan, 2001). The larger pore diameters also result in higher pore volumes (ca. $1.5 \mathrm{~cm}^{3} / \mathrm{g}$ ) than those of zeolites, while also improving diffusion through the material. Due to the walls of mesoporous molecular sieves being amorphous, these materials are typically a lot less stable than zeolites at the elevated temperatures catalytic applications require. 


\subsection{Silicas}

Silicas are used in many applications due to the wide range of physical properties they can possess as materials with high surface areas or large pore volumes, in combination with their chemical properties whereby the surfaces can be modified or rendered relatively chemically stable. Synthetic amorphous silicas generally fall into four categories; pyrogenic silica (also known as fumed silica) which is made at a high temperature, and silica sols (colloidal silica), silica gels, and precipitated silicas that are all produced in a wet process. Each type of silica possesses unique properties due to either the structure of the material or the method of manufacture (Table 1.2).

Table 1.2: Properties of commercial amorphous silica. (Waddell, 2006).

\begin{tabular}{lcccc}
\hline Property & $\begin{array}{l}\text { Colloidal } \\
\text { silica }\end{array}$ & $\begin{array}{l}\text { Silica gels } \\
\text { (dry) }\end{array}$ & $\begin{array}{l}\text { Precipitated } \\
\text { silica }\end{array}$ & $\begin{array}{c}\text { Pyrogenic } \\
\text { silica }\end{array}$ \\
\hline $\mathrm{SiO}_{2}, \%$ & $15-50$ & $96.5-99.6$ & $85-95$ & $98.3-99.8$ \\
Surface area, $\mathrm{m}^{2} / \mathrm{g}$ & $50-750$ & $200-800$ & $25-700$ & $35-410$ \\
Oil absorption, g/g & $0.9-3$ & 1.5 & $1.5-3.5$ & $0.5-3$ \\
Weight loss, \% & & & & \\
$\quad$ at $105{ }^{\circ} \mathrm{C}$ & $50-80$ & & $4-7$ & $0.5-2.5$ \\
at $1200{ }^{\circ} \mathrm{C}$ & $50-90$ & $2-17.5$ & $10-14$ & $1-4$ \\
Density, g/cm ${ }^{3}$ & $2.2-2.3$ & 2.22 & $1.9-2.1$ & 2.16 \\
Bulk density, g/cm ${ }^{3}$ & $1.2-1.4$ & $0.1-0.9$ & $0.03-3$ & $0.03-0.12$ \\
Particle size & & & & \\
$\quad$ ultimate, $\mathrm{nm}$ & $4-60$ & $1-100$ & $5-50$ & $5-50$ \\
$\quad$ aggregate, $\mathrm{nm}$ & & & $100-500$ & $100-1000$ \\
$\quad$ agglomerate, $\mu \mathrm{m}$ & & $3-25$ & $1-50$ & $1-3$ \\
Refractive index, $n_{\mathrm{D}}$ & $1.35-1.45$ & $1.35-1.45$ & 1.45 & 1.45 \\
\hline
\end{tabular}

Amorphous silicas are not truly amorphous due to the presence of regions of atomic order, or crystals of extremely small size and are therefore sometimes also referred to as micro-amorphous silicas. These materials do however only give a single broad band using standard X-ray diffraction methods and therefore are also sometimes described as being X-ray amorphous. Commercial manufacture of silicas via a wet process typically use a soluble silicate as the source of silica, the most commonly used being sodium silicate which is produced over a wide range of stoichiometric and non-stoichiometric 
compositions. Depending upon the metasilicate ratio $\left(\mathrm{SiO}_{2} / \mathrm{M}_{2} \mathrm{O}\right.$, where $\mathrm{M}$ can be $\mathrm{Na}, \mathrm{K}$, or $\mathrm{Li}$ ), the solids concentration, and the $\mathrm{pH}$, solutions of silicates can contain differing proportions of monomeric silicate ions, dimeric silicate ions, or polymeric silicate ion micelles (Weldes \& Lange, 1969; Falcone, 2000). This is due to the completeness of the network breakdown of the precursor glass during the manufacture of the soluble silicate. A liquid alkoxide precursor such as $\mathrm{Si}(\mathrm{OR})_{4}$ (where $\mathrm{R}=\mathrm{CH}_{3}, \mathrm{C}_{2} \mathrm{H}_{5}$, or $\mathrm{C}_{3} \mathrm{H}_{7}$ ) can also be used as a source of monosilicic acid by hydrolysis with water. In industry, sodium silicates are much more commonly used due to their lower cost, but they do tend to form silicas with more impurities (including elements such as $\mathrm{Na}, \mathrm{Al}, \mathrm{Ca}, \mathrm{Fe}$ ) than those produced using alkoxysilanes.

\subsubsection{Colloidal silica}

All of the silicas manufactured via the wet process undergo the same initial condensation polymerisation process through to the formation of silica sol particles. The polymerisation of silica takes place when the concentration of monosilicic acid (monomeric silica) in solution exceeds the solubility of amorphous silica (ca. 100-200 mg/L at ambient temperature and neutral pH) and there is no solid phase present on which the silica can be deposited. These conditions are met when an alkaline soluble silicate solution (typically $\mathrm{pH}>11$ ) that contains a high concentration of dissolved silica is acidified, due to the effect that $\mathrm{pH}$ has upon solubility (Figure 1.6). 


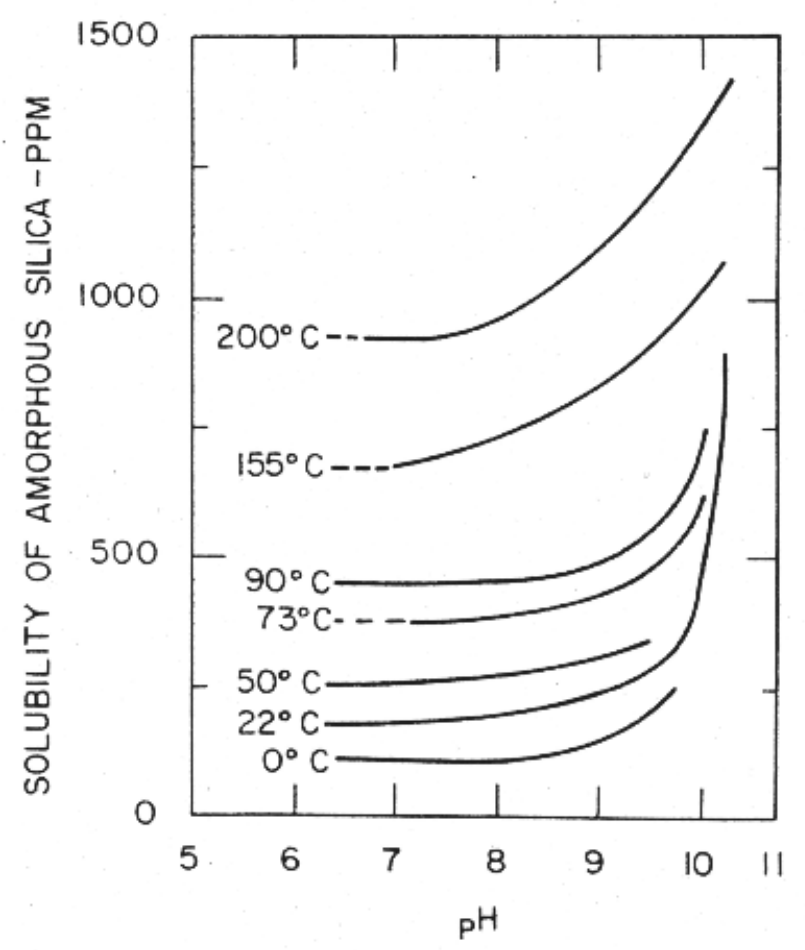

Figure 1.6: Solubility of amorphous silica at different temperatures over a pH range (Iler, 1979).

The rate of polymerisation is proportional to the hydroxide concentration above $\mathrm{pH} 2$, although above $\mathrm{pH} 10$ the rate constant of depolymerisation $\left(k_{-2}\right)$ becomes significant. The polymerisation occurs most readily when the $\mathrm{pH}$ of the solution is equal to the $\mathrm{p} K_{a}$ of the participating silanol group, which for monosilicic acid is 9.9 (Lide, 2005).<smiles>O[Si](O)(O)O[CH+]O[Si](O)(O)O</smiles>

The initial formation of a dimer is then followed by subsequent reaction of the polymerisation product with more monosilicic acid leading to the formation of cyclic structures due to the thermodynamic preference of siloxane (-Si-O-Si-) chains over silanol (-Si-OH) groups. These cyclic structures are then extended by addition of further monomers and are linked to other rings resulting in the formation of a three-dimensional network of randomly cross-linked polymer chains (Figure 1.7). These molecules then condense internally, thereby 
maximising siloxane linkages, to form small primary particles approximately 1 to $3 \mathrm{~nm}$ in diameter with external silanol groups.

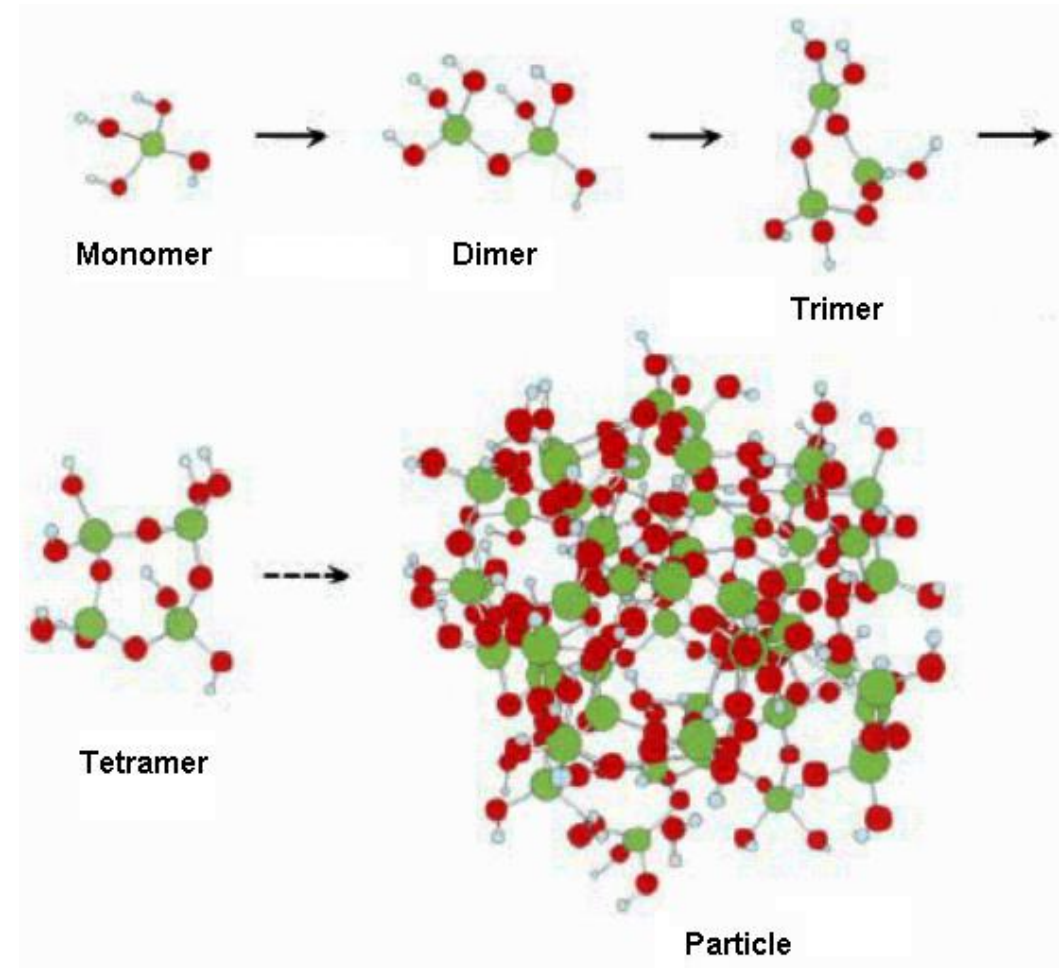

Figure 1.7: A representation of the growth of a sol particle from the start of polymerisation: green, silicon; red, oxygen; and white, hydrogen. (Coradin \& Lopez, 2003)

Above pH 7 in the absence of salts, the ionisation of surface silanol groups provides a sufficient negative charge on the surfaces to cause repulsion between the sol particles and therefore causes the formation of a stable colloid. Due to smaller particles being more soluble than larger ones, the larger particles grow at the expense of the smaller particles, therefore increasing the size of the particles whilst reducing the number of particles. This Ostwald ripening process leads to a sol of uniformly sized particles that can grow up to ca. $100 \mathrm{~nm}$ in diameter. The process of polymerisation can be shown graphically with the reduction of monosilicic acid concentration over time (Figure 1.8). The polymerisation curve has an initial induction period that is followed by the decrease in concentration of monomeric silica. The shape of the polymerisation curve can vary greatly due to different conditions. For example, at a $\mathrm{pH}>7$ the ionisation of polymer species leads to rapid polymerisation of monomers, which at higher temperatures can lead to the induction period not 
being observed experimentally. During the induction period the monomeric silica concentration decreases slightly as the particles nucleate. The rapid decrease in concentration of monomeric silica during secondary particle growth is coupled with an increase in the number of sol particles and growth of the particles as the polymerisation process continues. The curve finally levels out at the equilibrium solubility of the silica under the experimental temperature and $\mathrm{pH}$ conditions.

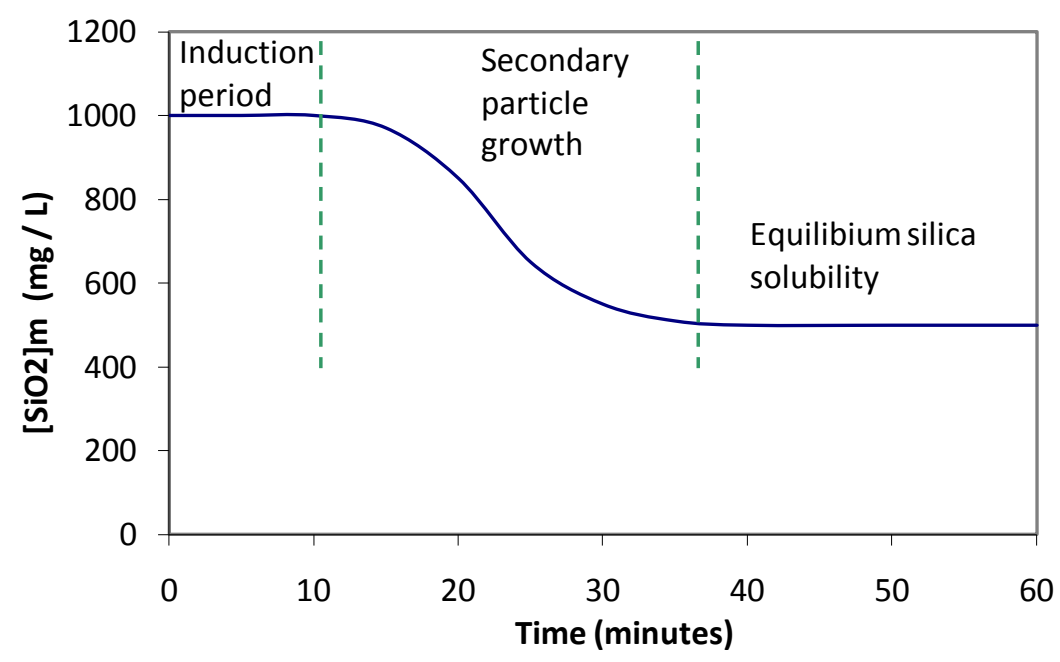

Figure 1.8: A general polymerisation curve showing the concentration of monomeric silica against time.

\subsubsection{Silica gels}

Below pH 7 there is not sufficient charge on the sol particles for electrostatic repulsion to keep them apart, therefore allowing for siloxane linkages to form between surface silanol groups when particles collide. The linkages created between the sol particles eventually lead to the formation of a threedimensional gel network (Figure 1.9). The formation of silica gels also occurs above $\mathrm{pH} 7$ in the presence of salts due to the neutralisation of the surface charge on the particles by the cations in solution. 


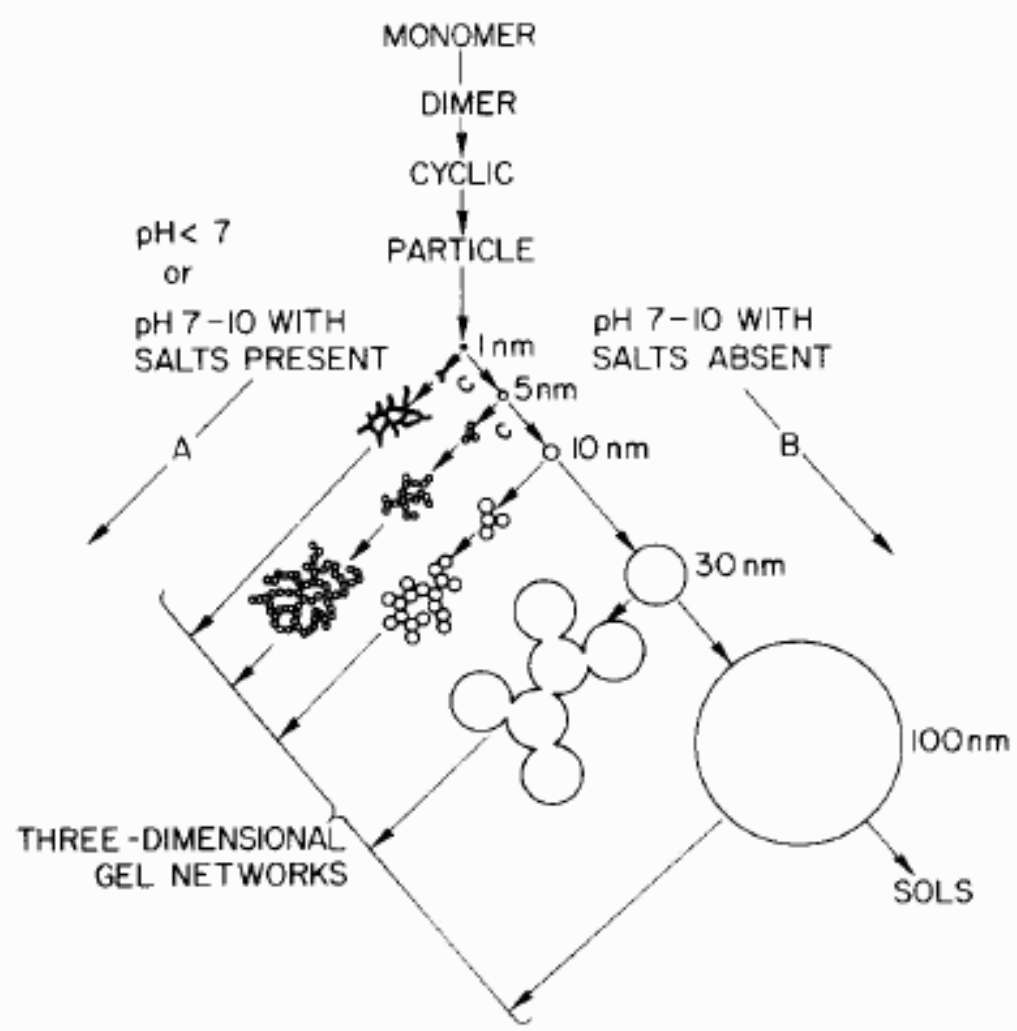

Figure 1.9: The polymerisation of silica with the formation of networks (A) or the growth of sol particles (B). (Iler, 1979)

Once the gel has been formed there are two main types of dried silica that can be formed. Xerogels are formed by removing the liquid medium (typically water) from a gel which causes the structure to be compressed and porosity decreased by some degree as a result of surface tension forces due to hydrogen bonding during liquid removal. In order to form aerogels, the liquid is removed in a way to prevent any collapse or change in the structure during liquid removal such as by using critical point drying. As aerogels maintain their pore structure when the pore liquid of the precursor gel is replaced with air, the resulting silicas are highly porous (80 to $99.8 \%$ porosity) with extremely low densities (0.003 to $0.5 \mathrm{~g} / \mathrm{cm}^{3}$ bulk density).

\subsubsection{Precipitated silicas}

The stable sol that exists due to mutual repulsion in alkaline conditions with salts absent can also be destabilised by the addition of a cationic agent which acts as a coagulant to form loose aggregates that are known as precipitated 
silicas. The destabilisation is due to bonding between the sol particles and the cationic agent and results in a bridge between the particles (Figure 1.10).

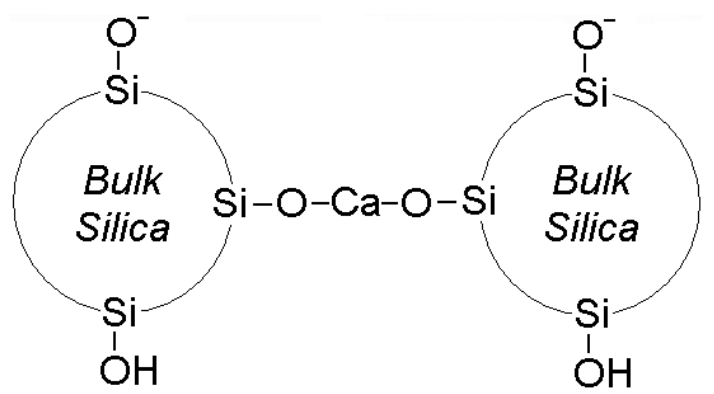

Figure 1.10: A representation of the bridge formed by a cationic agent (calcium in this case) due to the cation bonding to some of the ionised silanol groups.

The bonding between the ultimate colloidal particles is weaker than that formed by the siloxane linkages during the formation of a gel and can be easily broken by mechanical means. As a result of this, separate discreet particles of silica are formed with each consisting of a three-dimensional network (Figure 1.11).

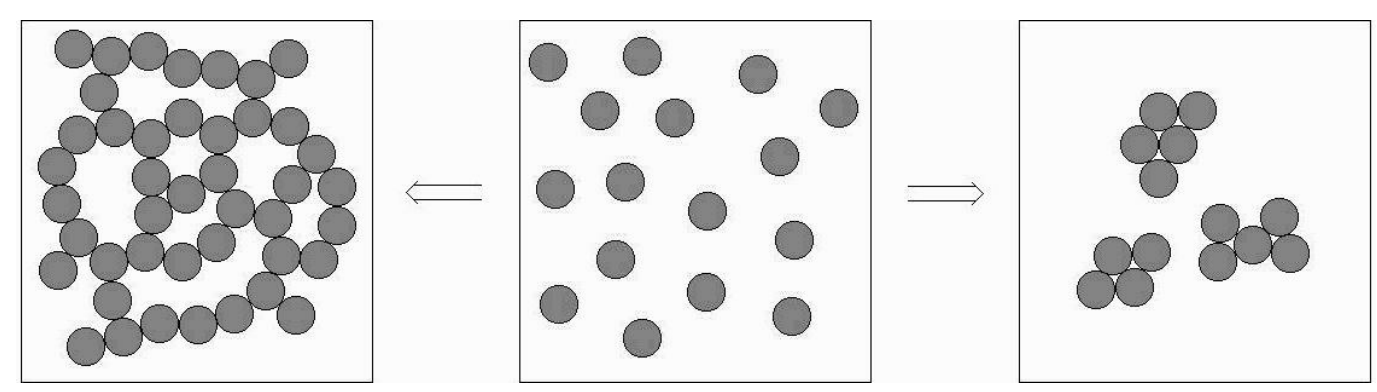

Figure 1.11: Representations of a gel network (left) and discreet precipitated silica particles (right) formed from the sol particles of a stable silica colloid (centre).

\subsubsection{Pyrogenic silicas}

Pyrogenic silicas are recovered from the vapour-phase as extremely finely divided powders. The most common method of production is the vapour-phase hydrolysis (ca. $1000{ }^{\circ} \mathrm{C}$ ) of silicon tetrachloride, although other methods include the vaporisation of silica, vaporisation and oxidation of silicon, and high temperature oxidation and hydrolysis of silicon compounds such as silicon esters. Due to the absence of liquid water during formation, pyrogenic silicas have less hydration on the surface compared with other amorphous silicas and because of this are used in rubber applications where low levels of surface water per unit surface area are required. 


\subsubsection{Biogenic silicas}

Nature creates a wide variety of fascinating amorphous biogenic silica structures due to a high level of control during the formation. This control is exhibited despite the silica source being available in low concentrations (below saturation concentration) and reaction conditions being mild with a near neutral $\mathrm{pH}$ and ambient temperatures. Two examples of the level of complexity achieved are sponges (which can have a silica content from 1 to $90 \%$ ) that form spicules made of concentric layers of silica hydrate that allow anchoring of the sponge to the sea floor, and unicellular diatom algae that deposit silica as an external skeleton with a structure similar to that of MCM-41 (Figure 1.12).
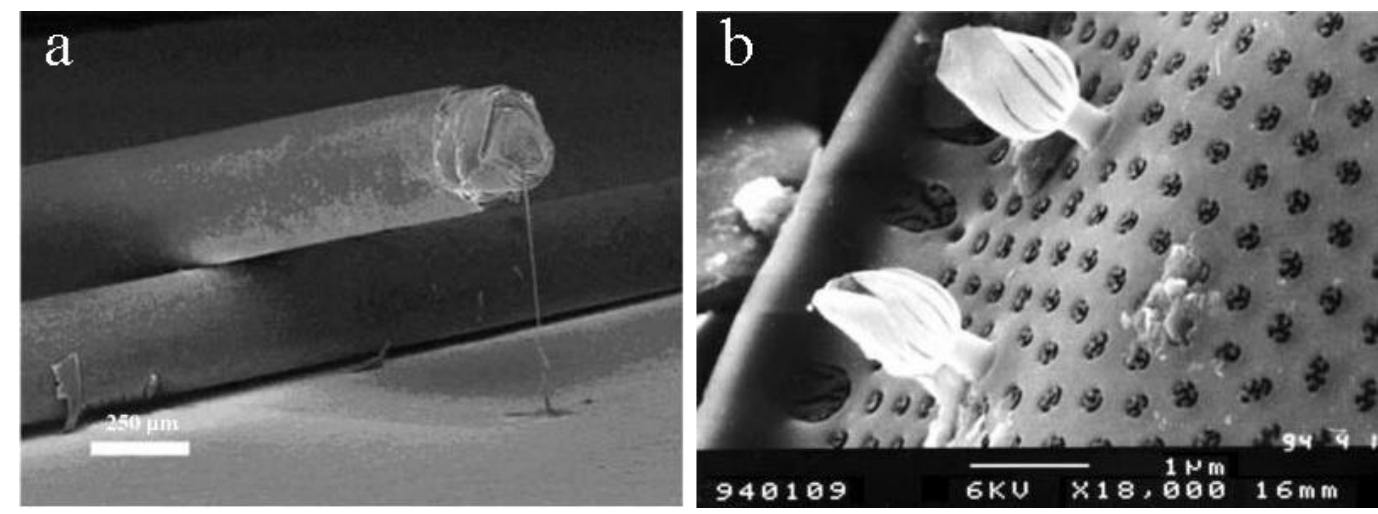

Figure 1.12: Scanning electron micrographs of biogenic silicas. (a) The concentric layers of a sponge spicule. (b) The pores in the shell of a diatom (Thalassiosira sp. frustule). (Coradin \& Lopez, 2003).

\subsection{Macroporous materials}

An advantage materials with pore-sizes in the macropore size range of $>50 \mathrm{~nm}$ have over materials with a smaller pore-size is that they have less diffusional resistance. This allows easier mass transport through the structure, as well as the ability to accommodate larger guest molecules such as biological compounds or polymers. Three-dimensional macroporous materials with long range order can be prepared with the use of templating agents much like zeolites and mesoporous molecular sieves. One method to produce these larger pore-size materials uses colloidal crystals consisting of ordered arrays of polymer or silica microspheres as templates that can be removed by thermal or 
chemical methods (Velev et al., 1997). The colloidal crystals can be formed by gravitational settling, centrifugation, convective assembly, or electrophoretic deposition. The voids between the templates can be filled with a wide variety of metal oxides prepared from precursors using sol-gel or salt precipitation methodologies (Figure 1.13).
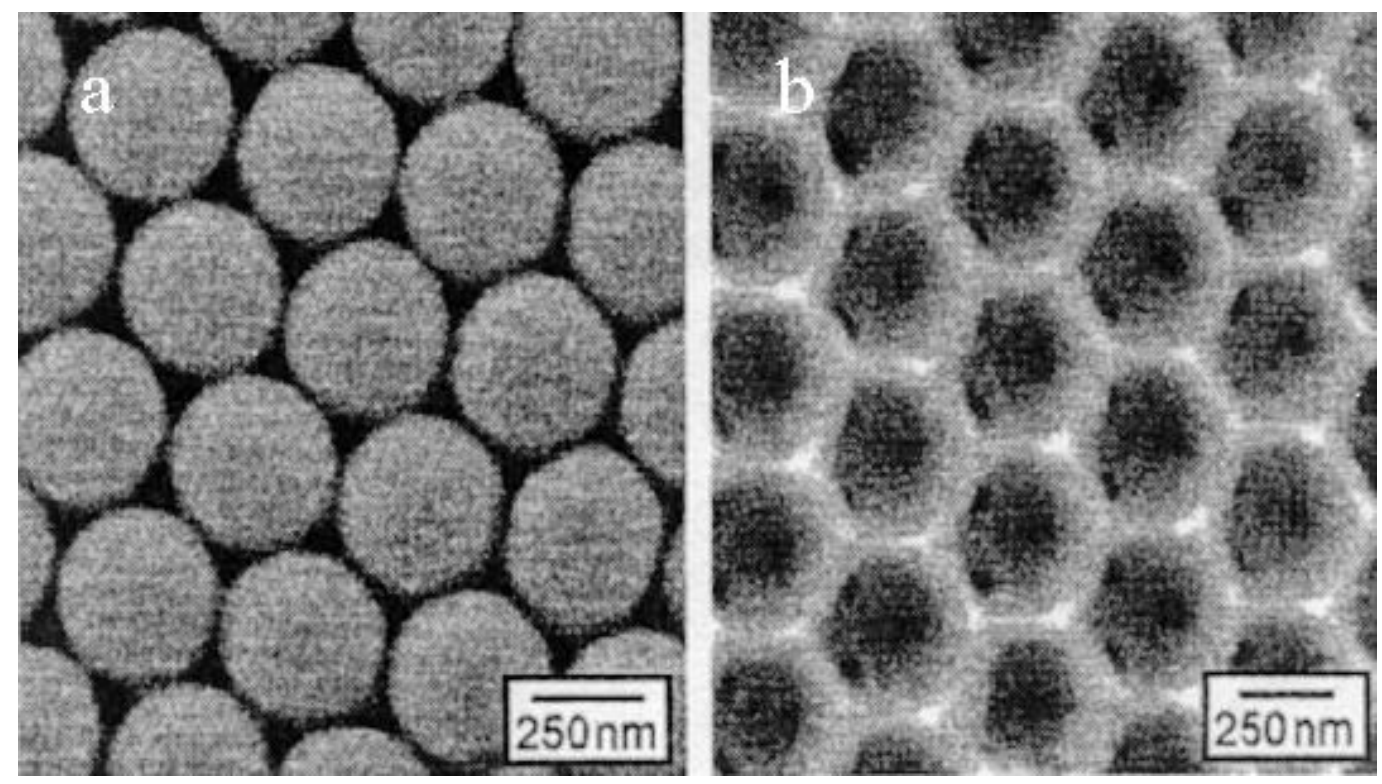

Figure 1.13: Scanning electron micrographs of mesoporous silica and its template. (a) The colloidal crystal array of monodisperse poly (methyl 2-methylpropenoate) spheres that act as the template, with a face-centred cubic structure reminiscent of opals. (b) The "inverse-opal" structure of a three-dimensionlly ordered macroporous silica after the removal of the template, with dark windows between pores due to contact points of the templating spheres. (Schroden \& Stein, 2004)

When the voids are filled with zeolites or mesoporous silica a hierarchical structure is formed that contains both macropores and either micro- or mesopores. Valtchev (2002) used slow evaporation of the solvent to fill the voids with silicalite-1 nano-particles, while Li et al. (2007) formed mesoporous silica in the voids (Figure 1.14). These hierarchical materials improve the diffusion to the narrower channels of the structure to improve their performance in applications such as heterogeneous catalysis, sensing, and separation. Another method to produce similarly ordered macroporous materials is to also use a sol-gel process whereby an inorganic material, such as a metal oxide, is deposited around the exterior of templating droplets in a monodisperse emulsion (Imhof \& Pine, 1997). Like zeolites and mesoporous molecular sieves, the narrow pore-size range of these ordered macroporous structures formed by the use of templating agents allows selectivity of guest 
molecules, although most interest in these materials is focussed on their application as a photonic band gap material in the field of optics.

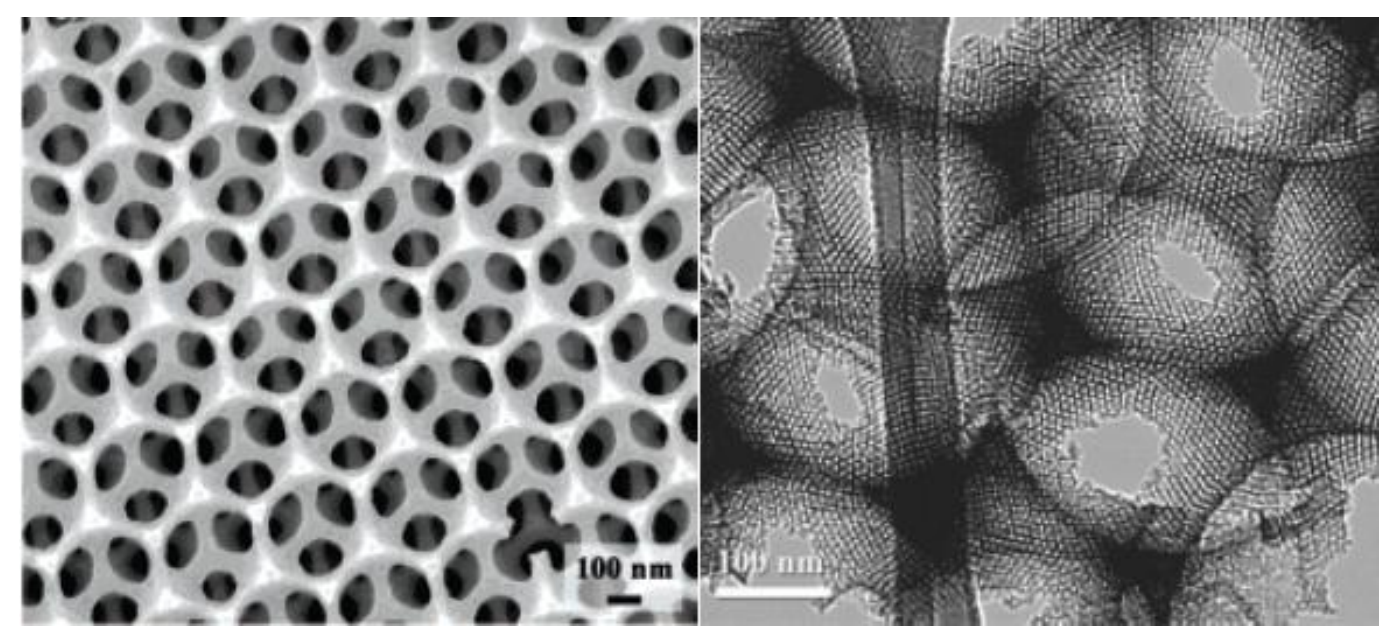

Figure 1.14: A hierarchical material with the walls of the macropores (left) made of mesoporous silica (right) (Li et al., 2007).

Examples of macroporous materials with no long range order include the synthesis of cellular foams (Figure 1.15a) formed by Freon bubbles dispersed in a sol of a metal oxide and stabilised by the increasing viscosity due to gel formation (Wu et al., 1990). An aragonite polymorph of calcium carbonate with a three-dimensional structure (Figure 1.15b) resembling that of nanostructured calcium silicate was formed by oil-water-surfactant bicontinuous microemulsions supersaturated with calcium bicarbonate (Walsh \& Mann, 1995). It is proposed that the self-organised foam of oil droplets act as a structural template around which the rapid mineralisation occurs.
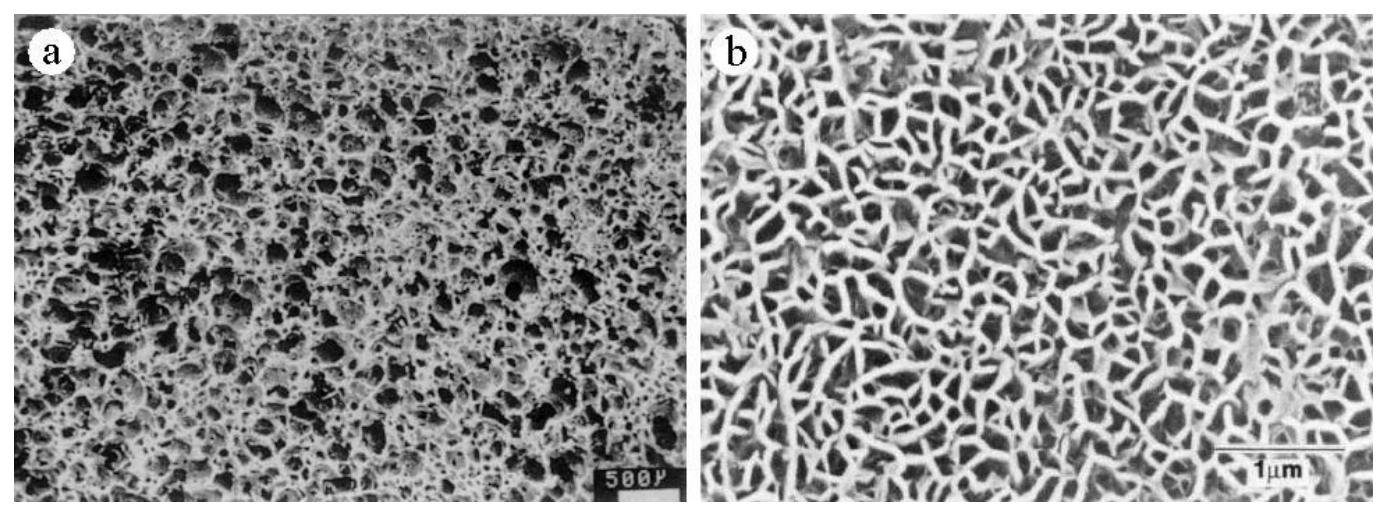

Figure 1.15: Scanning electron micrographs of macroporous materials with no long range order. (a) An inorganic cellular foam. (b) A cellular aragonite framework on a metal substrate. 


\subsection{Scope of thesis}

The unique structure of nano-structured calcium silicate provides a material that has different physical and chemical properties than other porous materials and therefore the potential to perform more favourably in certain applications. One of the most significant properties of the material is the very large pore volume and consequently large liquid absorption capacity due to the macropores in the framework, as observed with scanning electron microscopy. Nano-structured calcium silicates with oil absorption capacities in excess of $400 \mathrm{~g} / 100 \mathrm{~g}$ (reinforced) and $600 \mathrm{~g} / 100 \mathrm{~g}$ (2-ethoxyethanol treated) have been prepared. These values compare favourably with other materials that are used as fillers for a variety of applications, particularly silicas that are often employed for their porous nature (Table 1.3).

Table 1.3: Oil absorption of fillers (Wypych, 1999).

Oil absorption Filler (the range of a particular filler group is given in range parentheses)

(g/100 g)

below 10

barium sulphate (8-28), barium \& strontium sulfates (9.511.5)

10-19.9 aluminium trihydroxide (12-41), calcium carbonate (13$21)$, ferrites (10.8-14.8), glass beads (17-20), iron oxide (10-35), fused silica (17-27), quartz, tripoli (17-20), sand (14-28), titanium dioxide (10-45), wollastonite (19-47), zinc sulfide (13-14)

20-29.9 aluminium oxide (25-225), cristobalite (21-28), feldspar (22-30), kaolin (27-48), slate flour (22-32), talc (22-57)

30-49.9 barium metaborate (30), ball clay (36-40), bentonite (36$52)$, carbon black (44-300), magnesium hydroxide (40-50)

50-100 attapulgite (60-120), graphite (75-175), kaolin beneficiated (50-60), kaolin calcinated (50-120), mica (65$72)$, precipitated silica (60-320), silica gel (80-280), wood flour (55-60)

over 100 cellulose fibre (300-1000), diatomaceous earth (105-190), fumed silica (100-330), hydrous calcium silicate (290), perlite (210-240) 
The large pores of nano-structured calcium silicate allow ready access to a large surface area (over $500 \mathrm{~m}^{2} / \mathrm{g}$ when 2-ethoxyethanol washed and $300 \mathrm{~m}^{2} / \mathrm{g}$ for reinforced) which can to be functionalised through a surface chemistry that is only just beginning to be explored. A three-dimensional channel system greatly improves diffusion through the structures of zeolites when compared with twodimensional systems (Li et al., 2001) and therefore good diffusion could be expected for nano-structured calcium silicate.

Preparation of the nano-structured calcium silicate is very simple compared with the other materials previously discussed. The reaction is carried out at room temperature and at atmospheric pressure unlike the formation of zeolites or mesoporous molecular sieves that both use hydrothermal syntheses. Development of the structure also does not involve any structure directing agents or templates, unlike other macroporous materials, zeolites, and mesoporous molecular sieves. While this is an advantage in terms of the simplicity of the reaction, it does result in a material that does not have a tuneable narrow pore size range and is therefore not suitable for applications where size exclusion is important. However, the bulk physical properties of oil absorption capacity and surface area are able to be specifically designed through control of the precipitation process and the use of post-formation treatments upon the material. Due to the overall simplicity of the process it is anticipated that there should not be insurmountable issues in potentially scaling-up of the method of production to meet potential commercial demands. This work also focuses upon how aspects of the preparation of nano-structured calcium silicate will affect possible production on a large scale. The relative simplicity of the process in combination with the use of common precursor materials that are readily available in bulk quantities present the opportunity to produce a highly useful material inexpensively and therefore be potentially applied in everyday commodities.

The aim of this thesis is to increase the understanding and applicability of nano-structured calcium silicate. This objective will be met by transferring the process for preparing the material from using geothermal water with a low 
dissolved silica content to the use of sodium silicate as a precursor and therefore produce the material at much higher concentrations. The production of the nano-structured calcium silicate using geothermal water is also explored, although this system is more complex and by its very nature has limits as to where it could be applied. The formation of the material is studied using the less complex reaction conditions with sodium silicate as the precursor so that the process may be more clearly understood. Due to the highly disordered nature of the material, little useful information can be gained at an atomic level, therefore the bulk properties of the material are studied in greater depth with particular attention paid to the maximisation of the properties that may be advantageous for applications of porosity (oil absorption capacity) and surface area. The optimisation of these properties will come about as a result of varying reaction conditions and measuring what effects they have. The modification of nano-structured calcium silicate after it has been formed is also studied, so that the material may be more suitable for use in a variety of applications. 


\section{Chapter 2}

\section{Experimental}

\subsection{Materials}

Throughout this work several different sources of sodium silicate were used (Table 2.1). In each case the grade chosen had a high silica to sodium oxide ratio, a relatively low viscosity to aid handling of the material, and the highest possible concentration of silica provided the previous two criteria had been met. There were no measurable differences in the physical properties of the nano-structured calcium silicates formed according to which sodium silicate source was used.

Table 2.1: Composition details of sodium silicate solutions used.

\begin{tabular}{lccc}
\hline Manufacturer & $\begin{array}{c}\text { Commercial } \\
\text { Grade Name }\end{array}$ & $\begin{array}{c}\% \mathrm{SiO}_{2} \\
(\mathrm{w} / \mathrm{w})\end{array}$ & $\begin{array}{c}\mathrm{SiO}_{2}: \mathrm{Na}_{2} \mathrm{O} \\
(\mathrm{w} / \mathrm{w})\end{array}$ \\
\hline Fernz & $\mathrm{N}$ & 26.3 & 3.2 \\
Orica & $\mathrm{N}$ & 28.7 & 3.2 \\
Crosfields & & 29.0 & 3.3 \\
OxyChem & 40 & 29.2 & 3.22 \\
\hline
\end{tabular}

The hydrochloric acid used for precipitations was an industrial grade supplied by Orica with a concentration of $33 \% \mathrm{w} / \mathrm{w}(10.54 \mathrm{~mol} / \mathrm{L})$. Unless otherwise stated, the calcium hydroxide used was an industrial grade supplied by MacDonald's Lime. Later in the programme a technical grade of calcium hydroxide was used, this was supplied by Loba Chemie. The calcium hydroxide supplied by MacDonald's contained higher proportion of impurities, notably iron oxide that adversely affected the optical properties, and calcium carbonate that adversely affected oil adsorption capacity and surface area of the nanostructured calcium silicate formed (Table 2.2). 
Table 2.2: Major components of the calcium hydroxides used as measured by XRF (weight \%).

\begin{tabular}{|c|c|c|c|c|c|c|c|c|c|}
\hline Supplier & $\mathrm{CaO}$ & MgO & $\mathrm{SiO}_{2}$ & $\mathrm{Al}_{2} \mathrm{O}_{3}$ & $\mathrm{Fe}_{2} \mathrm{O}_{3}$ & LOIa & $\mathrm{Cb}^{\mathrm{b}}$ & Misc. ${ }^{c}$ & Sum \\
\hline Loba Chemie & 74.01 & 0.72 & $<0.01$ & 0.07 & $<0.01$ & 24.68 & 0.2 & 0.17 & 99.64 \\
\hline MacDonald's & 72.04 & 0.62 & 1.03 & 0.31 & 0.30 & 24.92 & 1.33 & 0.29 & 99.50 \\
\hline
\end{tabular}

\subsection{Precipitation of nano-structured calcium silicate}

During the course of this research, two main methods have been employed to produce nano-structured calcium silicate. A batch process was used for earlier research so that preparation variables could be altered easily and because only 2 to $20 \mathrm{~g}$ of dried product was required for analysis. A continuous process was later developed to allow production of larger quantities of dry material (up to $10 \mathrm{~kg}$ ) for applications testing, as well as acting as a model for the most likely method that would be used for commercial production.

\subsubsection{Method for batch production}

Batch production of nano-structured calcium silicate was carried out routinely with slurry volumes ranging from 0.05 to $20 \mathrm{~L}$ using concentrations of monomeric silica varying from 0.072 to $0.57 \mathrm{~mol} / \mathrm{L}$ (significantly above the concentration of geothermal water of ca. $0.01 \mathrm{~mol} / \mathrm{L})$. The batch process enabled higher concentrations to be used than the continuous process as the increased viscosity of slurries with higher \% solids can be problematic for transferring due to a higher likelihood of blocking the tubing. The batch process was therefore used to produce slurries with higher solids content as no pumps were used in this process. The higher viscosity of the slurry produced at higher concentrations did require larger marine-type propellers for stirring and higher torque motors to be employed.

An idealised case is given below as an example of how nano-structured calcium silicate is prepared using the batch method of production. During this study several variables were altered and therefore in these cases the methods used also vary. However, most reactions performed were generally similar to this 
example. The detail of a typical procedure for a $1 \mathrm{~L}$ batch reaction is given in Table 2.3.

Table 2.3: Details of a typical $1 \mathrm{~L}$ reaction.

\begin{tabular}{|c|c|c|c|c|}
\hline & Material & Weight (g) & Volume (mL) & mol \\
\hline \multirow[t]{4}{*}{ A } & Sodium silicate $\left(26.3 \% \mathrm{SiO}_{2}\right)$ & 98.02 & 70.52 & \\
\hline & $\mathrm{SiO}_{2}$ & 25.78 & & 0.4290 \\
\hline & Water & 429.5 & 429.5 & \\
\hline & Total & 527.5 & 500.0 & \\
\hline \multirow[t]{4}{*}{ B } & Calcium hydroxide & 34.73 & 14.97 & 0.4686 \\
\hline & 33\% Hydrochloric acid & 27.94 & 24.00 & 0.2530 \\
\hline & Water & 461.0 & 461.0 & \\
\hline & Total & 523.7 & 500.0 & \\
\hline \multirow[t]{2}{*}{$\mathrm{C}$} & Slurry from A + B & 1051 & 1000 & \\
\hline & Dried product $\left(110^{\circ} \mathrm{C}\right)$ & 62.2 & & \\
\hline
\end{tabular}

The monomeric silica solution (A) was prepared by adding the viscous sodium silicate solution to the distilled water in a $1 \mathrm{~L}$ plastic beaker to give $0.5 \mathrm{~L}$ of solution in total. Glassware was not used for the reactions due to the likely nucleation of monomeric silica on the surface to form a scale. The calcium component for the reaction (B) is made up of calcium hydroxide as the calcium source, and hydrochloric acid to control the $\mathrm{pH}$ during the reaction as the calcium hydroxide was found to cause the slurry to be too alkaline for the formation of a nano-structured calcium silicate with optimal properties. When different calcium salts such as calcium chloride were used, the addition of sodium hydroxide was necessary to increase the $\mathrm{pH}$ above 10 so that the formation of precipitated silica as a result of polymerisation would not occur. Preparation of the acidified calcium precursor slurry requires an acidic solution to be made initially by adding the hydrochloric acid to distilled water in a $1 \mathrm{~L}$ plastic beaker. Hydrochloric acid was chosen as it is a strong acid and because there would not be a problem with the precipitation of a calcium salt as would occur for sulfuric or phosphoric acids. The acid was added at this stage, prior to the reaction, to ensure consistency between batches that could occur with the addition of a separate reagent during the reaction as the initial precipitation 
occurs rapidly. The acid for $\mathrm{pH}$ control is added with the calcium source because direct addition to the sodium silicate solution is likely to cause polymerisation resulting in the formation of a network silica rather than nanostructured calcium silicate. Calcium hydroxide was added to the dilute hydrochloric acid solution whilst constantly stirring the slurry gently in order to keep the calcium hydroxide that had not dissolved in suspension. Due to the impurities in the MacDonald's calcium hydroxide, a larger quantity of this reagent was used to give a molar ratio of calcium to silicon of greater than 1:1 (on the basis that both are pure reagents). By keeping the amount of calcium in excess, the maximum amount of monomeric silica is also removed from the solution and thereby alleviating any concerns about the build-up of silica scale on the equipment.

The sodium silicate solution (A) was added rapidly to the calcium hydroxide slurry (B) which resulted in the immediate precipitation of white particles, noticeable for the increasing whiteness and thickness of the slurry (Figure 2.1). Under typical reaction conditions, greater than $98 \%$ of the calcium and monomeric silica reacted is precipitated as the solid calcium silicate (based upon the concentration calcium cations and monomeric silica remaining in solution). The calcium hydroxide slurry (B) was vigorously stirred during this addition due to the increase in viscosity of the calcium silicate slurry $(\mathrm{C})$ that forms. The resulting $1 \mathrm{~L}$ of viscous nano-structured calcium silicate slurry was kept vigorously agitated for 2 minutes to ensure good mixing during the precipitation followed by gentle agitation for $3 \mathrm{hr}$ during the ageing period whereby the formation of the structure was complete. It was necessary to keep the slurry agitated throughout this ageing period due its thixotropic nature that causes the slurry to set solid if left to stand for an extended period of time. When reactions that used higher overall concentrations were performed, it was necessary to use more vigorous stirring during the initial precipitation phase, as the slurry is more viscous.

The reaction was performed at room temperature without any external heating (although the initial addition of the hydrochloric acid to the calcium hydroxide 
does increase the temperature slightly). The $\mathrm{pH}$ immediately after the reactants had been mixed increased until it reached 12.5 and then dropped over the aging process to finally stabilise at around $\mathrm{pH}$ 12.0. The indicative weight of product given in Table 2.3 is based upon a material that has been water washed and has been dried at $110^{\circ} \mathrm{C}$.

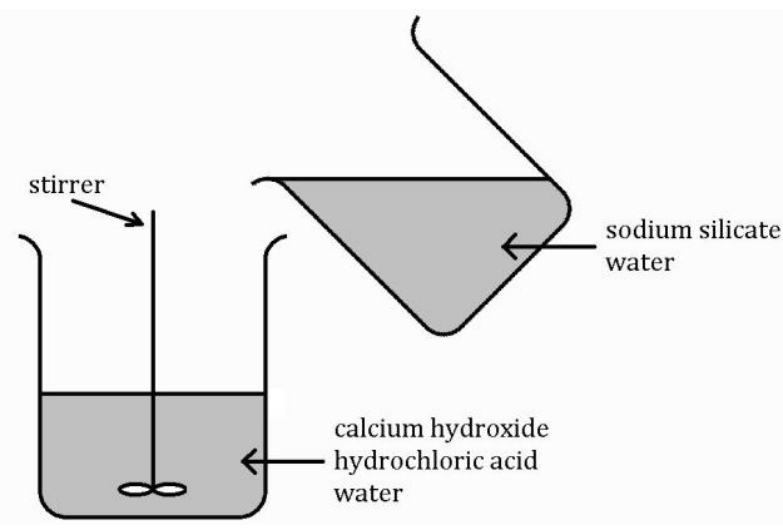

Figure 2.1: Schematic of the batch process.

\subsubsection{Method for continuous production}

Continuous production of nano-structured calcium silicate was carried out in quantities from 1 to $150 \mathrm{~L}$ typically using $0.43 \mathrm{~mol} / \mathrm{L}$ monomeric silica, 0.47 mol/L calcium hydroxide and $0.25 \mathrm{~mol} / \mathrm{L}$ hydrochloric acid as detailed in Table 2.3. At this concentration the slurry formed has a solids content sufficiently high that the volumes used do not become overly large while still being easily pumped and transferred. Preparation of the sodium silicate solution and acidified calcium hydroxide slurry was carried out using the same methods as discussed above in the methodology of the batch process in 2.2.1. Both of these reactants were gently stirred (with particular attention to the calcium hydroxide slurry to prevent any settling occurring) as they were pumped at the same rate by a dual-headed peristaltic pump into a small reaction chamber whereby both could be mixed in a highly controlled environment (Figure 2.2). Typically the reaction vessel was stirred rapidly and the average residence time of the precipitate formed was approximately ten seconds before the slurry overflowed into the large ageing tank that was gently stirred to prevent the thixotropic product from setting. 


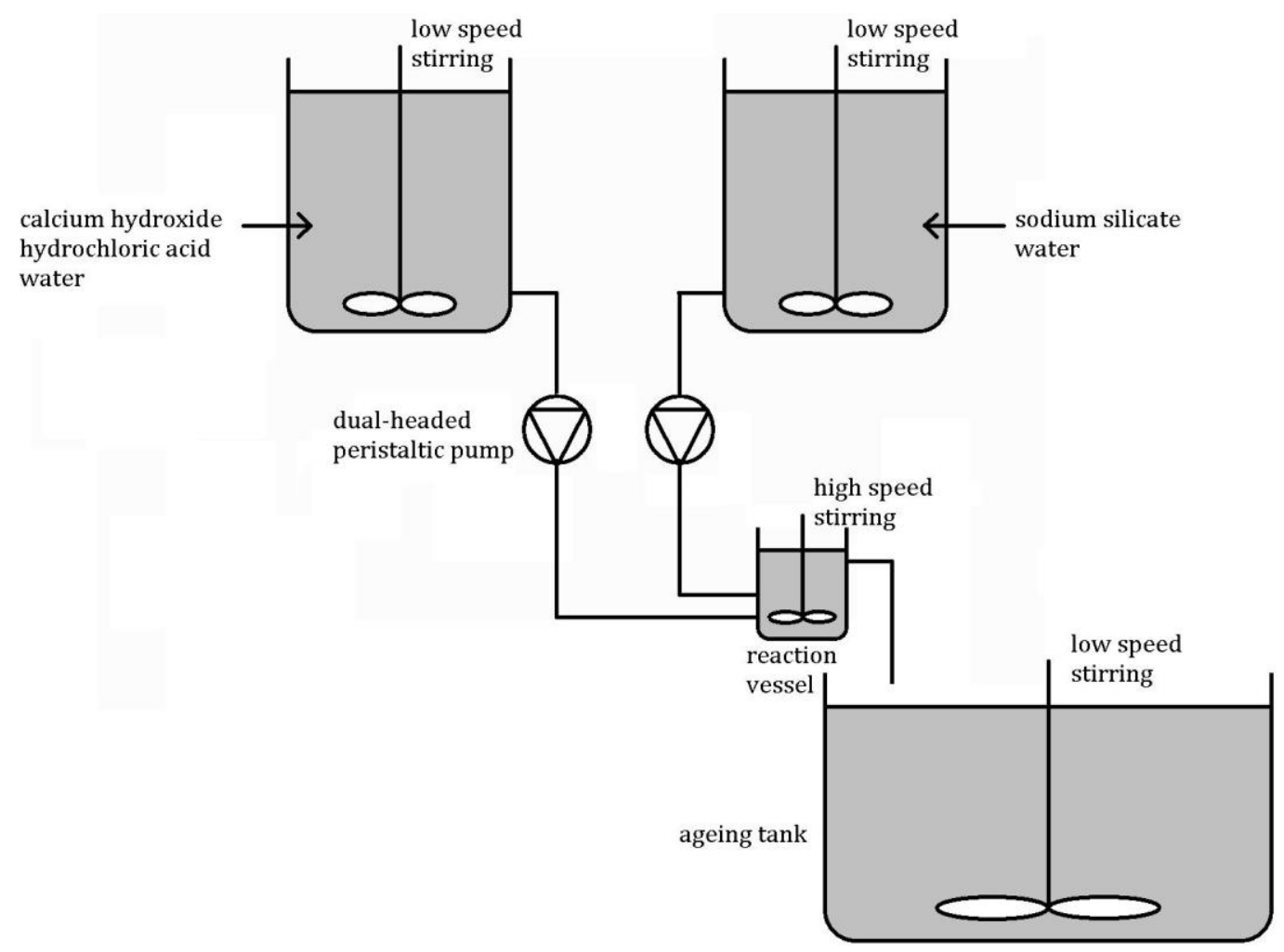

Figure 2.2: A schematic of the continuous process

\subsection{Post-formation treatments}

Once the ageing process whereby the structure develops is completed, four alternative main treatments could be performed to produce materials with different physical properties. Each of these materials, prepared by treating the same intermediate slurry, is suitable for different specific applications. As well as the study of these modified materials for their use in applications, these treatments were also routinely carried out in order to understand the base material such as the development of the structure during the ageing process.

\subsubsection{Washing with water}

Once the ageing process had been completed, the slurry was filtered in a buchner funnel under a water vacuum. Prior to the filter cake cracking as the water was drawn away, three plug flow washes of distilled water individually equivalent in volume to that of the filter cake, were passed through the filter cake consecutively. Plug flow washes are set volumes of solvent that are passed through a filter cake sequentially, with each plug flow completely draining into 
the filter cake before the next is commenced. The purpose of these plug flows was to remove unreacted or unwanted species, such as monomeric silica or sodium and chloride ions, out of the pores of the nano-structured calcium silicate. Depending upon the application, the filter cake was either reslurried in water, or dried at $110{ }^{\circ} \mathrm{C}$ for two days. Upon drying, the material typically shrinks due to the collapse of the open framework structure. Aggregation of the particles causes the material to become somewhat gritty so that the material needs to be ground with an agate mortar and pestle to a fine powder for analysis or for certain applications.

\subsubsection{Washing with 2-ethoxyethanol}

2-ethoxyethanol was used as a spacer compound to prevent collapse of the silicate when drying and therefore enable the pore volume and surface area to be maintained. The washing process is identical to that of washing with water (as in 2.3.1) whereby the slurry is filtered under vacuum and washed with two plug flows of distilled water, but the third plug flow of water is replaced with a single plug flow of 2-ethoxyethanol one and a half times the volume of the filter cake. It is important in this step that as little distilled water as possible from the previous plug flow remains on top of the filter cake before proceeding with this plug flow. This is because water will displace any 2-ethoxyethanol on the surface of the silicate and therefore lead to partial collapse of the structure. Likewise, it is most important to ensure that the 2-ethoxyethanol passes through the whole of the filter cake so that all of the material has been consistently treated, therefore cracking of the filter cake is to be avoided until all of the 2-ethoxyethanol has passed through. The filter cake was then dried in an oven at $110{ }^{\circ} \mathrm{C}$ for two days.

\subsubsection{Reinforcement}

The addition of further silica onto the structure was another method developed as a way to prevent the structure of the silicate collapsing whilst drying. Typical details for this procedure are given in Table 2.4. Sodium silicate was diluted with distilled water and mixed before adding directly to the nano-structured 
calcium silicate slurry whilst stirring. This would then be followed by the steady addition of hydrochloric acid to lower the $\mathrm{pH}$ to 10 and therefore cause the monomeric silica in solution to polymerise onto the surface of the platelets in the framework structure of the silicate. This polymerisation step is very rapid and the slurry could be filtered and washed 5 minutes after the adjustment of the $\mathrm{pH}$. As the material is reinforced to prevent collapse of the structure, the resulting filter cake was just washed with water as in 2.3.1.

Table 2.4: Typical details for the reinforcement of a $1 \mathrm{~L}$ reaction.

\begin{tabular}{|c|c|c|c|c|}
\hline & Material & Weight (g) & Volume (mL) & mol \\
\hline $\mathrm{C}$ & Calcium silicate slurry $(A+B)$ & 1051 & 1000 & \\
\hline \multirow[t]{4}{*}{$\mathrm{D}$} & Sodium silicate $\left(26.3 \% \mathrm{SiO}_{2}\right)$ & 70.00 & 50.36 & \\
\hline & $\mathrm{SiO}_{2}$ & 18.41 & & 0.3064 \\
\hline & Water & 449.6 & 449.6 & \\
\hline & Total & 519.6 & 500.0 & \\
\hline $\mathrm{E}$ & $C+D$ & 1571 & 1500 & \\
\hline $\mathrm{F}$ & 33\% Hydrochloric acid & 22.08 & 18.97 & 0.2000 \\
\hline \multirow[t]{2}{*}{ G } & $E+F$ & 1593 & 1519 & \\
\hline & Dried product $\left(110^{\circ} \mathrm{C}\right)$ & 76 & & \\
\hline
\end{tabular}

\subsection{4 pH adjustment}

For some applications it is necessary for the nano-structured calcium silicate produced to be less alkaline. In these cases the $\mathrm{pH}$ of the slurry was adjusted by adding 33\% hydrochloric acid slowly until the desired pH was reached. It was deemed necessary to use concentrated acid due to the buffering effect of the material resulting in relatively large quantities of acid to be added to reach the pH required in some cases. For lower concentration reactions or material produced on a smaller volume the acid was diluted so that a more easily measured volume could be used. As nano-structured calcium silicate is soluble below pH 6 it is necessary to have effective stirring for this process and to add the acid in a slow and controlled manner (such as using a burette for the addition) to prevent localised regions of low $\mathrm{pH}$ in the slurry. A $1 \mathrm{~L}$ reaction 
required $0.10 \mathrm{~L}$ of $33 \%$ hydrochloric acid to be added to reach $\mathrm{pH} 7$, with the adjustment taking 24 hours before equilibrium was reached due to buffering.

\subsection{Characterisation methods}

\subsection{1 pH measurement}

The pH was measured with Hanna Instruments pH meters models HI 8521 (bench top) or HI 9025C (portable), using HI 1230 double junction combination electrodes (with $\mathrm{Ag} / \mathrm{AgCl}$ reference electrode). The $\mathrm{pH}$ meter was usually calibrated by the use of both pH 7 and pH 10 buffers as most solutions analysed were alkaline. The need to extrapolate readings above this range as the majority of solutions measured were more alkaline than $\mathrm{pH} 10$, decreases the accuracy of $\mathrm{pH}$ values measured of these solutions. As a result of being frequently immersed in solutions that contained monomeric silica, deposition of silica onto the glass bulb of the electrode would occur over time and result in increased response times. It was therefore necessary to periodically replace the electrode when its performance deteriorated noticeably.

\subsubsection{Monomeric silica concentration analysis}

The concentration of monomeric silica in solution was determined using the silicomolybdate method recommended by Iler (1979). This method involves the formation of yellow silicomolybdic acid due to the reaction between acidified ammonium heptomolybdate and monomeric silica (and not polymerised forms), therefore allowing the concentration to be determined spectrophotometrically (see equation below).

$$
7 \mathrm{Si}(\mathrm{OH})_{4}+12 \mathrm{H}_{6} \mathrm{Mo}_{7} \mathrm{O}_{24 .} 4 \mathrm{H}_{2} \mathrm{O}+126 \mathrm{H}_{2} \mathrm{O} \rightleftharpoons 7 \mathrm{H}_{8} \mathrm{Si}\left(\mathrm{Mo}_{2} \mathrm{O}_{7}\right)_{6} .28 \mathrm{H}_{2} \mathrm{O}
$$

As the dilute molybdic acid reagent solution is only stable for around one week, this solution is made up when required from two more stable solutions as detailed below. 
Reagent A: Add $41.0 \mathrm{~mL} 95.5 \%(\mathrm{w} / \mathrm{w}$ ) sulphuric acid to $800 \mathrm{~mL}$ double distilled water and dilute to $1 \mathrm{~L}$.

Reagent B: Dissolve $100 \mathrm{~g}$ of ammonium molybdate(VI) tetrahydrate in 900 $\mathrm{mL}$ double distilled water, add $47 \mathrm{~mL} \mathrm{28 \%} \mathrm{(w/w)} \mathrm{ammonium} \mathrm{hydroxide,} \mathrm{and}$ dilute to $1 \mathrm{~L}$.

Reagent C: $\quad$ To $500 \mathrm{~mL}$ double distilled water add $200 \mathrm{~mL}$ of reagent $\mathrm{A}$ and $100 \mathrm{~mL}$ of reagent $\mathrm{B}$.

A 10 to $20 \mathrm{~mL}$ sample was taken from the reaction slurry via a syringe and filtered through a $0.65 \mu \mathrm{m}$ cellulose nitrate filter attached at the end of the syringe. An aliquot of this solution was pipetted into a $50 \mathrm{~mL}$ volumetric flask, $40 \mathrm{~mL}$ of reagent $\mathrm{C}$ was added using a 10 to $50 \mathrm{~mL}$ dispensette from Merck and the volume made up to $50 \mathrm{~mL}$ with double distilled water.

The beta form of silicomolybdic acid that develops initially is more coloured but less stable than the alpha form, therefore the absorbance is measured ca. 10 minutes (Harper, 1994) after reagent $C$ was added to the sample solution. This time period is sufficient to allow the colour to fully develop, but before the colour starts to fade appreciably. The absorbance of the solutions was measured at $410 \mathrm{~nm}$ using a Shimadzu UV-160 spectrophotometer operated in concentration mode after the measurement of two standard solutions. One standard solution was a blank sample prepared by auto-dispensing $40 \mathrm{~mL}$ of reagent $C$ into a $50 \mathrm{~mL}$ volumetric flask and making the solution up to $50 \mathrm{~mL}$ with double distilled water. A $100 \mathrm{mg} / \mathrm{L}$ silicon standard was prepared from a Spectrosol BDH $1000 \mathrm{mg} / \mathrm{L}$ silicon solution of sodium metasilicate. A $5 \mathrm{~mL}$ aliquot of the $100 \mathrm{mg} / \mathrm{L}$ silicon standard was added to a $50 \mathrm{~mL}$ volumetric flask followed by $40 \mathrm{~mL}$ of reagent $\mathrm{C}$ via an auto-dispenser and then made up to 50 $\mathrm{mL}$ with double distilled water. All sample solutions were diluted so that the silicon concentrations were between these two standards. The silicon concentration of the samples displayed by the instrument (in $\mathrm{mg} / \mathrm{L}$ ) was then converted into a concentration of silica in the original solution (mg/L) using the following formula. 


$$
\left[\mathrm{SiO}_{2}\right]_{m}=[\mathrm{Si}] \times \frac{\mathrm{M}_{\mathrm{r}}\left(\mathrm{SiO}_{2}\right)}{\mathrm{A}_{\mathrm{r}}(\mathrm{Si})} \times \frac{\text { Standard aliquot volume }}{\text { Sample aliquot volume }}
$$

The combined uncertainty in results from both the tolerances of equipment used and spectrometer drift is $\pm 3 \%$.

\subsubsection{Atomic absorption spectroscopy}

The concentration of calcium ions in solution was determined with a GBC 906 AA atomic absorption spectrometer. It was likely that during the atomisation process the presence of soluble silicate anions in solution would cause the formation of a stable calcium silicate that is not broken down in the cooler airacetylene flame, which would therefore cause depressive effects to be observed. Measurements were therefore made using the hotter nitrous oxideacetylene flame which required a $2000 \mathrm{mg} / \mathrm{L}$ potassium ionisation buffer to be added to all samples. The ionisation buffer was made by dissolving $3.813 \mathrm{~g}$ of potassium chloride (AR) with double distilled water and making the volume up to $1 \mathrm{~L}$. Operating conditions followed those in the instrument's manual (Athanasopoulos, no year given) with an oxidising nitrous oxide-acetylene flame, $422.7 \mathrm{~nm}$ wavelength used, and all solutions diluted so that the calcium concentration was within the optimum working range of 1 to $4 \mathrm{mg} / \mathrm{L}$. Four standard solutions of $1,2,3$, and $4 \mathrm{mg} / \mathrm{L}$ and a blank ( $0 \mathrm{mg} / \mathrm{L})$ were used to determine the calibration curve, with all solutions made up with the ionisation buffer. The four $0.1 \mathrm{~L}$ standards were prepared from a $1000 \mathrm{mg} / \mathrm{L}$ calcium standard that was made according to the manual whereby $2.497 \mathrm{~g}$ of dried calcium carbonate (AR) was dissolved with $0.025 \mathrm{~L}$ of $1 \mathrm{~mol} / \mathrm{L}$ hydrochloric acid (AR) and diluted to $1 \mathrm{~L}$ with double distilled water. As the concentration of the solutions measured can differ greatly, each sample requires the use of different equipment to dilute the concentration to within the instruments optimum working range, therefore the uncertainty of the results vary between individual samples. An estimate of the uncertainty due to tolerances of equipment used is $2 \%$. The instrument itself also calculates the uncertainty for the measurement of each concentration determined, based upon the linearity of 
the calibration curve used as well as the variation between the three readings taken of each sample.

\subsubsection{Particle size analysis}

Particle size data was measured on a Malvern Mastersizer Hydro 2000 MU. Samples for analysis were preferably in a wet slurry form due to the aggregation that occurs upon drying. The slurry was added to $800 \mathrm{~mL}$ of distilled water until the laser had ca. $10 \%$ obscurity. The solution was then sonicated by the instrument's built-in ultrasonic probe for 60 seconds with the tip displacement set at $10 \mu \mathrm{m}$. The measurements were made over 20 seconds (therefore taking 20000 snapshots) using a pump speed of $2000 \mathrm{rpm}$. Calculations were based on material properties of a reflective index 1.61 and an absorption of 0.1 on Malvern Mastersizer 2000 software v 5.1.

\subsubsection{Transmission electron microscopy}

A Philips 420ST high resolution transmission electron microscope at the Electron Microscope Facility at Victoria University was used with either a tungsten or lanthanum boride filament during the course of the work. Samples were prepared for analysis by adding a few drops of slurry to ca. $20 \mathrm{~mL}$ of distilled water and sonicating for one minute in an ultrasonic bath to break up any aggregates. A sample still in the wet-slurry state was preferred as the degree of aggregation is much less than for a sample that had been dried. When it was necessary to analyse a dried sample, the powder was added to ca. $1 \mathrm{~mL}$ of distilled water and wet-ground using a mortar and pestle before diluting to ca. $20 \mathrm{~mL}$ and sonicating for 2-5 minutes depending upon the degree of aggregation. A fraction of a drop of the diluted and sonicated sample was placed onto a holey carbon coated copper grid by using a pasteur pipette and then dried under a $50 \mathrm{~W}$ light bulb.

\subsubsection{Scanning electron microscopy}

High-resolution scanning electron micrographs were obtained using a Philips XL 30S FEG-SEM at the University of Auckland. Samples were prepared by 
finely grinding the material dried at $110^{\circ} \mathrm{C}$ for two days, using an agate mortar and pestle, and then lightly dusting the powdered sample onto a carbon disk. The sample was then typically coated with platinum to reduce the amount of charging. Some earlier samples were carbon coated, although this resulted in the loss of some detail at higher magnifications.

\subsubsection{Oil absorption}

The oil absorption was determined according to ASTM D281-31 1980, Oil Absorption of Pigments by Spatula Rub-Out test method. This test provides a quick and simple measure of the oil absorption capacity of a powder. The method consists of working raw linseed oil into the ground silicate powder with a spatula, with the end-point being achieved just before the powder becomes a paste. Before the end-point is reached the material remains as discrete aggregates, but when the end-point is passed the material becomes a paste as the oil has filled the particles internal voids and the excess oil coats the exterior of the particles and binds them together. The oil absorption capacity is determined by weighing the mass of oil needed to reach the end-point of a specific amount of silicate, with the industry standard for reporting the oil absorption capacity as weight of oil absorbed (g) per $100 \mathrm{~g}$ of absorbent. Raw linseed oil was preferred over bis (2-ethylhexyl) phthalate as it is less toxic and therefore more suitable as a readily used bench-top test. The use of linseed oil provided stable measurements over time if care was taken to seal containers and keep them away from direct sunlight to prevent oxidation of the oil. Samples for analysis were dried in an oven at $110{ }^{\circ} \mathrm{C}$ for at least two days after filtration before being finely ground using a mortar and pestle and being returned to the oven for at least 30 minutes before analysis. Under these drying conditions the majority of the unbound water within the material can be removed to a consistent extent and therefore allow consistent oil absorption measurements to be made. The spatula rub-out test was performed in duplicate on 0.250 to $1.000 \mathrm{~g}$ samples (depending upon the absorptive capacity of the material) or until concurrent results were obtained. The test was performed immediately after removing the sample from the oven due to the somewhat 
hygroscopic nature of the material (Figure 2.3). Also, due to the gradual substitution of 2-ethoxyethanol by moisture in the air and the subsequent irreversible collapse of the structure that this causes, samples that had been treated in this way needed to be tested after the initial drying process and not after re-drying following storage. Results obtained using this test can vary noticeably between users as the differing amounts of pressure applied by individuals over different time periods alter the observed end point. However, an individual is able to achieve reproducible results over time by performing the test consistently on each sample. The uncertainty for this test, given that it will not always be applied with exactly the same technique as well as the materials propensity to adsorb moisture from the atmosphere, is estimated to be $\pm 4 \%$.

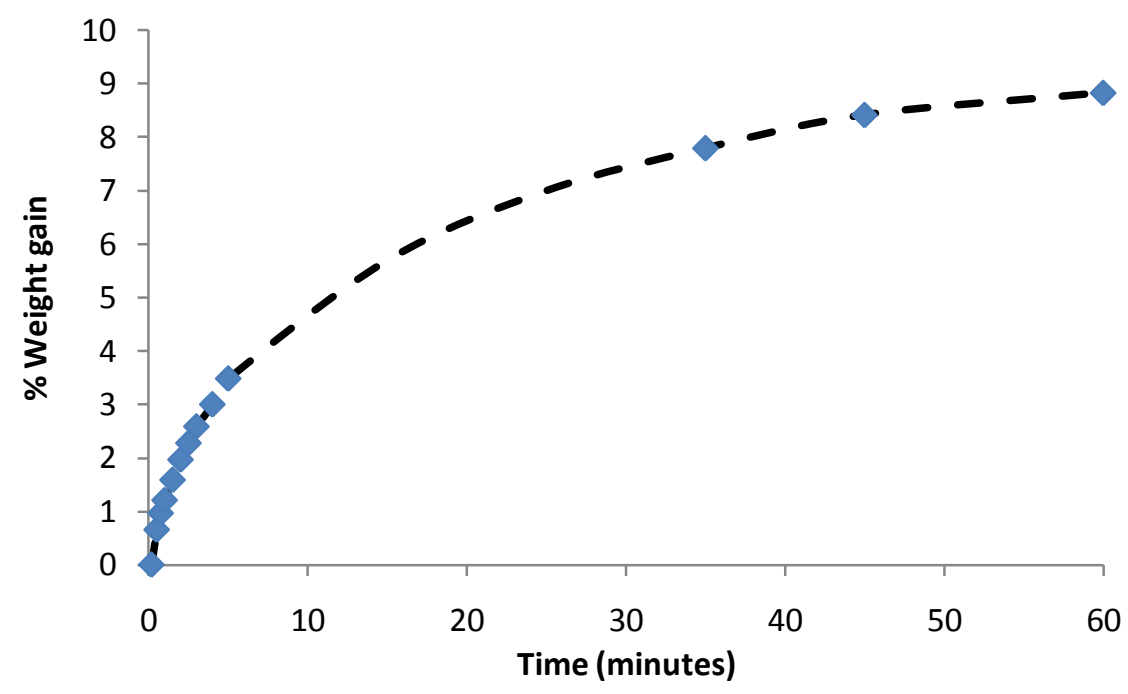

Figure 2.3: Weight gain as a result of the uptake of atmospheric moisture by nano-structured calcium silicate under typical laboratory humidity and temperature conditions.

\subsubsection{Surface area}

The surface area was determined on a Micromeritics Flowsorb II by the BET method according to ASTM D 45567-86 Standard Test Method for Single Point Determination of Specific Area of Catalysts Using Nitrogen Adsorption by Continuous Flow Method and manuals provided with the instrument. The methodology in the manual was not followed for the calculation of the specific 
surface area as a modification of the original BET equation (Meyer, 1996) was preferred (see below).

$$
\begin{aligned}
& \text { Specific Surface Area }=\left(\frac{\mathrm{C}_{1}}{\mathrm{~W}_{s}}\right) \times\left(\frac{295}{\mathrm{~T}_{\mathrm{a}}}\right) \times\left(\frac{\mathrm{P}_{\mathrm{a}}}{760}\right) \times\left(\frac{1-\left(\mathrm{P} / \mathrm{P}_{0}\right)}{0.706}\right)
\end{aligned}
$$

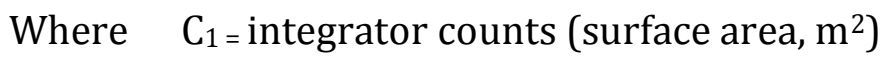

$$
\begin{aligned}
& \mathrm{W}_{\mathrm{s}}=\text { sample weight }(\mathrm{g}) \\
& \mathrm{T}_{\mathrm{a}}=\text { ambient temperature }(\mathrm{K}) \\
& \mathrm{P}_{\mathrm{a}}=\text { ambient pressure }(\mathrm{mm} \mathrm{Hg}) \\
& \mathrm{P}=\text { partial pressure of } \mathrm{N}_{2}(\mathrm{~mm} \mathrm{Hg}) \\
& \mathrm{P}_{0}=\text { saturated equilibrium vapour pressure of liquid } \\
& \text { nitrogen (assumed to be } 15 \mathrm{~mm} \mathrm{Hg} \text { ) }
\end{aligned}
$$

This equation allows for the correction due to temperature and pressure deviation from standard conditions, to be made during the post-measurement calculation rather than during instrument calibration. As recommended in the instrument's manual the integrator counts for the desorption of the sample was always used for calculating the specific surface area. A ca. 30\% $\mathrm{N}_{2} / 70 \% \mathrm{He}$ instrument grade gas mixture was used for all measurements and samples for analysis were dried in an oven at $110{ }^{\circ} \mathrm{C}$ for at least two days after filtration before being finely ground using a mortar and pestle. As in the case of oil absorption tests, care had to be taken that samples treated with 2ethoxyethanol were not exposed for a prolonged period of time to moisture in the air due to the irreversible collapse of the structure that would result. The surface area was therefore always determined following the initial drying of the sample and the samples spent as little time as possible outside of the oven during the grinding process. All samples were degassed under the $30 \% \mathrm{~N}_{2} 70 \%$ He gas mixture at $105^{\circ} \mathrm{C}$ for 1 hour immediately prior to the measurement as this time period had been determined to give consistent results. Typically, samples masses of 0.020 to $0.100 \mathrm{~g}$ gave surface area readings within the optimum range of the instrument. The accuracy of the instrument is typically better than $\pm 3 \%$ for low specific surfaces and $\pm 2 \%$ for moderate to high 
specific surfaces using the single point method, with $\pm 0.5 \%$ reproducibility of results (as listed in Flowsorb II 2300 Instruction Manual, 1990).

\subsubsection{Thermogravimetric analysis}

Thermogravimetric analysis was performed on a Shimadzu TGA-50H under the typical conditions of an air atmosphere flowing at $10 \mathrm{~mL} / \mathrm{min}$, using a platinum pan and a heating rate of $5{ }^{\circ} \mathrm{C} / \mathrm{min}$. Due to the hygroscopic nature of the material, samples for analysis were removed from the oven immediately prior to the test to reduce variability due to the adsorption of water at differing atmospheric humidities.

\subsubsection{Powder X-ray diffraction}

X-ray diffraction patterns were recorded using a Phillips PW3710 analytical Xray diffractometer, with a graphite monochromator and copper anode operating at $40 \mathrm{kV}$ and $20 \mathrm{~mA}$ using the $\mathrm{K} \alpha_{1}$ wavelength $\lambda=1.54056 \mathrm{~nm}$. Diffraction patterns were typically recorded from 10 to $70^{\circ} 2 \theta$ at a scanning rate of $1^{\circ}$ per minute. Samples for analysis had been dried at $110{ }^{\circ} \mathrm{C}$ and finely ground with a mortar and pestle.

\subsubsection{X-ray fluorescence}

X-ray fluorescence analysis was used to determine the elemental composition of the nano-structured calcium silicate samples with the main interest focussing on the proportion of silicon to calcium particularly for post-formation modifications. Results were obtained from either the Analytical Facility at Victoria University on a Philips PW1404 Automatic Sequential X-ray Spectrometer using the conventional fused disc method for major element determination of rock and mineral samples, or from SpectraChem Analytical Ltd. 


\subsubsection{2 ${ }^{29} \mathrm{Si}$ Nuclear magnetic resonance}

${ }^{29} \mathrm{Si}$ nuclear magnetic resonances were recorded under magic angle spinning at 11.7 $\mathrm{T}$ using a Varian Unity 500 spectrometer and a $5 \mathrm{~mm}$ Doty high-speed probe spun at 10 to $12 \mathrm{~Hz}$ with tetramethylsilane used as an external standard. The spectrometer conditions were: $6 \mu$ s, $\pi / 2$ pulse, and a recycle delay of typically 300 seconds to allow for the presence of slow-relaxing species.

\subsubsection{Optical properties}

The optical properties of nano-structured calcium silicate are important for some applications such as when the material is used as either a filler or a part of a coating formulation in paper. The optical properties were determined by pressing the dry powder into a disc, and measuring the reflectance at $457 \mathrm{~nm}$ (TAPPI brightness) as well as $\mathrm{L}^{*}, \mathrm{a}^{*}$, and $\mathrm{b}^{*}$ values on the CIE scale with a HunterLab ColorQuest colourimetric spectrophotometer with daylight 65 as the illuminant and an observer angle of $10^{\circ}$ (HunterLab, 1996). The CIELAB colour scale quantifies human colour perception by acquiring measurements through mimicking the way that the human eye functions and the use of a 3 dimensional colour space (Figure 2.4). The scale is based upon the opponentcolours theory that uses the way in which the human visual system interprets information about colour. The theory states that the response to a colour in one colour channel is antagonistic to the other colour of the channel, where the three opponent channels are red versus green, blue versus yellow, and black versus white (HunterLab, 2001). The 3-dimensional colour space provides an approximately uniform colour scale whereby the axes are:

L (lightness) axis $\quad-0$ is black, 100 is white (a perfect reflecting diffuser)

a (red-green) axis - positive values are red, negative values are green

b (blue-yellow) axis - positive values are yellow, negative values are blue

The optical properties measured using the CIELAB colour scale are given as a ratio whereby the difference between a sample and a standard is determined so 


\section{CHAPTER 2: EXPERIMENTAL}

that colour values can be universally compared. As such, the a and $\mathrm{b}$ axes have no specific numerical limits and all values can be said to have relative units.

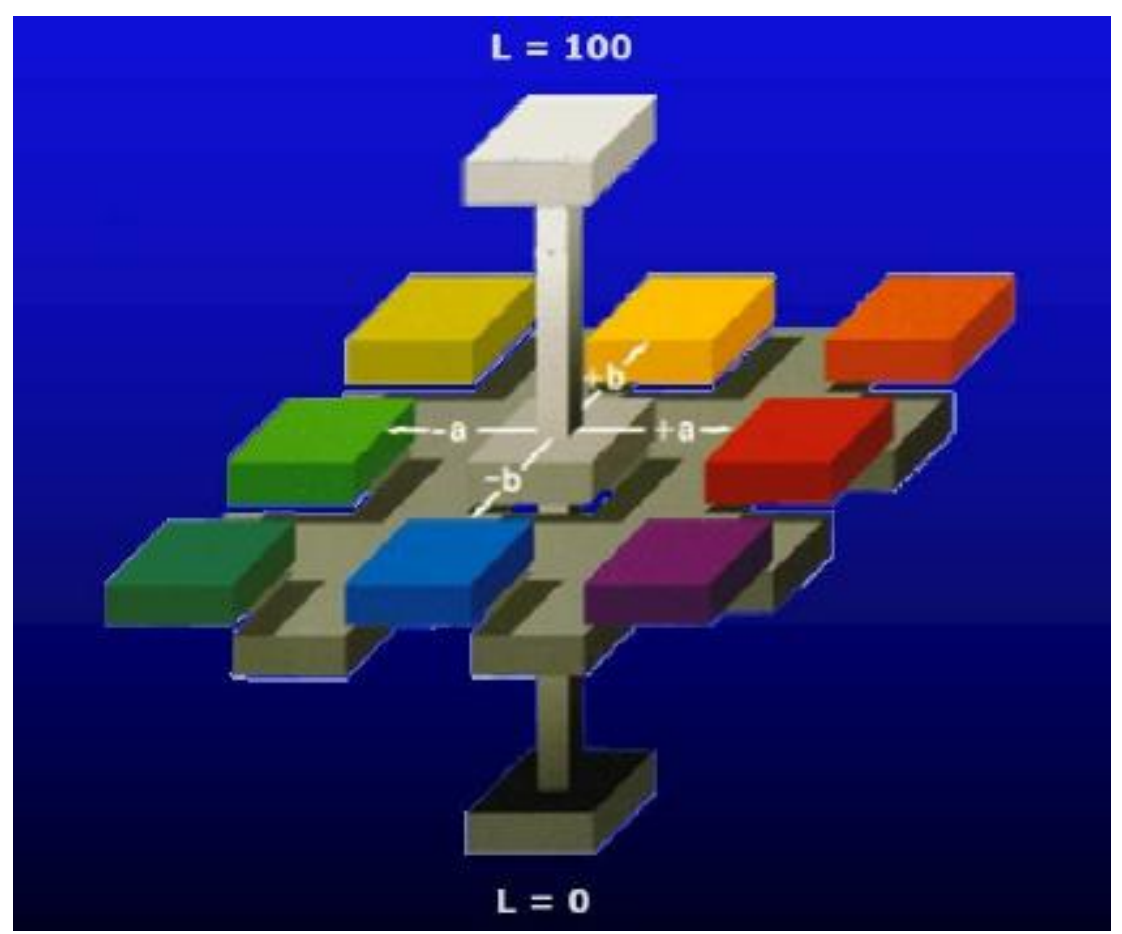

Figure 2.4: A representation of the CIELAB colour space (HunterLab, 2001). 


\section{Chapter 3}

\section{FORMATION OF NANO- STRUCTURED CALCIUM SILICATE}

The formation of nano-structured amorphous calcium silicate can be described as taking place in two stages. The first stage is the immediate precipitation of the hydrated calcium silicate, which is then followed by a second slower ageing process whereby the open framework structure is developed.

\subsection{The precipitation process}

This process is a result of the supersaturated solution that exists once the reactants are mixed together. The precipitation of calcium silicate occurs essentially instantaneously due to the low solubility of the material, as can be observed in Figure 3.1 by measuring the concentration of monomeric silica in solution. The monomeric silica concentration at the point of first sampling just 5 minutes into the reaction of $5 \mathrm{mg} / \mathrm{L}$, is very close to the final concentration measured after 24 hours of $15 \mathrm{mg} / \mathrm{L}$. The concentration after 5 minutes is significantly lower than the concentration of silica initially added of 19200 $\mathrm{mg} / \mathrm{L}(0.319 \mathrm{~mol} / \mathrm{L})$ indicating the speed of the reaction. Only a fraction of the $12500 \mathrm{mg} / \mathrm{L}$ (0.313 mol/L) calcium added for the reaction is present as ions in solution due to the insolubility of calcium hydroxide at room temperature. Based upon the solubility of calcium hydroxide specified by Lide (2005), the concentration of calcium ions in solution would be $0.022 \mathrm{~mol} / \mathrm{L}$ (ca. $870 \mathrm{mg} / \mathrm{L}$ ). However, at the first time of sampling the measured calcium concentration of $420 \mathrm{mg} / \mathrm{L}$ is below the solubility of calcium hydroxide. Gradually, after ca. 2 hours the calcium ion concentration stabilised as it approached the final concentration measured (after 24 hours) of $180 \mathrm{mg} / \mathrm{L}$. It has been found (Roller \& Ervin, 1940; Fujii \& Kondo, 1981) that five days contact with water was 
sufficient for a steady state to be achieved for calcium silicate hydrate gels and therefore the material may not be quite at equilibrium after 1 day.

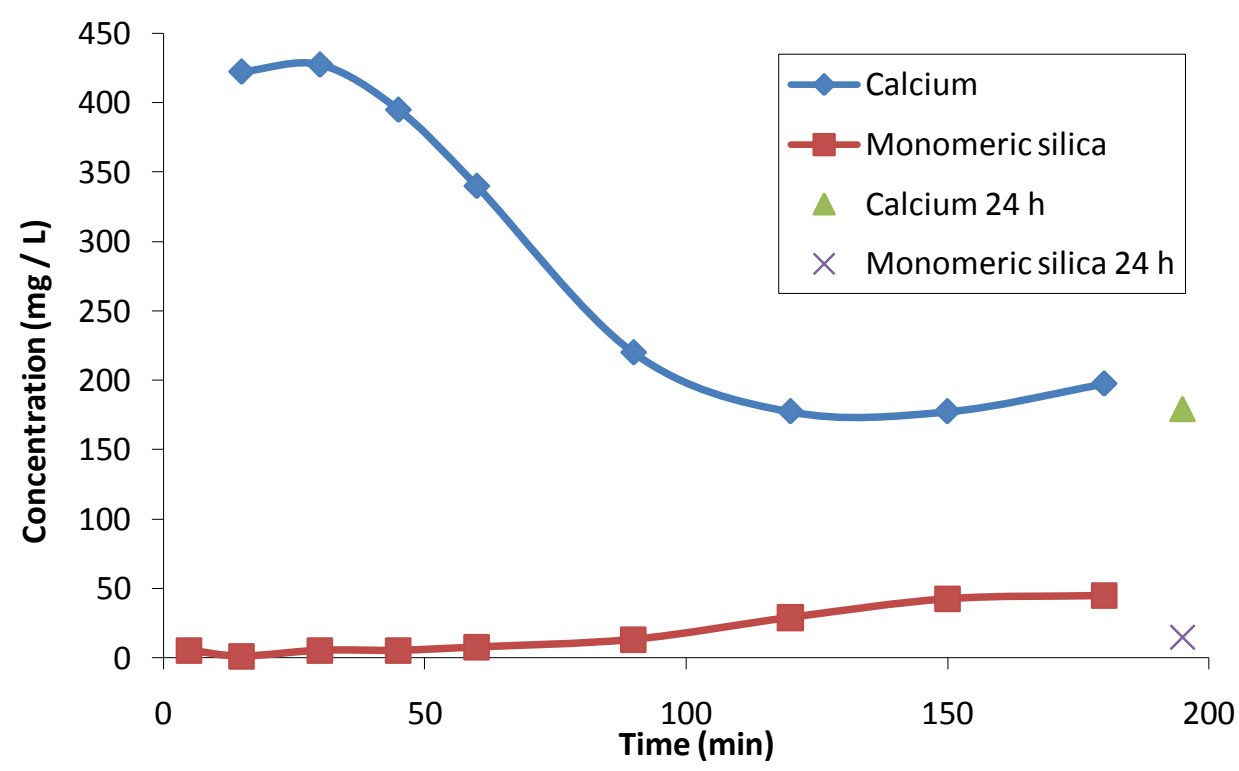

Figure 3.1: Change in the concentration of the reactants remaining in solution during the ageing process.

Like microcrystalline silicas and other hydrated calcium silicates, it is probable that the solubility of the nano-structured calcium silicate is dependent upon the ultimate particle size, specific surface area, and degree of order in the structure, as well as $\mathrm{pH}$, temperature and pressure. The solubility of crystalline calcium metasilicate $(\alpha)$ is $0.0095 \mathrm{~g} / 100 \mathrm{ml}$ water (Lide, 2005). The Ca/Si ratio of the solid formed as determined by XRF remains essentially constant throughout the ageing process (Figure 3.2), indicating that the composition of the initially formed precipitate does not change during the development of the structure. However, these measurements are performed upon the bulk material and it is not possible to determine with this technique if there are compositional changes occurring at a micro-scale.

Due to the rapid nature of this initial precipitation, the most important feature of the initial stage in terms of process design was to ensure that the reactants were well mixed quickly to allow the precipitation to occur between species at ideal relative concentrations and without local concentration gradients within the slurry. 


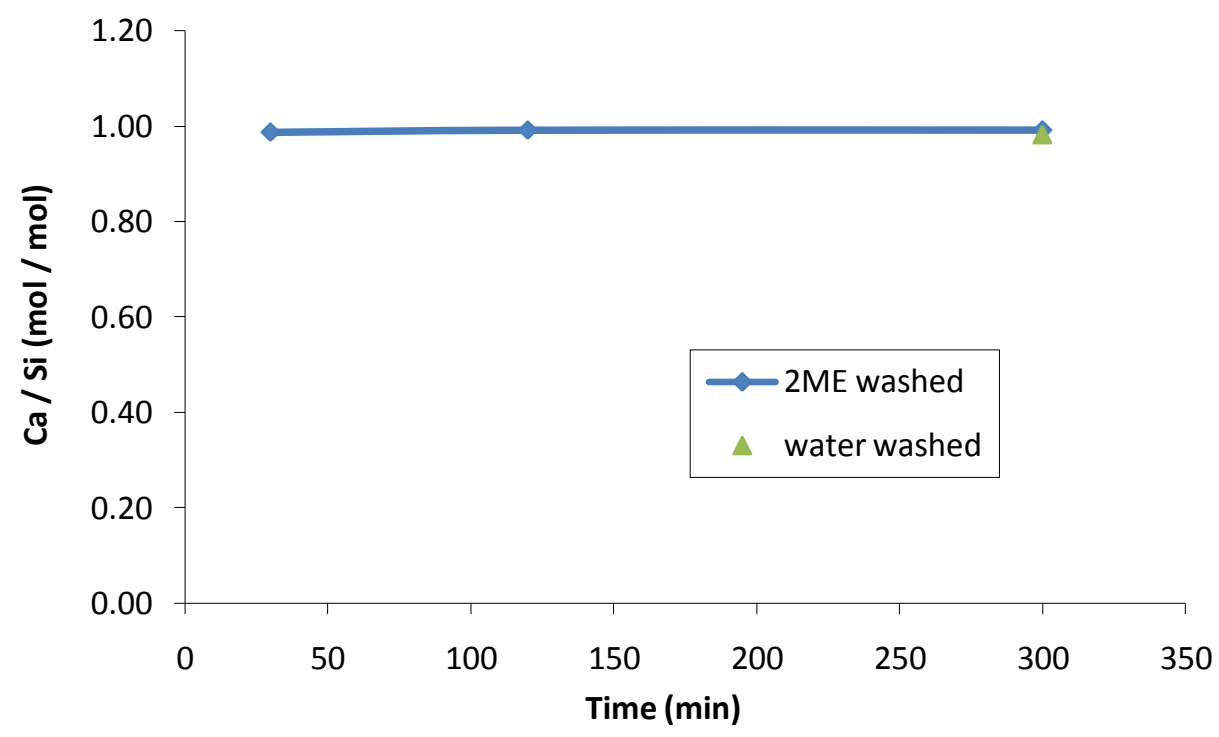

Figure 3.2: Composition of solids during the ageing process as determined by XRF (2ME is 2methoxyethanol).

\subsection{The ageing process}

The result of the initial precipitation is a material that does not exhibit the desert-rose structure or contain any platelets whatsoever, as can be seen in Figure 3.3. The formation of the open framework structure occurs during the ageing stage whereby the material with a globular network-like structure formed initially upon precipitation is self-organised into platelets that eventually constitute all of the material. It can be seen in Figure 3.4 that 10 minutes after precipitation that there are some small areas of platelets present in the material (in the circled areas). The proportion of platelets increases as can be seen in Figure 3.5 and Figure 3.6 during this ageing process until eventually the desert-rose structure completely dominates (Figure 3.7). As well as an increasing proportion of platelets in the material during the ageing process, the size of the platelets also increases as can be seen by comparing the structure after 60 minutes with that after 300 minutes. The variation in the size and shape of the platelets can be more easily seen in Figure 3.8 where a sample that has been aged contains platelets of different thicknesses and degrees of curvature. This lack of uniformity is likely to be a result of the disorder within the atomic structure of the material. 


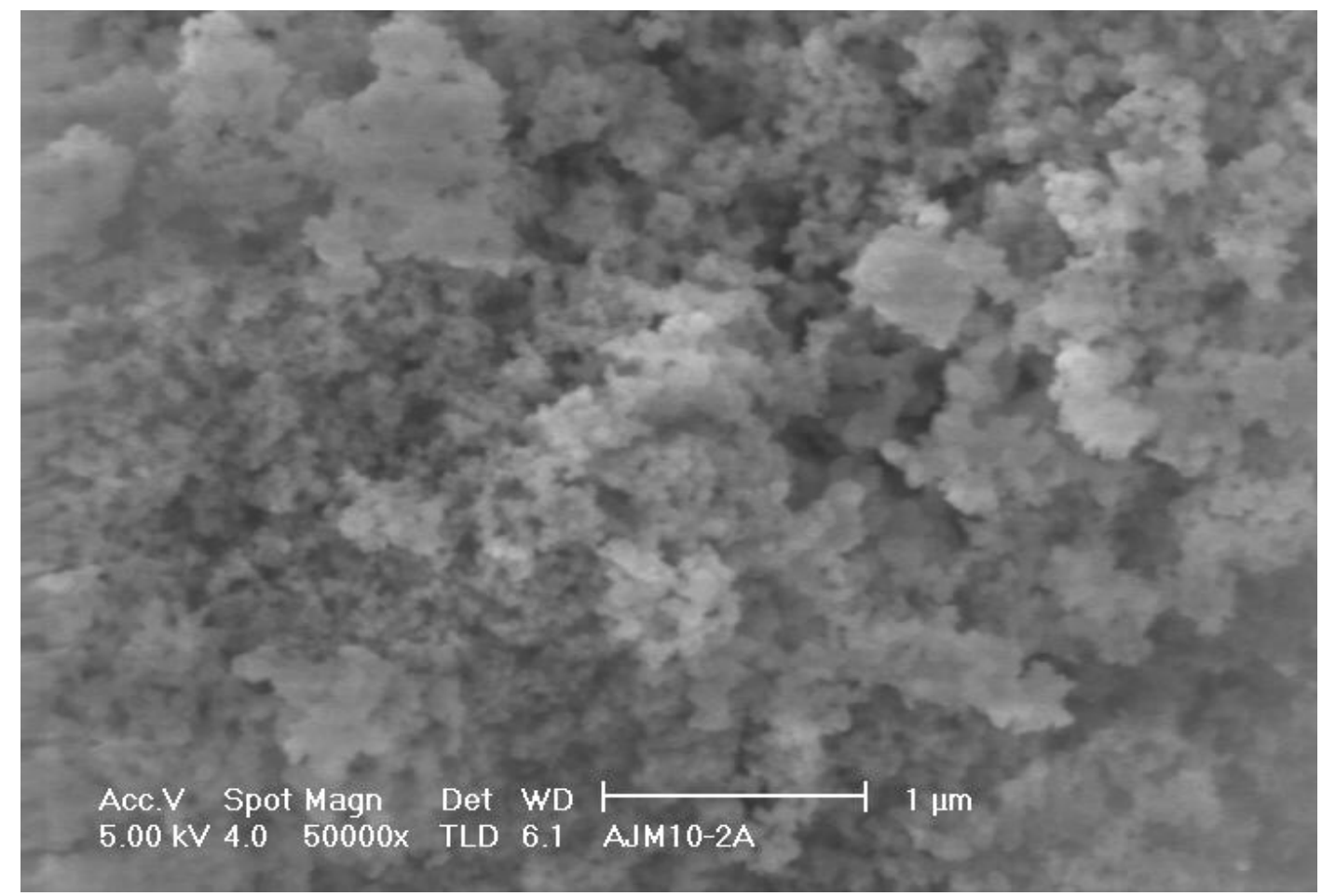

Figure 3.3: Scanning electron micrograph of 2-ethoxyethanol treated nano-structured calcium silicate 2 minutes after precipitation.

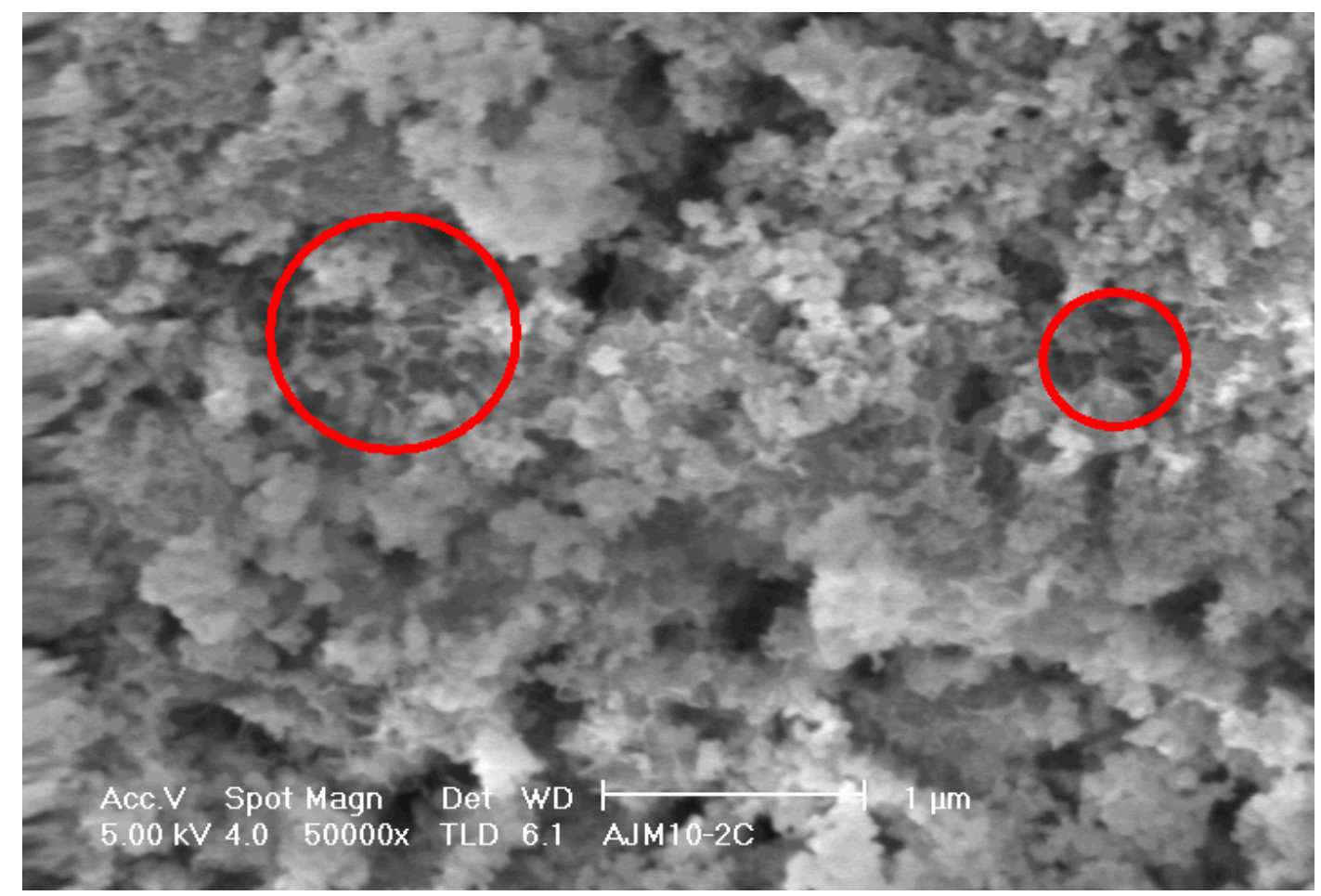

Figure 3.4: Scanning electron micrograph of 2-ethoxyethanol treated nano-structured calcium silicate 10 minutes after precipitation with the first appearance of platelets circled. 


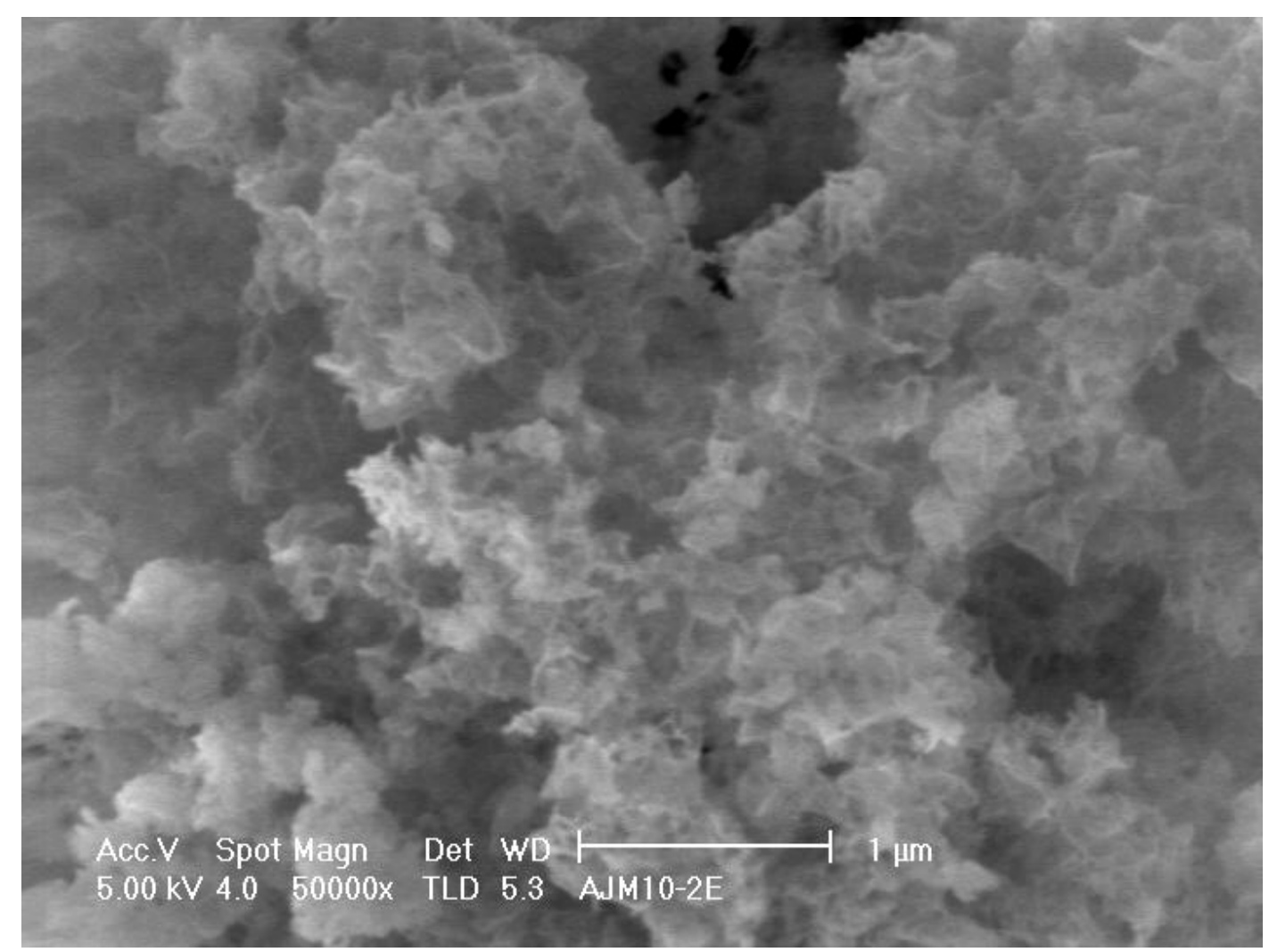

Figure 3.5: Scanning electron micrograph of 2-ethoxyethanol treated nano-structured calcium silicate 60 minutes after precipitation.

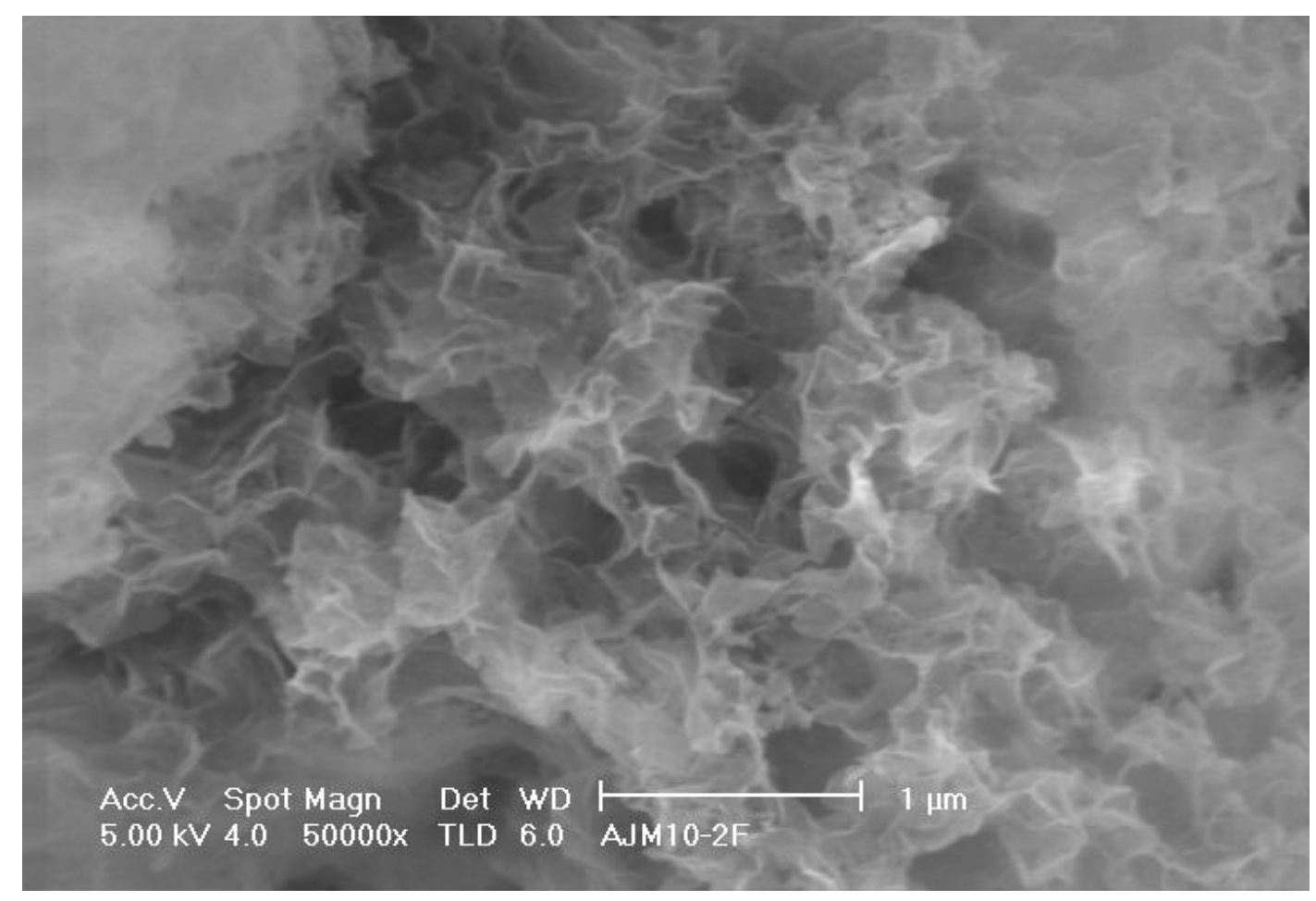

Figure 3.6: Scanning electron micrograph of 2-ethoxyethanol treated nano-structured calcium silicate 150 minutes after precipitation. 


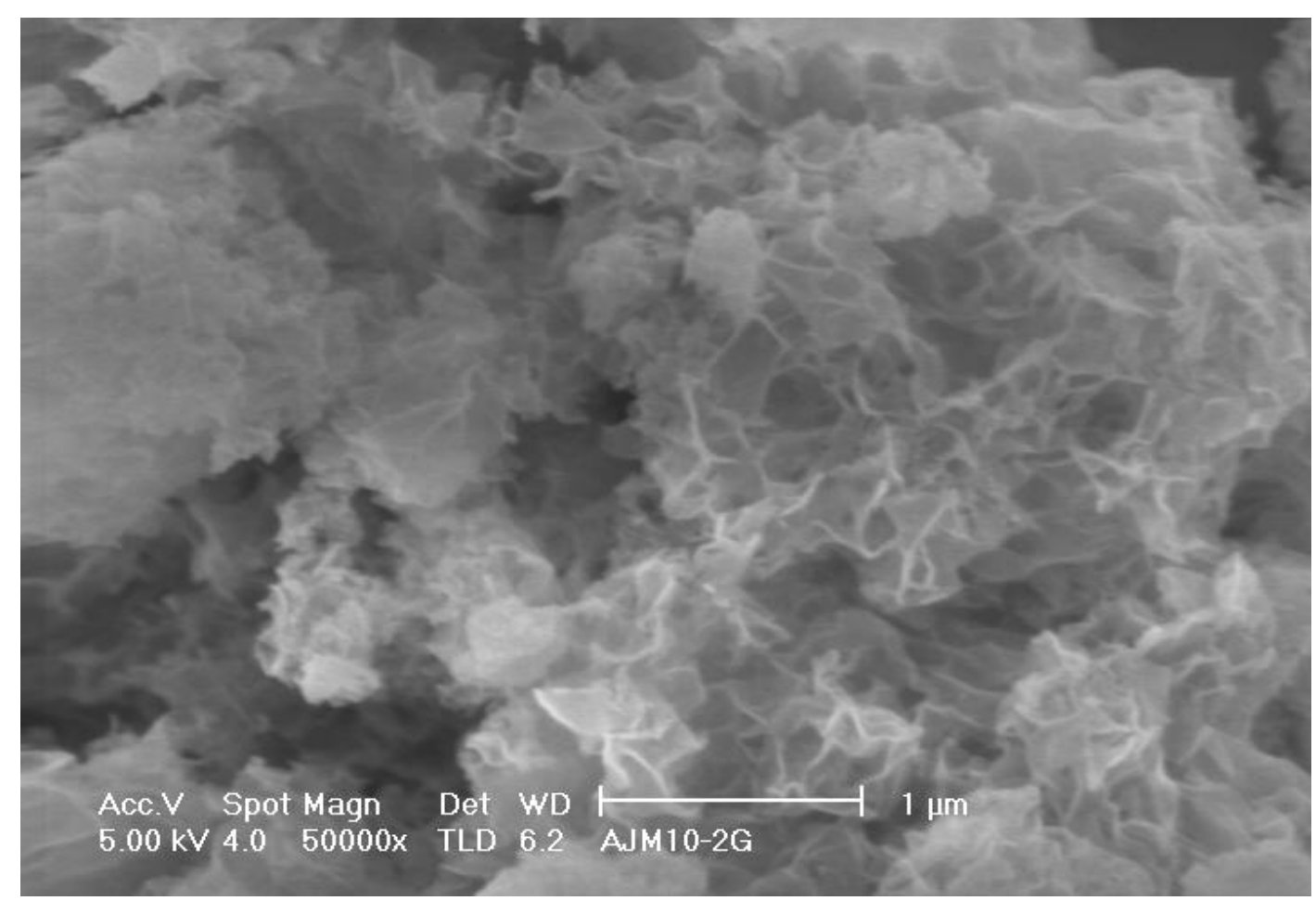

Figure 3.7: Scanning electron micrograph of 2-ethoxyethanol treated nano-structured calcium silicate 300 minutes after precipitation.

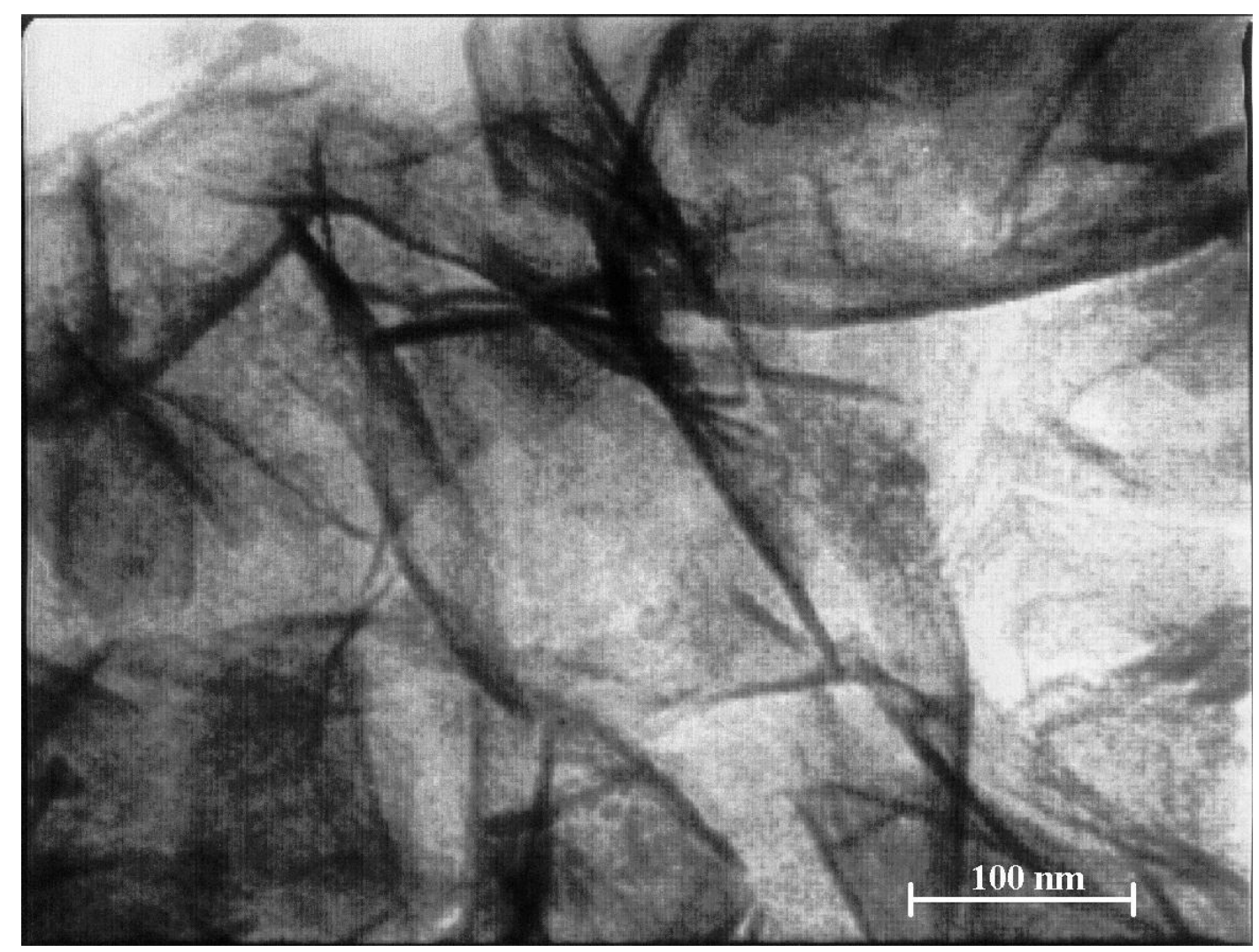

Figure 3.8: A transmission electron micrograph of a fully aged nano-structured calcium silicate that has been treated with 2-ethoxyethanol. 
As would be expected, the development of this open and porous structure is mirrored by the change in the physical properties of the material over this time (Figure 3.9). Due to the bonding between the material's surface and water as a result of surface tension, the porous structure of the material collapses as water is removed from the pores during drying. As detailed in Chapter 4 various methods can be used to keep the open framework such as treating the material with 2-ethoxy- or 2-methoxyethanol. When the collapse of the structure is prevented, both the oil absorption capacity and surface area of the material improve significantly during the ageing process until reaching a plateau when the structure is fully developed.

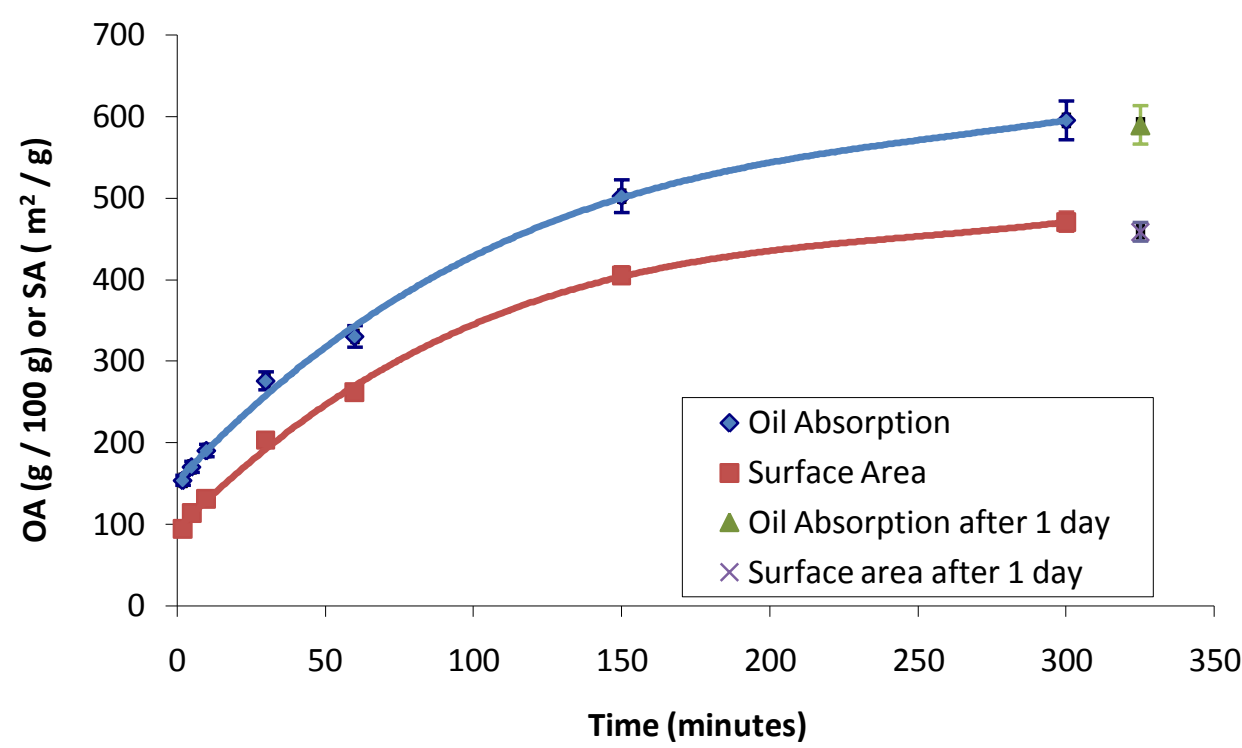

Figure 3.9: Change in physical properties during the ageing process of materials that have been treated with 2-ethoxyethanol to prevent collapse of the structure.

The result of the increase in specific surface area is shown in Figure 3.10 whereby the change in weight lost on ignition (LOI) at $100{ }^{\circ} \mathrm{C}$ is measured for samples taken at different times during the ageing of the material. The weight lost is due chiefly to the loss of chemically bound water, water from the condensation of surface silanol groups, and bound 2-methoxyethanol for the samples treated with this solvent. The loss on ignition is given as a percentage $(\mathrm{w} / \mathrm{w})$ based upon the weight of the sample when dried at $110^{\circ} \mathrm{C}$. The increase in weight lost from the samples taken progressively during the ageing process is likely to be due to the increase of the surface area, which also becomes increasingly accessible, and can therefore accommodate more of these bound 
groups. The sample washed only with water, and therefore has a structure that collapsed upon drying, has a similar \% LOI to the material washed with 2methoxyethanol after only being aged for 30 minutes and is consequently only beginning to develop its porous structure.

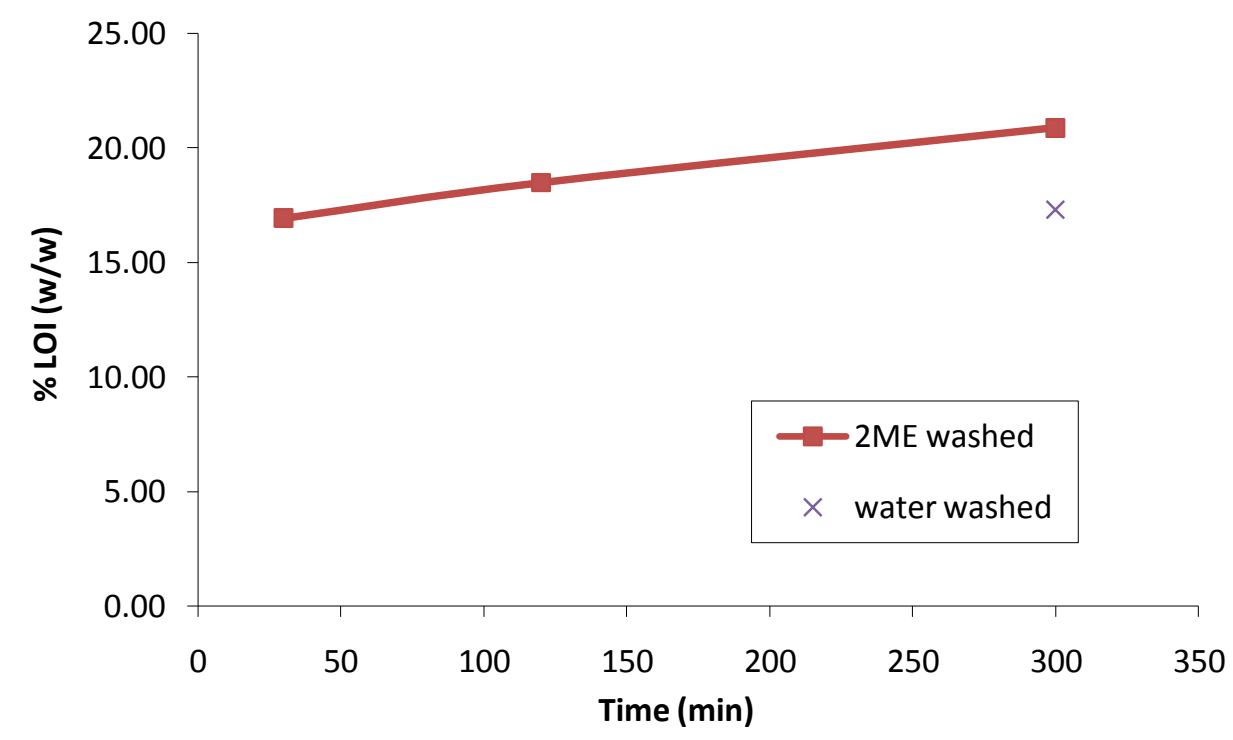

Figure 3.10: Loss of volatile materials from samples taken during ageing when ignitited at 1000 ${ }^{\circ} \mathrm{C}$.

This substantial transformation to the structure of the particles is an internal process as both SEM images and particle size analysis show no corresponding change in either the size or shape of the particles (Figure 3.11). The particle size, measured using laser scattering, is often greater than that observed with electron microscopy and may therefore be influenced by some degree of aggregation and the true particle size not observed. A trend towards a smaller measured particle size was observed during the ageing process, especially during the first 5 minutes. This is likely to be due to the breaking up of aggregates formed during the precipitation as a result of the extended period of stirring throughout this process. 

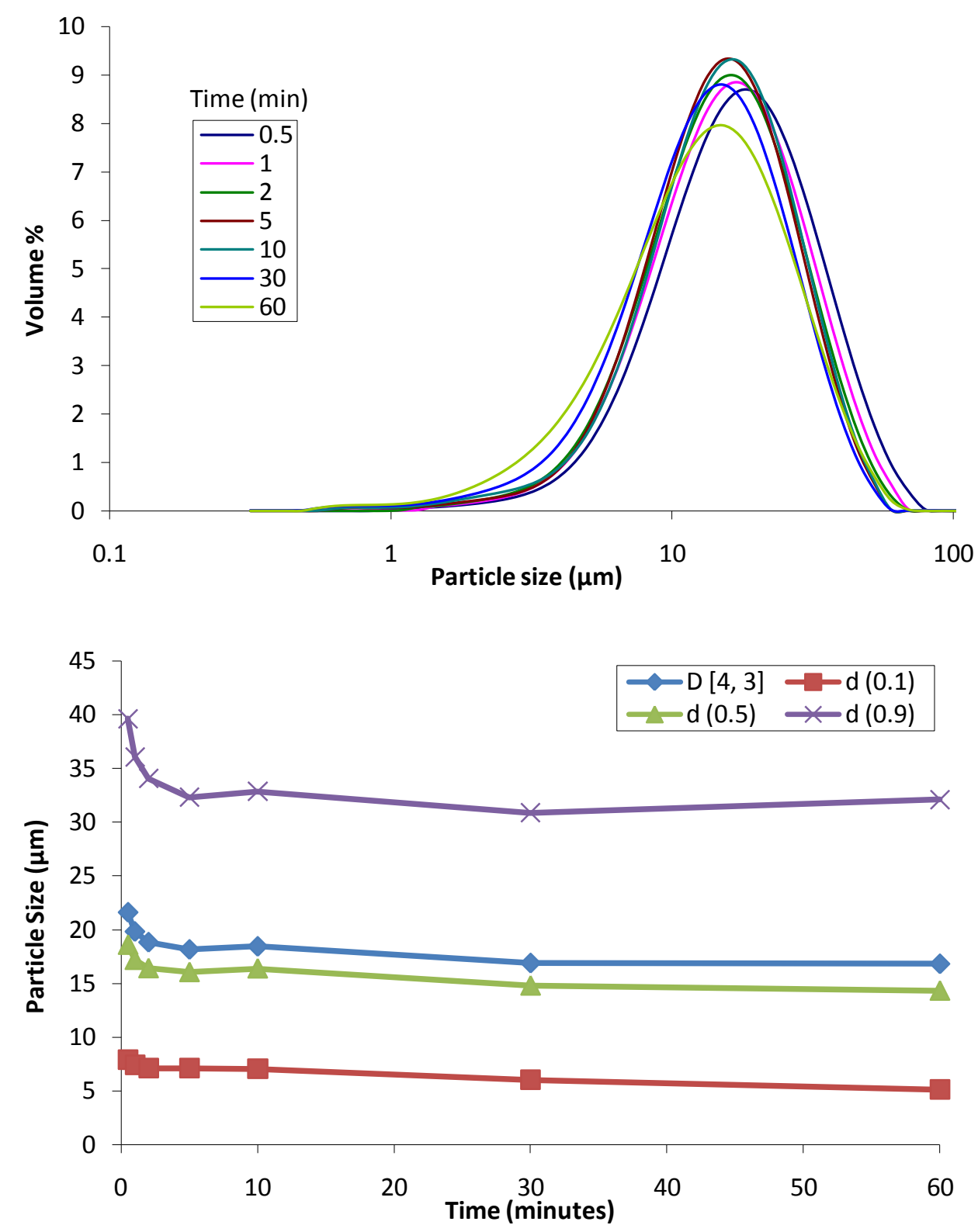

Figure 3.11: Particle size changes over time during the ageing process. Distribution curves by volume $\%$ (top). Volume weighted mean particle size, D[4,3]; and the particle size that $10 \%$ $d(0.1), 50 \% d(0.5)$, and $90 \% d(0.9)$ of the distribution is under (bottom).

The self-assembly of the desert-rose structure during the ageing process is presumably a reorganisation from the kinetic product formed during the rapid precipitation to the more thermodynamically favoured product. It has been observed with ${ }^{29} \mathrm{Si}$ NMR spectroscopy that during the development of the structure in the ageing process, the material becomes increasingly ordered on an atomic level, as evidenced by the narrowing of the main peak at c.a. -86 ppm in Figure 3.12. Guidelines have been overlaid onto Figure 3.12 as an indication of the chemical shifts for the different chemical environments $\left(Q^{0}\right.$ to $\left.Q^{5}\right)$. These values are from the study of related materials, some crystalline calcium silicate 
hydrates (Cong \& Kirkpatrick, 1996) and the highly disordered calcium silicate hydrate (C-S-H) (Cong \& Kirkpatrick, 1996a). The largest peak of the spectra ($86 \mathrm{ppm}$ ) indicates that the material is dominated by $\mathrm{Q}^{2}$ sites as found in pyroxenes, examples of which being wollastonite $\left(\mathrm{CaSiO}_{3}\right)$ and $1.4 \mathrm{~nm}$ tobermorite $\left(\mathrm{Ca}_{5} \mathrm{Si}_{6} \mathrm{O}_{16}(\mathrm{OH})_{2 .} \cdot \mathrm{H}_{2} \mathrm{O}\right)$, with this connectivity being due to the structure consisting of polysilicate chains.

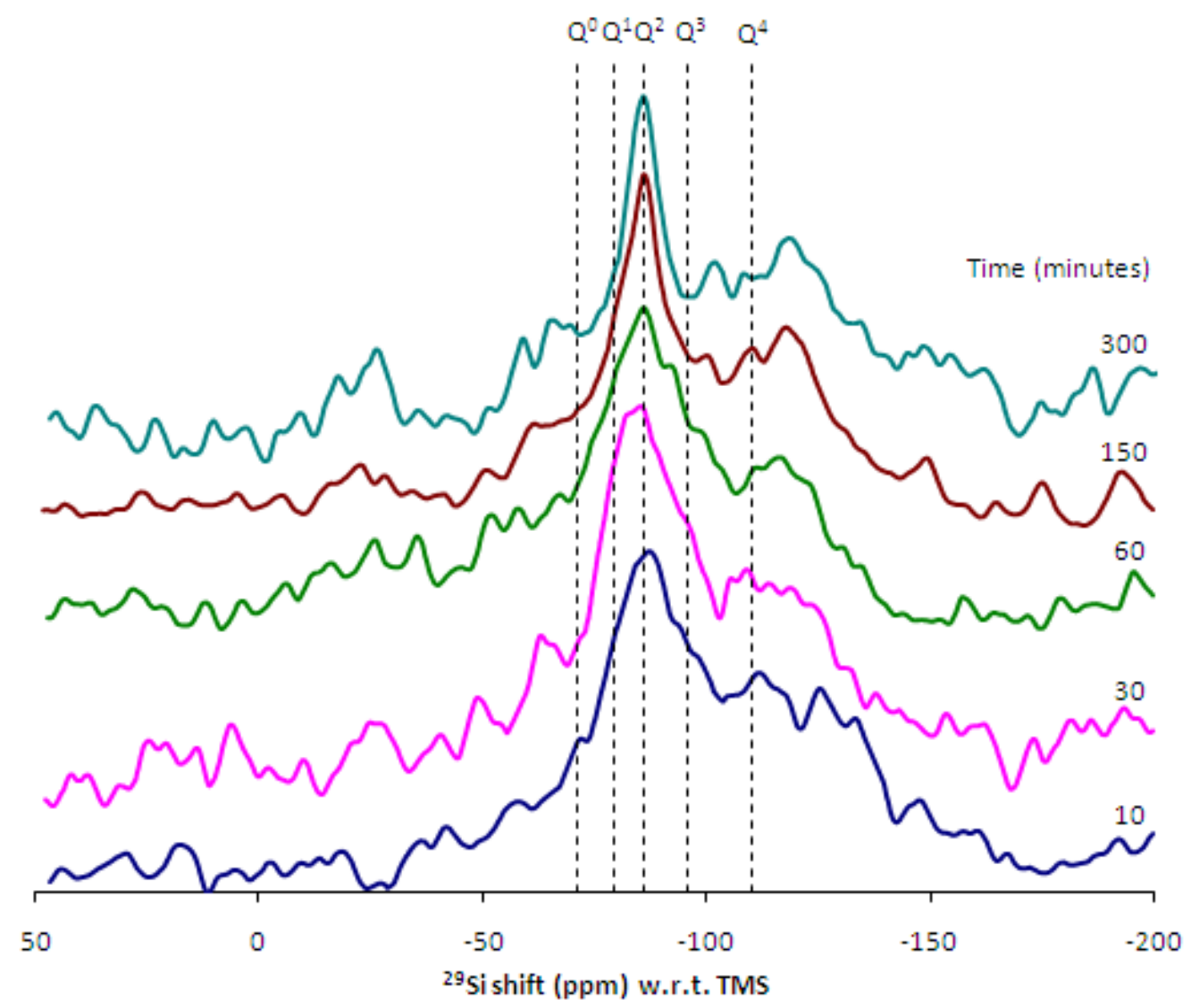

Figure 3.12: ${ }^{29} \mathrm{Si}$ NMR spectra indicating the change in the atomic environment during the ageing process.

There is no peak at -71 ppm $\left(\mathrm{Q}^{0}\right)$ as would be found for the presence of unreacted monomers and the absence of a peak at -79.5 ppm $\left(\mathrm{Q}^{1}\right)$ indicates the absence of both dimers and large numbers of end chain members, which suggests that the chains have significant length. The absence of peaks at -95.7 ppm $\left(Q^{3}\right)$ and $-110 \mathrm{ppm}\left(\mathrm{Q}^{4}\right)$ confirms that there is no branching of the polysilicate chains and no detectable amount of the monomeric silica has polymerised to form a silica. 
As well as the increasing order on an atomic scale, the changes in the X-ray diffraction patterns of the material during the ageing process indicate an increasing degree of order in the long-range (Figure 3.13).

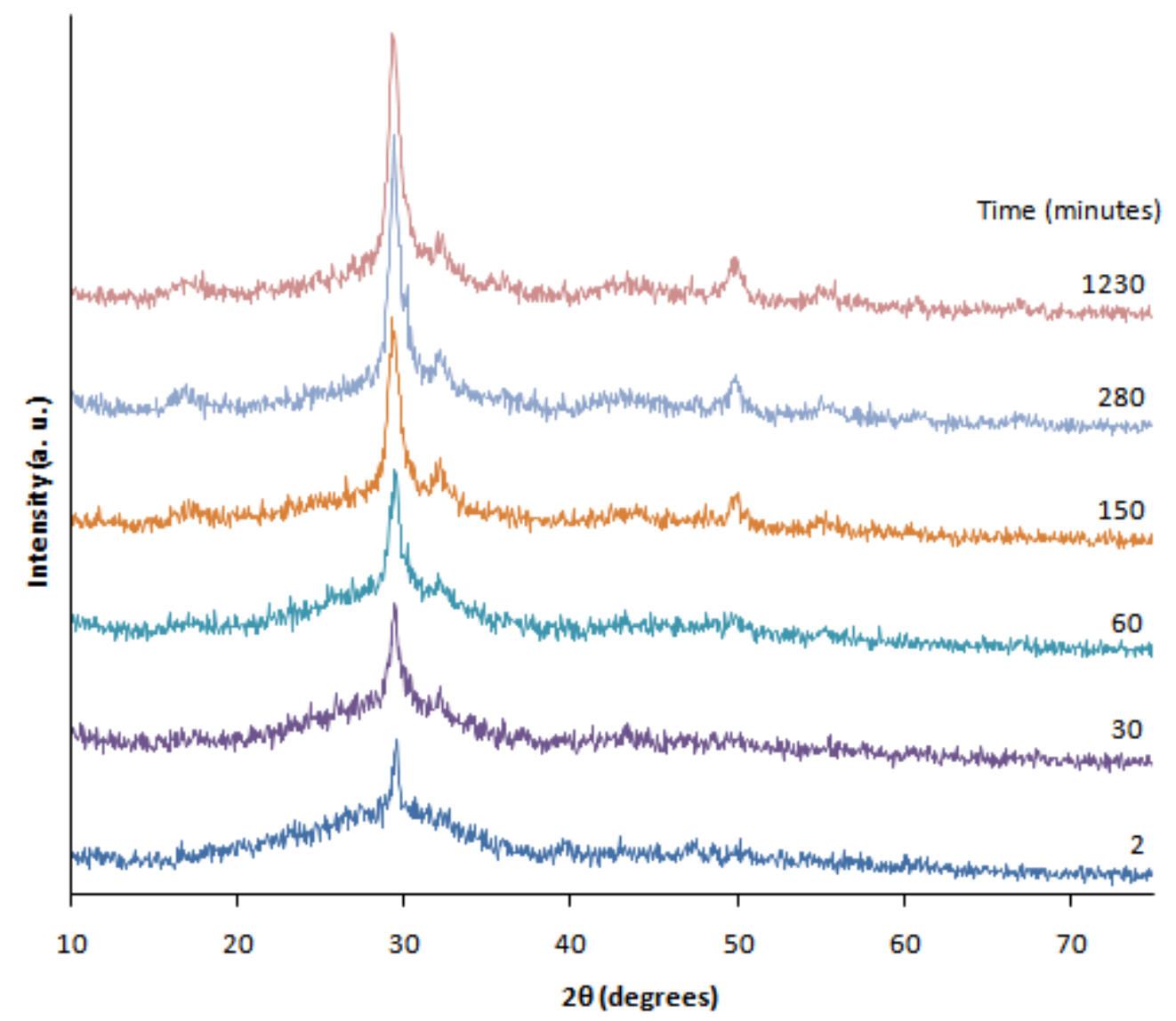

Figure 3.13: X-ray diffractograms of nano-structured calcium silicate during ageing.

The very broad hump between $2 \theta$ of 20 to $35^{\circ}$, due to the more highly disordered nature of the material directly after precipitation, becomes reduced as other broad peaks at $29.3,32.2$, and $49.9^{\circ}$ increase as the ageing process proceeds. These broad peaks are indicative of a poorly crystalline material and are similar those observed for calcium silicate hydrates previously studied such as in the work of Chen et al. (2004) on hydrated calcium silicates with varying $\mathrm{Ca} / \mathrm{Si}$ ratios (Figure 3.14). Spectra containing peaks with these spacings have been used to distinguish near amorphous tobermorite-like phases from other calcium silicate hydrates (Taylor, 1964), an example of which being powder diffraction file (PDF) 34-0002. 


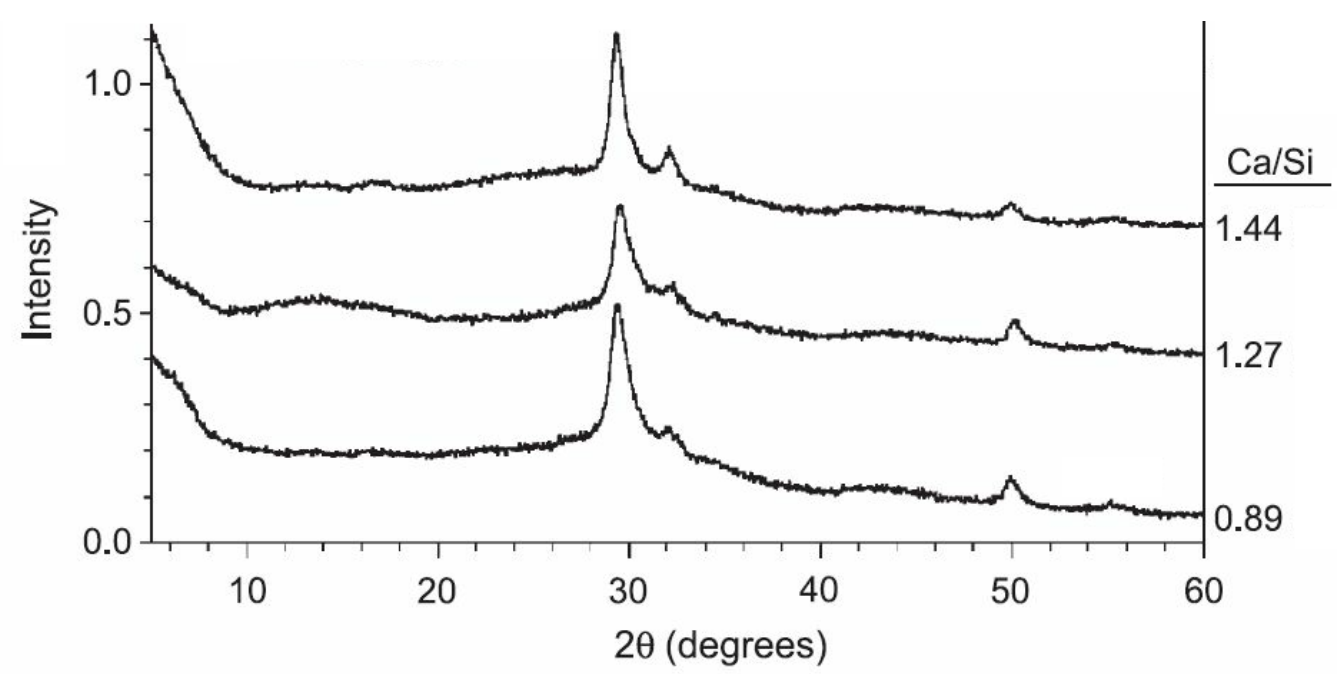

Figure 3.14: X-ray diffractograms of calcium silicate hydrates with varying compositions. (Chen et al., 2004)

More than 30 synthetic and natural crystalline calcium silicate hydrates are known (Shaw et al., 2000), with preparations made at room temperature possessing complex structures ranging from semi-crystalline to nearly amorphous. These materials are extensively studied as hydrated calcium silicate gel is one of the main products of the binding process in Portland cement and is responsible for the setting and hardening actions (Taylor, 1993). These gels are often denoted as "C-S-H" as these materials vary in composition of $\mathrm{CaO}, \mathrm{SiO}_{2}$, and $\mathrm{H}_{2} \mathrm{O}$ and form as a result of the hydration of tricalcium silicate $\left(\mathrm{C}_{3} \mathrm{~S}\right)$ or $\beta$-dicalcium silicate $\left(\beta-\mathrm{C}_{2} \mathrm{~S}\right)$ which together constitute ca. $75 \%$ of a Portland cement by weight (Chen et al., 2004). Due to the great degree of disorder in C-S-H gels, a limited amount of structural information has been obtained by direct study of the materials themselves. However, the C-S-H gel phase has been found to be related to the crystalline minerals tobermorite and jennite about which more detail is known, although these minerals themselves also possess disorder in their structures (Taylor, 1993; Yu et al., 1999; Manzano et al., 2007). Materials similar to C-S-H gels have also previously been synthesised by the action of water on tricalcium silicate, the double replacement reaction of calcium nitrate and sodium silicate, or the reaction of calcium hydroxide with a silica gel (Steinour, 1947; Taylor, 1950, 1953, 1993; Fujii \& Kondo, 1981; Chen et al, 2004). The Ca/Si ratio of these synthetic C-S-H materials can be subsequently varied by decalcification, through the use of concentrated ammonium nitrate solutions. The synthetic C-S-H materials were 
found to have greater structural order than the C-S-H gels but less order than the crystalline minerals. The synthetic C-S-H's also possess a wider range of composition when compared with the crystalline minerals, much like C-S-H gel. The lack of order in the structures allows for the accommodation of a high number of defects that enable the $\mathrm{Ca}$ /Si ratio to vary from 0.67 to 1.5 (Chen et al, 2004). The X-ray diffraction patterns of these materials (Taylor, 1950; Fujii \& Kondo, 1981; Chen et al, 2004) are similar to that of the nano-structured calcium silicate of this work although none are known to possess the same porous macro-structure. The similarity of these patterns suggests that nanostructured calcium silicate is also structurally related to tobermorite to some degree.

The crystal structure of $1.4 \mathrm{~nm}$ tobermorite (also known as plombierite, $\left.\mathrm{Ca}_{5} \mathrm{Si}_{6} \mathrm{O}_{16}(\mathrm{OH})_{2} .7 \mathrm{H}_{2} \mathrm{O}\right)$ has only recently been solved by the use of orderdisorder (OD) theory by Bonaccorsi \& Merlino (2005). This followed earlier studies by Hamid (1981) to solve $1.1 \mathrm{~nm}$ tobermorite as well as the determination of the real structures of $0.9 \mathrm{~nm}, 1.1 \mathrm{~nm}$ tobermorites, and clinotobermorite using OD theory (Merlino et al., 1999; Merlino et al. 2000; Merlino et al, 2001) . The structure is built up of complex layers, formed by sheets of sevenfold coordinated calcium cations capped with water molecules and flanked on both sides by wollastonite-like chains (Figure 3.15).

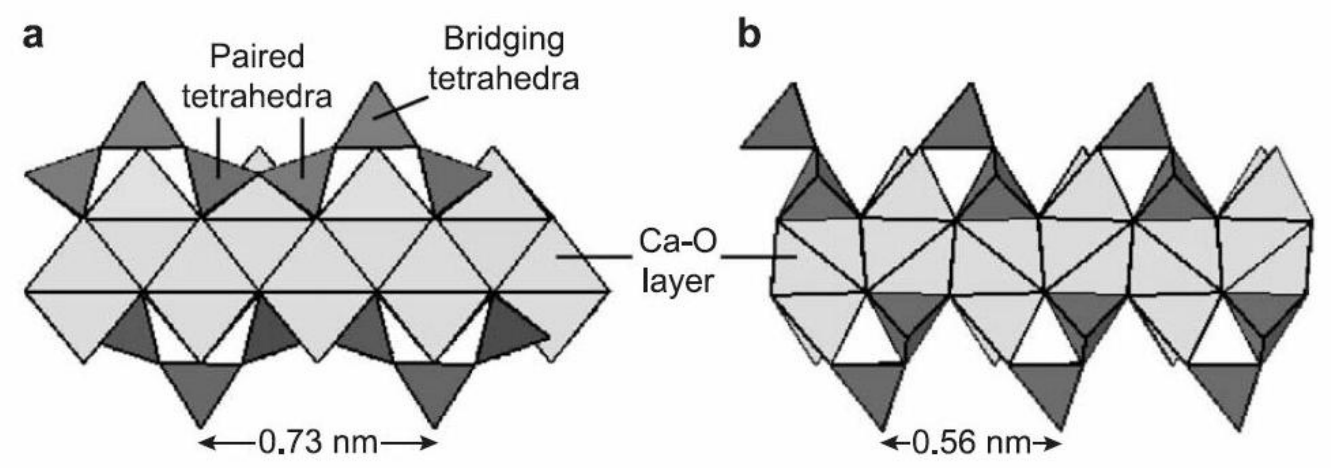

Figure 3.15: Single layer of $1.4 \mathrm{~nm}$ tobermorite seen along (a) [210] and (b) [010] illustrating the Ca-O main layer with attached silicate tetrahedra dreierketten chains. (Chen et al., 2004) 
The wollastonite-like chains are made up of three tetrahedra repeating units (dreierketten) consisting of paired tetrahedra bridged by another unit. These layers can align themselves in two possible ways (Figure 3.16), thereby causing long range disorder along the $\mathrm{c}$ axis.

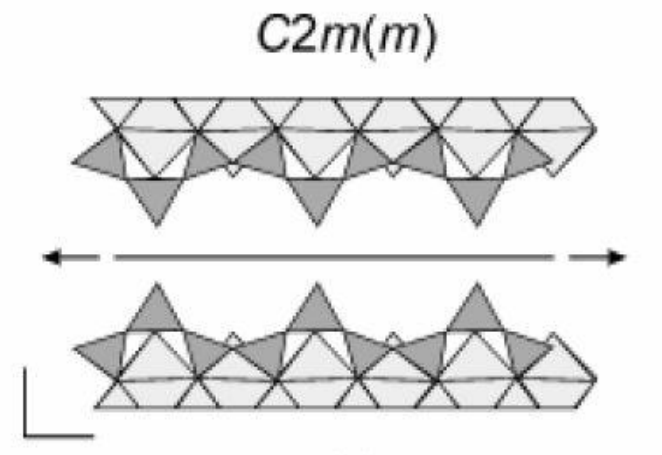

(a)

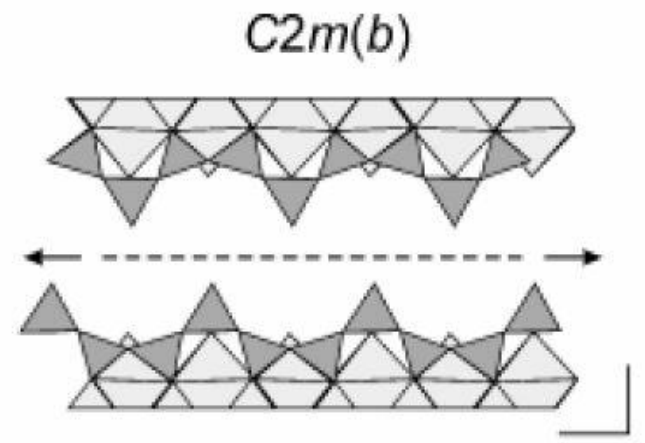

(b)

Figure 3.16: The two possible layers of $1.4 \mathrm{~nm}$ tobermorite, and their symmetry elements. (Bonaccorsi et al, 2005)

The $1.4 \mathrm{~nm}$ spacing between the layers parallel to (001) is occupied with water molecules and calcium ions. The interlayer calcium ions share two oxygen atoms with bridging tetrahedra while additional bonds to water molecules complete the octahedral co-ordination (Figure 3.17). The ability of these sites to be equally occupied by either of these species causes their random distribution between the sites and causes variation in the $\mathrm{Ca} / \mathrm{Si}$ ratio of the material. The highly disordered gel and synthetic C-S-H also allow the $\mathrm{Ca} / \mathrm{Si}$ ratio to increase by a greater degree through the omission of bridging tetrahedra and/or increasing the interlayer calcium by loss of protons from $\mathrm{Si}$ $\mathrm{OH}$ groups. C-S-H gels can also consist of a mix of both tobermorite- and the

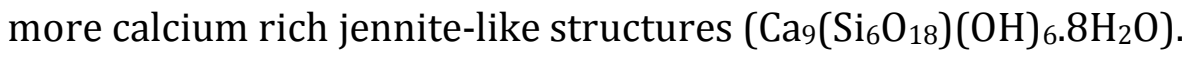




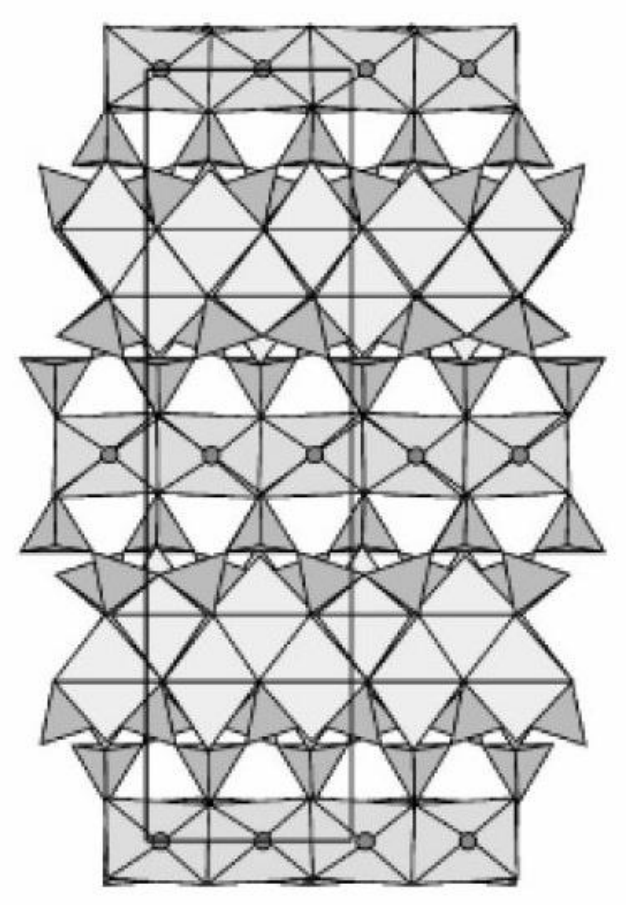

(a)

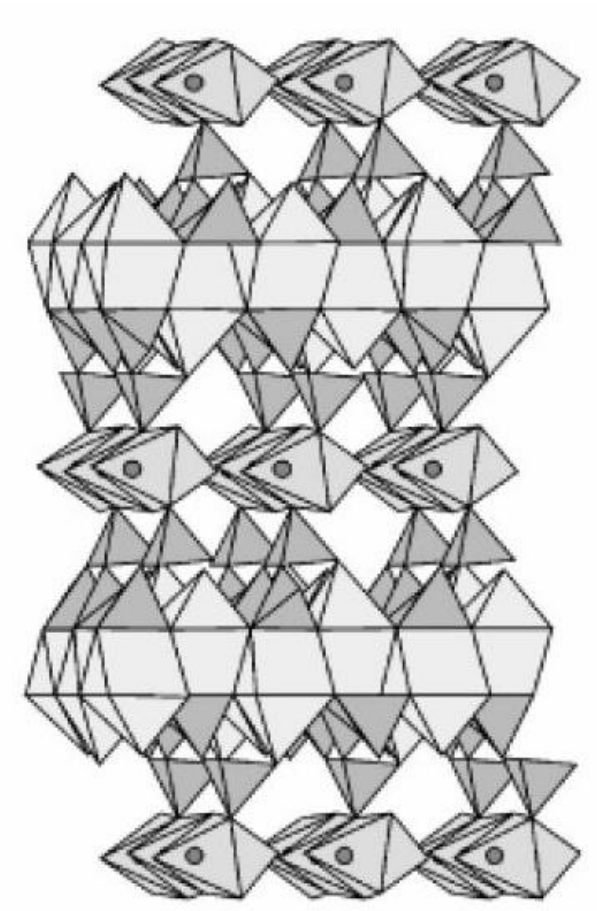

(b)

Figure 3.17: Crystal structure of $1.4 \mathrm{~nm}$ tobermorite (a) perpendicular to (100) and (b) approximately down [010]. Interlayer octahedral calcium are shown (also in light grey) with dark grey circles that represent water molecules that have an equal probability to occupy the sites instead. (Bonaccorsi et al., 2005)

Upon heating $1.4 \mathrm{~nm}$ tobermorite at 80 to $105^{\circ} \mathrm{C}$, the interlayer spacing collapses which results in the formation of $1.1 \mathrm{~nm}$ tobermorite (that has a composition ranging from $\mathrm{Ca}_{5} \mathrm{Si}_{6} \mathrm{O}_{17} .5 \mathrm{H}_{2} \mathrm{O}$ to $\mathrm{Ca}_{4} \mathrm{Si}_{6} \mathrm{O}_{15}(\mathrm{OH})_{2} .5 \mathrm{H}_{2} \mathrm{O}$ ) as some water is removed from the cavity (Bonaccorsi \& Merlino., 2005). While the wollastonite-like chains of each layer in 1.4-nm tobermorite are separated, some forms of naturally occurring $1.1 \mathrm{~nm}$ tobermorite possess double chains by the sharing of an oxygen atom by the bridging tetrahedra. Bonaccorsi \& Merino (2005) noted that upon the dehydration of $1.4 \mathrm{~nm}$ tobermorite to form $1.1 \mathrm{~nm}$ tobermorite, the single silicate chains were maintained. There is no evidence from the ${ }^{29} \mathrm{Si}$ NMR of nano-structured calcium silicate of any presence of $\mathrm{Q}^{3}$ sites in Figure 3.12 as would be expected for the formation of double chains between layers correctly aligned (with $\mathrm{C} 2 \mathrm{~m}(\mathrm{~m})$ symmetry - Figure 3.16a). As was shown in Figure 3.11 the majority of silicon within nano-structured calcium silicate is present as single silicate chains $\left(Q^{2}\right)$ which is in agreement with that observed for tobermorite. Additional dehydration of $1.4 \mathrm{~nm}$ tobermorite at $300^{\circ} \mathrm{C}$ causes further reduction of the interlayer spacing 
resulting in the formation of $0.93-\mathrm{nm}$ tobermorite (also known as riversideite, $\left.\mathrm{Ca}_{5} \mathrm{Si}_{6} \mathrm{O}_{16}(\mathrm{OH})_{2}\right)$. It was observed by Taylor (1953) that heating both natural riversideite and artificially prepared calcium silicate hydrates at $800{ }^{\circ} \mathrm{C}$ resulted in materials that gave X-ray powder patterns identical to that of $\beta$ wollastonite $\left(\mathrm{CaSiO}_{3}\right)$. This same change in the X-ray pattern was observed for the calcining of nano-structured calcium silicate (Figure 3.18).

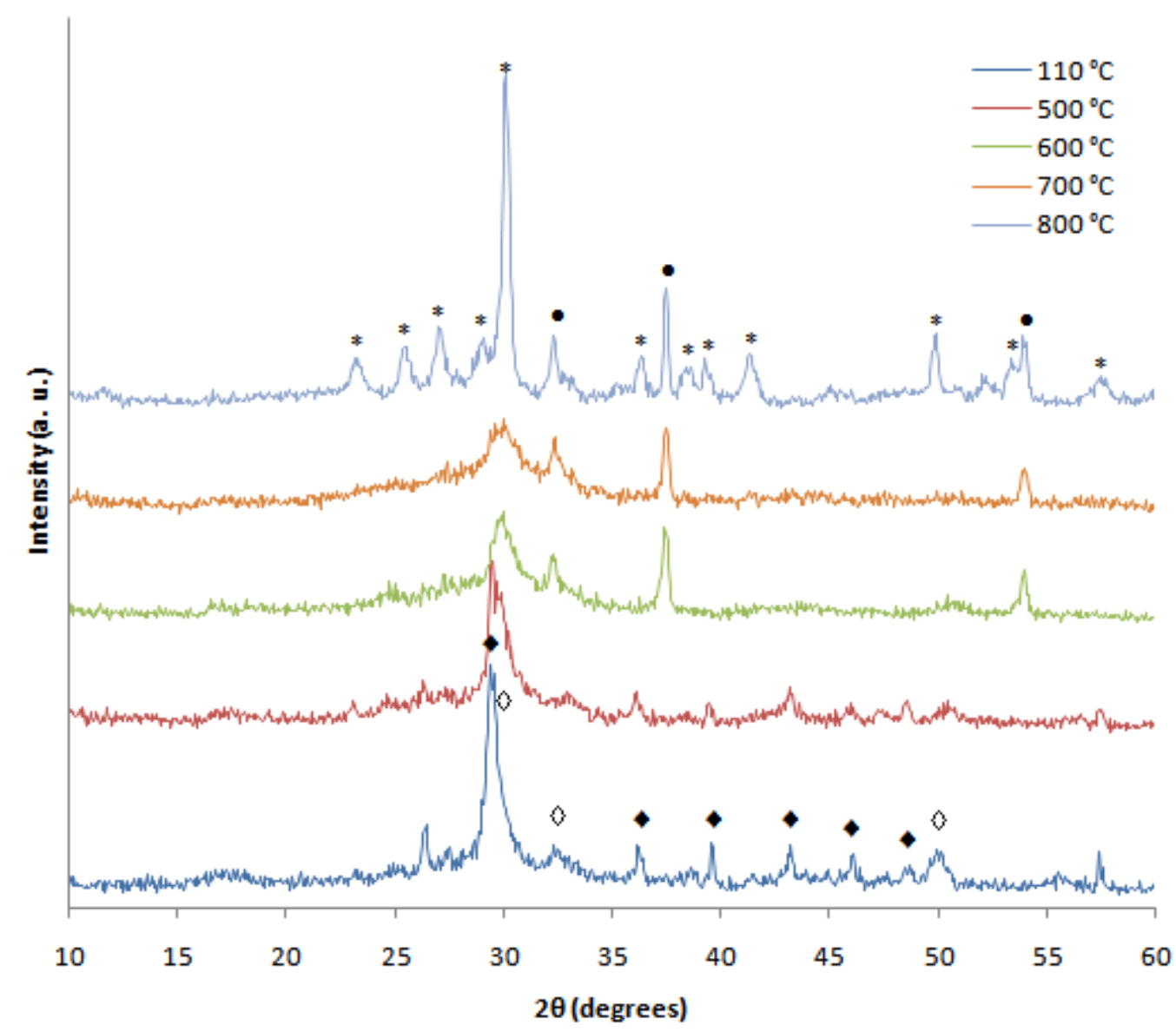

Figure 3.18: X-ray diffractograms of nano-structured calcium silicate that has been calcined at different temperatures $(\diamond=$ hydrated calcium silicate, PDF 34-0002; $\bullet$ calcite, PDF 05-0586; • = calcium oxide, PDF 37-1497; ${ }^{*}=2 \mathrm{M}$ - wollastonite, PDF 27-0088).

When the material is initially dried at $110^{\circ} \mathrm{C}$ the measured pattern contains the broad peaks of disordered hydrated calcium silicate and those of a calcium carbonate impurity, with the most intense peaks of both patterns occurring between 29 to $30^{\circ}$. The calcium carbonate impurity is from the calcium hydroxide used for the reaction and also possibly due to a reaction of the calcium hydroxide with dissolved carbon dioxide over the course of the reaction. Both the hydrated calcium silicate and calcite patterns remain the 
only ones detectable in the material as it was calcined up to $500{ }^{\circ} \mathrm{C}$. At $600{ }^{\circ} \mathrm{C}$ the calcium carbonate has decomposed to calcium oxide so that the most intense peak of the calcium silicate is more clearly visible at ca. $29.4^{\circ}$. The patterns of both the now dehydrated calcium silicate and calcium oxide are also both present at $700{ }^{\circ} \mathrm{C}$. At $800^{\circ} \mathrm{C}$ the pattern of the fully dehydrated monoclinic parawollastonite structured $\beta-\mathrm{CaSiO}_{3}$ is identified as had been observed by Taylor, along with that of calcium oxide from the calcium carbonate impurity.

It was also noted that as well as the atomic structure of the hydrated calcium silicate being observed up to $700{ }^{\circ} \mathrm{C}$, the macro-structure of the material also remained essentially intact when the material was calcined to a temperature of $700{ }^{\circ} \mathrm{C}$. A scanning electron micrograph of the material calcined at $700{ }^{\circ} \mathrm{C}$ shows that the material still consists of nano-sized platelets randomly arranged that bestow the material with its large surface area and pore volume (Figure 3.19). The material calcined at a temperature of $800^{\circ} \mathrm{C}$ no longer possesses any of this fine framework with no platelets visible in the structure (Figure 3.20). As expected, this change in the macro-structure of the material that occurs at the same temperature as the change in the atomic structure, has a significant effect upon the physical properties of the material. Both the oil absorption capacity and the surface area of the material following treatment with 2-ethoxyethanol are stable to a temperature of over $600{ }^{\circ} \mathrm{C}$ before a slight decline at $700{ }^{\circ} \mathrm{C}$ and a rapid reduction in both of these properties from 700 to $800{ }^{\circ} \mathrm{C}$ (Figure 3.21). The stability of the material up to $700{ }^{\circ} \mathrm{C}$ would allow the material to be utilised at elevated temperatures without adverse effects to the structure, which could be of importance for some applications such as if the material was used as a catalyst support. 


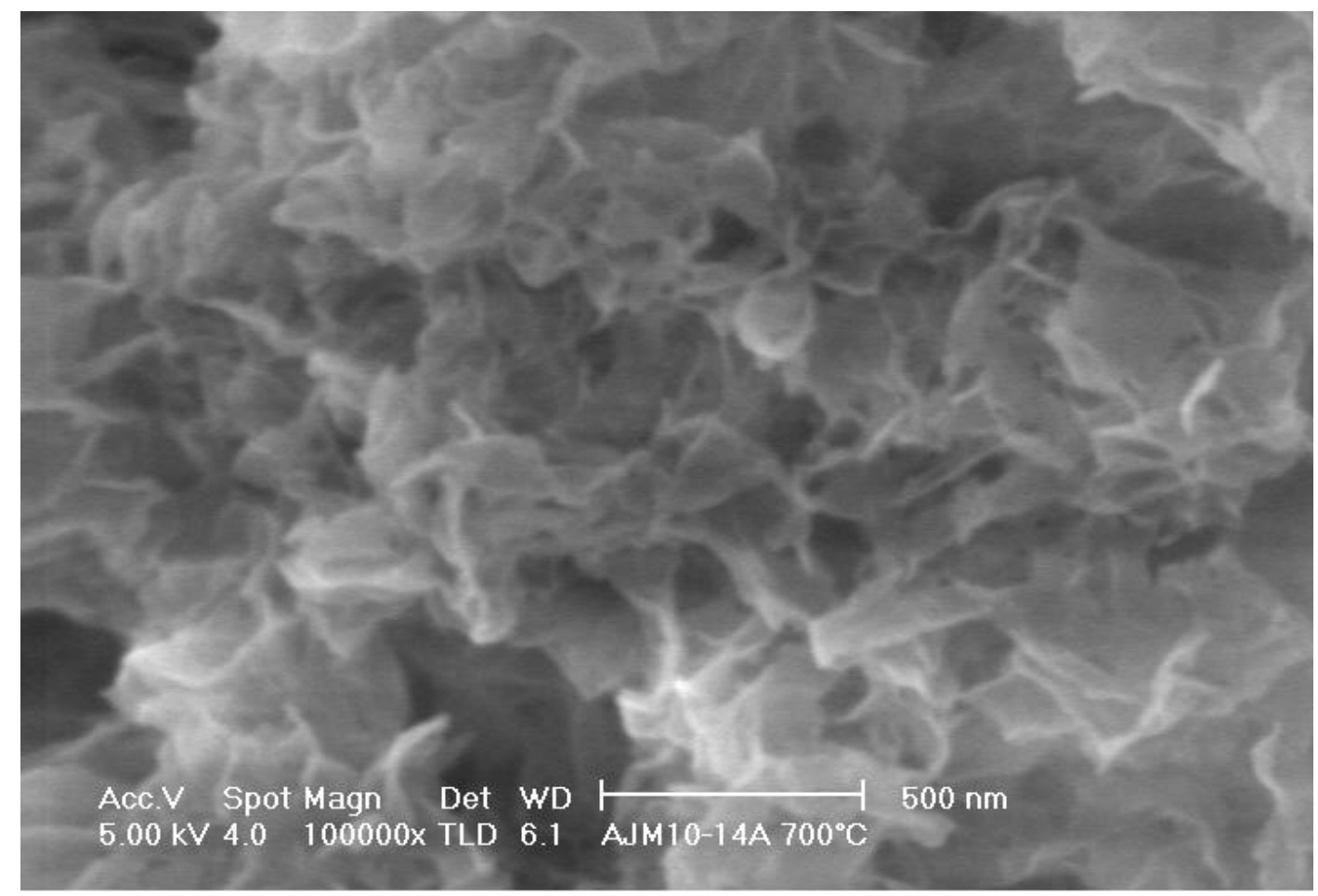

Figure 3.19: Scanning electron micrograph of nano-structured calcium silicate treated with 2ethoxyethanol that has been calcined at $700{ }^{\circ} \mathrm{C}$.

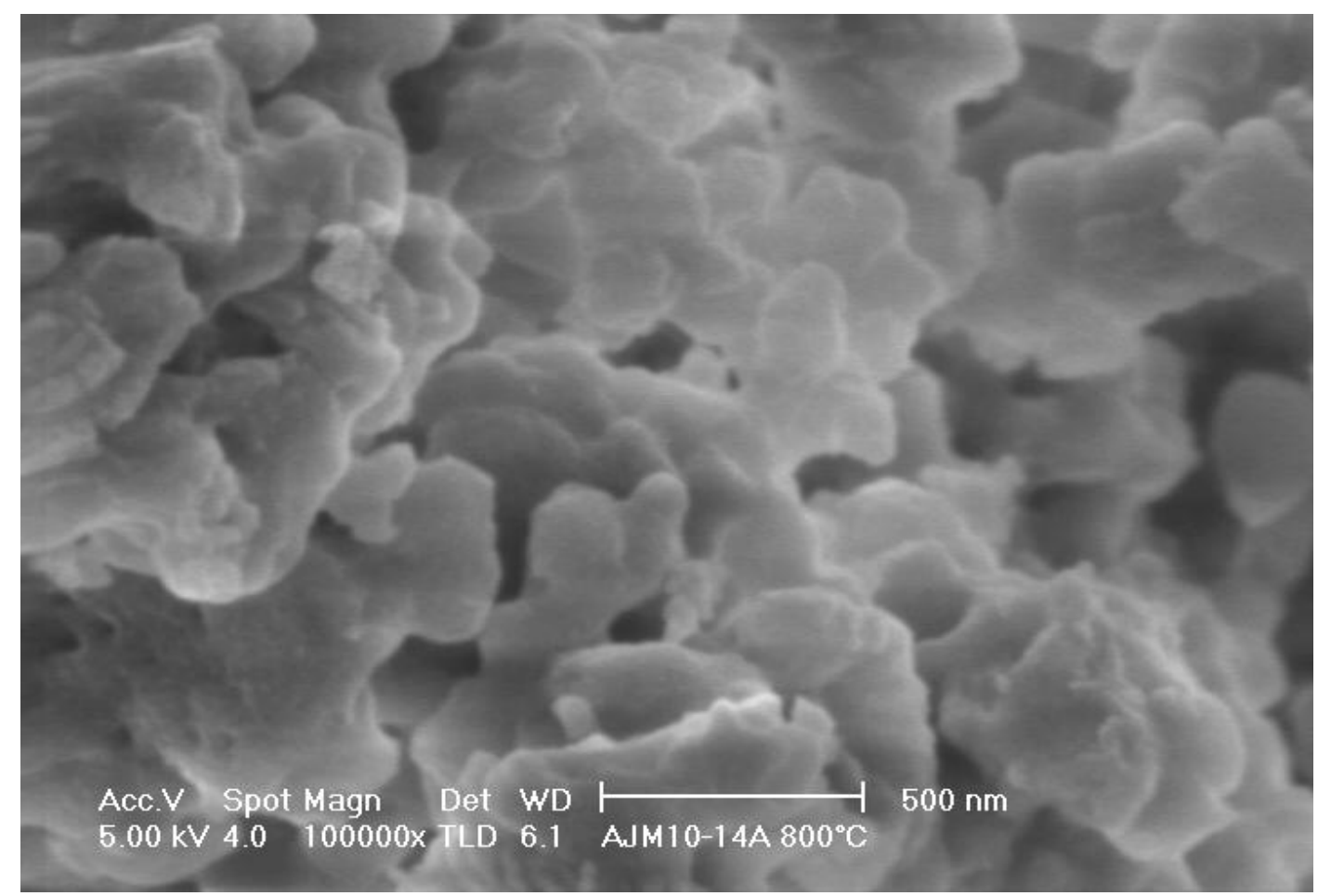

Figure 3.20: Scanning electron micrograph of nano-structured calcium silicate treated with 2ethoxyethanol that has been calcined at $800^{\circ} \mathrm{C}$. 


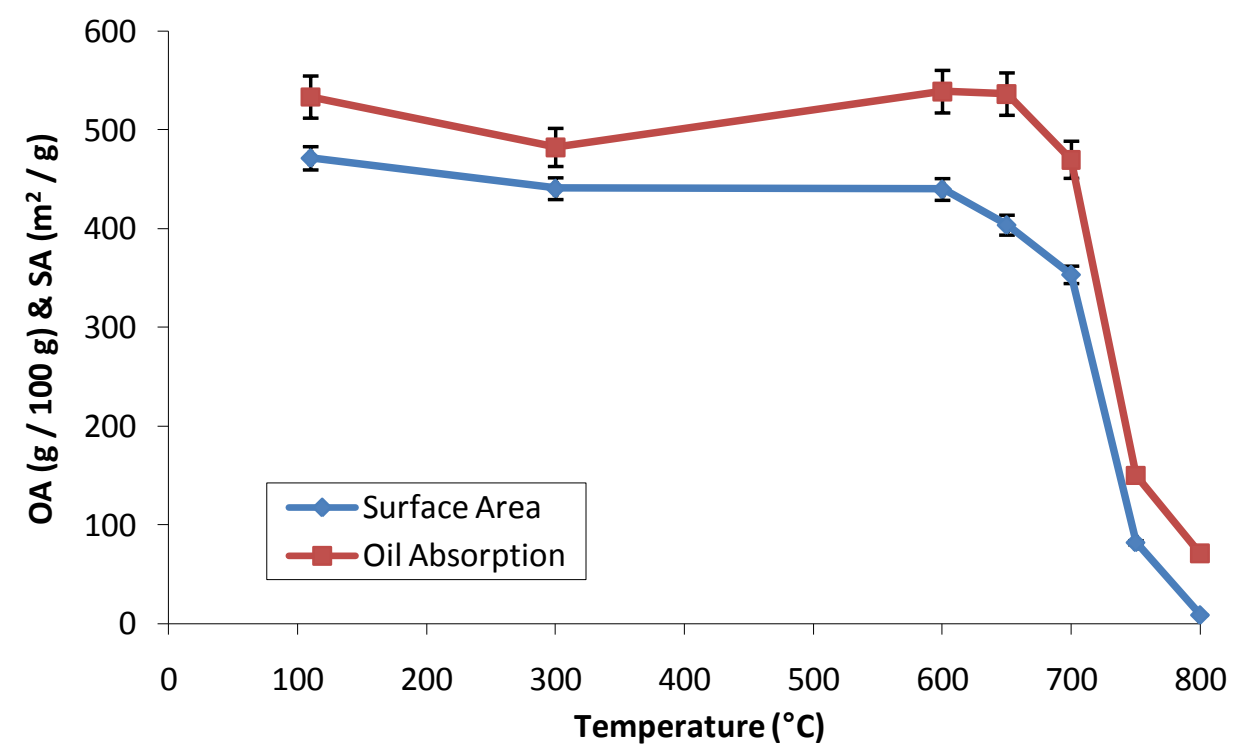

Figure 3.21: Effect of calcining nano-structured calcium silicate treated with 2-ethoxyethanol at different temperatures upon the oil absorption capacity and surface area of the material. 


\section{Chapter 4}

\section{POST-FORMATION TREATMENTS}

A common feature of silica gels and precipitates is the shrinkage of the material during the removal of pore liquid in the drying process. This shrinkage is a result of the structure collapsing due to the surface tension of the water in the pores that can behave as fine capillary tubes. As the structure is essentially inelastic, the collapse of the structure can cause the breaking of inter-particle bonds and likely formation of new bonds at new points of contact, therefore resulting in a denser and less porous material. This issue of structure collapse has been found to also occur with nano-structured calcium silicate. There are five general ways to prevent collapse of the silica gels during removal of the liquid phase (Iler, 1979).

1. Strengthening the gel by reinforcement, increasing the strength of the inter-particle bonds, thus opposing the shrinkage forces.

2. Reducing the surface tension forces by enlarging the pore diameter by an ageing or hydrothermal process.

3. Replacing the water with a polar liquid of lower surface tension.

4. Heating the liquid-filled gel under pressure to above the critical point of the liquid where no liquid-vapour boundary exists and releasing the vapour as is used in the formation of aerogels.

5. Making the surface of the silica hydrophobic.

Some of these techniques have consequently been studied as ways of preventing the collapse of nano-structured calcium silicate. 


\subsection{Application of 2-ethoxyethanol}

It has been found that the replacement of water within the pores of the structure with either 2-ethoxyethanol or 2-methoxyethanol is a very effective means of preventing collapse of the structure upon drying (Figure 4.2). Both of these liquids possess lower surface tensions than water (as given in Table 4.1) and are equally able to prevent structure collapse, although 2-ethoxyethanol was typically preferred for its lower cost.

Table 4.1: Surface tensions at $25^{\circ} \mathrm{C}$ of solvents used for washing samples (Lide, 2005)

\begin{tabular}{lc}
\hline Solvent & Surface tension $\times 10^{-3}(\mathrm{~N} / \mathrm{m})$ \\
\hline Water & 71.99 \\
2-Ethoxyethanol & 28.35 \\
2-Methoxyethanol & 30.84 \\
\hline
\end{tabular}

Other alcohols and polar solvents with low surface tensions have been used without similar success in preventing structure collapse (Borrmann, 2000), and it is thought that the ability of the 2-ethoxyethanol to chelate to accessible surface calcium as shown in Figure 4.1 may have an important role in this function.
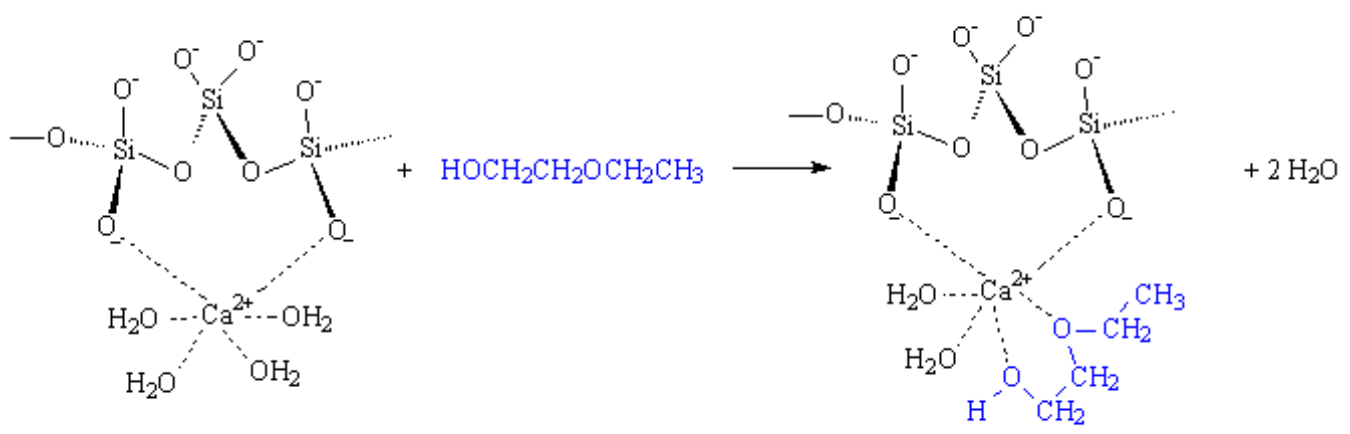

Figure 4.1: A schematic of the chelation of 2-ethoxyethanol to surface calcium groups. 

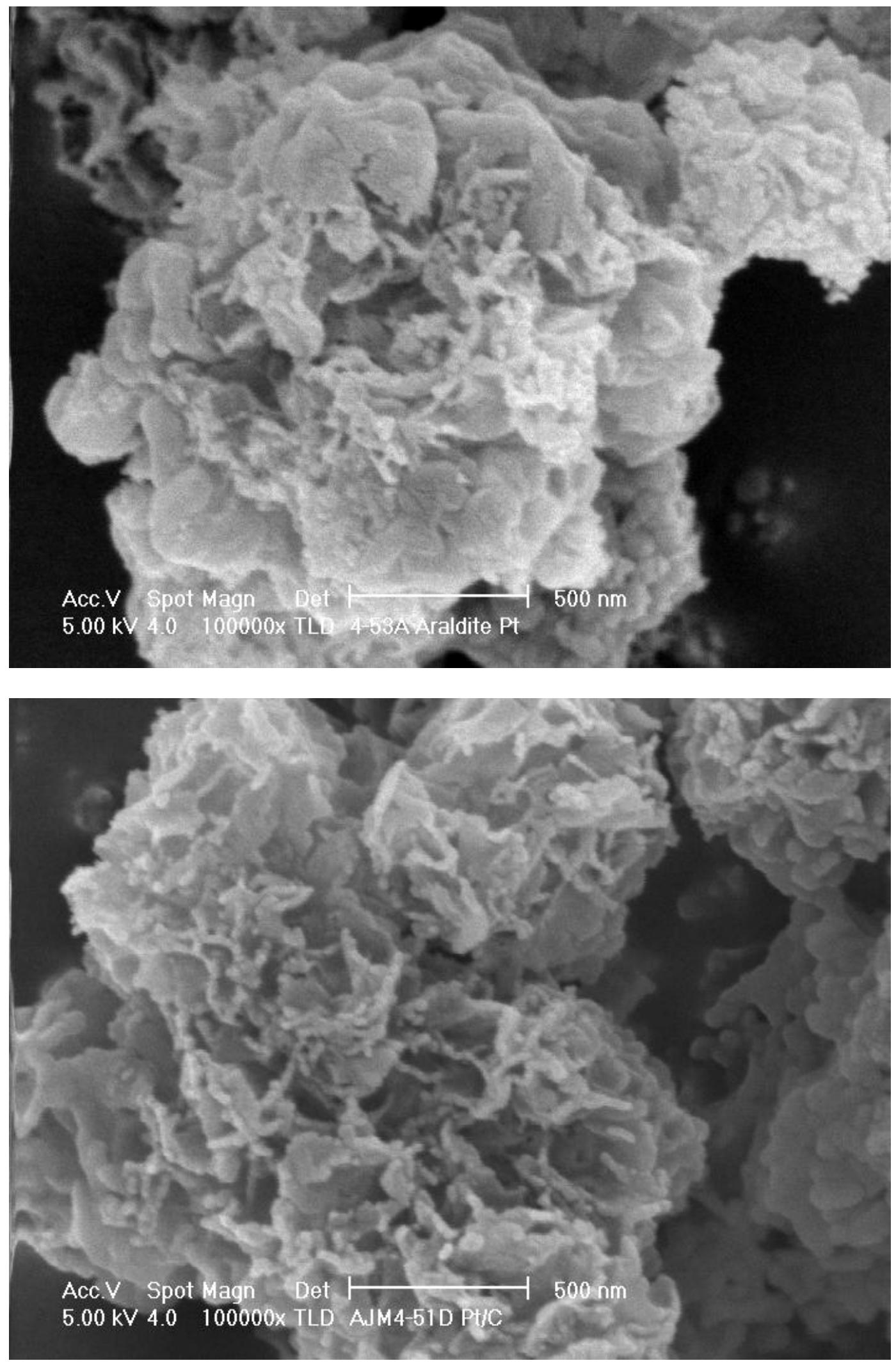

Figure 4.2: Scanning electrons micrographs of a nano-structured calcium silicate that has collapsed upon drying (top) and maintained its structure upon drying at $110{ }^{\circ} \mathrm{C}$ after being treated with 2-ethoxyethanol (bottom). 
The 2-ethoxyethanol was applied as a plug flow wash through the nanostructured calcium silicate filter cake as detailed in section 2.3.2. It was found following experimentation with an aged slurry formed from $0.58 \mathrm{~mol} / \mathrm{L}$ monomeric silica, that a plug flow of 100\% 2-ethoxyethanol approximately equal volume to that of the filter cake once compacted under vacuum was sufficient to maintain a maximum pore volume (Figure 4.3).

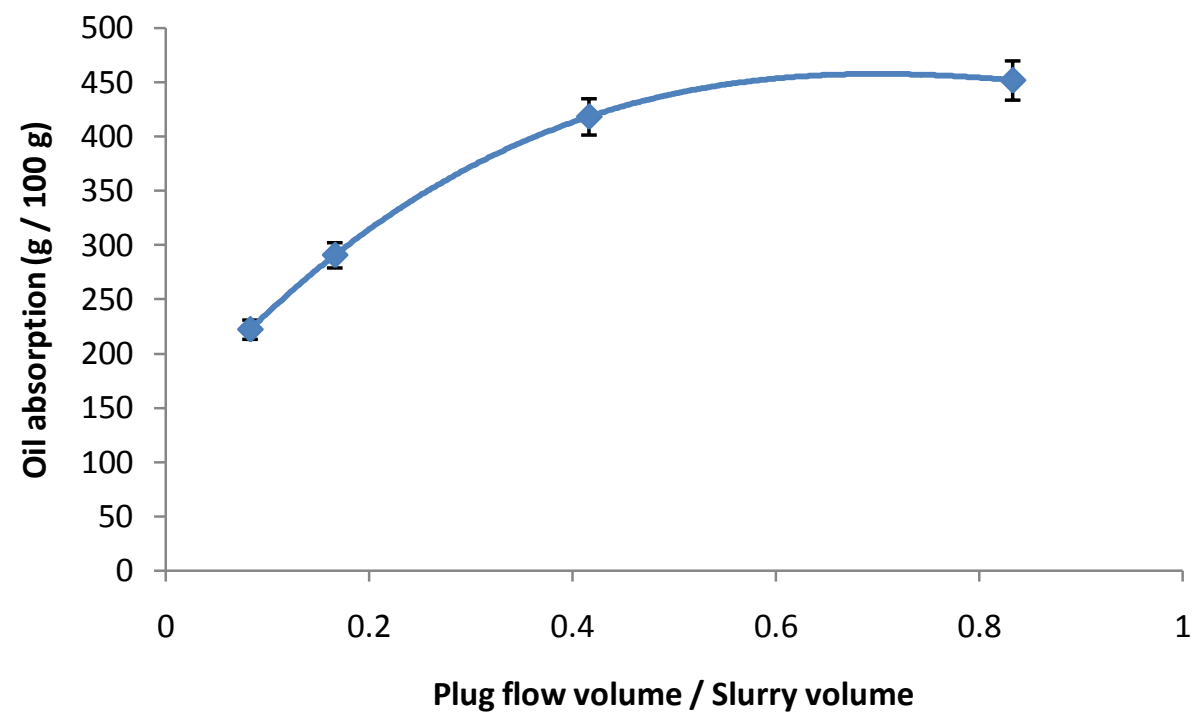

Figure 4.3: The effect of plug flow volume of 2-ethoxyethanol upon the oil absorption capacity.

Studies into the proportion of 2-ethoxyethanol in the plug flow necessary to maximise the pore volume revealed that in excess of $80 \%(\mathrm{v} / \mathrm{v}) 2$ ethoxyethanol to water achieved the best results (Figure 4.4). This influences how the plug flow needs to be applied to the filter cake as the presence of more than $20 \%(\mathrm{v} / \mathrm{v})$ of water from the plug flow of water for washing would have a detrimental effect on the resulting material's oil absorption capacity. The majority of water from the prior plug flow should therefore be allowed to pass through the filter cake before the 2-ethoxyethanol is applied. This also agrees with the volume of the plug flow necessary, as all of the water present in the pores of the filter cake needs to be replaced for structure collapse to be avoided.

As well as the ability to prevent the collapse of the structure, the replacement of water in the pores by 2-ethoxyethanol prior to drying at $110^{\circ} \mathrm{C}$ also influences 
the weight of material produced (Figure 4.5). The increase in weight as the proportion of 2-ethoxyethanol in the plug flow increases is probably due to both the replacement on the surface for a group with a higher molecular weight as well as the increase in surface area allowing for a larger number of groups to bind to the surface.

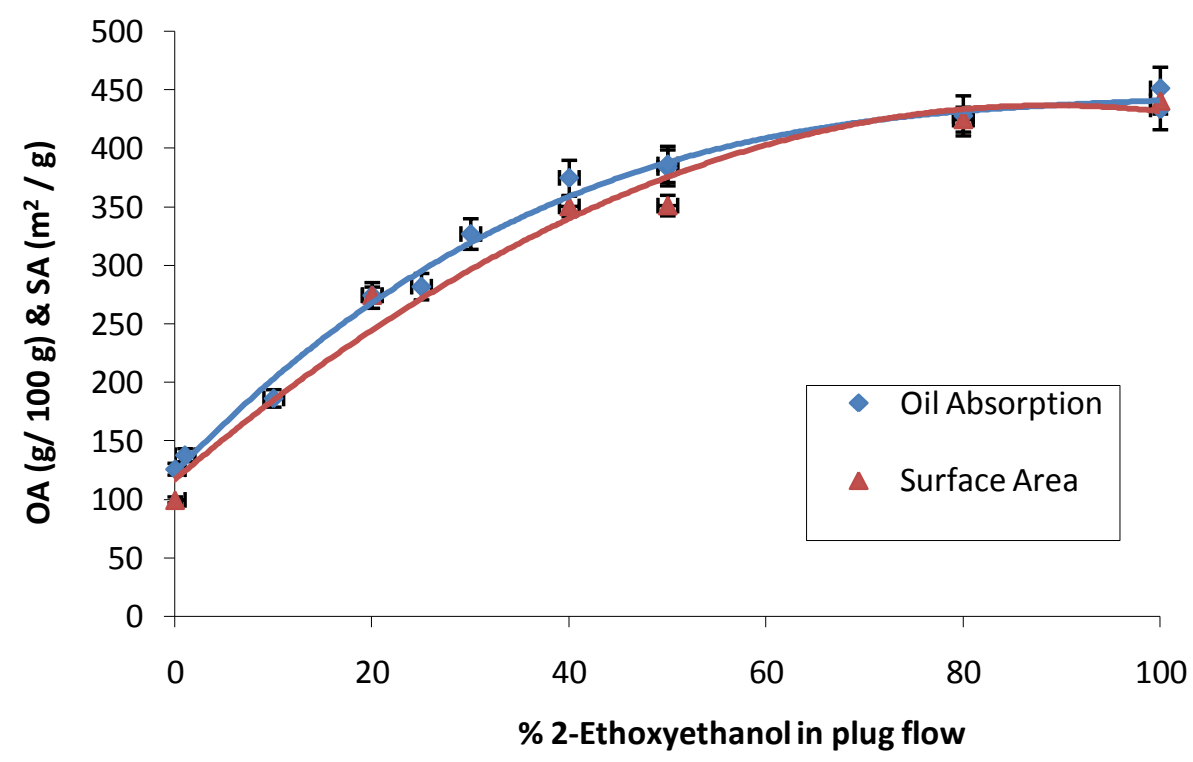

Figure 4.4: Effect of the proportion of 2-ethoxyethanol in plug flow upon the physical properties.

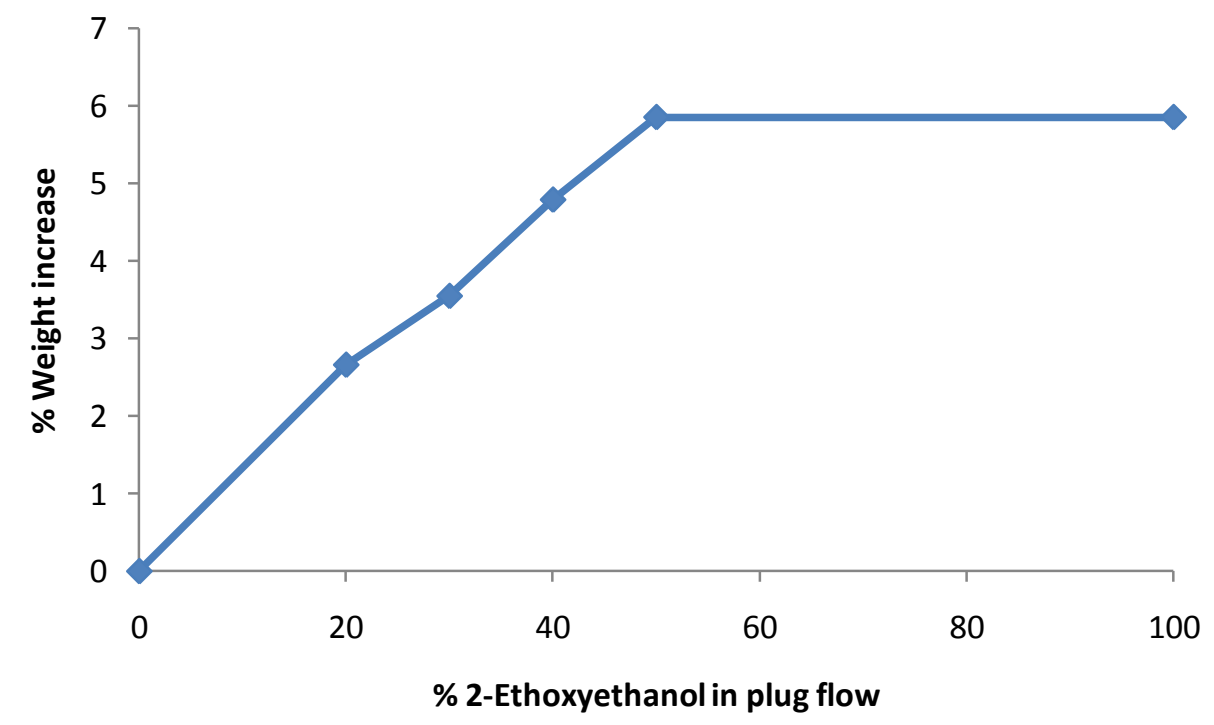

Figure 4.5: The effect of 2-ethoxyethanol upon the weight of dried product.

Thermogravimetric analysis of a sample that had been washed with 2ethoxyethanol also shows a larger overall weight loss than the same material only washed with water (Figure 4.6). Both samples had previously been dried 
at $110{ }^{\circ} \mathrm{C}$ so that there is little difference in the loss of physically bound water up to this temperature. The additional weight loss of the 2-ethoxyethanol washed sample is probably as a result of the higher molecular weight of 2ethoxyethanol compared with that of water, and the 2-ethoxyethanol treated material possessing more groups on the surface due to the larger surface area. Shaw (2000a) determined the weight loss up to $250{ }^{\circ} \mathrm{C}$ from hydrated calcium silicates to be due to loosely bound water. Therefore the significant weight loss observed only for the 2-ethoxyethanol washed material between the temperatures of 250 to $350{ }^{\circ} \mathrm{C}$ (while the water washed material plateaus), is likely to be temperature range the bound 2-ethoxyethanol is volatilised from the surface of the platelets and the cavities between the layers. Above $500{ }^{\circ} \mathrm{C}$, the elimination of water occurs as a result of the condensation of silanol groups to siloxane bonds (Shaw, 2000a). The decomposition of any calcium carbonate impurity to calcium oxide and the release of carbon dioxide will also occur above $600{ }^{\circ} \mathrm{C}$ (Earnest, 1988).

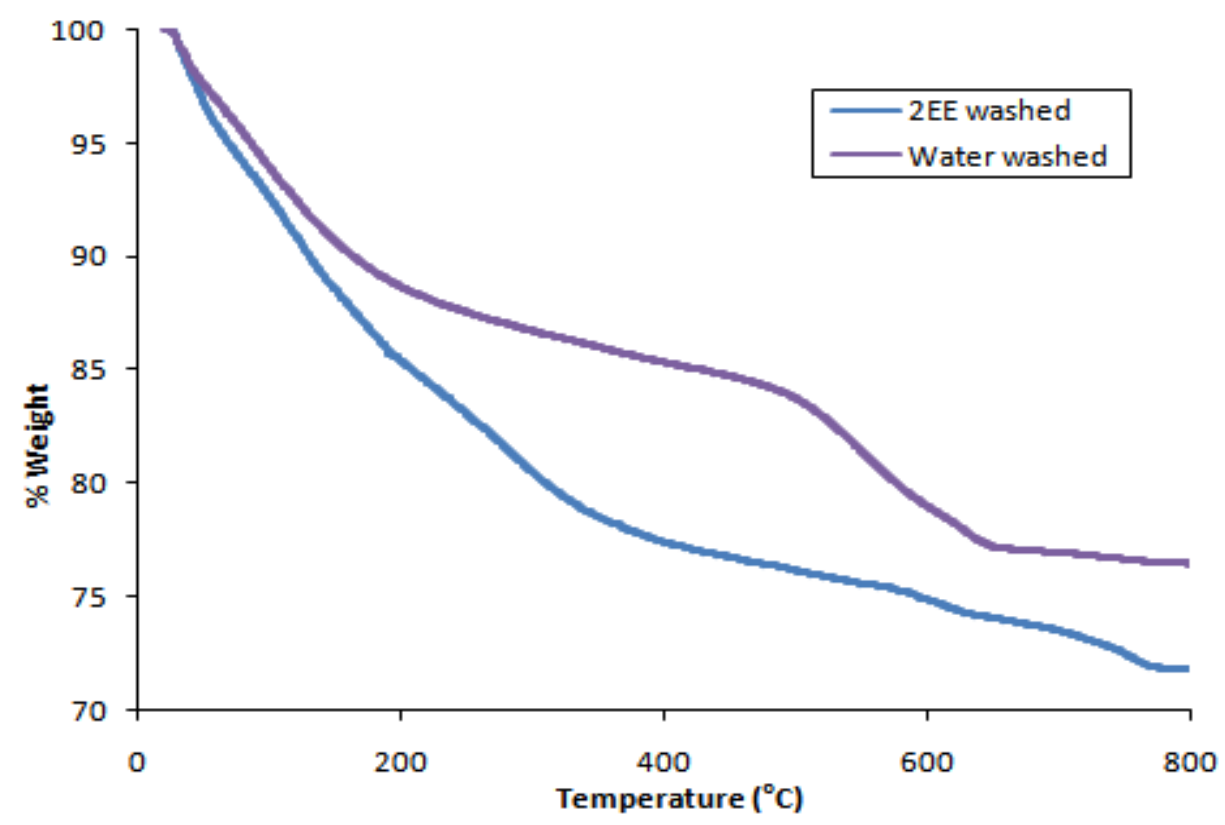

Figure 4.6: Thermogravimetric analysis of a nano-structured calcium silicate that had been washed with 2-ethoxyethanol and the same material washed only with water.

The use of 2-ethoxyethanol to prevent structural collapse has become an important technique for the analysis of nano-structured calcium silicate. This simple, one-step process was essential for the understanding of the ageing process as discussed previously (section 3.2) and has been the common 
technique used for understanding the effect of changing experimental variables upon the structure of resulting materials. While the use of 2-ethoxyethanol has become vital for the analysis of nano-structured calcium silicate, there are limitations on how the resulting material may be used in practical applications. Probably the greatest limitation to using 2-ethoxyethanol to obtain an open structure is that its effect is not permanent, especially due to the hygroscopic nature of the material. When a material that had been treated with 2ethoxyethanol and subsequently dried is submerged in water, the 2ethoxyethanol bound to the structure is exchanged for water so that when the material is dried again the open framework will collapse as a result of surface tension. This collapse of the structure is also observed if the material is left in standard atmospheric conditions due to nano-structured calcium silicate adsorbing moisture from the air and then releasing the water as the humidity fluctuates over time. Under standard conditions the material adsorbs water from the air, which then displaces some of the 2-ethoxyethanol bound to the structure as the water present is in excess therefore driving the equilibrium to favour its co-ordination. When the atmospheric humidity decreases due to natural variations in the environment, the material releases some of the adsorbed water which results in an irreversible partial collapse of the structure that becomes progressively worse over time as the adsorption and loss of water continually cycles. Due to this decrease of the total pore volume over time in commonplace atmospheric conditions, nano-structured calcium silicate treated with 2-ethoxyethanol is more suitable in an applications whereby the material would not be exposed to these conditions for prolonged periods of time. One example of a possible application would be as a carrier material such as containing a phase-change hydrocarbon. This would allow for a liquid to be absorbed shortly after drying while the porosity is at its maximum and the filling of the pores with the hydrocarbon will prevent collapse of the structure when the material is later exposed to conditions that would otherwise lead to collapse as long as the pores filled with this other material.

As a result of the observation that nano-structured calcium silicate absorbs moisture from the air under standard conditions in the laboratory, the effect of 
placing the material in conditions of different humidities was studied. A basic experiment was set-up whereby a nano-structured calcium silicate that had been treated with 2-ethoxyethanol and dried at $110{ }^{\circ} \mathrm{C}$, was placed in an open dish that was suspended above a supersaturated salt solution inside a sealed jar (Figure 4.7).

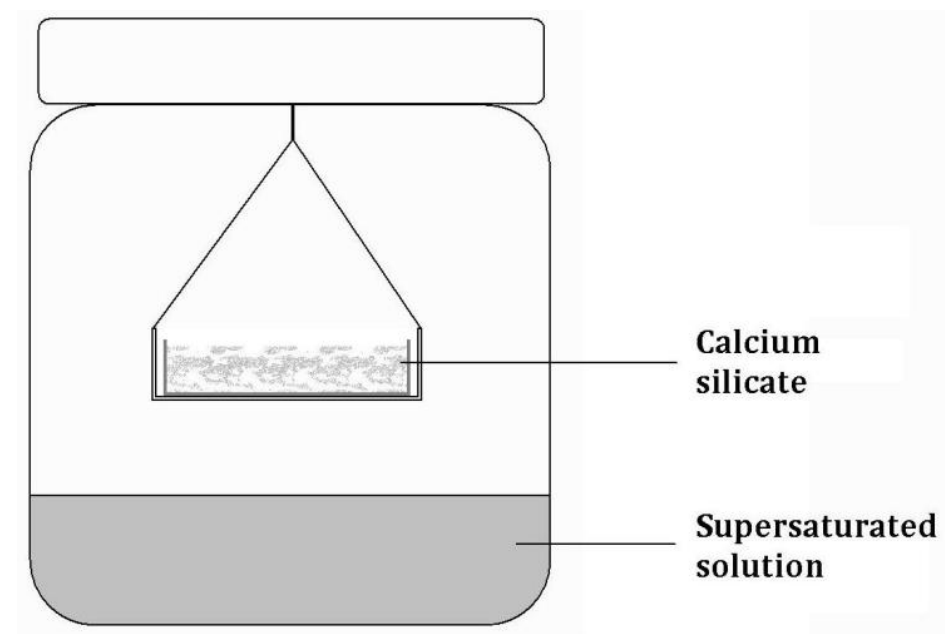

Figure 4.7: A schematic of the experimental set-up for exposing nano-structured calcium silicate to constant relative humidities.

Two supersaturated salt solutions were used to provide different environments of constant relative humidity. A supersaturated solution of sodium chloride was used to give a constant relative humidity of $75 \%$, while a supersaturated calcium chloride solution results in 29\% relative humidity (Lide, 2005). The silicate was placed inside a jar containing a supersaturated solution and periodically removed and weighed until a constant mass was achieved before the sample was then transferred to a jar of different humidity and the process repeated. By cycling the material between high and low humidity conditions, it was observed that maximum \% weight gain (based on the materials weight when dried at $110{ }^{\circ} \mathrm{C}$ ) achieved in high humidity conditions decreased with each cycle until stabilising at ca. $13.2 \%$ from an initial weight gain of $17.6 \%$ (Figure 4.8). This is likely to be due to the collapse of the structure's open framework as the 2-ethoxyethanol is displaced, therefore decreasing the surface area and reducing the number of sites to which water molecules may bind. Since the amount of water adsorbed and desorbed eventually becomes constant in the different relative humidity conditions, there is potential that the material could be used for passive humidity control whereby the material 
would act as a buffer in reducing the variations of humidity observed in a given environment.

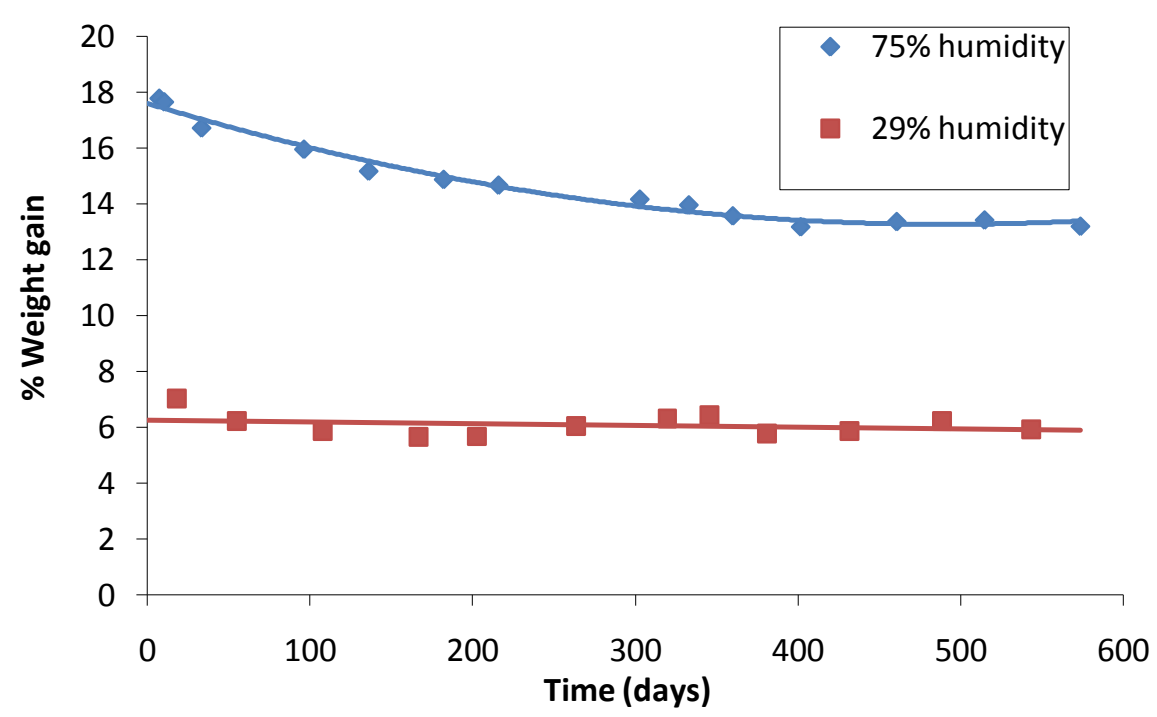

Figure 4.8: Weight gain of nano-structured calcium silicate due to the adsorption of water under $75 \%$ and $29 \%$ constant relative humidity conditions relative to the materials weight when dried at $110^{\circ} \mathrm{C}$.

The tendency of the material treated with 2-ethoxyethanol to decrease in pore volume when reintroduced to an aqueous environment prevents its use as a paper filler or coating which were seen as among the main applications of the material. This is because of the method used to apply the material to the sheet would need to be compatible with the current methods of sheet formation that use water as the solvent. The result of going through this process would be a material that has a greatly diminished pore volume which is the property that exceeds the other materials currently used as coating and fillers and therefore reduce its attractiveness as an alternative for this application. Another drawback of using the 2-ethoxyethanol treated nano-structured calcium silicate for this application is the detrimental effect this solvent has upon the optical properties of the material. As the proportion of 2-ethoxyethanol in the plug flow in increased, both the whiteness $\left(\mathrm{L}^{*}\right)$ and the TAPPI brightness (reflectance at $457 \mathrm{~nm}$ ) decrease (Figure 4.9) and the $\mathrm{b}^{*}$ value becomes more positive as the material develops an increasingly yellow hue that can be seen optically (Figure 4.10). 


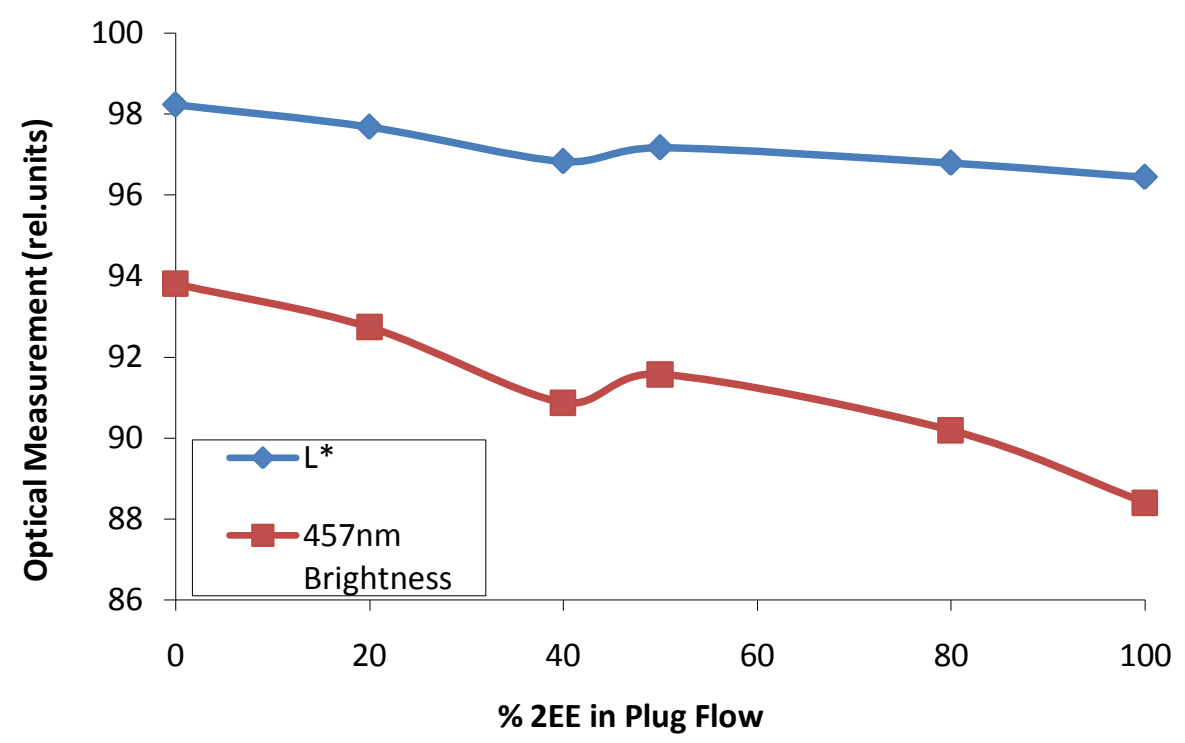

Figure 4.9: The effect of treating nano-structured calcium silicate with 2-ethoxyethanol upon the whiteness and brightness of the material (relative units).

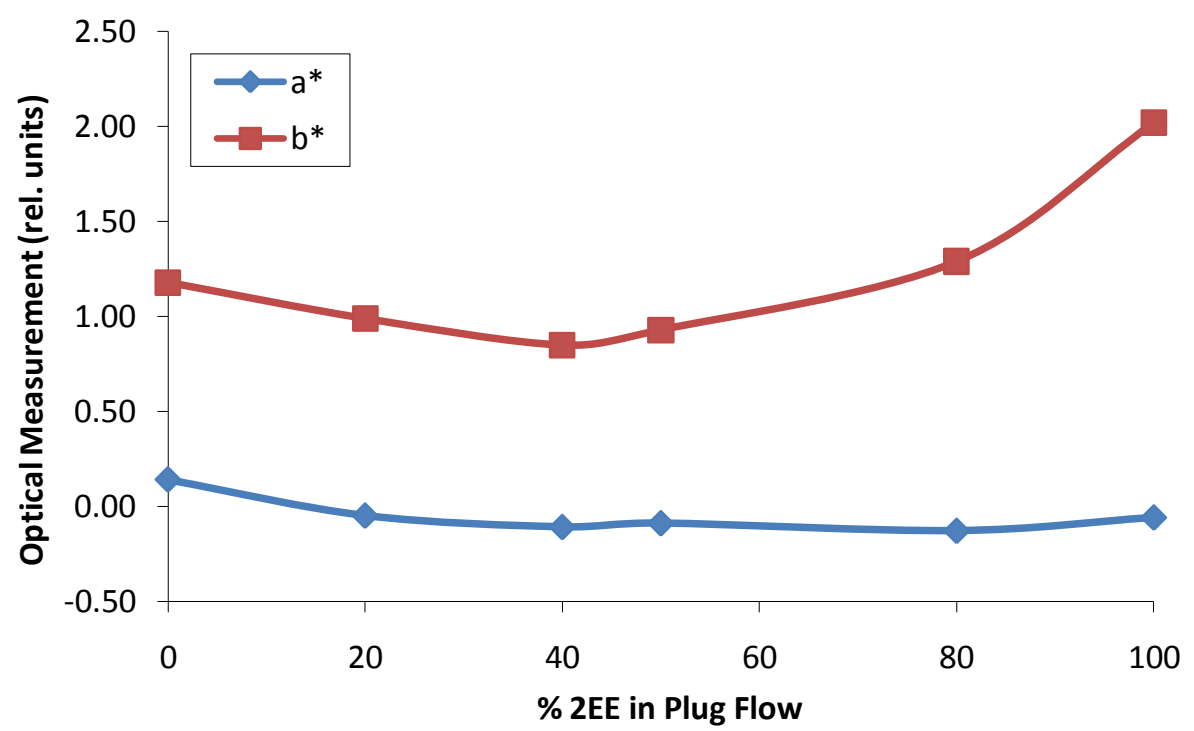

Figure 4.10: The effect of treating nano-structured calcium silicate with 2-ethoxyethanol upon the colour of the material (relative units).

\subsection{Reinforcement of the structure}

The polymerisation of silica upon pre-existing silica gel or precipitated silica network structures to strengthen the structure has been known for a long period of time (Alexander et al., 1956). The source of monomeric silica for the polymerisation may come from either the addition of a soluble silicate or by the 
dissolution of silica from certain parts of the structure (by heating the gel for example) that is subsequently deposited at other parts of the structure. The reinforcement technique utilises the variation of silica solubility with the radius of curvature of silica particles surface (Figure 4.11).

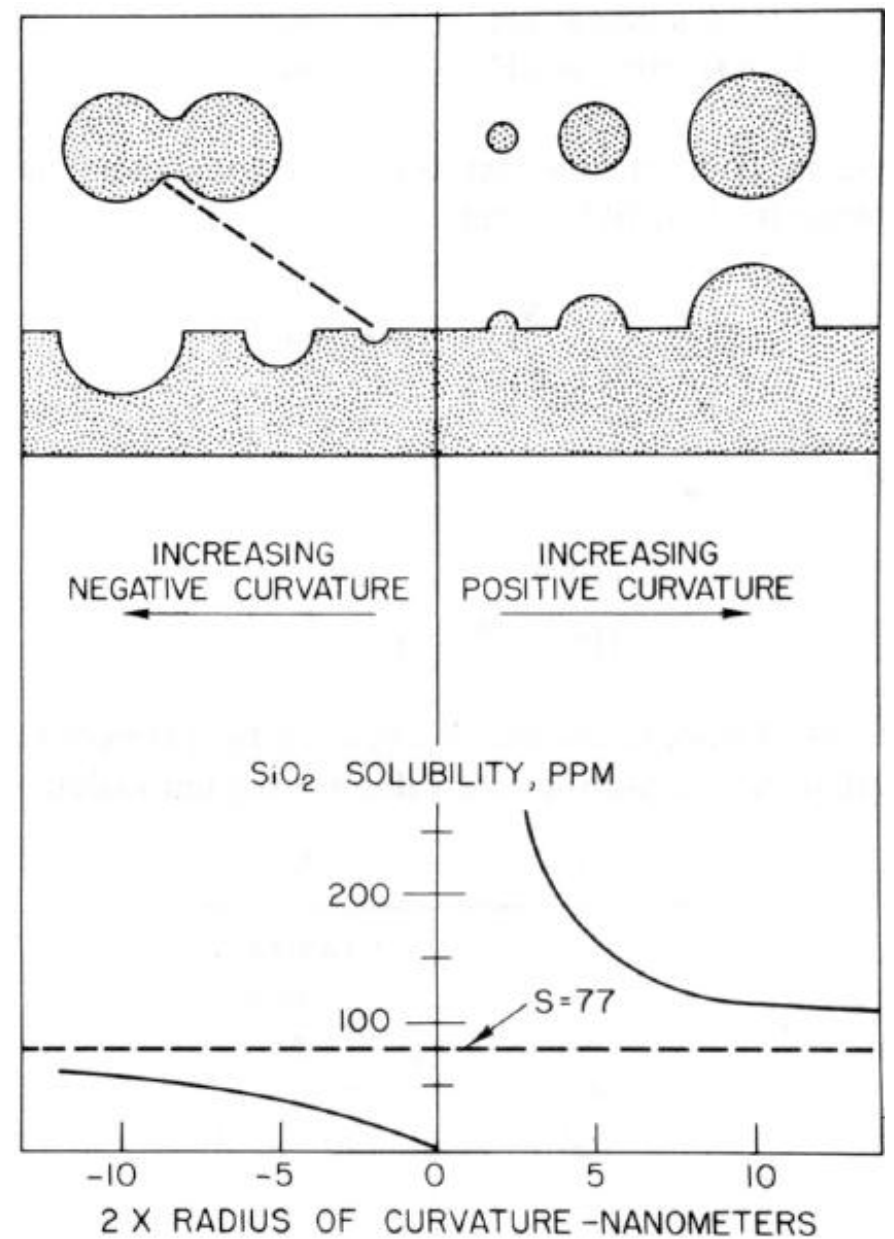

Figure 4.11: Variation in solubility of silica with radius of curvature of surface (Iler, 1979). $S$ is the solubility of a flat surface or a particle of infinite radius.

As a result of the equilibrium solubility being at its highest for small particles (as these particles have a small positive radius of curvature), it is these particles that in suitable conditions dissolve and provide a source of silica. More importantly for the reinforcement technique is that the solubility is at its lowest on surfaces with a narrow negative curvature, therefore any monomeric silica in solution from either a soluble silicate or from dissolved particles will preferentially deposit at these regions. On network silicas these regions of small negative curvature occur where two sol particles have fused together. Any deposition in these regions causes the structure to become more rigid and 
increase in mechanical strength as it is at these points of the structure that failure occurs when forces are applied. It is the breaking of bonds between the sol particles as a result of forces during liquid removal upon drying that leads to the collapse of the structure and a reduction of the pore volume. This can be seen with the force that causes capillary rise, which is analogous to the compressive forces subjected to structures during liquid removal, where the height of the rise is inversely proportional to the radius of the cavity. This behaviour is described by the equation below where $h$ is the height of the column, $\rho$ is the liquid density, $\gamma$ is the surface tension, $r$ is the radius of cavity, and $g$ is the acceleration due to gravity (Bikerman, 1948).

$$
h=\frac{2 \gamma}{r \rho g}
$$

Due to the increase in force exerted with decreasing radius of curvature, the deposition of silica into these regions occupies the parts of the structure where the strongest forces would otherwise exist. The result of reinforcement is that a structure is able to withstand greater capillary and mechanical forces and therefore maintain its pore volume under a wider range of conditions.

While the reinforcement of network structures has been extensively used for the production of silica gels and precipitated silicas, this methodology is not known to have been used for the strengthening of silicate structures. Once it was known that a large proportion of the pore volume of nano-structured calcium silicate was being lost due to collapse of the structure upon liquid removal, the possible use of reinforcement was explored. Like any structure, the compressive force on the material increases with smaller diameter capillaries. As the platelets of nano-structured calcium silicate are randomly arranged, the compressive forces present will differ throughout the structure with the greatest forces likely to occur where plates intersect at an acute angle. If the addition of monomeric silica led to polymerisation in the regions of negative curvature like that observed for network silicas, then similar strengthening of the structure would be likely to occur with the possibility of preventing structural collapse upon liquid removal (Figure 4.12). 

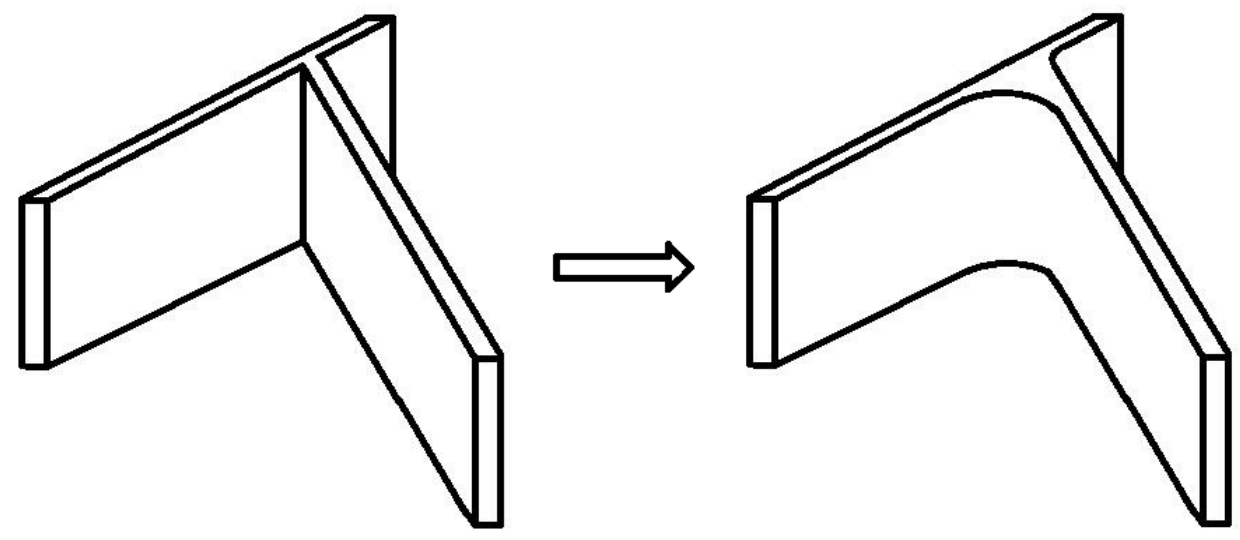

Figure 4.12: A representation of two intersecting nano-structured calcium silicate plates before and after reinforcement.

An initial study was carried out by adding additional amounts of sodium silicate to sub-samples from a nano-structured calcium silicate slurry that was produced using the batch process using $0.58 \mathrm{~mol} / \mathrm{L}$ (34800 mg/L) monomeric silica, $0.62 \mathrm{~mol} / \mathrm{L}$ calcium ions (25000 mg/L), and $0.34 \mathrm{~mol} / \mathrm{L}$ hydrochloric acid and aged overnight. Each sample was only washed with water before drying at $110{ }^{\circ} \mathrm{C}$ and the oil absorption capacity measured to determine if the reinforcement had prevented collapse of the structure. The addition of increasingly higher concentrations of monomeric silica resulted in materials with increasingly larger oil absorption capacities (Figure 4.13).

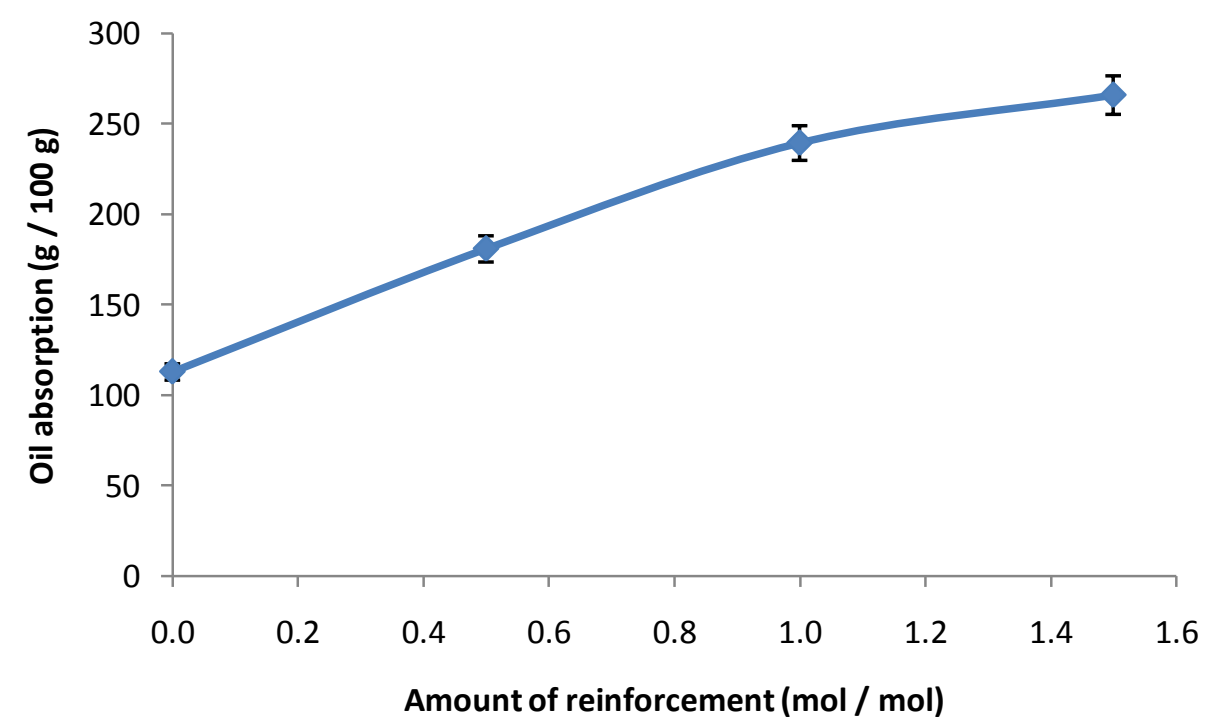

Figure 4.13: Effect of reinforcement upon the oil absorption capacity. 
The proportion of monomeric silica used for reinforcement is given as moles of additional monomeric silica added for reinforcement per moles of monomeric silica used for the formation of the nano-structured calcium silicate (based on the assumption that approximately all of the silica used initially is included in the material formed). Due to the effect of the additional monomeric silica upon the oil absorption capacity, it can be assumed that the reinforcement of nanostructured calcium silicate occurs in a similar way to that of network silicas. The oil absorption capacity was found to increase linearly before starting to plateau due to the monomeric silica no longer polymerising onto the surface as indicated by the increase of monomeric silica remaining in solution as the amount of sodium silicate added increased (Figure 4.14).

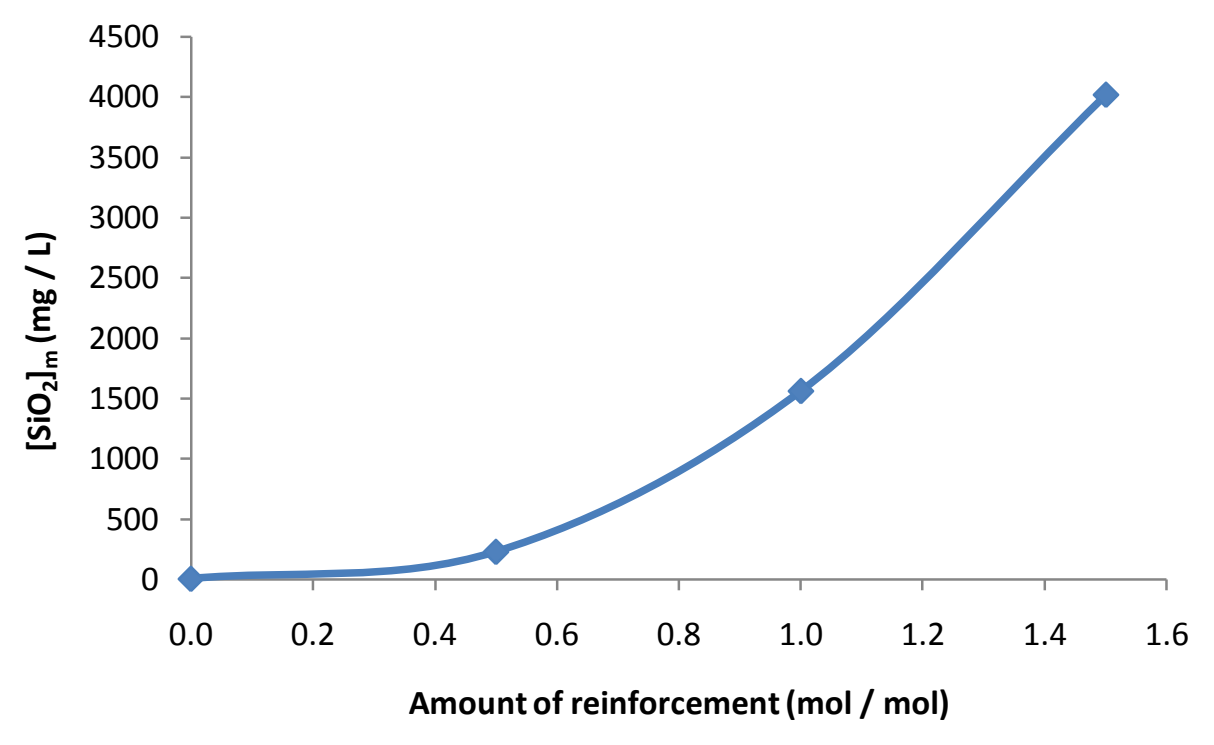

Figure 4.14: The concentration of monomeric silica remaining in solution following reinforcement.

The oil absorption capacity at levels of reinforcement above $1.4(\mathrm{~mol} / \mathrm{mol})$ remained constant, indicating that the maximum level of reinforcement had been reached. The cessation of further silica addition onto the surface when higher concentrations of monomeric silica are added can also be seen through measuring the composition of the materials using X-ray fluorescence spectroscopy (Figure 4.15). The $\mathrm{Si} / \mathrm{Ca}$ molar ratio increases for low reinforcement concentrations as is expected for the deposition of silica onto the structure, but the addition of further monomeric silica does not alter the 
composition of the material with the curve flattening out as the additional silica is not polymerised onto the structure.

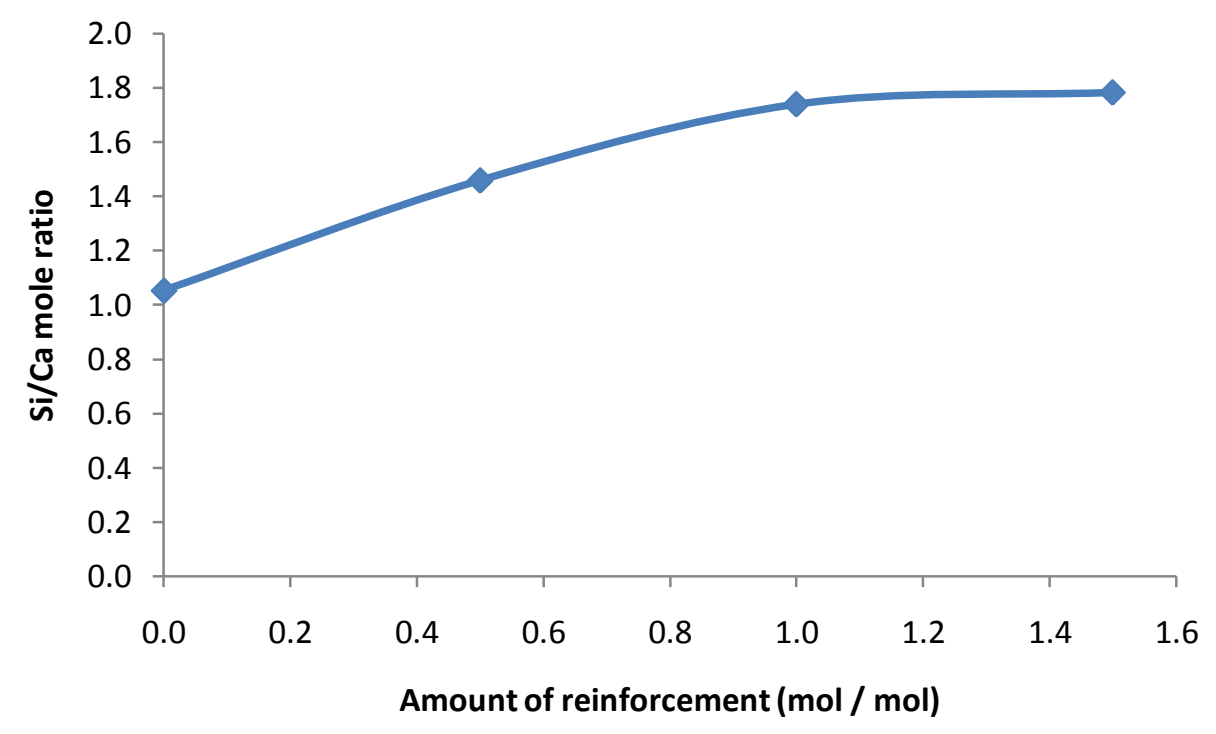

Figure 4.15: The effect of reinforcement upon the composition of the resulting nano-structured calcium silicate.

It is assumed that effectively all of the polymerisation occurs upon the preexisting nano-structured calcium silicate structure as the $\mathrm{pH}$ of the reaction is above that at which the formation of a network structure would occur. The existing silicate would also likely provide good sites for nucleation, with the areas of negative curvature providing regions of lower solubility. The X-ray powder diffraction pattern of the material that has been reinforced shows no significant difference to that of the base untreated material (Figure 4.16). This indicates that the structure of the base material is unaffected by the process, confirming that only the surface is modified. Both samples contained an impurity of calcium carbonate (PDF: 05-0586) which obscures the main calcium silicate hydrate peak. The absence of a significant amount of independently polymerised silica particles in the reinforced sample can be concluded from the lack of a broad peak centred between 15 to $30^{\circ}$ that is typically observed for amorphous silicas. 


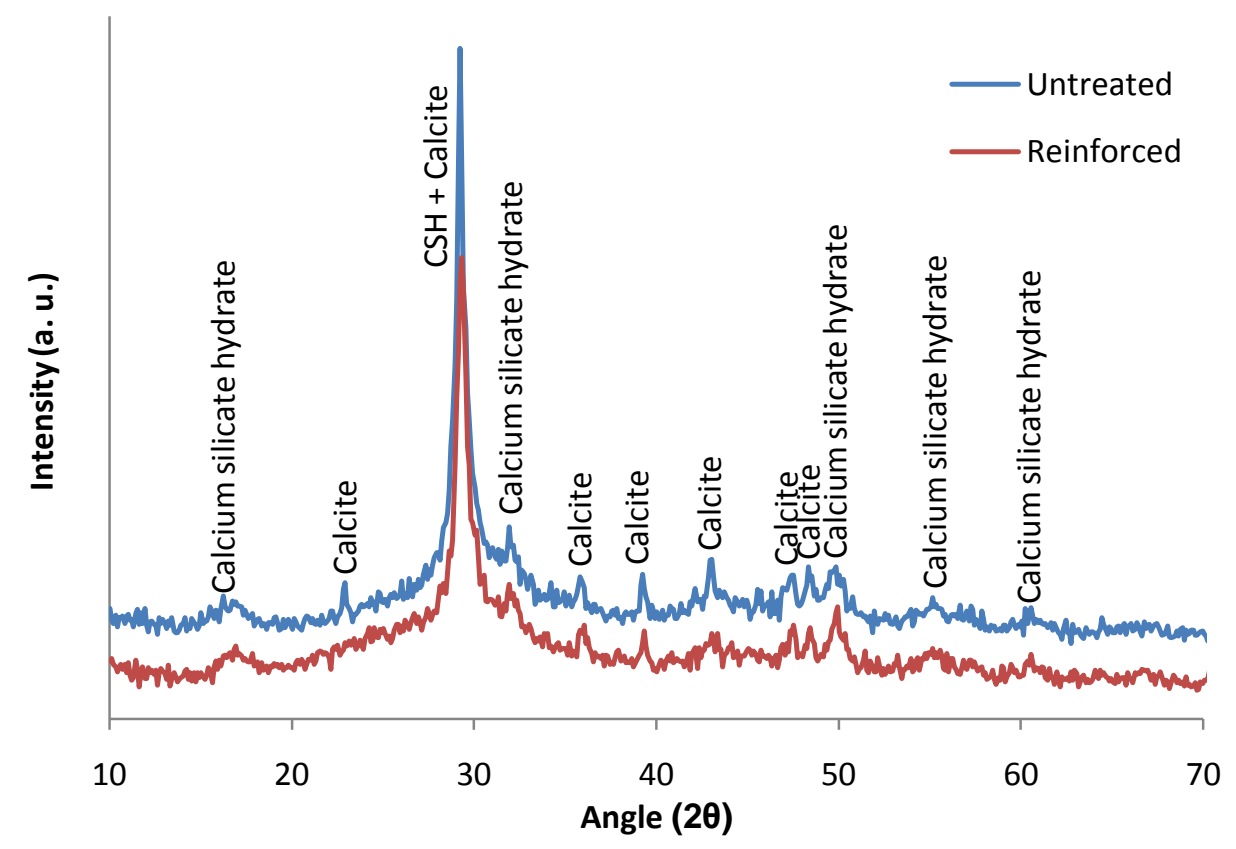

Figure 4.16: X-ray powder diffractograms of nano-structured calcium silicate before and after reinforcement.

The rate of the reinforcement reaction was studied by monitoring both the concentration of unreacted monomeric silica remaining in solution and the effect of the reinforcement upon the resulting physical properties over time. The concentration of monomeric silica in solution was found to be approximately constant from the time the first sample was taken after 5 minutes until the final sample was taken after 4 days (Figure 4.17).

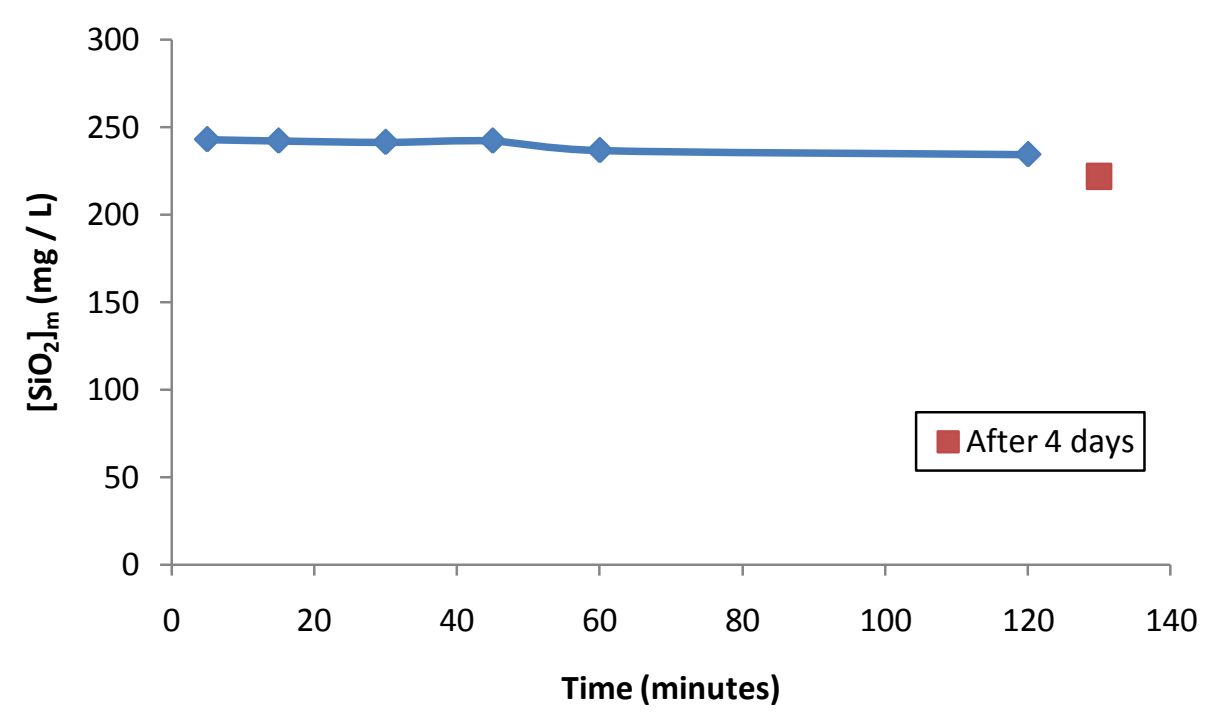

Figure 4.17: Concentration of unreacted monomeric silica remaining in solution during the reinforcement reaction. 
This concentration is probably the solubility of amorphous silica at the $\mathrm{pH}$ and temperature of the reaction conditions. The oil absorption capacity and surface area of samples taken over time were also found to remain constant from the time the first sample was taken after 5 minutes (Figure 4.18). Both of these results indicate that the reinforcement reaction is very rapid and complete within 5 minutes. This could be explained by the large amount of surface that is available for the monomeric silica to polymerise onto.

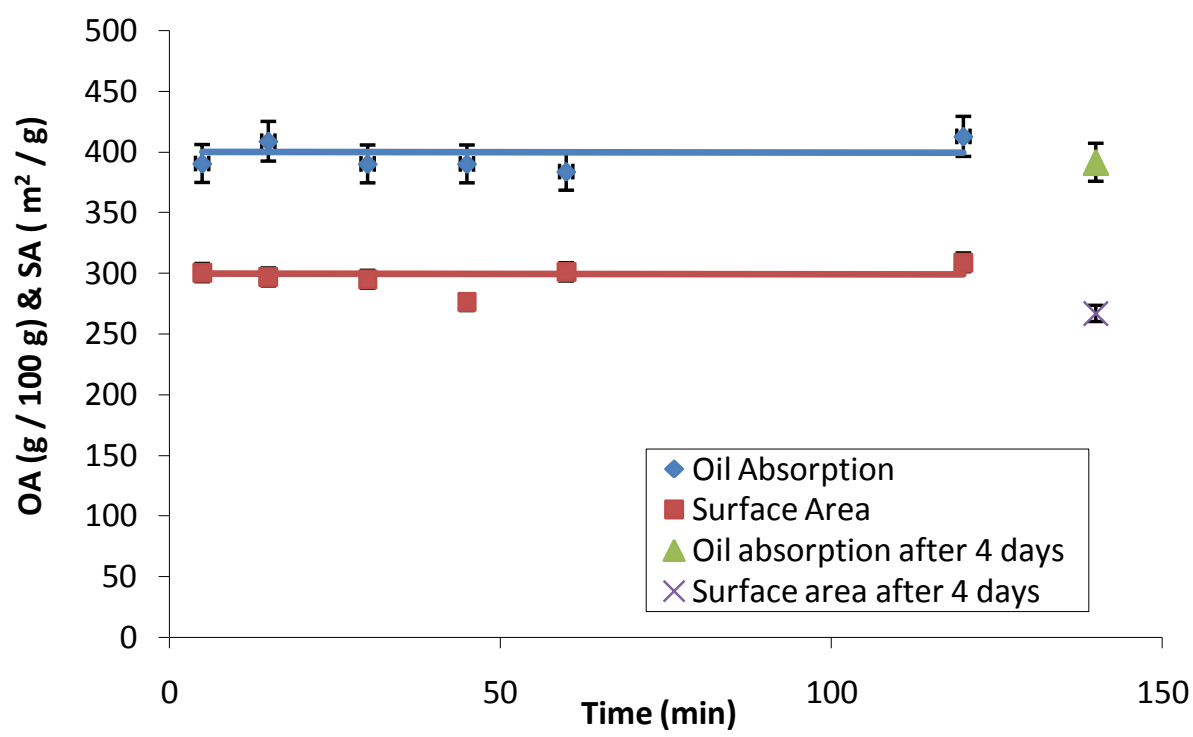

Figure 4.18: Development of the oil absorption capacity and surface area during the reinforcement reaction.

In the previous reinforcement experiments the difference in solubility of different parts of the structure determined where the polymerisation occurred and the amount of polymerisation that can take place. As can be seen in Figure 4.13, while the maximum oil absorption capacity had been achieved using a 1 $\mathrm{mol} / \mathrm{mol}$ proportion of reinforcement, Figure 4.14 shows excess monomeric silica remains in solution. In order to polymerise more of the monomeric silica available in solution, a study into the effect of decreasing the $\mathrm{pH}$ of the slurry prior to reinforcement was undertaken as the solubility would be reduced at a lower $\mathrm{pH}$. The relatively lower solubility of areas with negative curvature upon the structure should cause the majority of polymerisation to still occur on the nano-structured calcium silicate rather than form independent silica particles. However, the formation of silica particles is more likely to occur at this lower 
$\mathrm{pH}$ than in conditions where the $\mathrm{pH}$ had not been adjusted, especially when high concentrations of monomeric silica are added.

A base nano-structured calcium silicate was prepared using the continuous process with $0.32 \mathrm{~mol} / \mathrm{L}$ monomeric silica, $0.31 \mathrm{~mol} / \mathrm{L}$ calcium ions, and 0.17 mol/L hydrochloric acid and allowed to fully age. To $50 \mathrm{~mL}$ samples of this slurry, varying amounts of sodium silicate were added followed by the immediate addition of $4 \mathrm{~mL}$ of $2 \mathrm{~mol} / \mathrm{L}$ hydrochloric acid (detailed conditions are given in Table 4.2).

Table 4.2: Conditions for reinforcement at ca. $\mathrm{pH} 10$

\begin{tabular}{cccc}
\hline $\begin{array}{c}\text { Weight of } \\
\text { sodium silicate } \\
\text { added (g) }\end{array}$ & $\begin{array}{c}\text { Amount of silica } \\
\text { added (mol) }\end{array}$ & $\begin{array}{c}\text { Proportion of } \\
\text { silica added } \\
\text { (mol/mol) }\end{array}$ & $\begin{array}{c}\text { Final pH of } \\
\text { slurry }\end{array}$ \\
\hline 0.0 & 0.000 & 0.00 & 9.5 \\
0.5 & 0.002 & 0.14 & 9.5 \\
1.0 & 0.004 & 0.27 & 9.6 \\
1.5 & 0.007 & 0.41 & 9.6 \\
2.0 & 0.009 & 0.55 & 9.5 \\
2.5 & 0.011 & 0.68 & 9.6 \\
3.0 & 0.013 & 0.82 & 10.0 \\
3.5 & 0.015 & 0.96 & 10.2 \\
4.0 & 0.018 & 1.10 & 10.3 \\
\hline
\end{tabular}

This amount of acid was sufficient to lower the pH of the slurry to 9.5 for the lowest amount of sodium silicate and to 10.3 for the greatest additional amount of sodium silicate added. Due to the buffering effect of nano-structured calcium silicate the $\mathrm{pH}$ can take hours to stabilise, therefore a specific amount of acid was added to obtain an approximate $\mathrm{pH}$ of 10 rather than actively trying to adjust the $\mathrm{pH}$ to the exact value. A pH of approximately 10 was chosen as the desired $\mathrm{pH}$ because polymerisation occurs most readily when the $\mathrm{pH}$ equals the $\mathrm{pK}_{\mathrm{a}}$ of monosilicic acid ( $\mathrm{pH}$ 9.9). As discussed previously the solubility of amorphous silica rapidly decreases at room temperature at $\mathrm{pH} 10$ (Figure 1.6) and conditions are therefore ideal to polymerise silica. The general shape of the curve for the change in oil absorption capacity of these reinforced samples with increasing amounts of silica added resembles that achieved without any 
adjustment to the $\mathrm{pH}$ with a linear increase initially followed by a plateau (Figure 4.19).

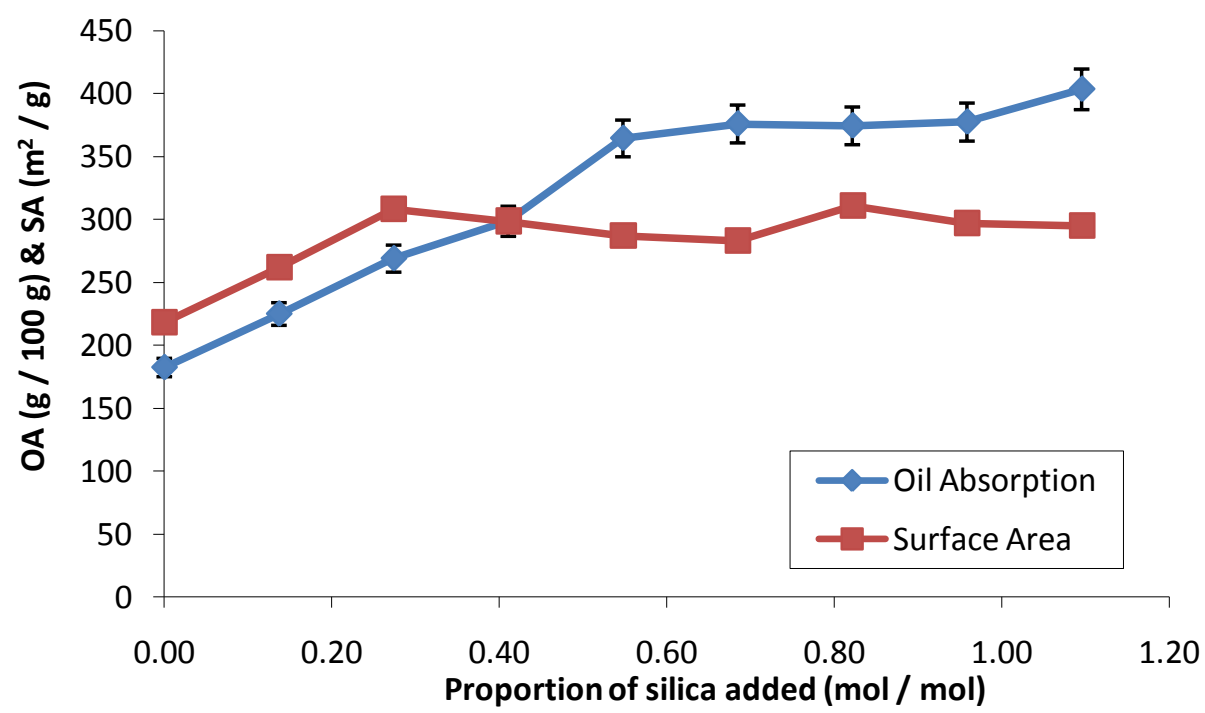

Figure 4.19: Effect of reinforcement upon physical properties at ca. pH 10.

However, the plateau of the reinforcement at ca. $\mathrm{pH} 10$ is higher with an oil absorption capacity of $375 \mathrm{~g} / 100 \mathrm{~g}$ (rather than $250 \mathrm{~g} / 100 \mathrm{~g}$ ) and this higher plateau is reached with a lower amount of monomeric silica $0.6 \mathrm{~mol} / \mathrm{mol}$ (rather than $1.0 \mathrm{~mol} / \mathrm{mol}$ ). The surface area of the material was also found to follow a trend similar to that observed for the oil absorption capacity in response to the increase degree of reinforcement. As intended by decreasing the $\mathrm{pH}$ of the reinforcement, the concentration of silica remaining in the solution following the reaction is greatly reduced (Figure 4.20).

Like the reinforcement reactions at the higher $\mathrm{pH}$, the increase in remaining monomeric silica occurs at the proportion of silica added where there is no further improvement in oil absorption capacity $(0.6 \mathrm{~mol} / \mathrm{mol})$. This increase in monomeric silica concentration is also likely to be a result of the $\mathrm{pH}$ of the slurry increasing as the concentration of sodium silicate added increases, thereby increasing the solubility of amorphous silica (Figure 1.6). As a result of adding more silica to the surface during the reinforcement process, the weight of product increased (Figure 4.21). The increase in the weight of product 
formed exceeded that of the additional amount of monomeric silica added in all cases except when the highest amount of silica was added (Table 4.3).

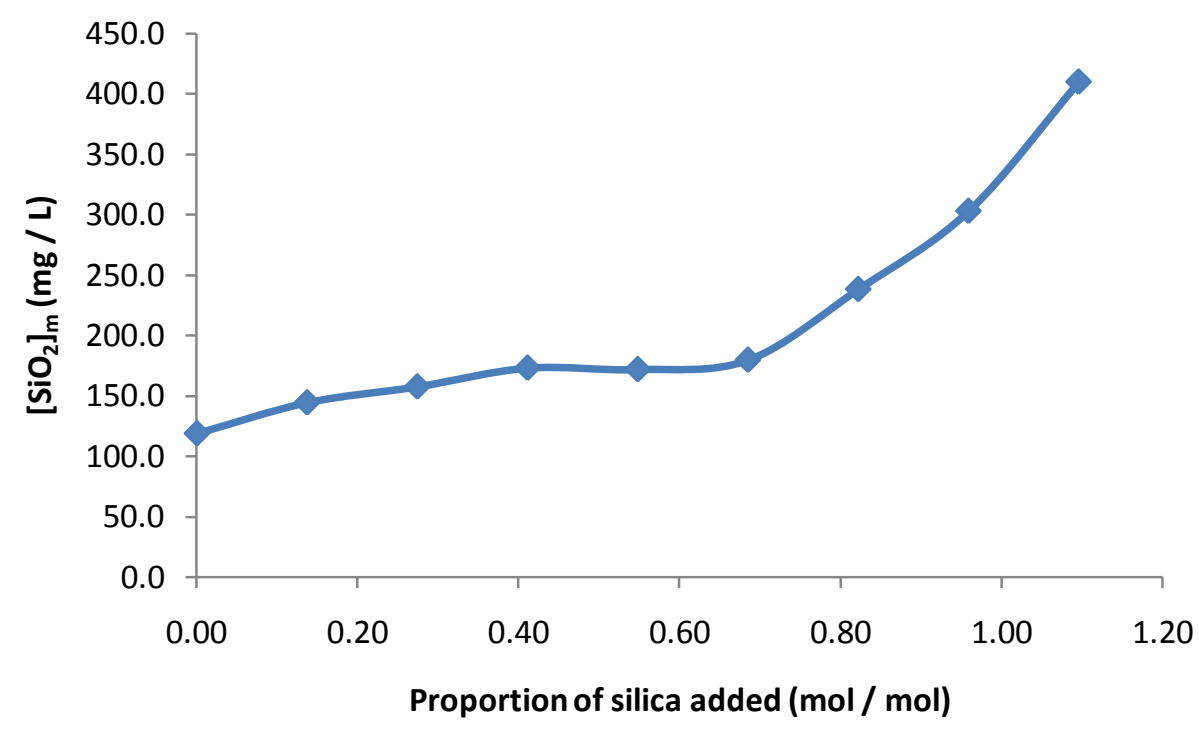

Figure 4.20: Monomeric silica remaining in solution following reinforcement at ca. pH 10.

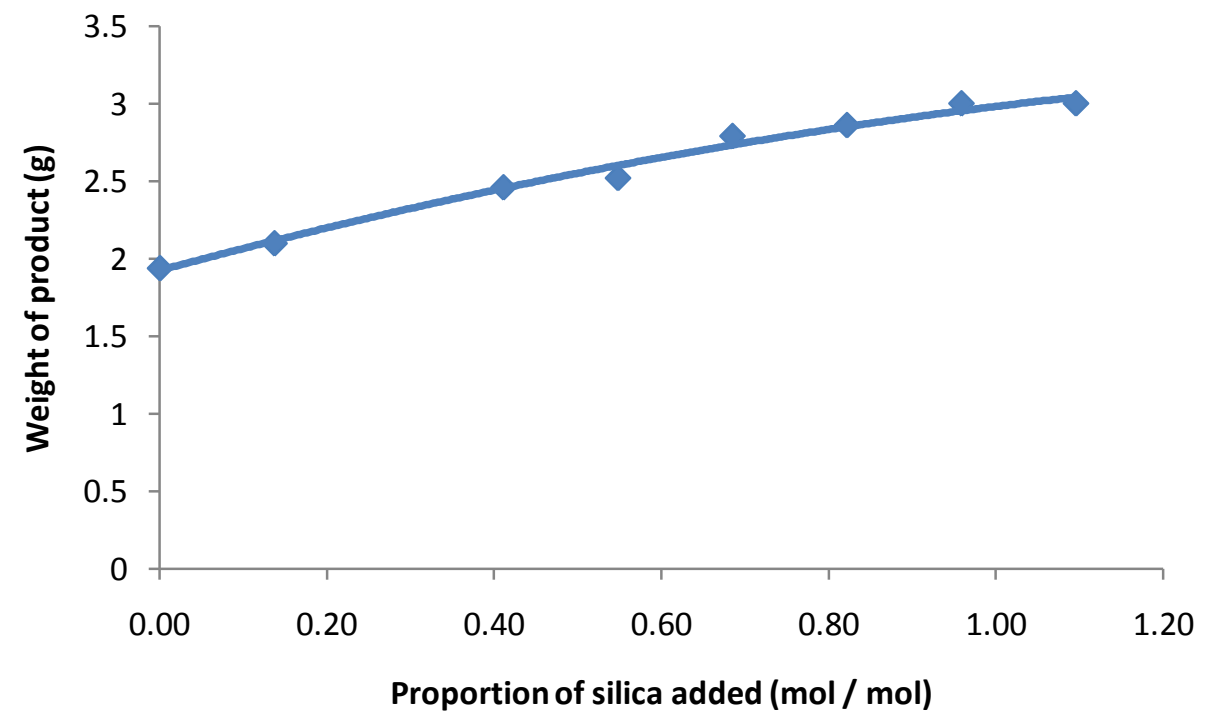

Figure 4.21: Increase in product weight due to reinforcement.

This additional increase is likely to be a result of the increase in surface area as more reinforcement occurs that therefore increases the amount of that water that can bind to the material. The mass of the sample reacted with highest concentration of monomeric silica did not increase by at least the weight of additional monomeric silica added due to not all of the silica being deposited (as evidenced by the increased monomeric silica in solution in Figure 4.20). 
Table 4.3: Comparison of the increased weight of product with the weight of monomeric silica added.

\begin{tabular}{ccc}
\hline $\begin{array}{c}\text { Proportion of silica } \\
\text { added (mol/ mol) }\end{array}$ & $\begin{array}{c}\text { Weight of silica } \\
\text { added }(\mathrm{g})\end{array}$ & $\begin{array}{c}\text { Increase in weight of } \\
\text { product }(\mathrm{g})\end{array}$ \\
\hline 0.00 & 0.00 & 0.00 \\
0.14 & 0.14 & 0.16 \\
0.41 & 0.43 & 0.52 \\
0.55 & 0.57 & 0.58 \\
0.68 & 0.71 & 0.85 \\
0.82 & 0.86 & 0.92 \\
0.96 & 1.00 & 1.06 \\
1.10 & 1.14 & 1.06 \\
\hline
\end{tabular}

Following the improvement of the oil absorption capacity at ca. $\mathrm{pH} \mathrm{10,} \mathrm{a} \mathrm{more}$ detailed study was performed to optimise the conditions. This study focussed on slightly changing in the proportion of monomeric silica added around the point where the oil absorption capacity started to plateau and the amount of unreacted monomeric silica in solution started to increase. The study also involved small adjustments to the $\mathrm{pH}$ that the reinforcement occurred at by varying the volume of hydrochloric acid added following the addition of sodium silicate. A base nano-structured calcium silicate was prepared using the continuous process with $0.43 \mathrm{~mol} / \mathrm{L}$ monomeric silica, $0.47 \mathrm{~mol} / \mathrm{L}$ calcium ions, and $0.25 \mathrm{~mol} / \mathrm{L}$ hydrochloric acid and allowed to fully age. To $50 \mathrm{~mL}$ samples of this slurry, varying amounts of sodium silicate $(2.5,3.0,3.5$, and 4.0 g) were added followed by the immediate addition of varying volumes of $2 \mathrm{~mol} / \mathrm{L}$ hydrochloric acid $(3.0,4.0,5.0$, and $6.0 \mathrm{~mL})$ to give slurries of varying $\mathrm{pHs}$ as detailed in Table 4.4

Table 4.4: The pH of nano-structured calcium silicate slurries after reinforcement using different combinations of monomeric silica and hydrochloric acid

Volume of $2 \mathrm{M} \mathrm{HCl}$ added (mL)

\begin{tabular}{cc|cccc} 
& & 3 & 4 & 5 & 6 \\
\cline { 2 - 6 } Proportion of & 0.51 & 10.1 & 9.8 & 9.5 & 9.4 \\
monomeric & 0.61 & 10.1 & 9.8 & 9.6 & 9.4 \\
silica added & 0.71 & 10.3 & 10.0 & 9.7 & 9.5 \\
(mol/mol) & 0.82 & 10.5 & 10.1 & 9.8 & 9.6
\end{tabular}


Over this narrower range of conditions, the materials prepared did not differ significantly in terms of their physical properties with both the oil absorption capacities and surface areas similar for all combinations of the reaction $\mathrm{pH}$ and amount of monomeric silica added (Figure 4.22 and Figure 4.23).

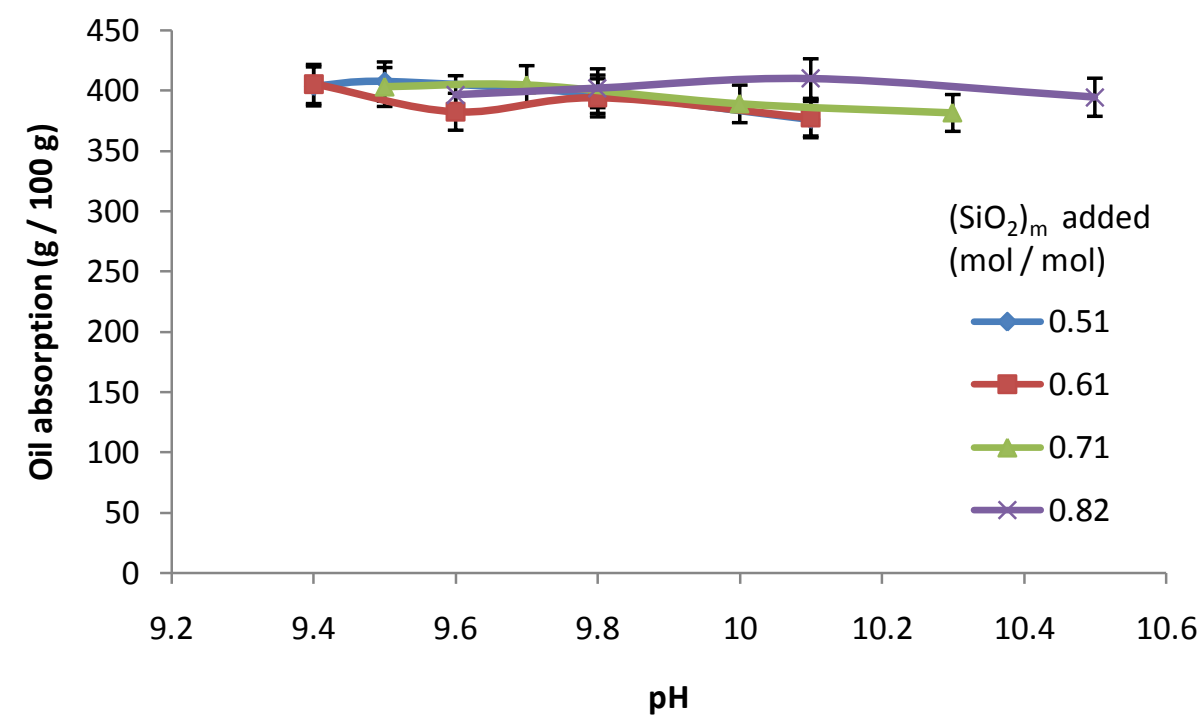

Figure 4.22: The effect of reaction $\mathrm{pH}$ upon the oil absorption capacity of nano-structured calcium silicate reinforced with different amounts of monomeric silica.

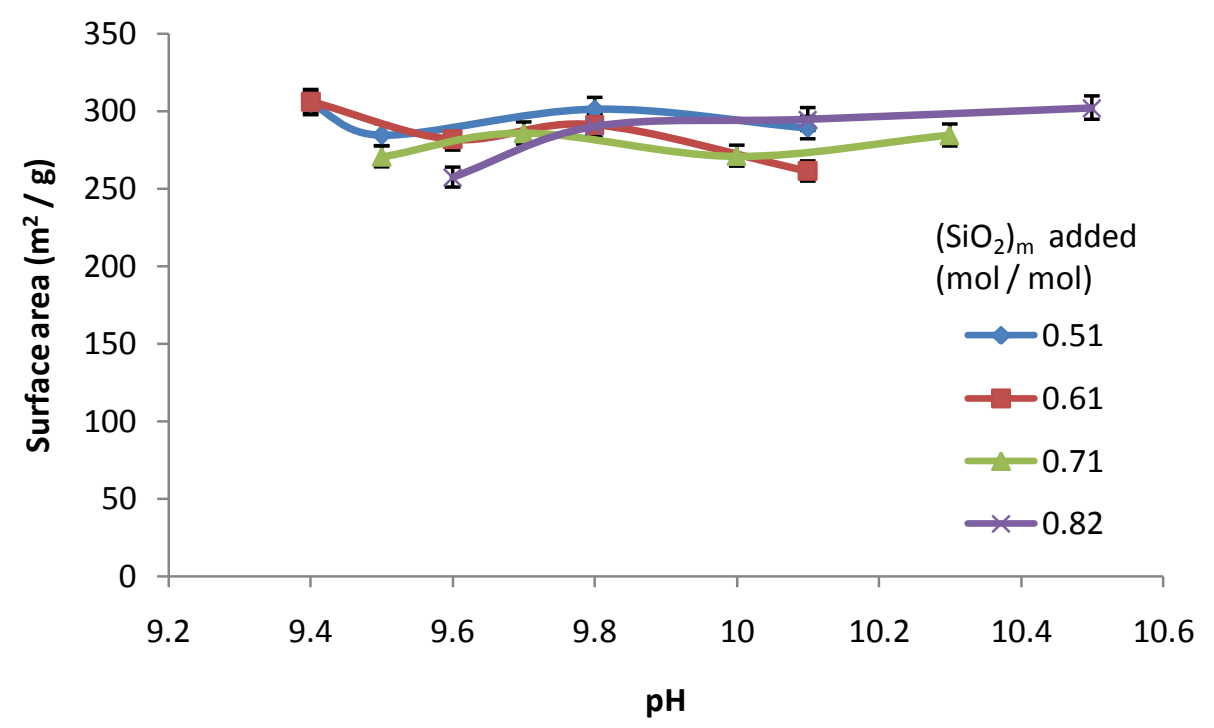

Figure 4.23: The effect of reaction $\mathrm{pH}$ upon the surface area of nano-structured calcium silicate reinforced with different amounts of monomeric silica.

These results indicate that the properties of reinforced nano-structured calcium silicate are not overly sensitive to either the $\mathrm{pH}$ of the reaction or the amount of monomeric silica added. This is advantageous for producing the 
material at larger scales where control of reaction conditions may not be as precise as performing the reaction on a bench-top scale.

Analysis of the concentration of monomeric silica and calcium ions remaining in solution after the reaction confirmed the expected trends. The highest concentration of monomeric silica was measured for the sample reinforced with the highest proportion of monomeric silica added and each reaction performed with less monomeric silica progressively resulted in less unreacted monomeric silica (Figure 4.24). The concentration of monomeric silica remaining in solution following the reaction was also found to decrease as the amount of acid added increased as a result of the solubility of silica decreasing with $\mathrm{pH}$.

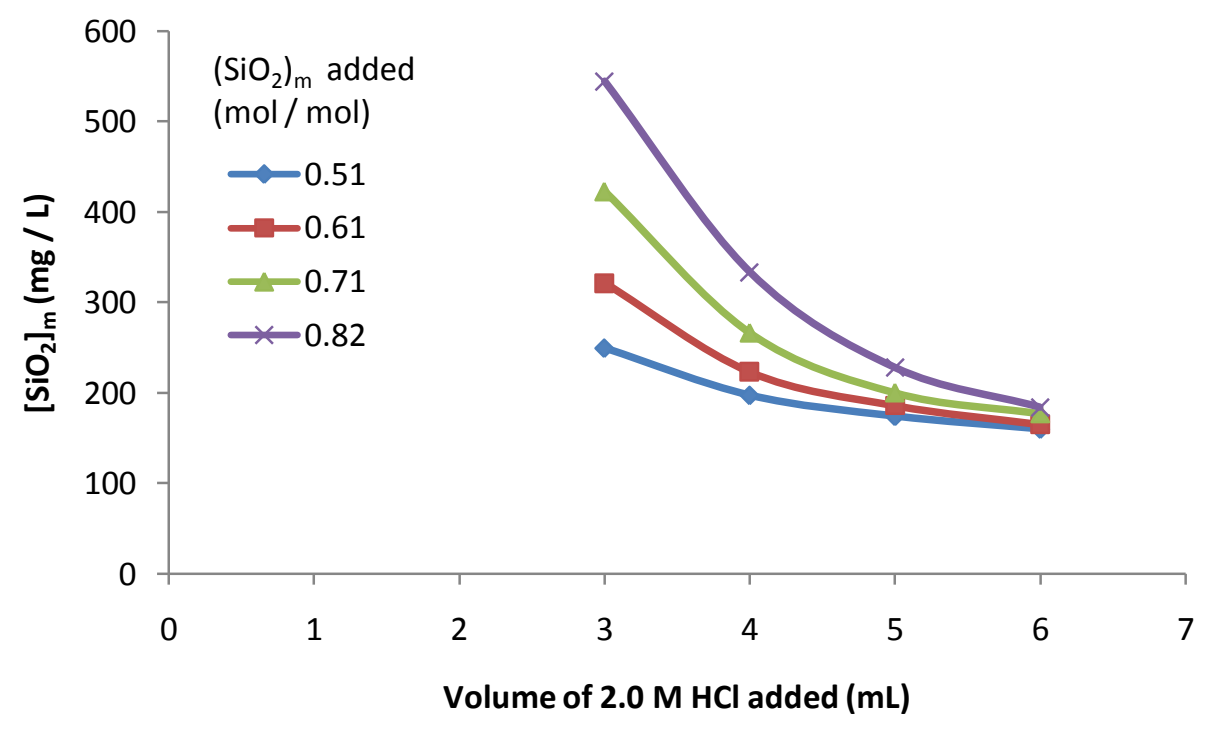

Figure 4.24: The concentration of monomeric silica remaining in solution after reinforcement with varying amounts of monomeric silica and hydrochloric acid.

The calcium ion concentration was found to follow the opposite trends to that of monomeric silica. The concentration of calcium present after the reaction decreased as the proportion of monomeric silica added increased probably due to the solubility of the nano-structured calcium silicate with the additional monomeric silica reacting with residual calcium cations to precipitate more calcium silicate (Figure 4.25). The effect of the addition of more acid to the slurry had the effect of increasing the calcium concentration remaining in 
solution which is likely to be a result of the exchange of calcium in the structure for the additional protons in the solution as detailed further in Section 4.3.

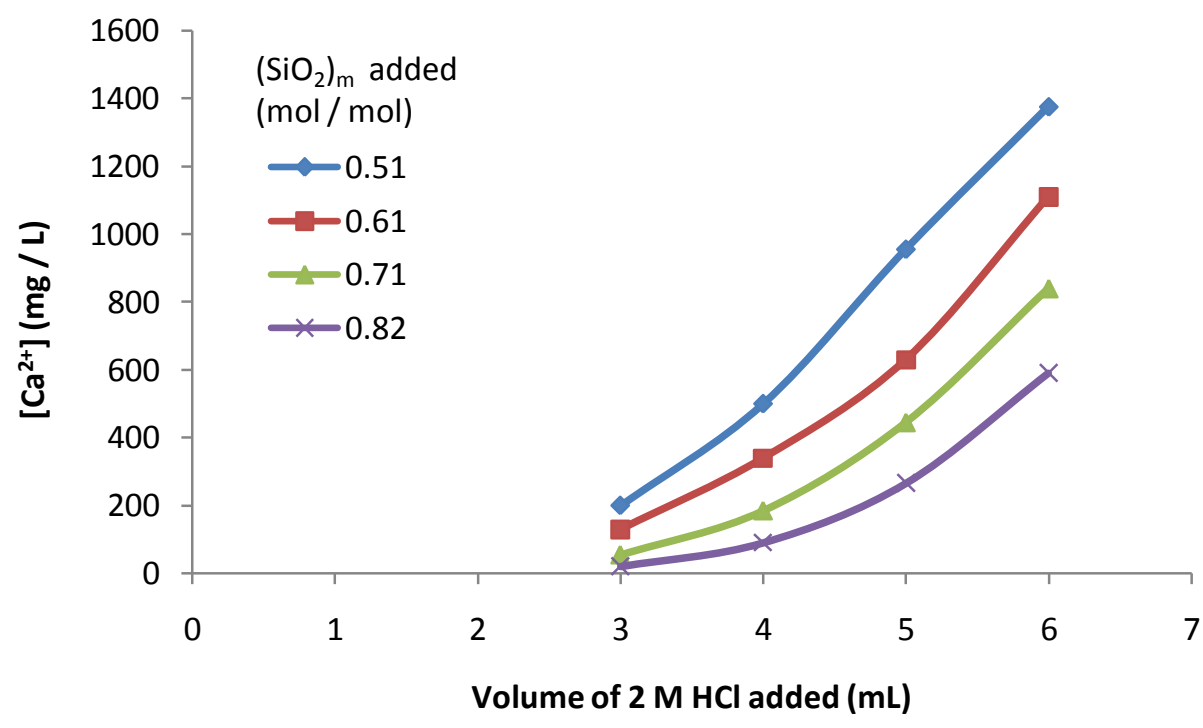

Figure 4.25: The concentration of calcium remaining in solution after reinforcement with varying amounts of monomeric silica and hydrochloric acid.

Due to the fairly insignificant effect that both the amount of monomeric silica and acid had upon the oil absorption and surface area of the materials within this range studied, other factors may be considered when determining optimum conditions for the reinforcement of nano-structured calcium silicate. The process may be designed so that the presence of a specific species in the waste water may be minimised or along economic lines where the amount of the most expensive component may be minimised.

The use of a reinforcement process significantly improved the oil absorption capacity and surface area of nano-structured calcium silicate by preventing the collapse of the structure upon removal of water inside the pores during drying (Figure 4.26). The oil absorption capacity of the material, which is measured per unit weight, cannot exceed that of the material treated with 2ethoxyethanol when maintaining the pore volume upon drying as a result of depositing additional mass onto the structure. The oil absorption capacity of reinforced material can also not exceed that achieved by treatment with 2ethoxyethanol because the additional silica added to the structure occupies a volume in the pores that would otherwise be available for absorption. One 
other effect of the reinforcement process that must also be considered for certain potential applications is the resulting modification of the surface that therefore influences any surface chemistry that may take place. The deposition of polymerised silica onto parts the structure may block the access to some of the exchangeable calcium sites on the surface and therefore reduce its capacity for ion-exchange. The blocking of these sites may also limit other forms of surface modification when attempting to functionalise the surface.

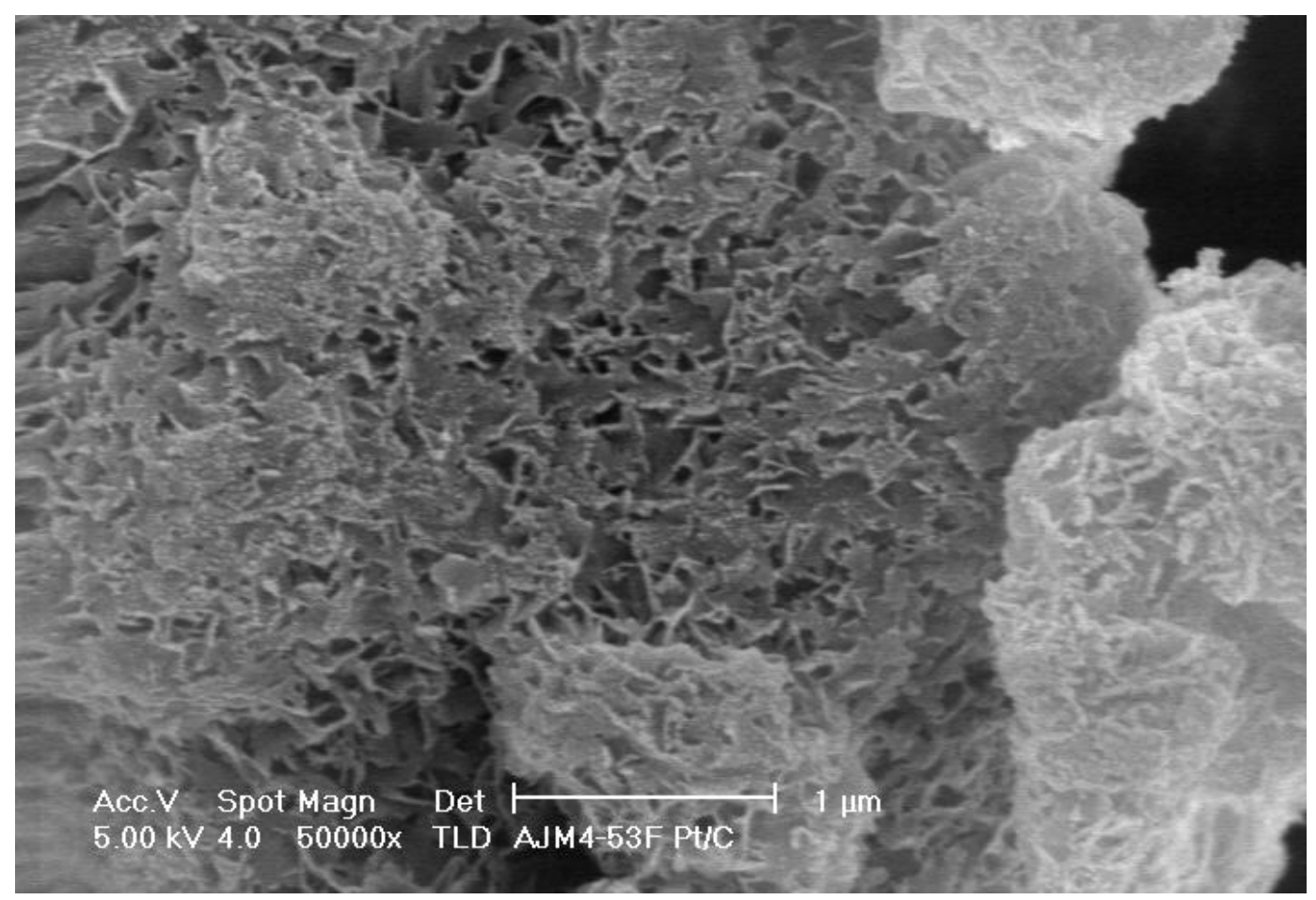

Figure 4.26: A scanning electron micrograph of nano-structured calcium silicate that has been reinforced to prevent collapse of the structure when removing water from within the pores upon drying at $110^{\circ} \mathrm{C}$.

While the reinforced material is not able to possess a pore volume as large as the material treated with 2-ethoxyethanol, it does offer other advantages. Due to the permanent added strength to the structure, reinforced nano-structured calcium silicate maintains its pore volume when water is removed from within its pores. This means that unlike the 2-ethoxyethanol treated material, the reinforced material does not collapse over time when exposed to humid environments that vary over time. As flammable organic solvents such as 2ethoxyethanol require greater precautions for their safe use and disposal, the use of water as the only solvent is also advantageous for large scale commercial production. The reinforcement process also makes the material more physically 
robust and therefore the structure is less susceptible to collapsing due to other forces that may be encountered in certain production processes.

\subsection{Effect of lowering the pH}

One of the potential applications of nano-structured calcium silicate is as a paper filler or part of a coating formulation for paper. However, it has been found that alkalinity of calcium carbonate when used as a paper filler, results in the "alkaline darkening" of groundwood pulps due to a reaction with the lignin that is still bound to the pulp (Figure 4.27). Due to the $\mathrm{pH}$ used for the formation of nano-structured calcium silicate with optimised porosity also being alkaline (ca. pH 12) it is likely that this will also cause alkaline darkening if used as a filler for paper grades that contains a large proportion of groundwood.

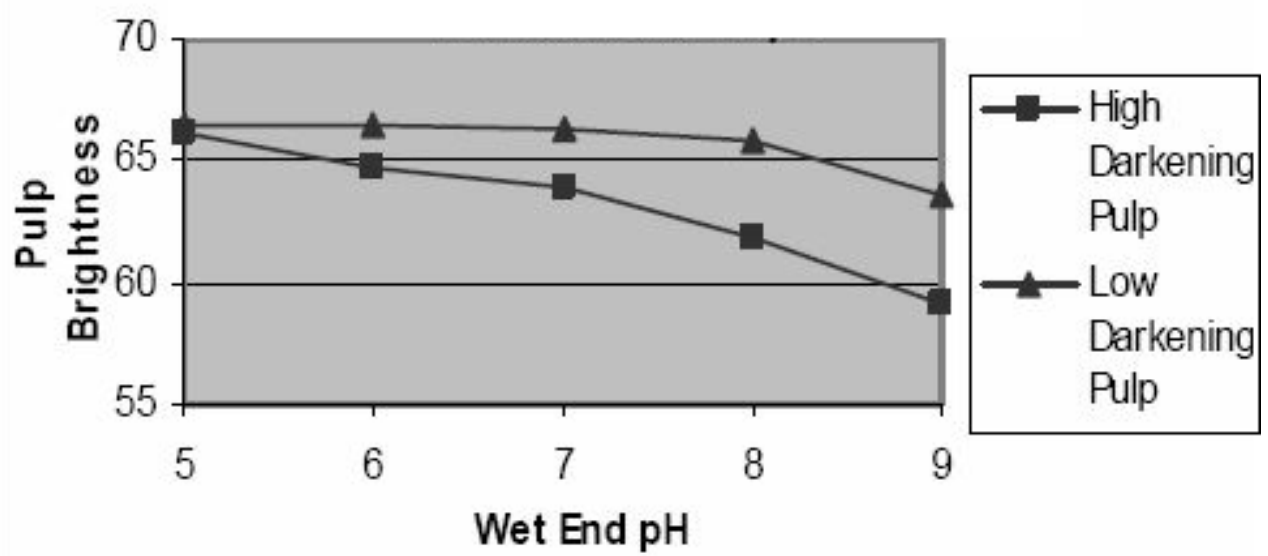

Figure 4.27: Alkaline darkening range of typical groundwood pulps over different $\mathrm{pH}$ values of the papermaking process when measured at the wet-end, that is, before water removal from the pulp (Evans and Slozer, 2003).

This is a limited problem as the majority of pulping processes currently used are alkaline rather than acidic or neutral, as the Kraft process that uses chemical pulping and therefore removes most of the lignin dominates. Alkaline processes are favoured because the chemical pulping preserves longer fibres that produce stronger sheets. These sheets are also able to be filled with lowcost calcium carbonates fillers, therefore providing an economic advantage. In North America more than $80 \%$ of fillers used by weight are calcium carbonate (Figure 4.28). Calcium carbonate is predominantly used in alkaline and neutral 
processes as the filler dissolves in acidic conditions, although modified processes can enable calcium carbonates to withstand weakly acidic conditions. Calcium carbonate fillers are generally classed as either ground calcium carbonates (GCC) that are the product of mechanically grinding mineral calcium carbonates, or precipitated calcium carbonates (PCC) that allow superior control over the particle size, shape, and purity.

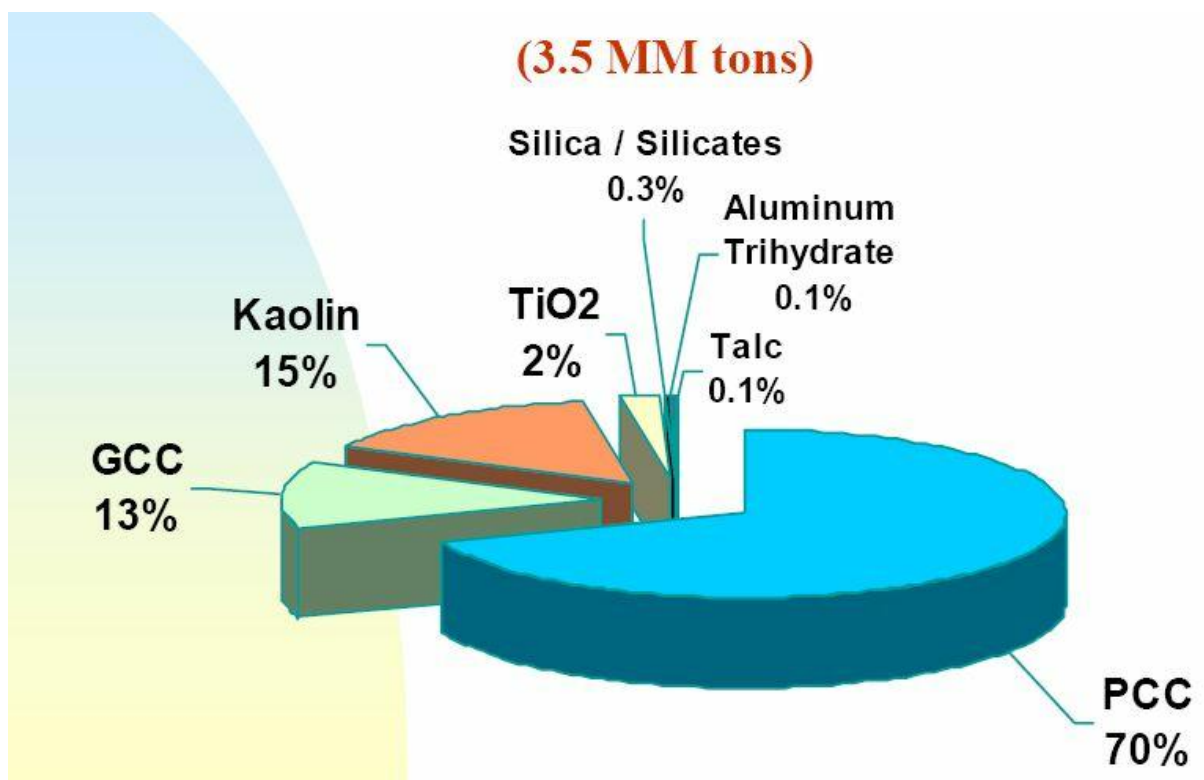

Figure 4.28: Filler use in North America (Gill, 2005)

Newsprint, directory, and groundwood specialty grades made with a high virgin groundwood content are traditionally made in acidic conditions using clay fillers to avoid alkaline darkening. The addition of fillers is made to the pulp slurry prior to sheet formation, therefore the addition of an alkaline filler to acid processes will also affect the whole wet-end chemistry of the papermaking process. As coating formulations are applied to the surface of partially or completely dry sheets, their addition has no effect upon the wet-end chemistry.

To prevent nano-structured calcium silicate from causing alkaline darkening, the effect of lowering the $\mathrm{pH}$ of the slurry after the structure had formed was studied. A nano-structured calcium silicate slurry was produced using the batch process with $0.58 \mathrm{~mol} / \mathrm{L}$ (34800 mg/L) monomeric silica, $0.62 \mathrm{~mol} / \mathrm{L}$ calcium ions (25000 mg/L), and $0.34 \mathrm{~mol} / \mathrm{L}$ hydrochloric acid and aged overnight. The 
$\mathrm{pH}$ was then slowly lowered by the drop wise addition of $10.54 \mathrm{~mol} / \mathrm{L}$ hydrochloric acid according to the method outlined in section 2.3.4. Care was taken to ensure that localised areas of low $\mathrm{pH}$ did not occur as the material completely dissolves at ca. $\mathrm{pH}$ 6. During the addition of the acid, the calcium silicate behaves like a buffer as the calcium in the structure is exchanged for the protons in solution, therefore after the initial decrease in $\mathrm{pH}$ following addition of the acid the pH slowly starts to increase. The slurry can take hours to reach equilibrium after the addition of acid, therefore in order that such experiments could be undertaken within a reasonable timeframe, the slurry was deemed to be at a set value when the $\mathrm{pH}$ was stable for 1 minute. From past experience, the difference in $\mathrm{pH}$ between the slurry after the drop wise addition of acid and if it was allowed to reach equilibrium overnight would be less the 0.2. Samples of the slurry were taken for analysis at the initial $\mathrm{pH}$ of 12.0 and also when the pH had been lowered to $10.0,8.5,7.0$, and 6.0. Each sample was washed only with water before being dried at $110^{\circ} \mathrm{C}$.

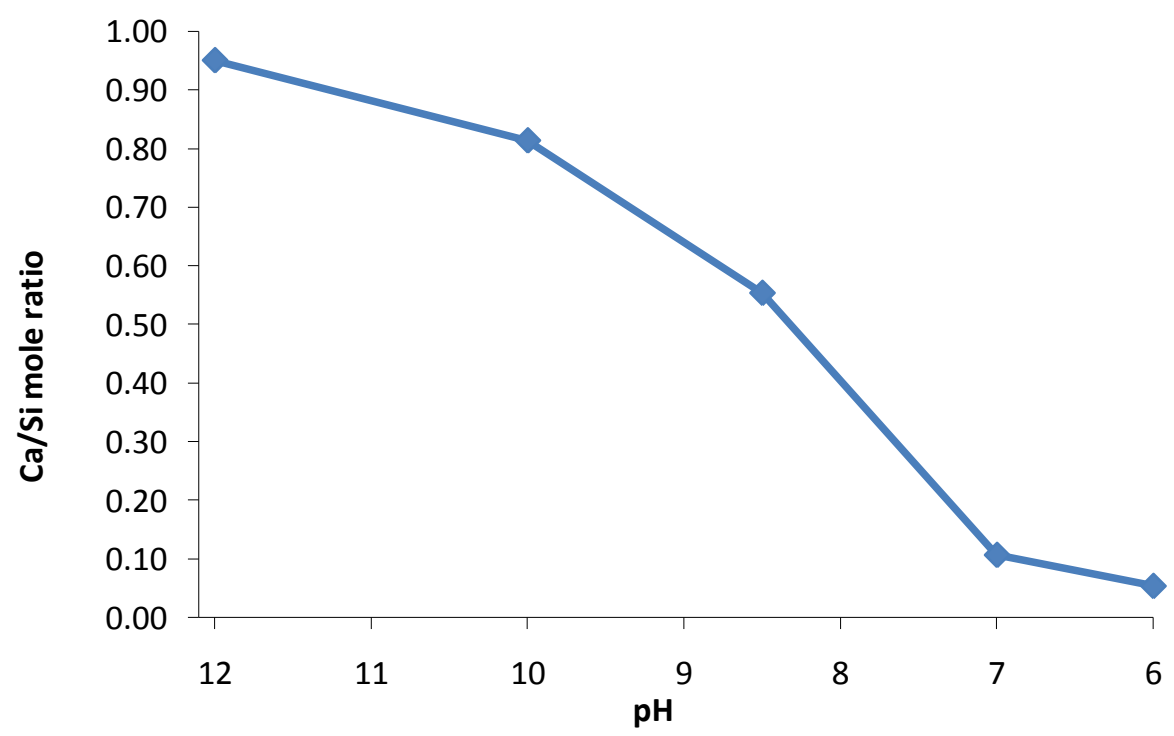

Figure 4.29: Effect of lowering the $\mathrm{pH}$ of a nano-structured calcium silicate slurry upon its resulting composition.

The effect that the acidification of nano-structured calcium silicate had upon its composition was analysed using X-ray fluorescence spectroscopy. These results showed an enormous change in the $\mathrm{Ca} / \mathrm{Si}$ mol ratio of the materials as the $\mathrm{pH}$ was lowered with the original ratio of 0.95 plummeting to 0.05 at $\mathrm{pH} 6$ (Figure 4.29). 
The large degree of calcium loss due to its exchange for protons must therefore not only occur upon the surface of the platelets, but throughout the whole structure. If the material has a similar structure to that of tobermorite (as discussed in Chapter 3), the internal calcium content of the silicate is likely to be accessible for exchange via the interlayer spacings of the structure. The platelets that make up the porous framework vary in thickness but may be estimated to be ca. $7 \mathrm{~nm}$ on average based upon SEM and TEM observations. It could therefore be estimated that the platelets are made up of only approximately three to five calcium layers flanked with silicate chains separated by interlayer spacing of $1.4 \mathrm{~nm}$. Since each platelet is made up of so few layers, the accessibility to the calcium in the structure for ion-exchange is likely to be high. As a result of the loss of calcium from the structure, a large increase in the concentration of calcium ions in solution was observed by atomic absorption spectroscopy (Figure 4.30).

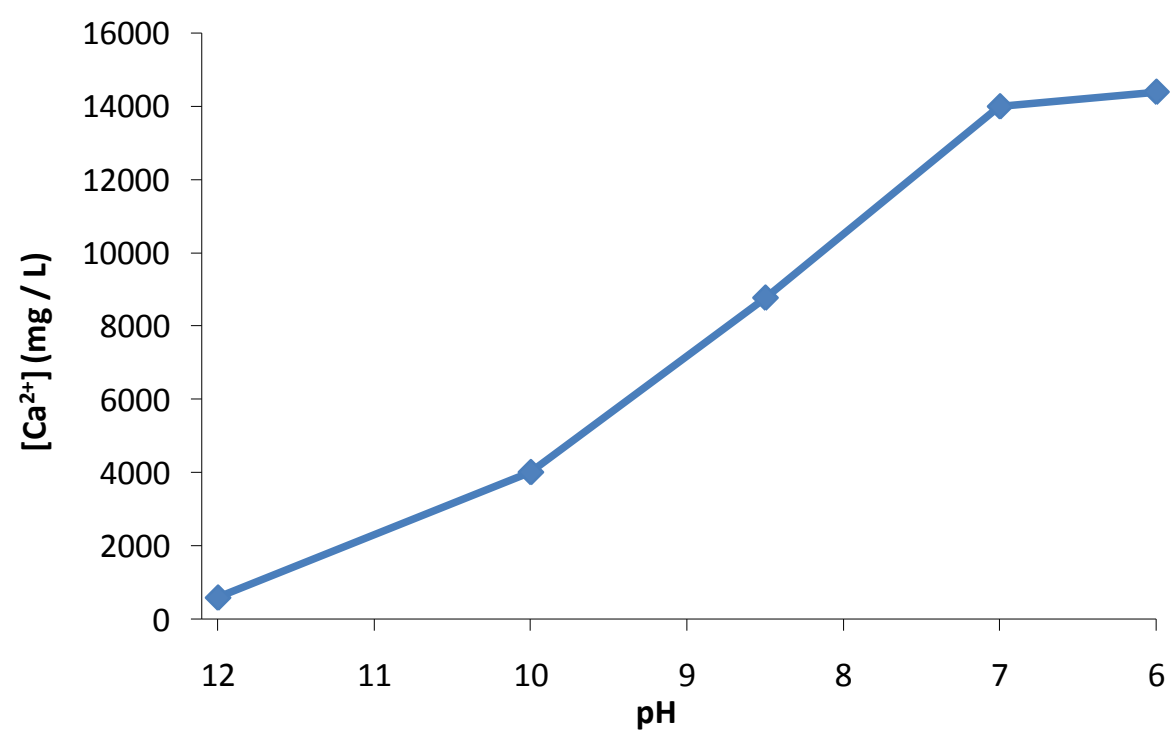

Figure 4.30: The concentration of calcium in solution as a result of acidifying a nano-structured calcium silicate slurry.

The concentration of monomeric silica in solution increases from its post reaction value of just $14.5 \mathrm{mg} / \mathrm{L}$ to the solubility of amorphous silica for the $\mathrm{pH}$ of the solution at room temperature (Figure 4.31). This suggests that a precipitated silica has formed as a result of lowering the $\mathrm{pH}$, possibly due to localised regions of low $\mathrm{pH}$ that dissolved the nano-structured calcium silicate 
and therefore released silica from the structure where it polymerised in solution.

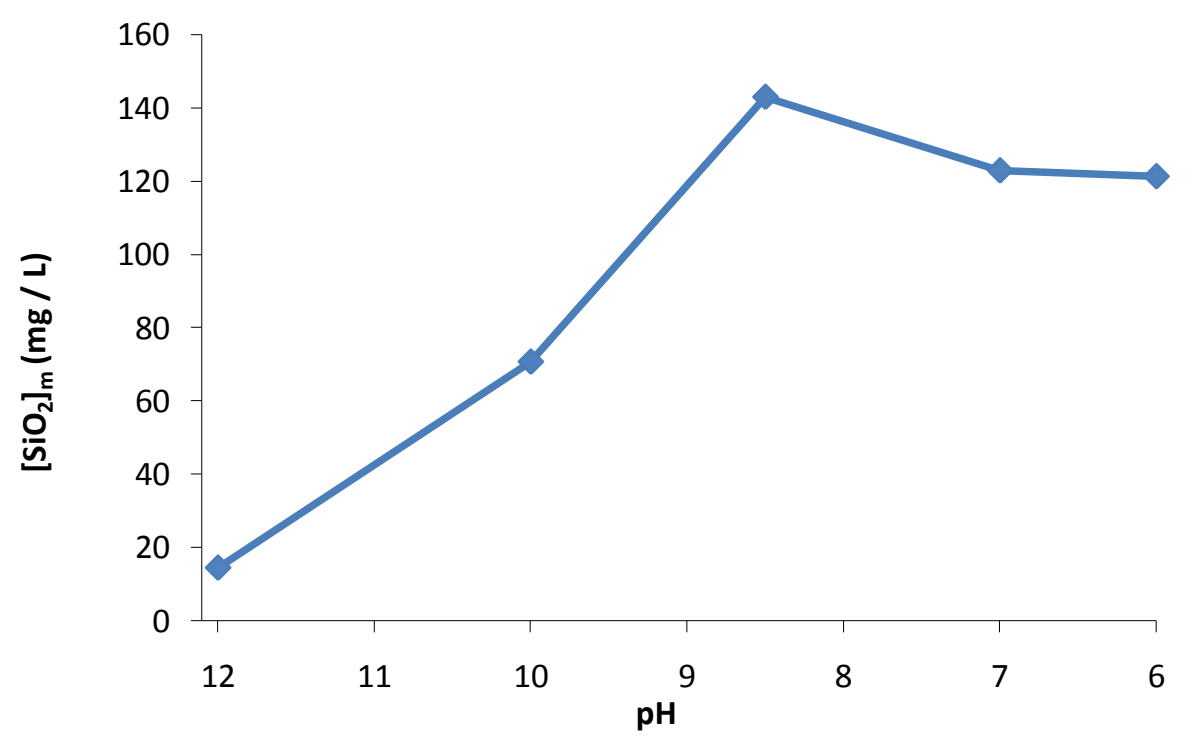

Figure 4.31: The concentration of monomeric silica in solution as a result of acidifying a nanostructured calcium silicate slurry.

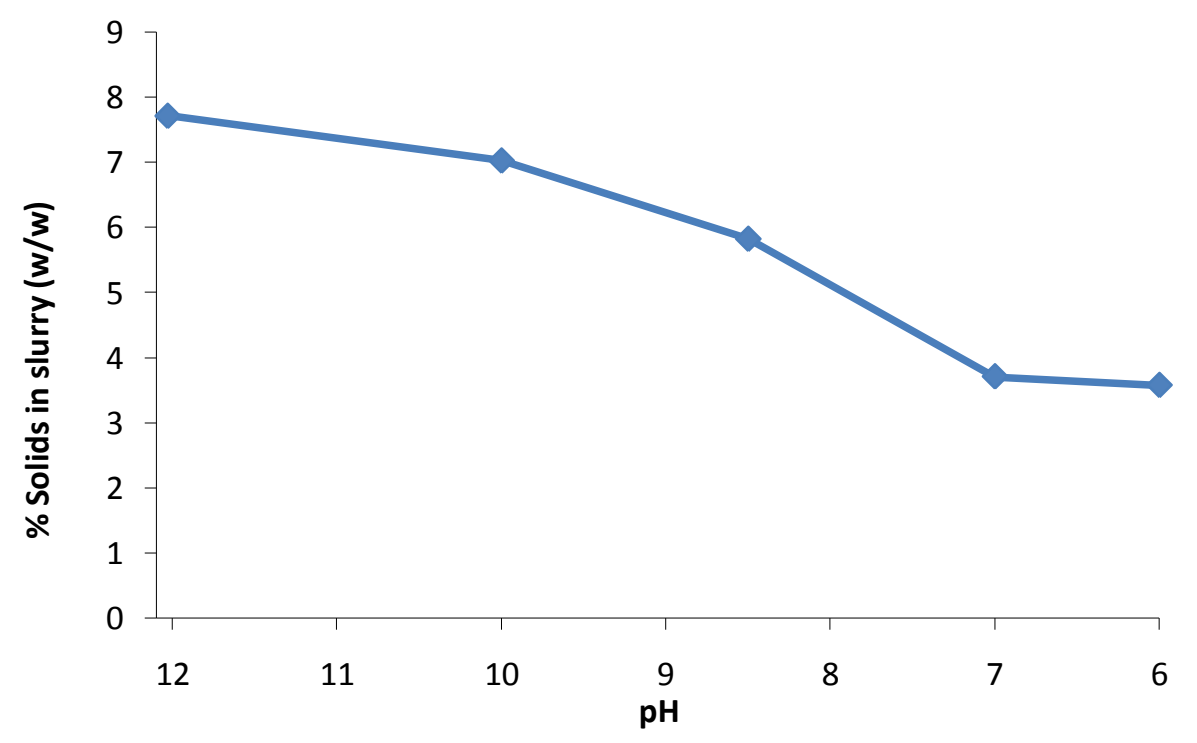

Figure 4.32: Effect of lowering the $\mathrm{pH}$ of a nano-structured calcium silicate slurry upon the $\%$ solids.

As expected from the substantial loss of calcium from the structure as the calcium content is exchanged for protons, the \% solids in the slurry (w/w, based on a dry weight at $110^{\circ} \mathrm{C}$ ) decreases as the $\mathrm{pH}$ is lowered (Figure 4.32). The total volume of acid added during the process was $7.6 \%$ of the total volume of the reaction due to the use of a highly concentrated acid and would therefore 
only decrease the \% solids by a small amount. The decrease in the solid content from $7.7 \%$ following the formation of the structure to $3.7 \%$ at $\mathrm{pH} 7$ means that approximately half of the mass is lost. This is in agreement with the change in composition that shows almost complete loss of calcium from the structure, which contributes approximately half of the materials weight. This decrease in product would have serious ramifications upon the economic viability of the process as well as possible concerns over disposal of the waste water due to the higher concentration of species present.

The lowering of the $\mathrm{pH}$ also had an effect upon the physical properties of the material. Both the specific surface area and the oil absorption capacity of the material when washed only with water increased substantially as the $\mathrm{pH}$ decreased (Figure 4.33).

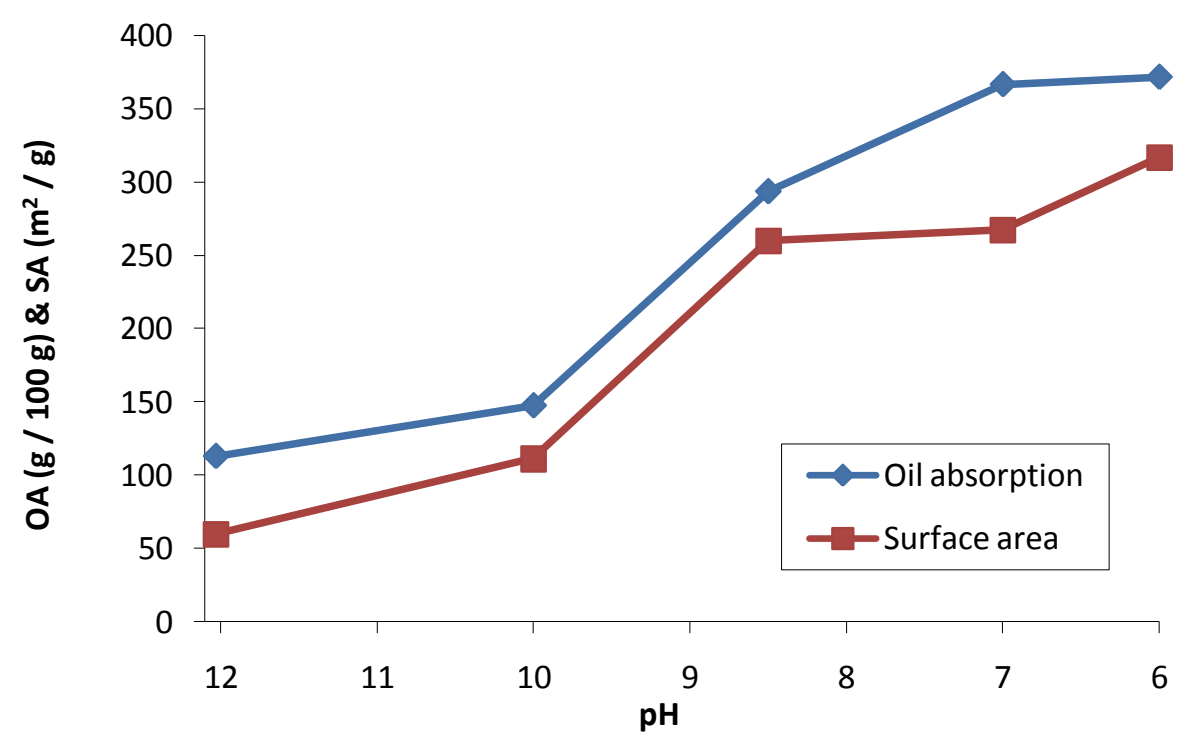

Figure 4.33: Effect of lowering the pH upon the surface area (SA) and oil absorption capacity (OA) of nano-structured calcium silicate.

The reasons for this could possibly be due to a reinforcing effect as the dissolution of silica from the structure could result in the released silica being re-deposited upon the silicate in regions of negative curvature as the lowering of the $\mathrm{pH}$ also decreases the solubility of silica in the solution. This reinforcing effect would strengthen the structure to prevent collapse when the material is dried. Another possible contributing factor in the improvement of the surface area and oil absorption capacity is that as both properties are measured per 
unit weight, the loss of mass as the calcium content decreases would therefore also result in an increase in these properties as the overall porous framework remains intact. The preservation of the framework during lowering the $\mathrm{pH}$ of the nano-structured calcium silicate slurry was somewhat unexpected after such a large proportion of calcium was removed from the structure, since more than just surface species must have been removed. Despite the substantial changes at an atomic level that must have occurred through replacement of nearly all of the calcium content with protons, the presence of the platelets framework is still visible when studying the structure of samples from the untreated initial pH of 12 to pH 7 (Figure 4.34, Figure 4.35, and Figure 4.36).

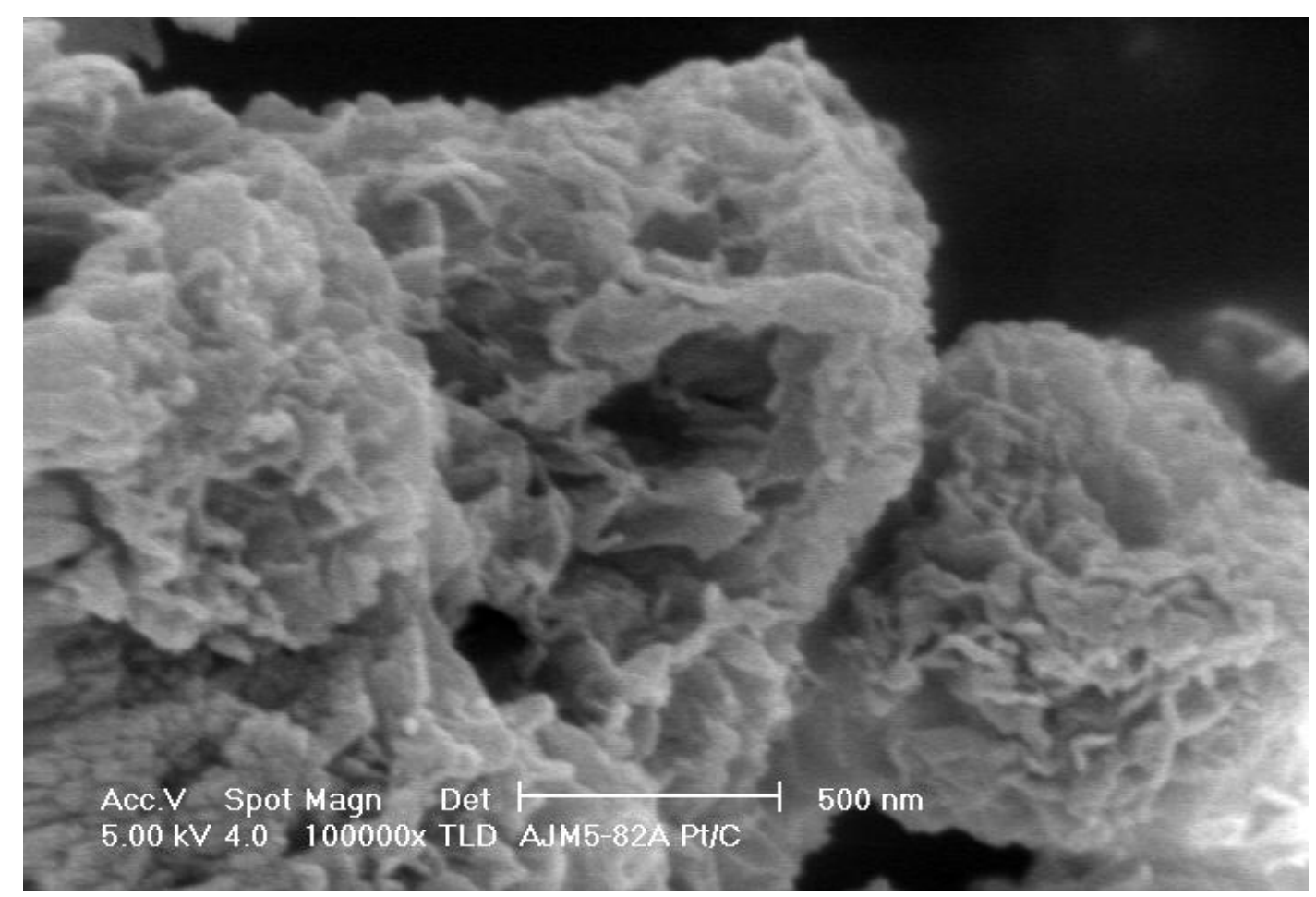

Figure 4.34: Scanning electron micrograph of base material at $\mathrm{pH} 12.0$ prior to the addition of acid. 


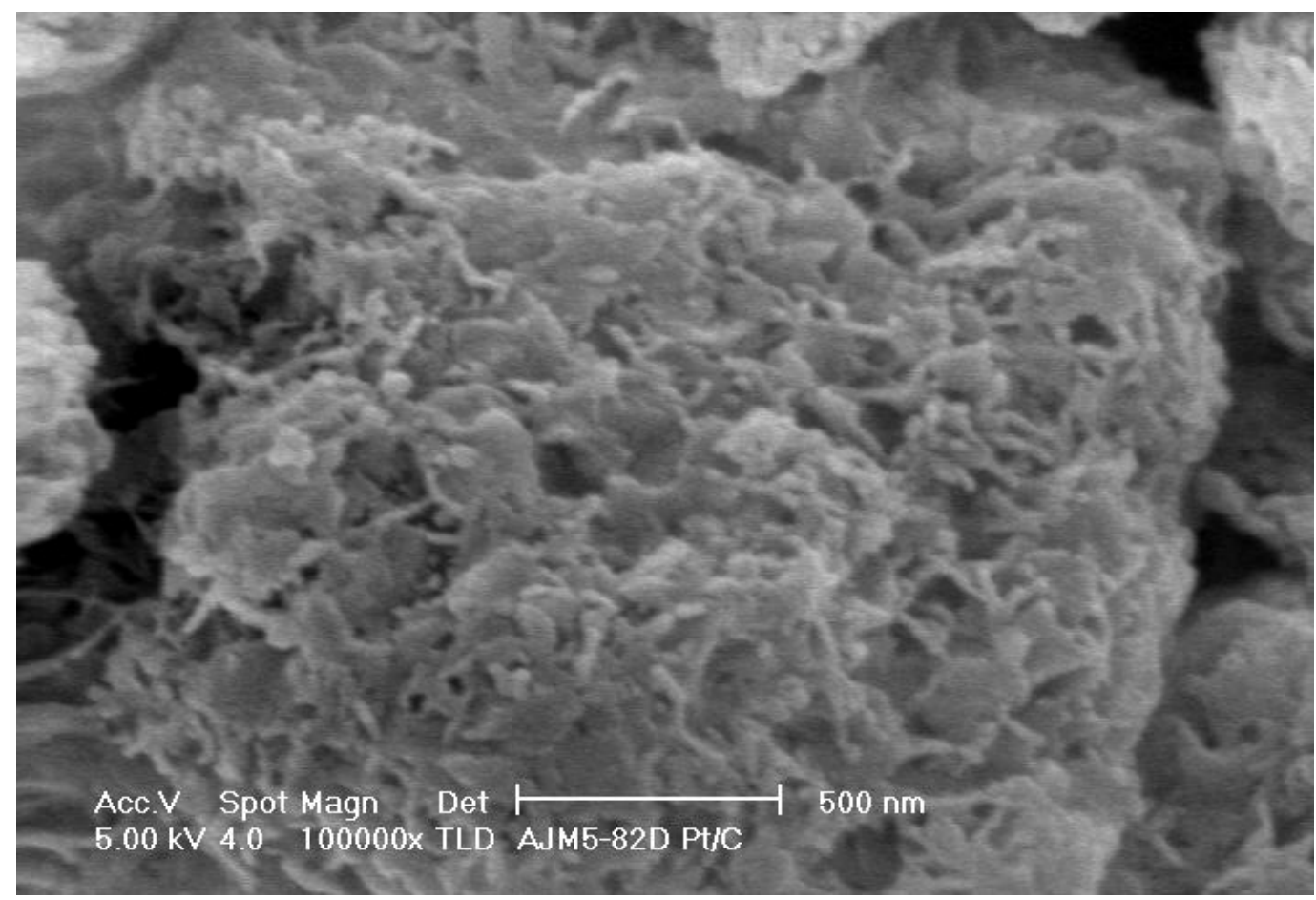

Figure 4.35: Scanning electron micrograph of nano-structured calcium silicate at pH 10.0.

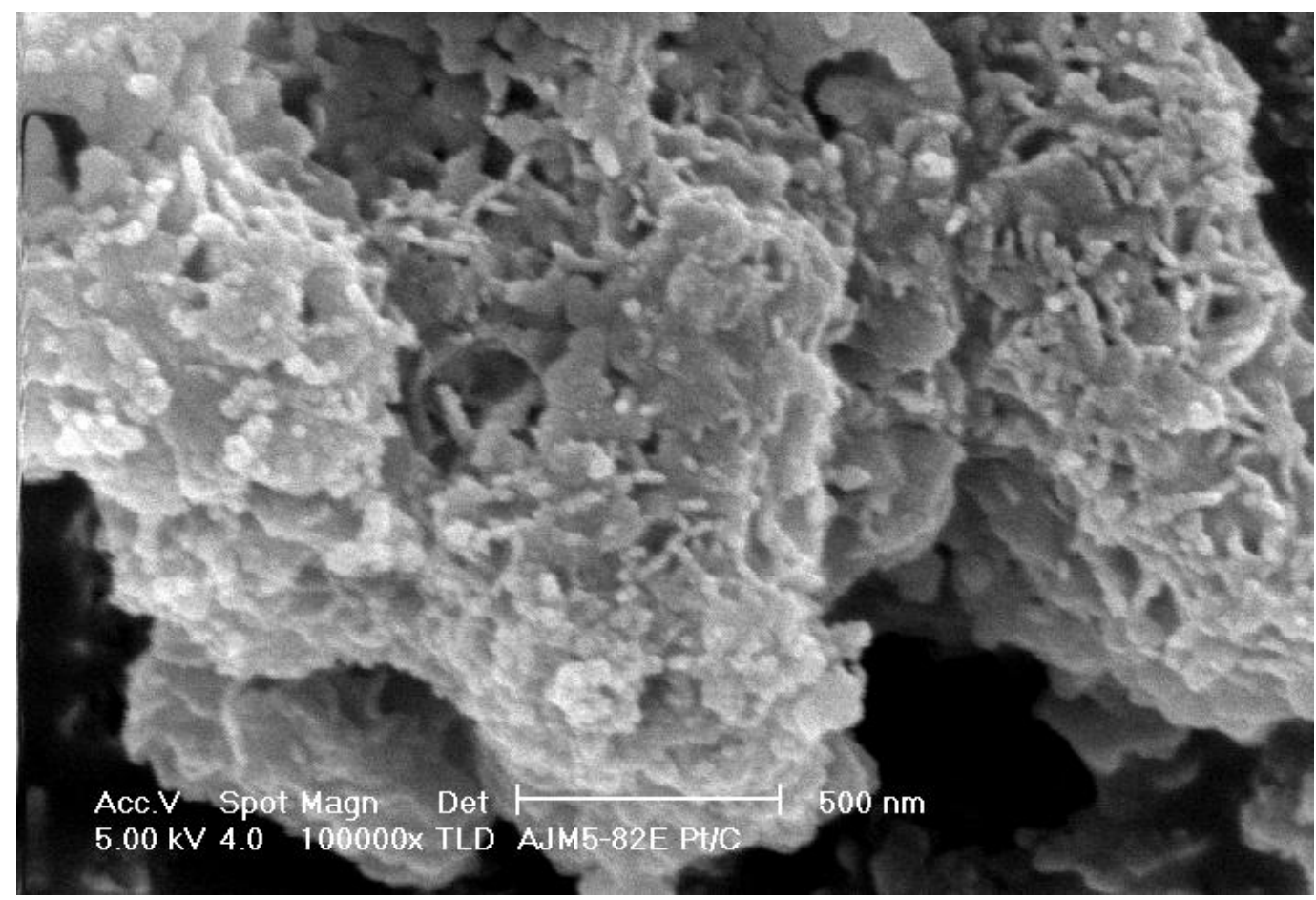

Figure 4.36: Scanning electron micrograph of nano-structured calcium silicate at $\mathrm{pH} 7.0$

As would be anticipated, the removal of such a large proportion of the calcium from the structure has a noticeable effect upon the powder X-ray diffraction pattern, which differs greatly from the untreated material (Figure 4.37). The broad peak from 15 to $30^{\circ} 2 \theta$ is typical for that observed for amorphous silica (Himmel et al., 1985) and may be attributed to the formation of independent 
silica particles polymerised at the lower $\mathrm{pH}$ as the solubility is decreased and as the structure is partially dissolved. Another explanation could be that the peak is due to the remaining silicate that would have lost all of its long-range order at pH 7.0. Sharp peaks due to the presence of a calcium carbonate impurity are observed (PDF: 05-0586) and the broad peaks due to calcium silicate hydrate are not visible.

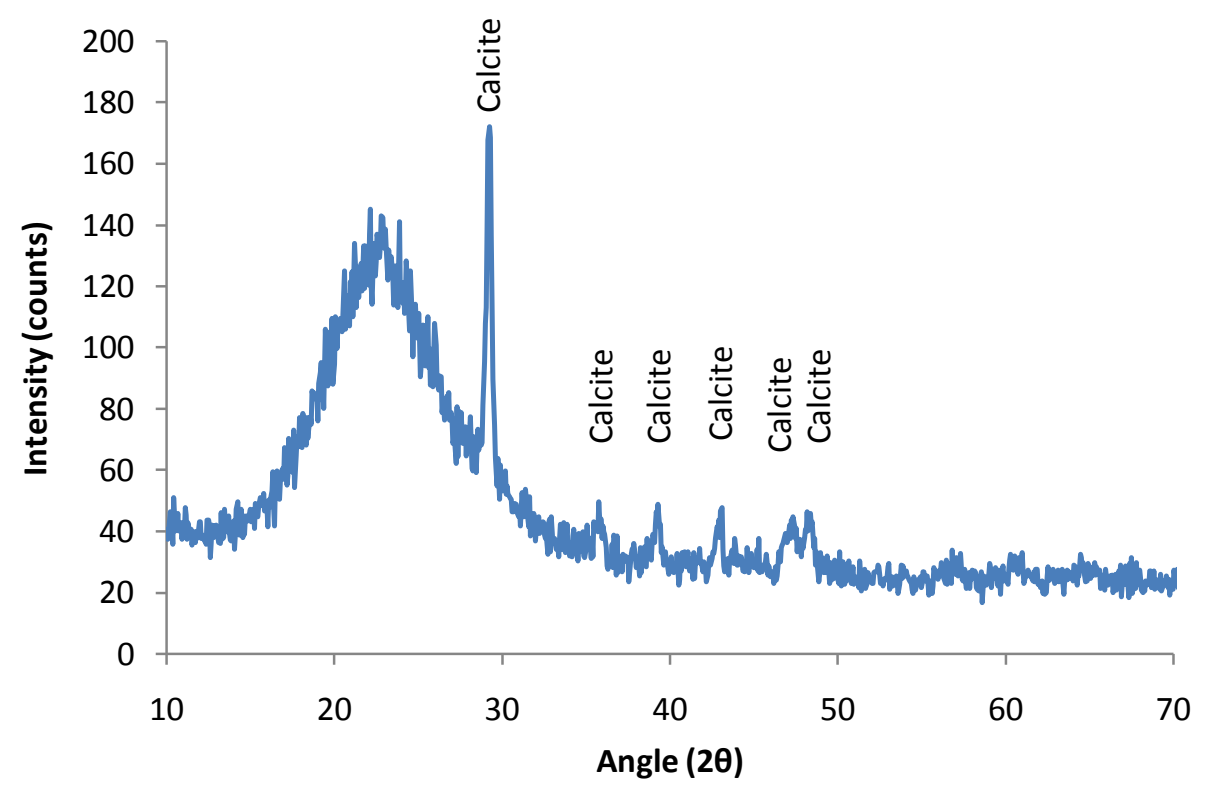

Figure 4.37: Powder X-ray diffractogram of nano-structured calcium silicate adjusted to pH 7.0.

Thermogravimetric analysis of the samples that were $\mathrm{pH}$ adjusted show that there is little difference between the untreated sample at pH 11.6 and the same material adjusted to pH 9.0 (Figure 4.38). Both materials lose an identical large amount of weight (ca. $12.5 \%$ ) up to $250{ }^{\circ} \mathrm{C}$. This weight loss is likely to be due to the loss of loosely bound water as Shaw et al. (2000a) described for the analysis of tobermorite over a 50 to $250{ }^{\circ} \mathrm{C}$ range. The sample that was adjusted to $\mathrm{pH} 7.0$ loses only ca. 7.5\% weight over this temperature range. The reduction in weight loss is likely to be a result of the removal of a large proportion of calcium from the structure. The loss of calcium would cause the loss of some water in the interlayer spacing that is co-ordinated to the structure over the calcium layers in the untreated material. The effect is probably competing against the increase in surface area observed as the materials become less alkaline (Figure 4.33) and the materials therefore possessing more surface sites for water to bind to. Hence the reason why the loss of calcium does not cause 
the material at pH 9.0 to lose less weight than the material at pH 11.6 could be the difference in surface areas of $200 \mathrm{~m}^{2} / \mathrm{g}$ compared with $60 \mathrm{~m}^{2} / \mathrm{g}$ respectively. Both materials at pH 9.0 and pH 7.0 have similar surface areas, but differ greatly in the amount of calcium remaining in the structure, therefore leading to the difference in weight lost over the 50 to $250{ }^{\circ} \mathrm{C}$ temperature range.

The other significant loss of weight for all of the samples occurs over a 450 to $680^{\circ} \mathrm{C}$ range. While the tobermorite sample that Shaw analysed was anomalous in that it did not contain any hydroxyl groups, a hillebrandite [ $\left.\mathrm{CaSiO}_{3}(\mathrm{OH})_{2}\right]$ sample that was analysed had a main weight change over the temperature ranges of 480 to $570{ }^{\circ} \mathrm{C}$ and 570 to $670{ }^{\circ} \mathrm{C}$. This weight loss was attributed to the loss of structural hydroxyl species. All three nano-structured calcium silicates lost a similar weight (ca. 7\%) over this range indicating that they all have similar proportions of structural hydroxyl groups.

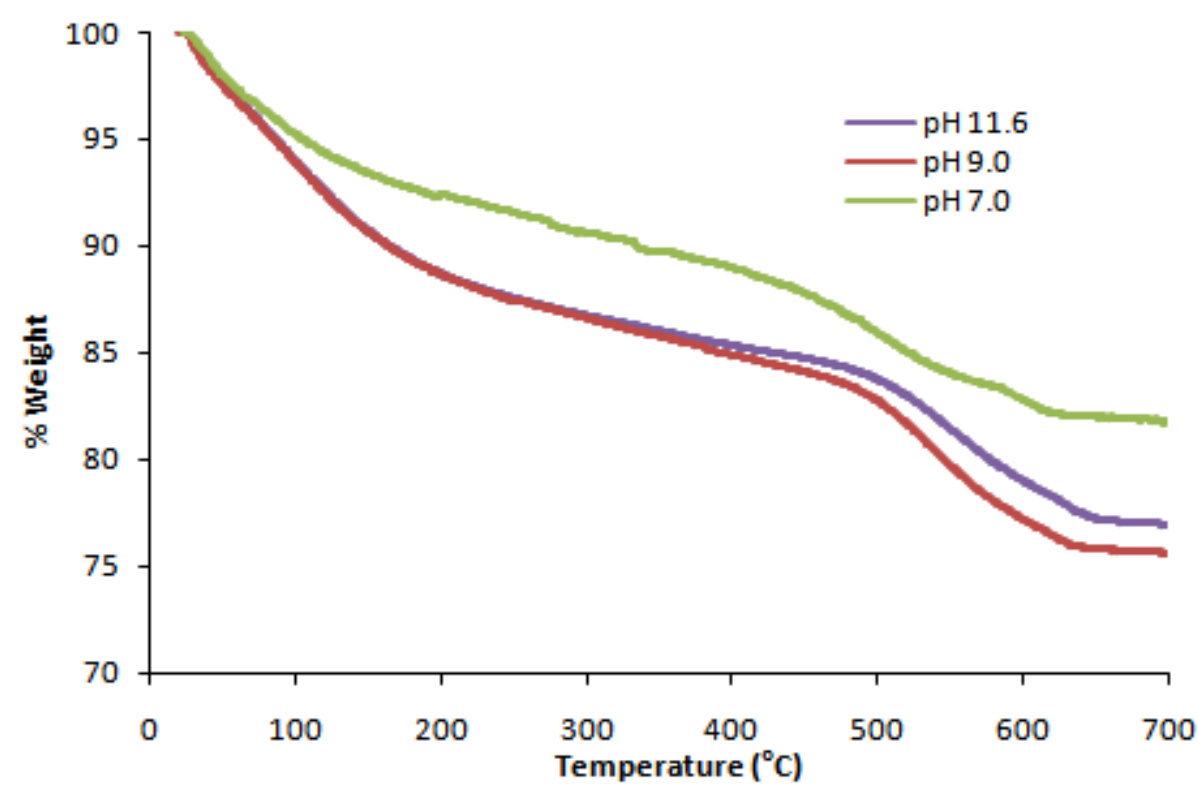

Figure 4.38: Thermal analysis curves of nano-structured calcium silicate with the $\mathrm{pH}$ altered post formation.

As the purpose of this study is to produce material suitable for use as a paper filler or in a paper coating, the effect that this process has upon the optical properties of the material must also be considered. Technical grade calcium hydroxide was used for precipitating the material, with the same method used for its formation as described for the previous example. As shown in Figure 
4.39 , the lowering of the $\mathrm{pH}$ has little effect upon either the whiteness or brightness of the material with a slight improvement measured for the sample at $\mathrm{pH}$ 7. The lowering of the $\mathrm{pH}$ also had no significant effect upon the $\mathrm{a}^{*}$ value measured (the red-green axis), although the change in the $b^{*}$ value indicates a change from a yellow hue to a blue hue - which to the human eye makes the material appear more white (Figure 4.40).

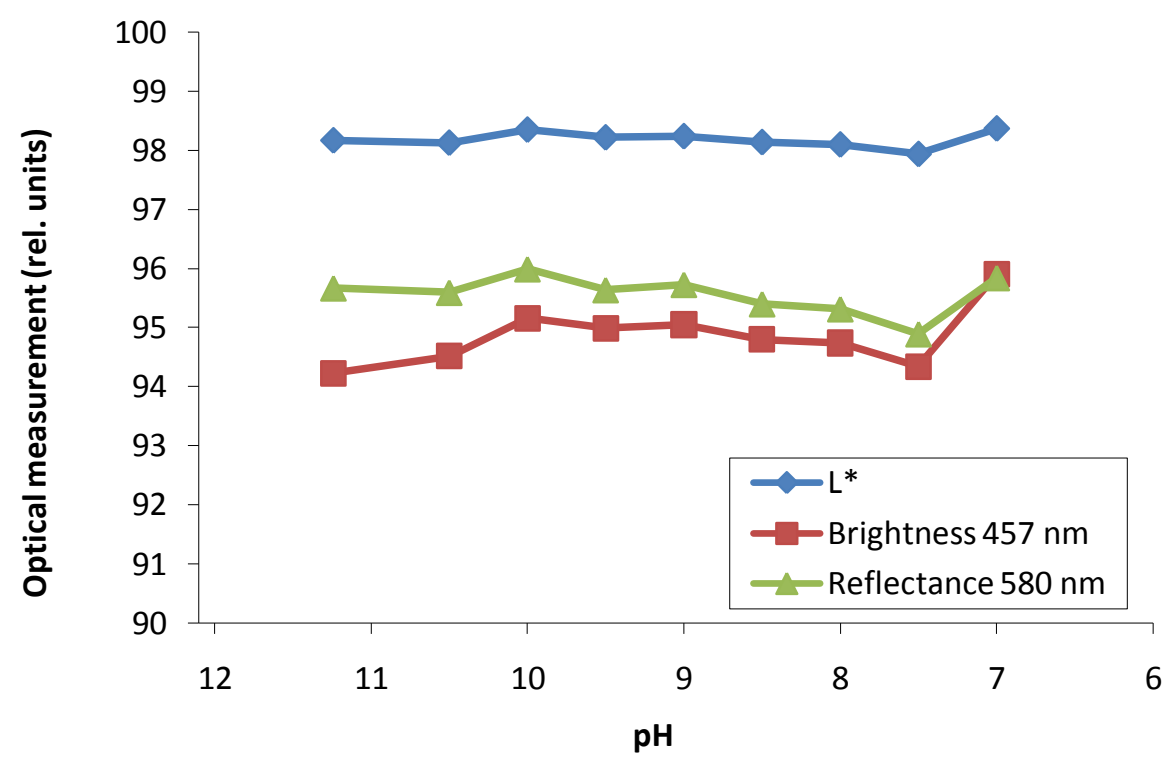

Figure 4.39: Effect of lowering the $\mathrm{pH}$ of a nano-structured calcium silicate upon the whiteness and brightness of the material (relative units).

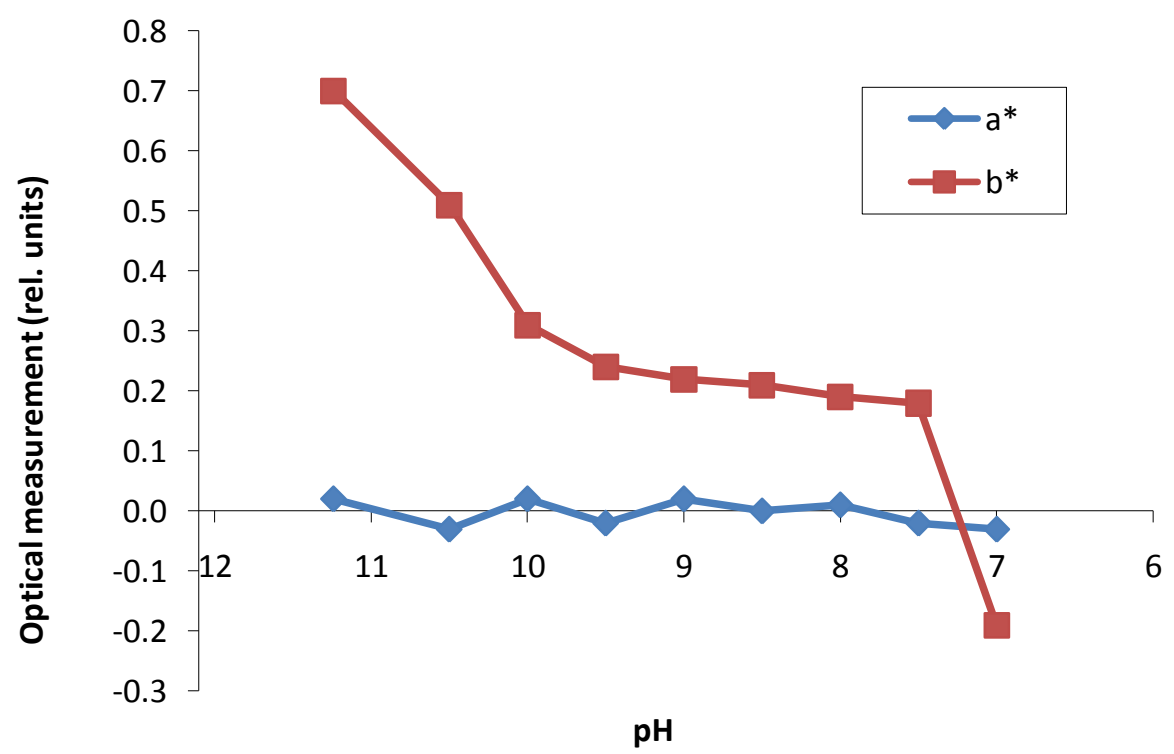

Figure 4.40: Effect of lowering the $\mathrm{pH}$ of nano-structured calcium silicate upon the colour of the material (relative units). 
Although the improvement in the material's physical and optical properties upon decreasing the $\mathrm{pH}$ is advantageous, the economic aspect of adding calcium to form the initial structure only to wash it away later greatly negates any benefits of the process. To this end, the possibility of recycling the calcium in the filtrate from the material adjusted to $\mathrm{pH} 7.0$ was investigated. If the calcium that is lost from the structure can be recovered and used for further reactions, the economic viability of the process would be improved. A method was therefore devised whereby the filtrate was collected after filtering a material that had been adjusted to pH 7.0 before any plug flows of water are washed through the filter-cake, so that there is no variation due to dilution. For example, a $500 \mathrm{~mL}$ reaction of $0.58 \mathrm{~mol} / \mathrm{L}$ monomeric silica and $0.62 \mathrm{~mol} / \mathrm{L}$ calcium ions with $0.34 \mathrm{~mol} / \mathrm{l}$ hydrochloric acid formed the base material. The nano-structured calcium silicate slurry was adjusted to $\mathrm{pH} 7.0$ and left overnight to reach equilibrium. The $\mathrm{pH}$ had increased to $\mathrm{pH} 8.0$ so further acid was added to return the slurry to $\mathrm{pH}$ 7.0. In total, $50 \mathrm{~mL}$ of $10.54 \mathrm{~mol} / \mathrm{L}$ hydrochloric acid was added to lower the $\mathrm{pH}$ to 7.0. When the product was filtered, $325 \mathrm{~mL}$ of filtrate was collected that had a calcium concentration of $21000 \mathrm{mg} / \mathrm{L}(0.52 \mathrm{~mol} / \mathrm{L})$. To obtain the full initial starting concentration of $0.62 \mathrm{~mol} / \mathrm{L}$ of calcium for the following $500 \mathrm{~mL}$ reaction using the recycled filtrate, additional calcium hydroxide would need to be added. The filtrate provides $0.16 \mathrm{~mol}$ calcium and the addition of $15.1 \mathrm{~g}$ calcium hydroxide contributes $0.20 \mathrm{~mol}$ to make the $0.31 \mathrm{~mol}$ required. The calcium hydroxide was added to the filtrate and 60.0 g sodium silicate $\left(29 \% \mathrm{SiO}_{2}\right)$ was diluted to $175 \mathrm{~mL}$ with water (that would give a concentration of $0.58 \mathrm{~mol} / \mathrm{L}$ for the total slurry when the reactants were mixed). As the $\mathrm{pH} 7$ filtrate lowers the $\mathrm{pH}$ of the reaction, no additional acid was required to adjust the $\mathrm{pH}$ into the optimal range. The reaction was then carried out according the standard batch process. The development of the structure from the reaction using the recycled filtrate was monitored using 2-ethoxyethanol to prevent collapse of the structure upon drying and the ageing process was found to take place as per a standard reaction (Figure 4.41). 


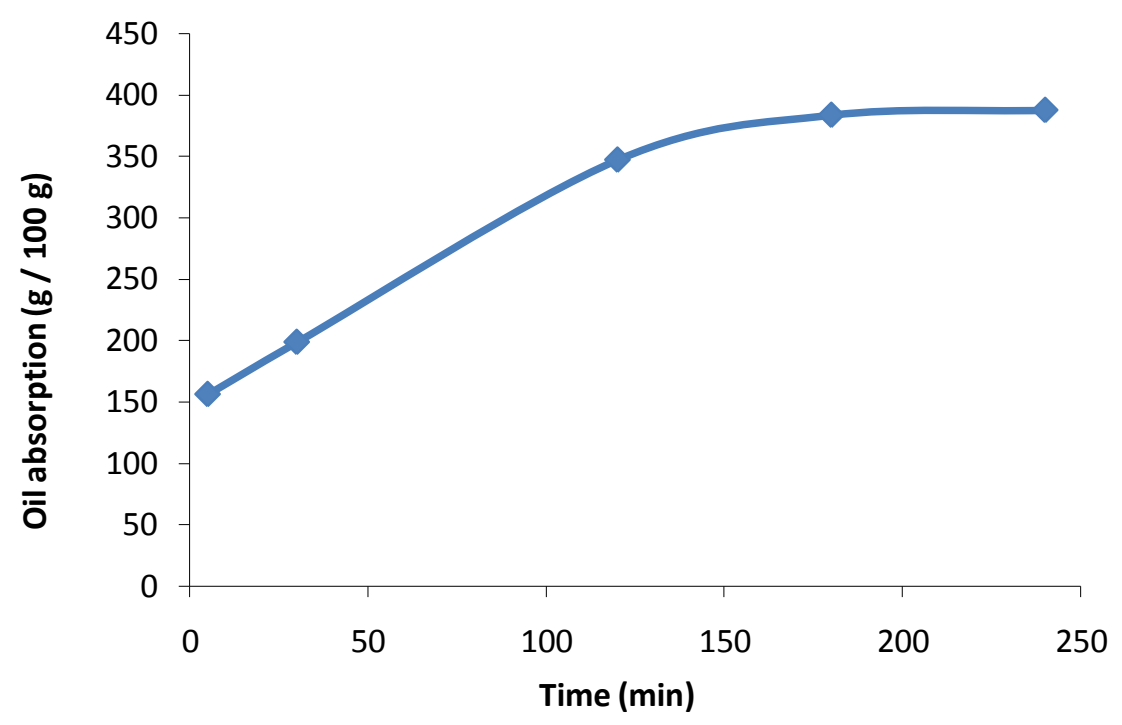

Figure 4.41: Development of the oil absorption capacity of 2-ethoxyethanol washed nanostructured calcium silicate formed using recycled filtrate.

The slurry formed from the reaction using the recycled filtrate as the source of a proportion of the calcium content was then itself adjusted to $\mathrm{pH} 7$ with samples taken at regular intervals of $\mathrm{pH}$. The lowering of the $\mathrm{pH}$ had approximately the same effect upon the oil absorption capacities and surface areas using the recycled filtrate (Figure 4.42) as for using only calcium hydroxide as the calcium source (as per the first example in Figure 4.33).

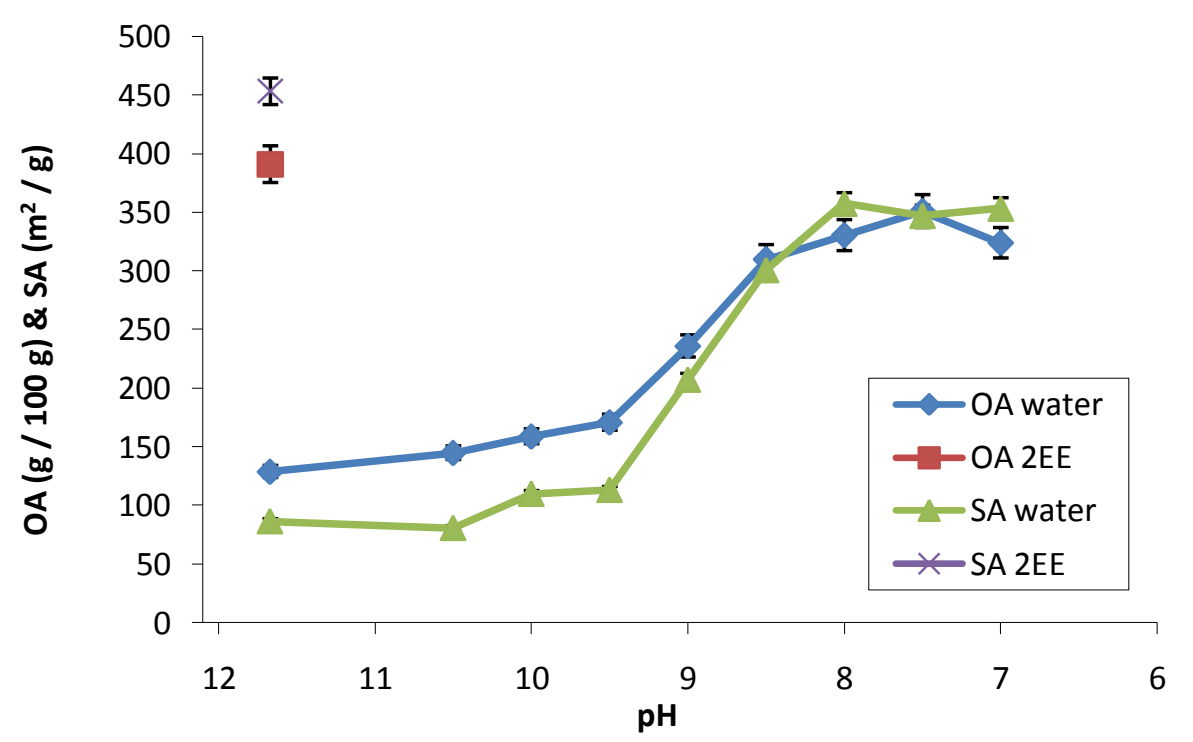

Figure 4.42: The effect of lowering the $\mathrm{pH}$ of a slurry formed using recycled filtrate upon the surface area and oil absorption capacity. 
These results suggest that there is no significant difference between using the combination of recycled filtrate and calcium hydroxide or solely calcium hydroxide as the source of calcium for the reaction. The structure of the material at pH 7.5 also appears to be the same as those produced using exclusively calcium hydroxide (Figure 4.43).

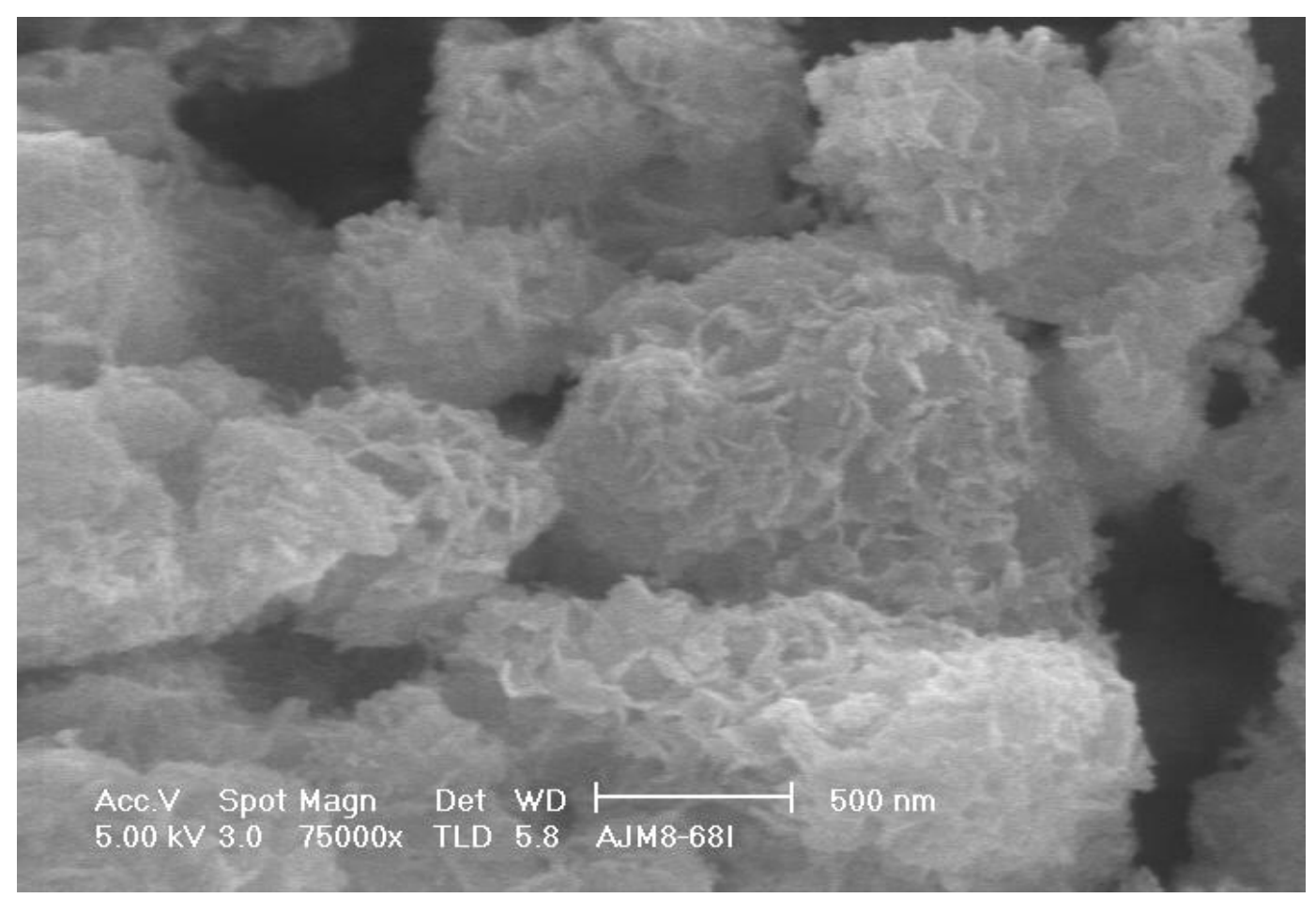

Figure 4.43: Scanning electron micrograph of nano-structured calcium silicate formed with recycled filtrate and adjusted to $\mathrm{pH} 7.5$.

A series of experiments was performed using the method described above whereby the filtrate was recycled on four occasions. The $\mathrm{pH}$ for each cycle was adjusted to $\mathrm{pH} 7.5$ rather than $\mathrm{pH} 7.0$ as in the previous example. As less calcium is removed from the structure with the reduced amount of acid added, the resulting difference in the calcium concentration of the filtrate is taken into account by interpolating the data in Figure 4.30. A summary of the oil absorption capacities for each cycle of the process is given in Table 4.5. Reasonably consistent results were obtained by continually recycling the filtrate, which enhances the applicability of the process. The number of cycles needs to be limited however, as sodium and chloride spectator ions from the sodium silicate and hydrochloric acid become increasingly concentrated with each reaction. 
Table 4.5: Oil absorption capacities of materials formed by continually recycling the filtrate (g/100 g).

\begin{tabular}{lcccc}
\hline & \multicolumn{2}{c}{$\begin{array}{c}\text { 2EE washed } \\
(\mathrm{g} / 100 \mathrm{~g})\end{array}$} & \multicolumn{2}{c}{$\mathrm{pH} \mathrm{7.5}$} \\
& $(\mathrm{g} / 100 \mathrm{~g})$ \\
\hline Original material & 420 & \pm 20 & 300 & \pm 10 \\
1st cycle & 370 & \pm 20 & 265 & \pm 10 \\
2nd cycle & 405 & \pm 20 & 275 & \pm 10 \\
3rd cycle & 405 & \pm 20 & 270 & \pm 10 \\
4th cycle & 415 & \pm 20 & 260 & \pm 10 \\
\hline
\end{tabular}

The $\mathrm{pH}$ adjustment of nano-structured calcium silicate may be necessary when the highly alkaline $\mathrm{pH}$ of the material prepared using standard conditions is not suitable for certain applications, such as filling paper sheets that contain a high proportion of mechanical pulp. By lowering the $\mathrm{pH}$ to neutral or near-neutral conditions the physical and optical properties were improved above those of the unmodified material simply washed with water. The feasibility of the process from an economic stand point is questionable due to the large loss of product mass that occurs during the process as the majority of calcium in the structure is exchanged for protons, therefore increasing the cost per tonne of material. The ability to recycle the filtrate and therefore recover a portion of the dissolved calcium from the structure goes some way to addressing this issue. It was found that the filtrate could be repeatedly recycled and that product formed by use of the recycled filtrate was equivalent to that formed using the orthodox method with calcium hydroxide. The loss of calcium from the structure will reduce the ability to functionalise the material or use the material for ion-exchange as surface protons are not as readily exchanged as the surface calcium. The superior oil absorption capacity of the reinforced material (and the more beneficial economics of that process) means that neutral or nearneutral nano-structured calcium silicate would only likely be applied in specialised applications. 


\section{Chapter 5}

\section{OPTIMISATION OF REACTION CONDITIONS}

\subsection{Changing the calcium to silicon ratio}

X-ray diffractograms of nano-structured calcium silicate (Figure 3.13) suggest that the long-range atomic-level structure may be related to that of other disordered calcium silicate hydrates and therefore also the crystalline mineral tobermorite. All of these compounds are known to be able to accommodate variations of the $\mathrm{Ca} / \mathrm{Si}$ ratio, primarily due the disorder that is inherent in their structures and the ability to accommodate additional calcium in the interlayer cavities. The ability of nano-structured calcium silicate to form with differing $\mathrm{Ca} / \mathrm{Si}$ ratios while still maintaining the physical properties of a large pore volume and a high surface area was therefore of interest. This is of particular significance for the ability of increasing the scale of the process to meet potential commercial demand as this would increase the operating window through which production could occur as the properties of the final product would not be dependent upon the level of fine control more readily achieved at a bench-top scale. Two series of experiments were carried out based on an initial Ca/Si ratio of 0.98 by using $0.32 \mathrm{~mol} / \mathrm{L}$ monomeric silica and $0.31 \mathrm{~mol} / \mathrm{L}$ calcium hydroxide with $0.17 \mathrm{~mol} / \mathrm{L}$ hydrochloric acid for controlling the $\mathrm{pH}$ of the reaction. In one series of experiments the calcium and hydrochloric acid concentrations were progressively decreased in 5\% increments while maintaining the same monomeric silica concentration, while in the other series the monomeric silica concentration was decreased in 5\% increments while keeping the calcium and hydrochloric acid concentrations fixed. Each sample produced using a different $\mathrm{Ca} / \mathrm{Si}$ ratio was allowed to age and was then treated 
with 2-ethoxyethanol to prevent collapse of the structure before drying at 110 ${ }^{\circ} \mathrm{C}$. The measured change in the oil absorption capacity with $\mathrm{Ca} / \mathrm{Si}$ ratio gives a broad curve that reaches a maximum at a ratio of 1.1 (Figure 5.1).

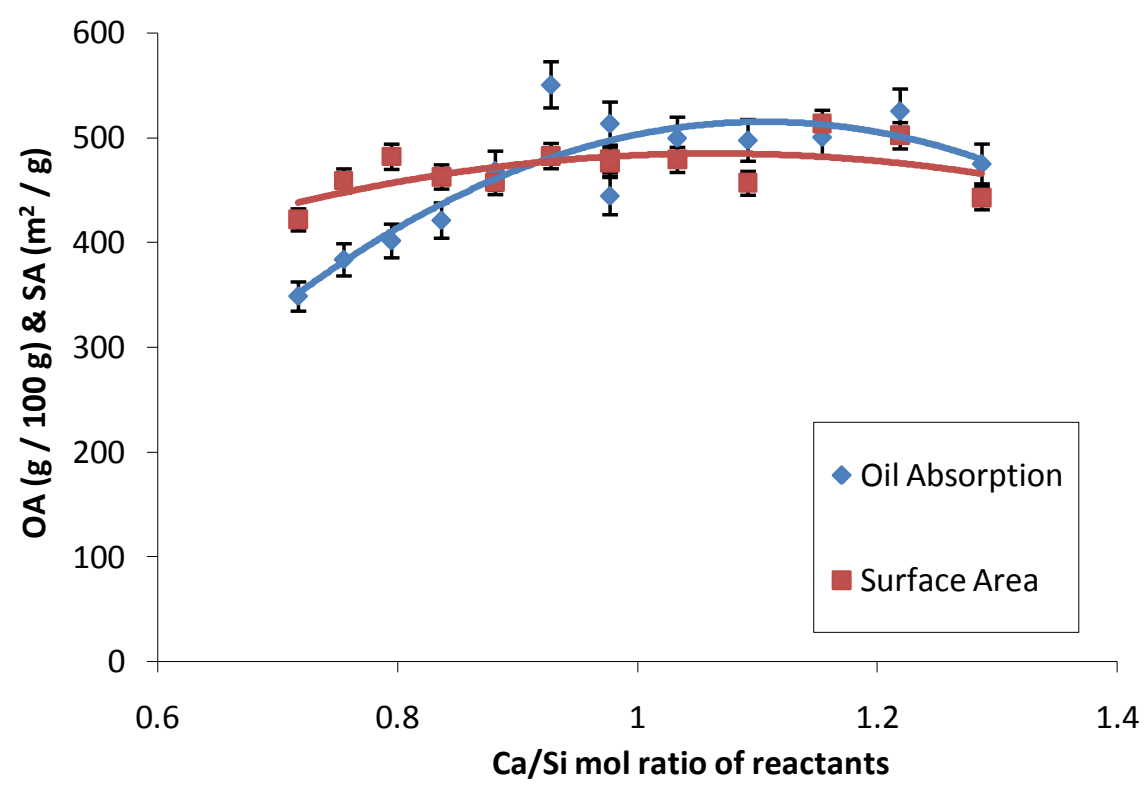

Figure 5.1: Effect of variation in the $\mathrm{Ca} / \mathrm{Si}$ ratio during precipitation upon the physical properties after washing with $2 \mathrm{EE}$.

Samples produced with $\mathrm{Ca} / \mathrm{Si}$ ratios from 1.0 to 1.2 possessed oil absorption capacities within approximately $4 \%$ of this maximum, which is the estimated uncertainty of this test. These results indicate that the $\mathrm{Ca} / \mathrm{Si}$ ratio for the reaction is able to vary by $\pm 10 \%$ and still be able to produce a material with little variation in the total pore volume. This allows a reasonably broad range operating conditions to produce this material. The rogue points whose error bars do not encompass the curves on Figure 5.1 are a result of the general \% uncertainty assigned to the overall method of measurement not truly reflecting the variation that occurs when performing some measurements. This is particularly true for measuring the oil absorption capacity of 2-ethoxyethanol treated samples that have very high capacities $(>400 \mathrm{~g} / 100 \mathrm{~g})$ as the material is more fragile and susceptible to collapse under excessive pressure which can lead to low values being measured. To prevent this collapse, a lighter hand might be used for the test with the result that the linseed oil is not to properly spread throughout the material, which causes the measurement to be higher 
than the true value. The test is always performed multiple times to reduce the likelihood that these difficulties will skew the results and generally produces values that are within the uncertainty range. Unfortunately if multiple flawed measurements are made on the same sample, the final result can fall outside of this range.

It was found that variation of the $\mathrm{Ca} / \mathrm{Si}$ ratio for the reaction had an even smaller effect upon the resulting material's surface area than upon the oil absorption capacity. The maximum surface area was found to occur near a $\mathrm{Ca} / \mathrm{Si}$ ratio of 1.1 , like that of the oil absorption capacity, although there was very little difference across the range examined. The flatter nature of the surface area curve indicates that this property is less affected by the variation in $\mathrm{Ca} / \mathrm{Si}$ ratio than the oil absorption capacity. One possible reason for this could be that the disorder of the atomic structure can allow for more variation in the composition of the platelets while not affecting the macro-structure of the platelet itself. This could result in individual platelets that are formed either more calcium or silicon rich, depending upon which reactant was in excess, but the open-framework of the platelets would still be intact and therefore result in the same bulk physical properties. Based upon the known behaviour of $\mathrm{C}-\mathrm{S}-\mathrm{H}$ (Chen et al., 2004), the excess calcium from reactions that are calcium-rich could be bound in the interlayer cavity rather than water molecules. The structure is likely to be able to accommodate the excess silica by having less calcium in the interlayer spacing and would result in a material that has $\mathrm{Ca} / \mathrm{Si}$ ratio similar to crystalline tobermorite of 0.83 . Materials formed with an even lower $\mathrm{Ca} / \mathrm{Si}$ ratio are probably able to exist due to the disorder of the structure allowing for the absence of some calcium from the Ca-O layers. If the excess of both species can be accommodated within the platelets (up to a limit), no change to either the surface area or the oil absorption capacity would be expected. However, once the disorder that is inherent to the structure could no longer hold the excess species, it is likely that the formation of materials less porous than the calcium silicate, such as precipitated silica or calcium hydroxide would occur (depending upon whether monomeric silica or calcium ions were in excess respectively). This would explain the decrease in both the 
oil absorption capacity and surface area observed as the reactant $\mathrm{Ca} / \mathrm{Si}$ ratio deviates further from 1.

The effect that the differing $\mathrm{Ca} / \mathrm{Si}$ ratio of the reactants had upon the composition of the final product was determined by measuring the monomeric silica and calcium ion concentrations remaining in solution. This would give an indication as to how much excess reactant the material can contain. Depending upon which species was added in excess, the presence of either calcium ions or monomeric silica was measured in solution after the reaction had been completed (Figure 5.2).

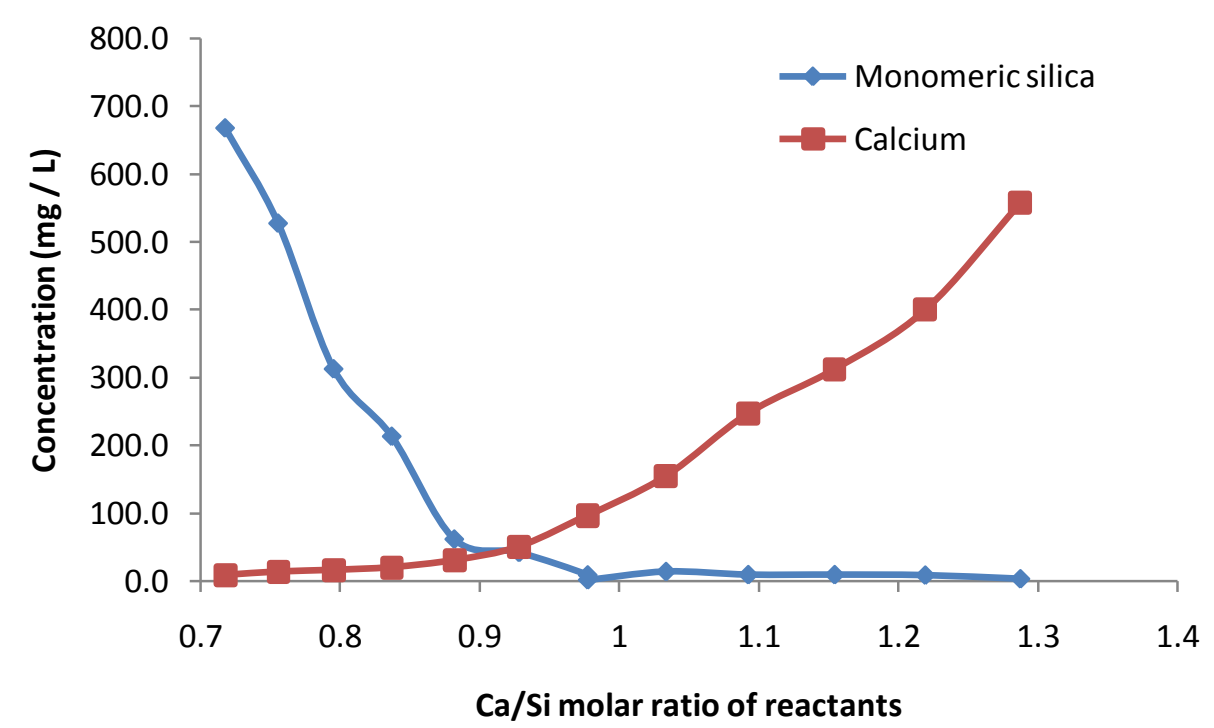

Figure 5.2: The concentration of unreacted species in solution from reactions with varying $\mathrm{Ca} / \mathrm{Si}$ ratios.

However, over the concentration range studied, the excess of each species that remains in solution is less than the increase of the reactant from a $\mathrm{Ca} / \mathrm{Si}$ ratio of 1. This means that while the composition of the final material does not equal the ratio of the reactants, the material does become richer in that component (just to a lesser extent). As an example, when the most calcium-rich material was prepared with a reactant $\mathrm{Ca} / \mathrm{Si}$ ratio of 1.3 , the calcium available for the reaction was increased from 12500 to $16500 \mathrm{mg} / \mathrm{L}$. Of the $4000 \mathrm{mg} / \mathrm{L}$ increase in calcium, only $600 \mathrm{mg} / \mathrm{L}$ was measured to remain in solution unreacted. The concentration of both monomeric silica and calcium ions remaining in solution 
was used to calculate the $\mathrm{Ca} / \mathrm{Si}$ ratio of the nano-structured calcium silicate formed (Table 5.1).

Table 5.1: Calculation of the Ca/Si ratio for nano-structured calcium silicate formed by varying the $\mathrm{Ca} / \mathrm{Si}$ ratio of reactants.

\begin{tabular}{lllclc}
\hline $\begin{array}{l}\text { Reactant } \\
\text { Ca/Si ratio } \\
(\mathrm{mol} / \mathrm{mol})\end{array}$ & $\begin{array}{l}\text { Reactant } \\
\text { silica } \\
\text { concentration } \\
(\mathrm{mg} / \mathrm{L})\end{array}$ & $\begin{array}{l}\text { Calcium } \\
\text { available for } \\
\text { reaction } \\
(\mathrm{mg} / \mathrm{L})\end{array}$ & $\begin{array}{l}\text { Unreacted } \\
\text { silica } \\
\text { concentration } \\
(\mathrm{mg} / \mathrm{L})\end{array}$ & $\begin{array}{l}\text { Unreacted } \\
\text { calcium } \\
\text { concentration } \\
\text { (mg/L) }\end{array}$ & $\begin{array}{l}\text { Calculated } \\
\text { Ca/Si ratio } \\
\text { of product } \\
\text { (mol/mol) }\end{array}$ \\
\hline 1.287 & 19198 & 16480 & 3.8 & 557.5 & 1.244 \\
1.219 & 19198 & 15607 & 9.2 & 400.0 & 1.188 \\
1.154 & 19198 & 14776 & 9.9 & 312.5 & 1.130 \\
1.092 & 19198 & 13984 & 9.9 & 247.5 & 1.073 \\
1.033 & 19198 & 13230 & 14.5 & 155.0 & 1.022 \\
0.977 & 19214 & 12522 & 2.3 & 96.5 & 0.970 \\
0.977 & 19214 & 12522 & 9.5 & 97.5 & 0.970 \\
0.928 & 20232 & 12522 & 42.6 & 51.0 & 0.926 \\
0.882 & 21297 & 12522 & 62.3 & 31.0 & 0.882 \\
0.837 & 22438 & 12522 & 213.6 & 20.5 & 0.843 \\
0.795 & 23613 & 12522 & 312.9 & 16.5 & 0.805 \\
0.755 & 24856 & 12522 & 527.3 & 14.0 & 0.771 \\
0.717 & 26171 & 12522 & 667.6 & 9.0 & 0.736 \\
\hline
\end{tabular}

The relatively small effect that the variation of the reactants $\mathrm{Ca} / \mathrm{Si}$ ratio has upon the oil absorption capacity and surface area, as shown in Figure 5.1, provides flexibility in the possible operating conditions. This flexibility increases the likelihood that the process of producing nano-structured calcium silicate at larger scales will be successful. The flexibility also means that the operating conditions may be selected so that the concentration of species in the waste water may meet any regulatory requirements if necessary, without affecting the quality of the product.

\subsection{Effect of $\mathrm{pH}$ upon precipitation}

Another variable that can affect the composition and structure of material formed is the $\mathrm{pH}$ as this determines the degree of dissociation of the silicic acid and therefore the species that are reacting as well as the solubility of components in the system. As both of the major reactants (calcium hydroxide 
and sodium silicate) are very alkaline, the $\mathrm{pH}$ of the reaction can be controlled by the addition of an acid.

Initial studies into this effect were undertaken before it was known that treatment with 2-ethoxyethanol or the reinforcement procedure could prevent collapse of the structure. Therefore the only method available at that time to analyse a structure that had not collapsed upon drying was by $\mathrm{pH}$ adjusting the calcium silicate slurry that had formed. The use of the more complex $\mathrm{pH}$ adjustment process to prevent structure collapse does introduce more variables than the simple one-step addition of a plug flow of 2-ethoxyethanol but a uniform methodology was used for the treatment of each slurry to minimise these effects. The study involved a series of precipitations using 0.57 mol/L (ca. $34000 \mathrm{mg} / \mathrm{L}$ ) monomeric silica and $0.62 \mathrm{~mol} / \mathrm{L}$ (ca. $25000 \mathrm{mg} / \mathrm{L}$ ) calcium on a $500 \mathrm{~mL}$ scale using the batch method outlined in Chapter 2. Each precipitation took place at a different $\mathrm{pH}$ by altering the initial amount of acid used (Table 5.2) and the $\mathrm{pH}$ of the resulting calcium silicate slurry was subsequently adjusted after the ageing process with samples taken at periodic intervals. The $\mathrm{pH}$ of the reaction was kept above 10 so that the polymerisation of the monomeric silica and subsequent formation of precipitated silica with a network structure would not occur.

Table 5.2: Details of reactions performed to determine the effect of the reaction $\mathrm{pH}$ upon the oil absorption capacity.

\begin{tabular}{cccc}
\hline $\begin{array}{c}\text { Volume of } \mathrm{HCl} \\
\text { added }(\mathrm{mL})\end{array}$ & $\begin{array}{c}\text { Moles of HCl } \\
\text { added per L of } \\
\text { slurry }\end{array}$ & $\begin{array}{c}\text { pH after ageing } \\
\text { for } 1 \mathrm{~h}\end{array}$ & $\begin{array}{c}\text { Maximum oil } \\
\text { absorption achieved } \\
\text { (g/100 g) }\end{array}$ \\
\hline 10.0 & 0.211 & 12.8 & 238 \\
15.0 & 0.326 & 12.5 & 298 \\
20.0 & 0.422 & 12.0 & 258 \\
25.0 & 0.537 & 10.9 & 185 \\
30.0 & 0.633 & 10.6 & 157 \\
35.0 & 0.748 & 10.4 & 142 \\
\hline
\end{tabular}

The optimum $\mathrm{pH}$ for the reaction to give a material with the largest possible total oil absorption capacity was determined by plotting the oil absorption of samples taken during the $\mathrm{pH}$ adjustment for each reaction condition (Figure 
5.3). The precipitation at $\mathrm{pH} 12.5$ produced a material with the largest total oil absorption capacity as the maximum of its curve for the subsequent $\mathrm{pH}$ adjustment with acid was the highest. The other experiments showed that the further the initial reaction $\mathrm{pH}$ was from 12.5 the lower the oil absorption capacity achieved with subsequent $\mathrm{pH}$ adjustment. All three of the reactions prepared at a pH below 11 showed little improvement in oil absorption capacity upon later acidification although the initial samples before being treated with acid all had larger oil absorption capacities. From the initial reaction pHs from 12.8 to 10.9 there was an upward trend in the untreated samples oil absorption capacities.

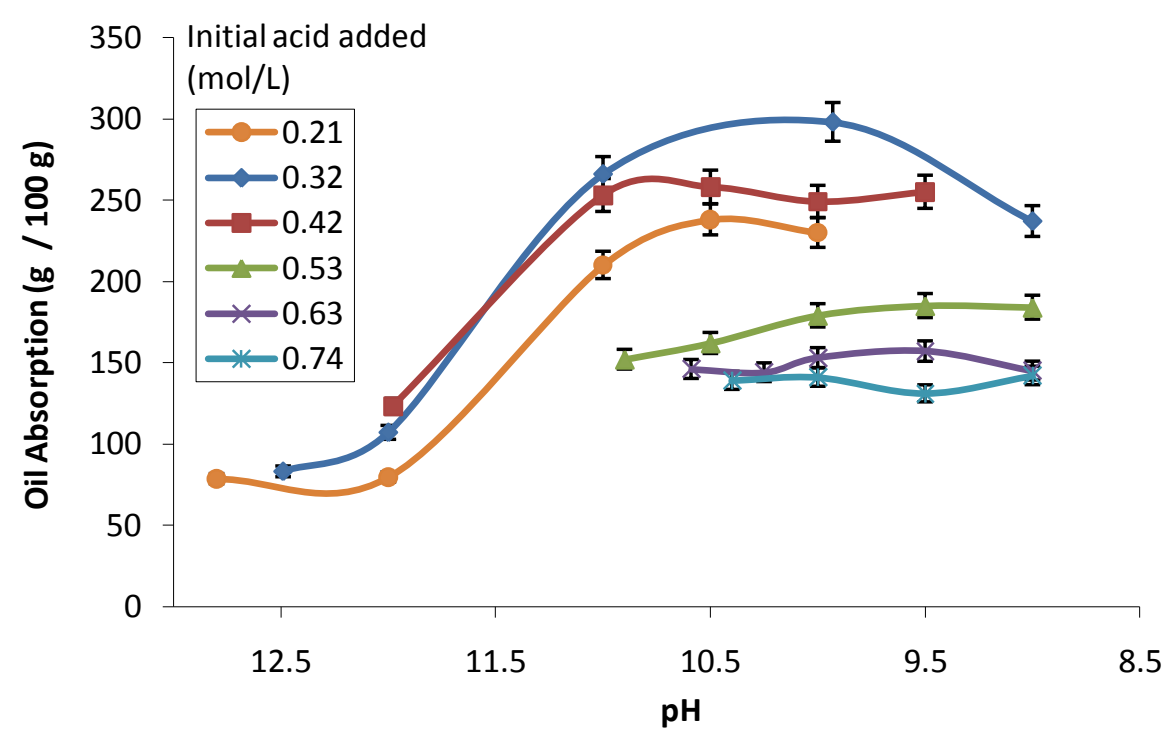

Figure 5.3: Effect of reaction pH upon oil absorption capacity of material formed with $\mathrm{Ca} / \mathrm{Si}$ ratio of 1.1 using $\mathrm{pH}$ adjustment to maintain structure upon drying.

A similar series of $\mathrm{pH}$ adjustments were also performed upon calcium silicates with different $\mathrm{Ca} / \mathrm{Si}$ ratios. The initial materials were prepared by altering the calcium concentration to $0.75 \mathrm{~mol} / \mathrm{L}(30000 \mathrm{mg} / \mathrm{L}$ ) and $0.50 \mathrm{~mol} / \mathrm{L}(20000$ $\mathrm{mg} / \mathrm{L}$ ) while maintaining the same monomeric silica concentration as the initial experiment of $0.57 \mathrm{~mol} / \mathrm{L}(34000 \mathrm{mg} / \mathrm{L})$. Both of these series of experiments also showed that the materials formed around a $\mathrm{pH}$ of approximately 12.5 (although some of the data were fairly well spread) tended to produce materials that upon subsequent lowering of their $\mathrm{pHs}$ formed the largest oil absorption capacity materials (Figure 5.4 and Figure 5.5). 


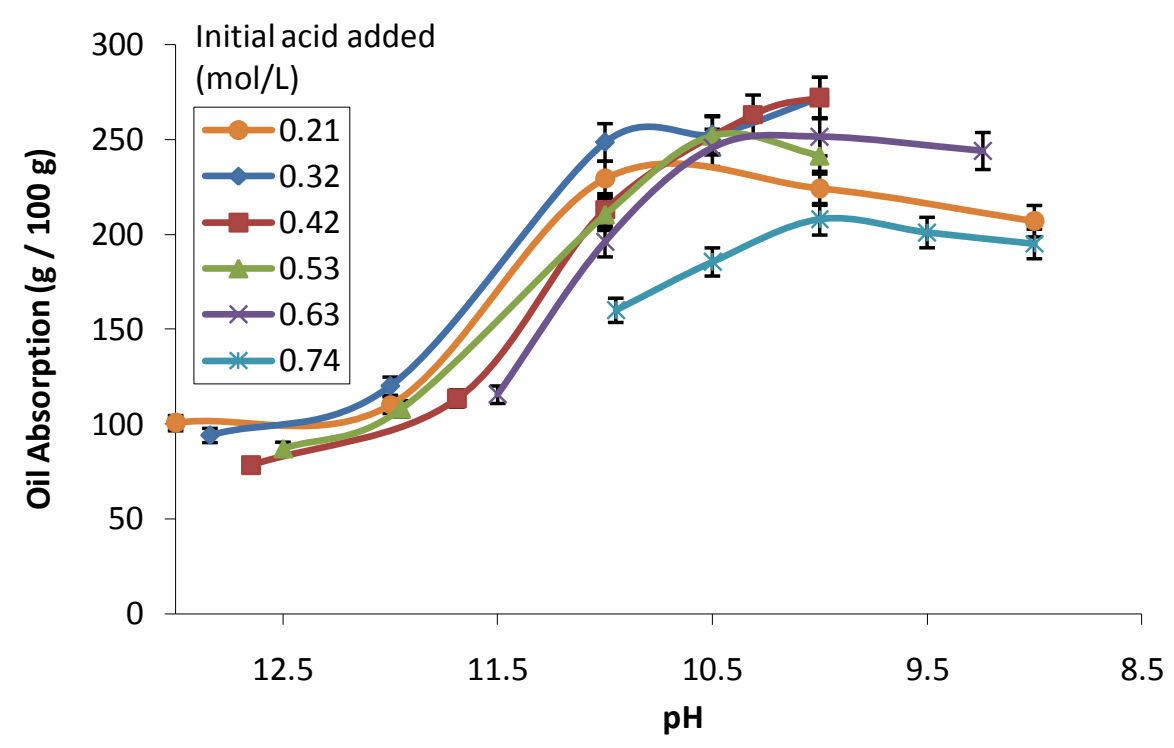

Figure 5.4: Effect of reaction pH upon oil absorption capacity of material formed with $\mathrm{Ca} / \mathrm{Si}$ ratio of 1.3 using $\mathrm{pH}$ adjustment to maintain structure upon drying.

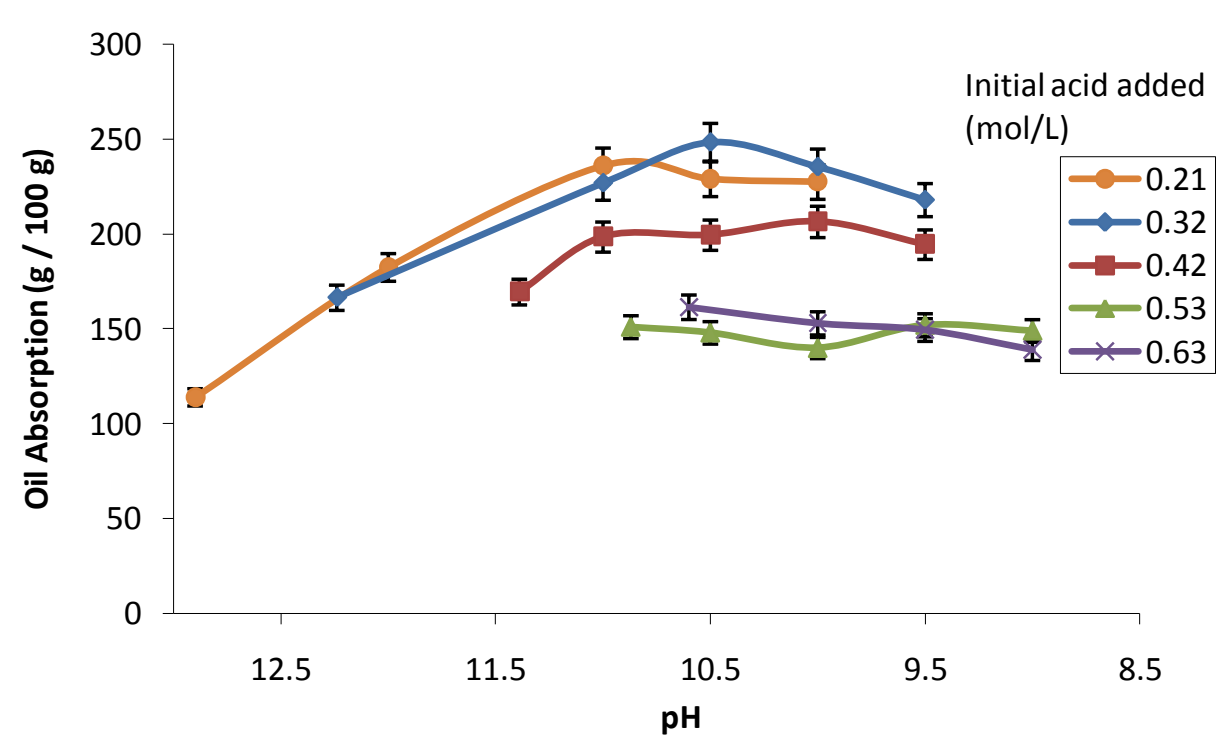

Figure 5.5: Effect of reaction pH upon oil absorption capacity of material formed with $\mathrm{Ca} / \mathrm{Si}$ ratio of 0.9 using $\mathrm{pH}$ adjustment to maintain structure upon drying.

The effect of the $\mathrm{pH}$ for the precipitation of nano-structured calcium silicate upon the resulting materials oil absorption capacity was also studied later in the programme using the simpler method of using 2-ethoxyethanol to maintain the structure following drying. The series of small batch $(150 \mathrm{~mL})$ experiments all used $0.165 \mathrm{~mol} / \mathrm{L}$ monomeric silica and $0.156 \mathrm{~mol} / \mathrm{L}$ of calcium for the precipitation, and all other variables such as stirring times and speeds were kept as uniform as possible (the temperature varied from 21.8 through to $23.4^{\circ} \mathrm{C}$ ). The varying amounts of hydrochloric acid (Table 5.3) added to the 
calcium hydroxide slurry. The reaction of acid and base generated heat, so the slurry was left to stand until it returned to room temperature. The sodium silicate solution was added to a stirred calcium hydroxide slurry and the resulting calcium silicate slurry was stirred for 30 minutes using a magnetic stirrer (so that the mixing was low shear) and then left to stand for six days. The $\mathrm{pH}$ of each slurry was measured 30 minutes into the reaction and again after the six day standing period by which time equilibrium had been reached. After being allowed to settle over the six days, it was noted that some of the slurries had supernatants (Figure 5.6). Each slurry was then mixed using a stirring rod until uniform and then divided approximately into two separate slurries, with one half washed upon filtration with 2-ethoxyethanol to prevent collapse of the structure upon drying and the other washed only with water.

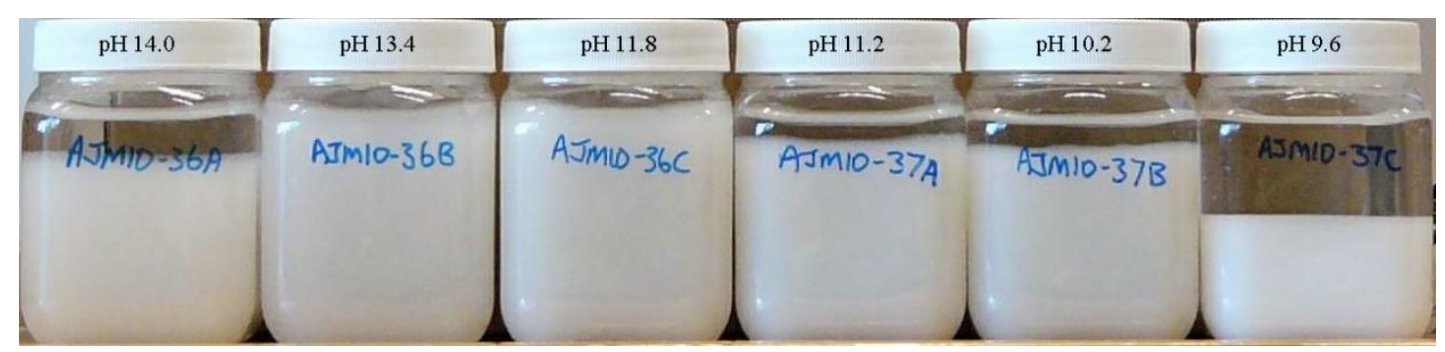

Figure 5.6: A photograph of slurries precipitated at different pHs after being allowed to settle.

From Figure 5.7 it is possible to see that the maximum oil absorption capacity of the 2-ethoxyethanol treated samples occurred at ca. pH 13.0 which is slightly more alkaline than that observed from the somewhat more complicated study using $\mathrm{pH}$ adjustment discussed previously. All of the water washed samples showed signs that the structure had significantly collapsed as the oil absorption capacities were very low. The material produced at $\mathrm{pH} 9.6$ had the highest oil absorption capacity, which is probably a result of forming precipitated silica at this lower pH due to the significant decrease in solubility of silica at ca. pH 10. 
Table 5.3: Details of reactions to determine the effect of pH upon the physical properties of 2-ethoxyethanol treated samples.

\section{Reaction conditions}

Volume of $\mathrm{HCl}$ used (mL)

Concentration of $\mathrm{HCl}$ used

$(\mathrm{mol} / \mathrm{L})$

$\mathrm{pH}$ after $30 \mathrm{~min}$

$\mathrm{pH}$ after 6 days

$\%$ Height of supernatant

\section{Sample details}

Wash treatment

Slurry weight (g)

Filter cake weight $(\mathrm{g})$

Dry weight $\left(110^{\circ} \mathrm{C}\right)(\mathrm{g})$

$\%$ solids in slurry

$\%$ solids in filter cake

Theoretical total weight

(g) a

Average oil absorption

(g/100 g)

Surface area $\left(\mathrm{m}^{2} / \mathrm{g}\right)$

\begin{tabular}{c|c}
\hline 0.00 & 1.00 \\
\hline 0.00 & 0.07 \\
\hline 13.7 & 13.2 \\
14.0 & 13.4 \\
21 & 0
\end{tabular}

2EE water 2EE water 2

74.273 .6

$74.2 \quad 73.6$

$74.4 \quad 72.0$

$\begin{array}{ll}74.4 & 72.0 \\ 29.0 & 33.2\end{array}$

$18.7 \quad 18.3$

$1.73 \quad 1.69$

$2.3 \quad 2.3$

$\begin{array}{ll}2.3 & 2.3 \\ 9.3 & 9.2\end{array}$

1.651 .58

$2.2 \quad 2.2$

5.7

$\begin{array}{ll}5.7 & 4.8\end{array}$

$\begin{array}{cc}2 \mathrm{EE} & \text { water } \\ 73.1 & 75.2\end{array}$

$27.4 \quad 31.3$

$27.4 \quad 31.3$

$1.52 \quad 1.53$

$2.1 \quad 2.0$

$5.5 \quad 4.9$

3.02

3.21

\begin{tabular}{l|l}
496 & 75 \\
480 & 52
\end{tabular}

\begin{tabular}{|c|c|c|c|c|c|c|c|c|c|}
\hline 643 & 34 & 443 & 48 & 201 & 115 & 206 & 134 & 228 & 155 \\
\hline 453 & 37 & 378 & 122 & 101 & 93 & 106 & 98 & 66 & 66 \\
\hline
\end{tabular}

\footnotetext{
a Calculated on the basis that all material was water washed and dried at $110^{\circ} \mathrm{C}$.
} 


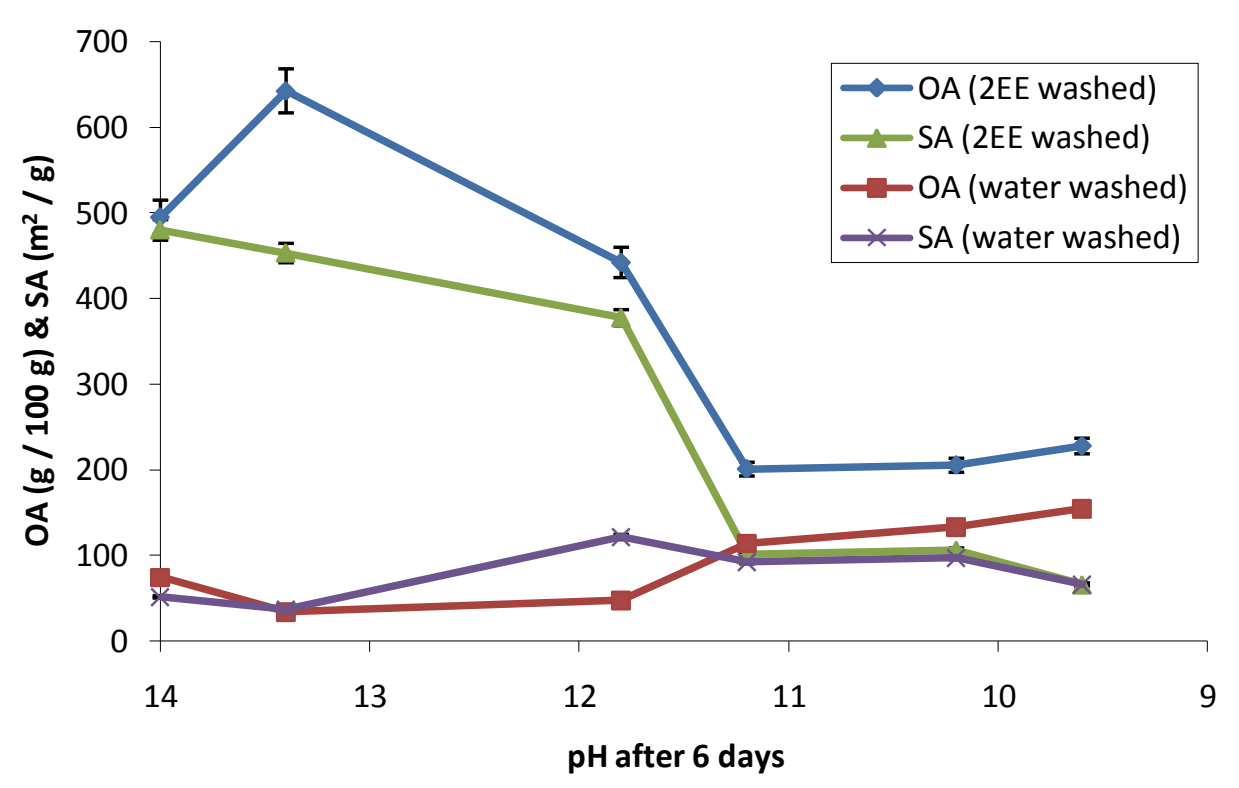

Figure 5.7: Effect of reaction pH upon the oil absorption capacity and surface area of 2ethoxyethanol treated samples.

As the slurries had been split in two and each half treated differently for characterisation, the total yield of each reaction was calculated by multiplying the sum of the split slurry weights by the percent solids of the water washed slurry. This eliminates any discrepancy caused by the slurries not being split by exactly the same proportion in each case as the 2-ethoxyethanol washed samples typically weigh more per unit volume when dried at $110^{\circ} \mathrm{C}$, as has been previously discussed in section 4.1. It can be seen in Figure 5.8, that the $\mathrm{pH}$ of the reaction also has an effect upon the mass of material produced when dried at $110^{\circ} \mathrm{C}$. This is likely to be due in part to the trend of the surface area of the material increasing as the $\mathrm{pH}$ of the reaction increases as this allows for more groups to be bound to the surface, with the material produced at the highest $\mathrm{pH}$ possessing a surface area over seven times that of the material produced at the lowest $\mathrm{pH}$. This relationship between the $\mathrm{pH}$ used to produce the material and the resulting surface area of the material is of significance for the preparation of a material that would be used in applications such as for ion-exchange where a large accessible surface area is an important property. While the oil absorption capacity for this material is not the largest in this series of preparations, it is still very significant compared with other materials and would aid the diffusion of species through the material. 


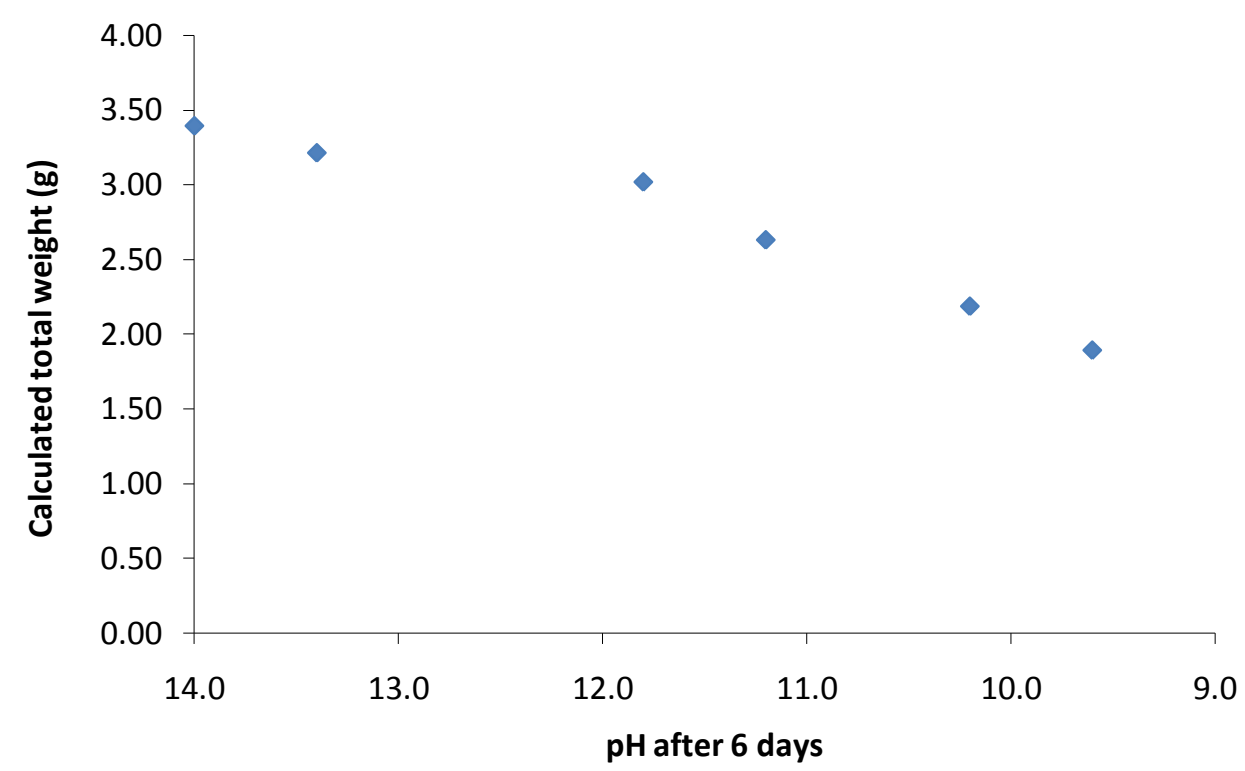

Figure 5.8: Effect of $\mathrm{pH}$ upon the calculated total yield of precipitate formed.

\subsection{Effect of changing both calcium and hydroxide concentrations}

The use of calcium hydroxide as the calcium source for the precipitation of nano-structured calcium silicate was chosen as the ideal $\mathrm{pH}$ for the reaction to produce a material with a large oil absorption capacity and high surface area is very alkaline. The result of using this calcium source is therefore two-fold in that it both provides calcium ions for the precipitation and hydroxide ions for increasing the $\mathrm{pH}$. The result of both species coming from the same source is that the effect of each individually cannot be studied without the addition of an acid. Therefore a series of experiments was carried out whereby calcium chloride was used as the source of calcium ions for the precipitation, and sodium hydroxide was used as a means of providing an independent source of hydroxide ions to increase the alkalinity. Based upon the result the produced the maximum oil absorption capacity from using calcium hydroxide, the ideal case was calculated so that the reaction using calcium chloride and sodium hydroxide would duplicate these conditions. The concentration of hydroxide ions necessary were calculated by subtracting the number of moles of protons usually provided by the hydrochloric acid from the number of moles of 
hydroxide ions usually provided by the calcium hydroxide. Reaction conditions to duplicate the ideal case were $0.57 \mathrm{~mol} / \mathrm{L}$ monomeric silica (which was kept constant for all reactions) and $0.62 \mathrm{~mol} / \mathrm{L}$ of calcium and $0.94 \mathrm{~mol} / \mathrm{L}$ of hydroxide ions. Both the calcium and hydroxide ion concentrations were then varied by decreasing each species to $3 / 4,1 / 2$, and $1 / 4$ of their ideal concentration to form a grid of sixteen reaction conditions (Table 5.4). After reacting the calcium and hydroxide ion slurry with monomeric silica, the pHs of the resulting slurries increase with the increasing hydroxide ion concentration and the decreasing calcium concentration.

Table 5.4: The pH of each slurry produced following reaction with monomeric silica by varying the hydroxide and calcium ion concentrations.

\begin{tabular}{lc|cccc} 
& & 0.94 & 0.70 & 0.47 & 0.23 \\
\cline { 2 - 5 } Calcium & 0.62 & 11.7 & 11.1 & 10.7 & 9.8 \\
concentration & 0.47 & 13.2 & 12.4 & 11.0 & 10.0 \\
available for & 0.31 & 13.5 & 13.3 & 12.6 & 10.5 \\
reaction $^{1}$ & 0.16 & 13.7 & 13.5 & 13.3 & 12.2 \\
(mol/L) & & & &
\end{tabular}

${ }^{1}$ Due to the low solubility of $\mathrm{Ca}(\mathrm{OH})_{2}$ of $0.12 \mathrm{~g} / 100 \mathrm{~g}$ of water at $25{ }^{\circ} \mathrm{C}$ (Aylward \& Findlay, 2002), these are not the concentrations of calcium ions in the reactant slurry. However, the reaction with monomeric silica removes available calcium ions from the solution and consequently causes the progressive dissolution of all of the calcium hydroxide, thereby allowing all of the calcium to be available for the reaction.

The maximum oil absorption achieved for a 2-ethoxyethanol treated sample through this series of experiments occurs at what had been previously determined to be the ideal concentrations (Figure 5.9). The $\mathrm{pH}$ for the resulting slurry is slightly lower than anticipated, possibly due to the larger uncertainty in measuring the $\mathrm{pH}$ above the highest calibration point of $\mathrm{pH} 10$. A similarly high oil absorption capacity was measured for the sample produced with the ideal calcium concentration ( $0.62 \mathrm{~mol} / \mathrm{L})$ but at the less alkaline $\mathrm{pH}$ of 11.1 . The sample produced with $3 / 4$ of the calcium concentration $(0.47 \mathrm{~mol} / \mathrm{L})$ and the full hydroxide concentration (0.94 mol/L) had an oil absorption capacity approximately within the uncertainty of the measured for reaction based upon the ideal conditions. Overall it was found that large variations away from what 
had previously been determined as the optimised conditions resulted in materials with greatly decreased oil absorption capacities while slight variations did not have a large effect.

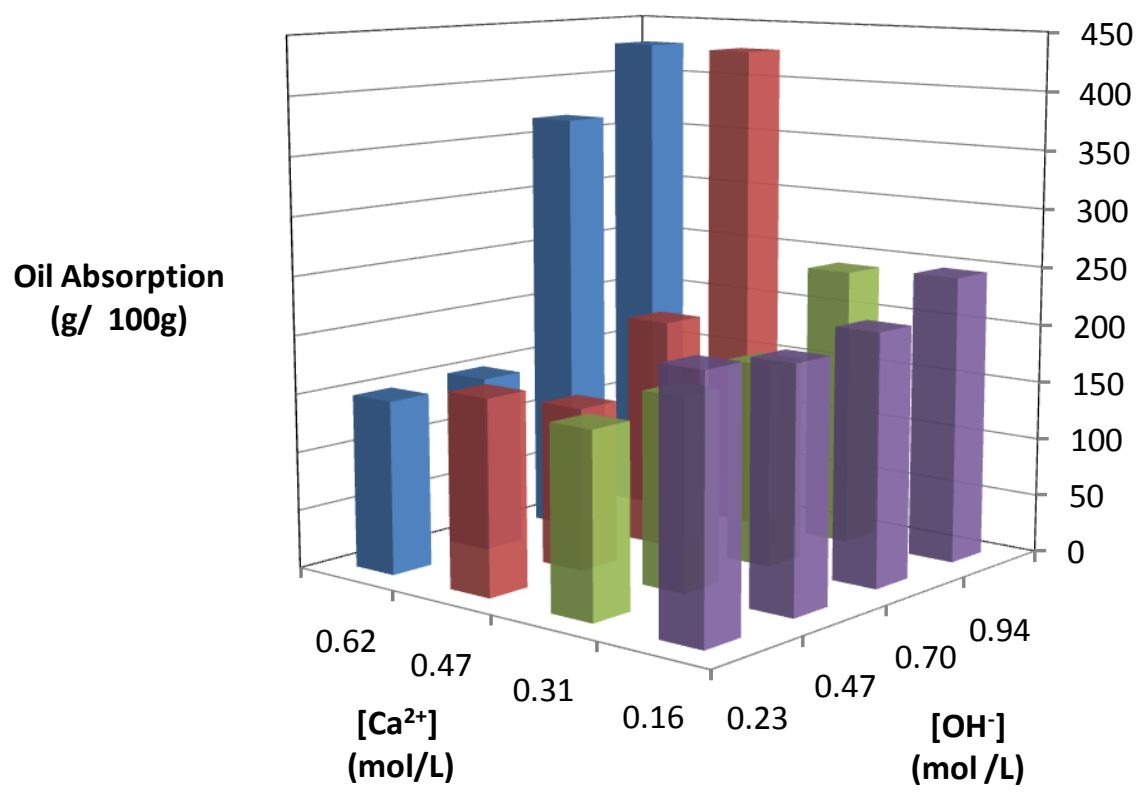

Figure 5.9: The oil absorption capacity of 2-ethoxyethanol treated samples produced with varying hydroxide and calcium concentrations.

The slightly higher oil absorption capacity of the samples precipitated with the minimum calcium concentration does indicate the importance of the $\mathrm{pH}$ as all of the slurries produced under these conditions had higher $\mathrm{pHs}$ that are closer to the optimum range. The oil absorption capacity of samples that had not been treated with 2-ethoxyethanol are a reflection of the degree of collapse that the structure has suffered (Figure 5.10). The highest values were obtained for those samples produced at the lowest pHs possibly due to the increased density of hydroxyl groups on the surface of samples in a higher $\mathrm{pH}$ environment that therefore increases the forces upon the structure during water removal. For comparison, a dry weight of each total slurry was calculated on the basis that the whole sample was washed only with water and dried at $110{ }^{\circ} \mathrm{C}$ as each of the slurries were divided approximately in half to be treated separately. As expected from a decrease in one of the reactants, the weight of product decreases as the proportion of calcium decreases with the material formed using the previously determined ideal conditions producing the greatest amount of product (Figure 5.11). 


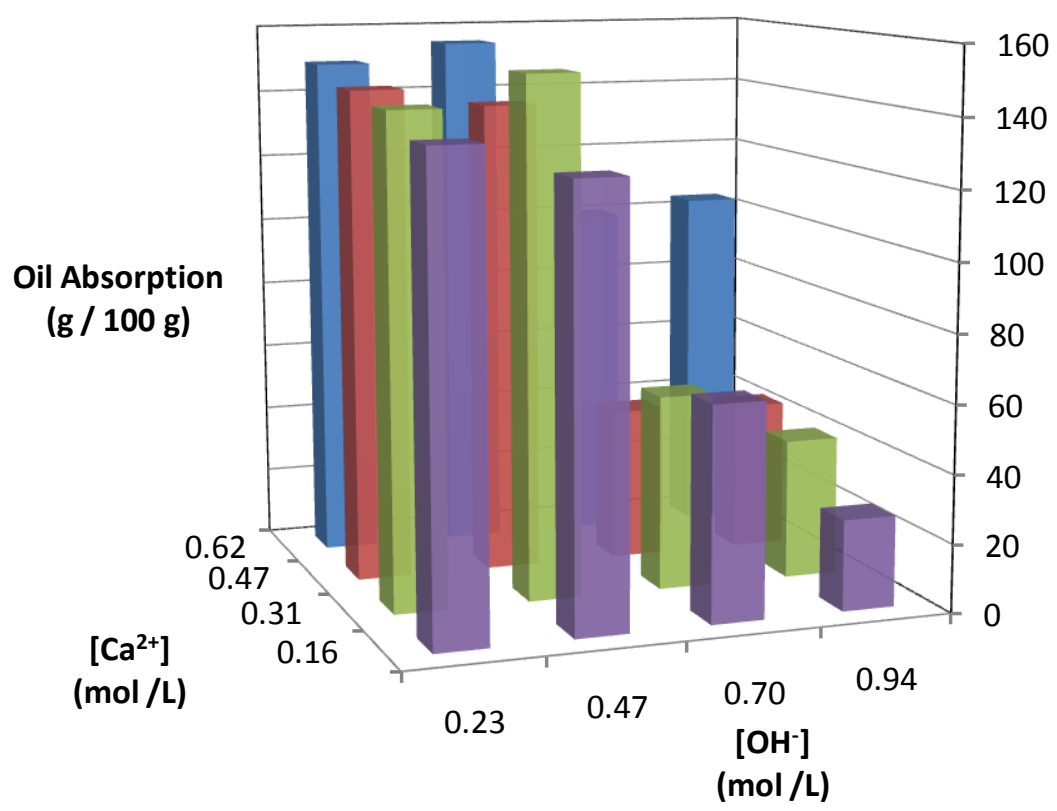

Figure 5.10: The oil absorption capacity of untreated samples produced with varying hydroxide and calcium concentrations.

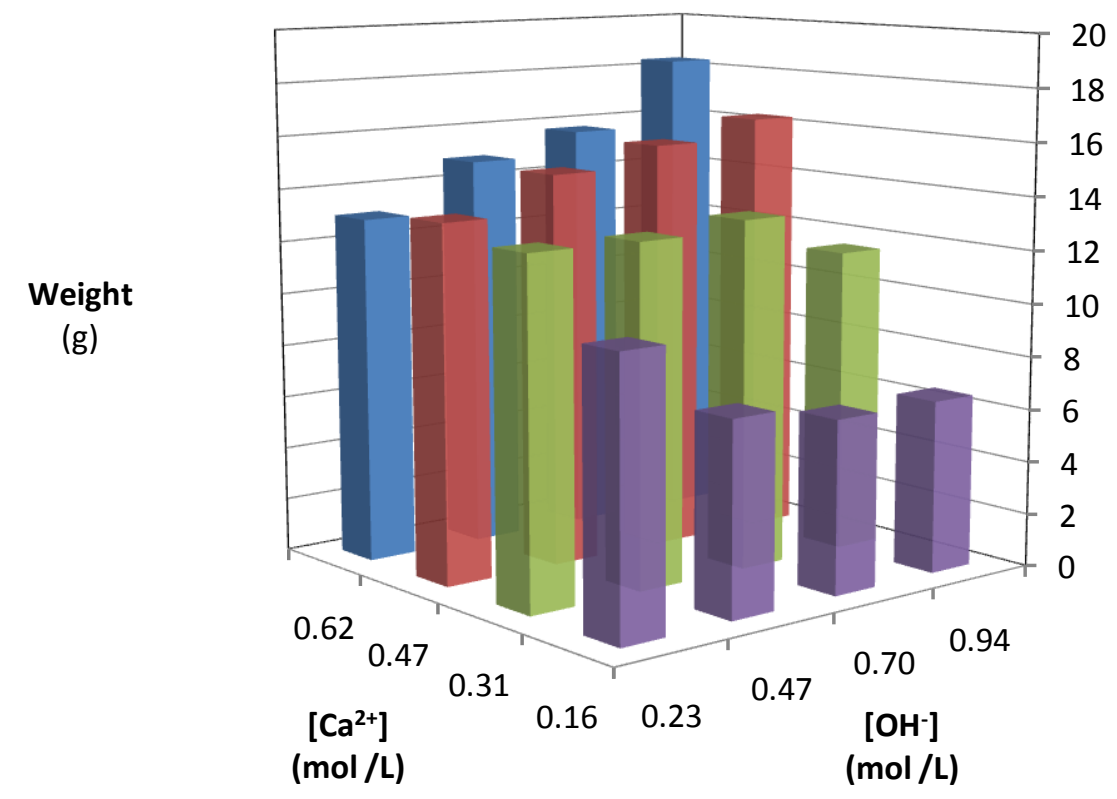

Figure 5.11: The calculated total weight of material produced with varying hydroxide and calcium concentrations. 


\subsection{Effect of temperature upon precipitation}

One of the variables studied for the formation of nano-structured calcium silicate was the temperature of the reaction. It was thought that by increasing the temperature, the reaction rate could be increased and therefore reduce the amount of time required for the re-organisation that occurs during the ageing process. A reduction in the reaction time is of importance for the scaling-up of the process for commercialisation as this determines the size of tanks necessary to have the residence time required to keep the material agitated throughout this process. The reaction was performed after letting the reactants equilibrate at the desired temperature before mixing, with only initial stirring to ensure effective mixing at the time of reaction. The ageing process was allowed to proceed without any agitation to reduce the number of variables that could effect the materials development. Several samples were taken during the ageing process after brief gentle stirring by hand to ensure the sample was representative. These samples were treated with 2-ethoxyethanol to prevent structure collapse. As can be seen in Figure 5.12, the result of performing the reaction at an elevated temperature of $60^{\circ} \mathrm{C}$ was not only no decrease in the ageing time until a product with constant pore volume was obtained, but also that the final total pore volume was lower than the material reacted at room temperature. It is also possible that as the solubility of calcium hydroxide decreases with increasing temperature, that this may have interfered with the initial formation of the material by changing the relative concentrations at the point of precipitation.

Another reaction was performed using the same methodology but at $2^{\circ} \mathrm{C}$ to determine if an increase in available calcium at the point of precipitation may be beneficial. If the reaction rate was slowed at the lower temperature, it was thought that by prolonging the self-organising process during ageing could result in a more highly ordered material with a larger pore volume. The low temperature reaction resulted in an oil absorption of $550 \mathrm{~g} / 100 \mathrm{~g}$ after the reaction mixture had been left to age for 3 days, which is similar to that achieved at room temperature over several hours. 


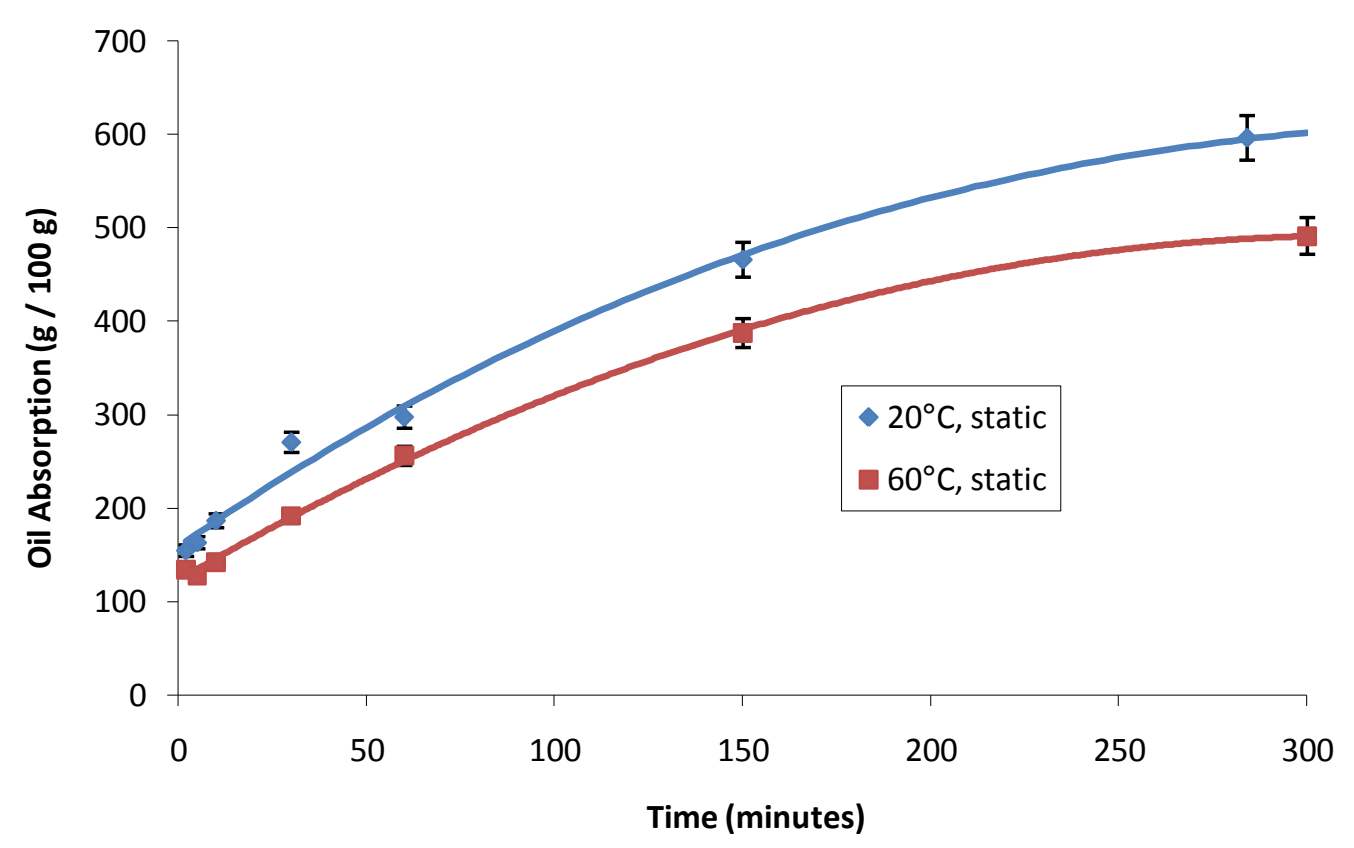

Figure 5.12: Effect of reaction temperature upon the development of oil absorption capacity of 2-ethoxyethanol treated material.

\subsection{Effect of scale and mixing}

One of the greatest challenges in the scaling up of a chemical process is to reproduce the effectiveness of mixing from the small scale used in the laboratory to the large scale required for industrial applications. With this in mind it is important to understand the role that the mixing has in any particular process. Two reactions were performed where the only difference was the use of stirring during the ageing process. In one case the stirring was used throughout the ageing process, whereas for the other the stirring was only used for the first minute after mixing the two reactants. Each reaction was carried out on a $1 \mathrm{~L}$ scale using $0.165 \mathrm{~mol} / \mathrm{L}$ monomeric silica, $0.156 \mathrm{~mol} / \mathrm{L}$ calcium, and $0.122 \mathrm{~mol} / \mathrm{L}$ hydrochloric acid using the batch method as detailed in section 2.2.1. The development of the structure was monitored by measuring the oil absorption capacity of samples collected during the ageing process after treating them with 2-ethoxyethanol to prevent collapse of the structure upon drying. It was found that both of the reactions with and without stirring reached approximately the same maximum oil absorption and although the reaction with stirring did reach a plateau earlier. High speed mixing throughout 
the whole process was not necessary to produce material with a high oil absorption capacity (Figure 5.13). This result influenced the design of the continuous process that has a small initial reaction vessel that is vigorously stirred to allow effective mixing and therefore ensure homogeneity for the initial precipitation followed by a large ageing vessel that is gently stirred. As the high shear mixing of a large volume of slurry is not necessary, the equipment necessary to operate the pilot plant is considerably simpler.

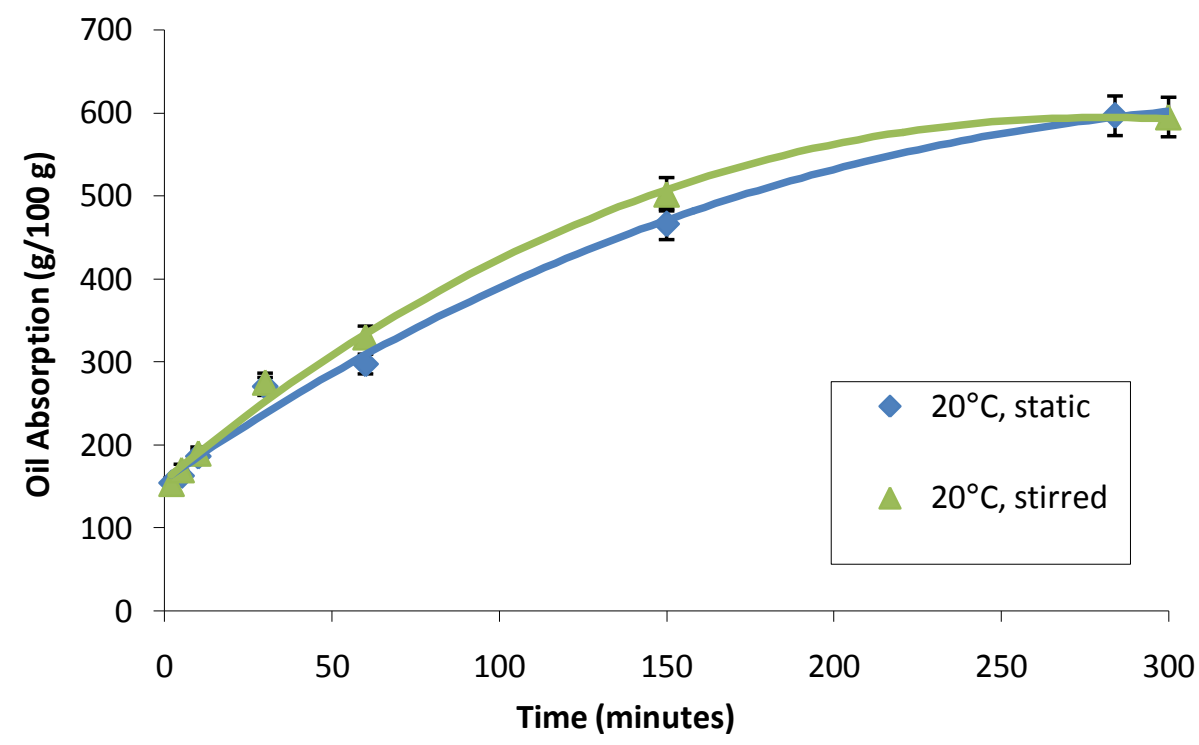

Figure 5.13: The effect of stirring throughout the ageing process upon the development of oil absorption capacity of 2-ethoxyethanol treated material.

\subsection{The effect of concentration}

While the concentration of the reaction is not of particular importance at a laboratory scale in order to produce sufficient quantities for analysis, the same cannot be said for production on a commercial scale. With the cost of reactors effectively increasing exponentially with size, the ability to produce the maximum amount of product with the desired properties per reactor volume is of great importance. A series of experiments was performed whereby the concentration of the reactants was altered proportionately while all other variables were kept constant and the products characterised after being allowed to age for 1 day to ensure development of the structure was complete. The samples were all treated with 2-ethoxyethanol to prevent structure collapse upon drying. Figure 5.14 shows that up to a monomeric silica 
concentration of ca. $0.4 \mathrm{~mol} / \mathrm{L}$ there is no real difference in the total pore volume of the structure, but at higher concentrations, the oil absorption capacity begins to drop away.

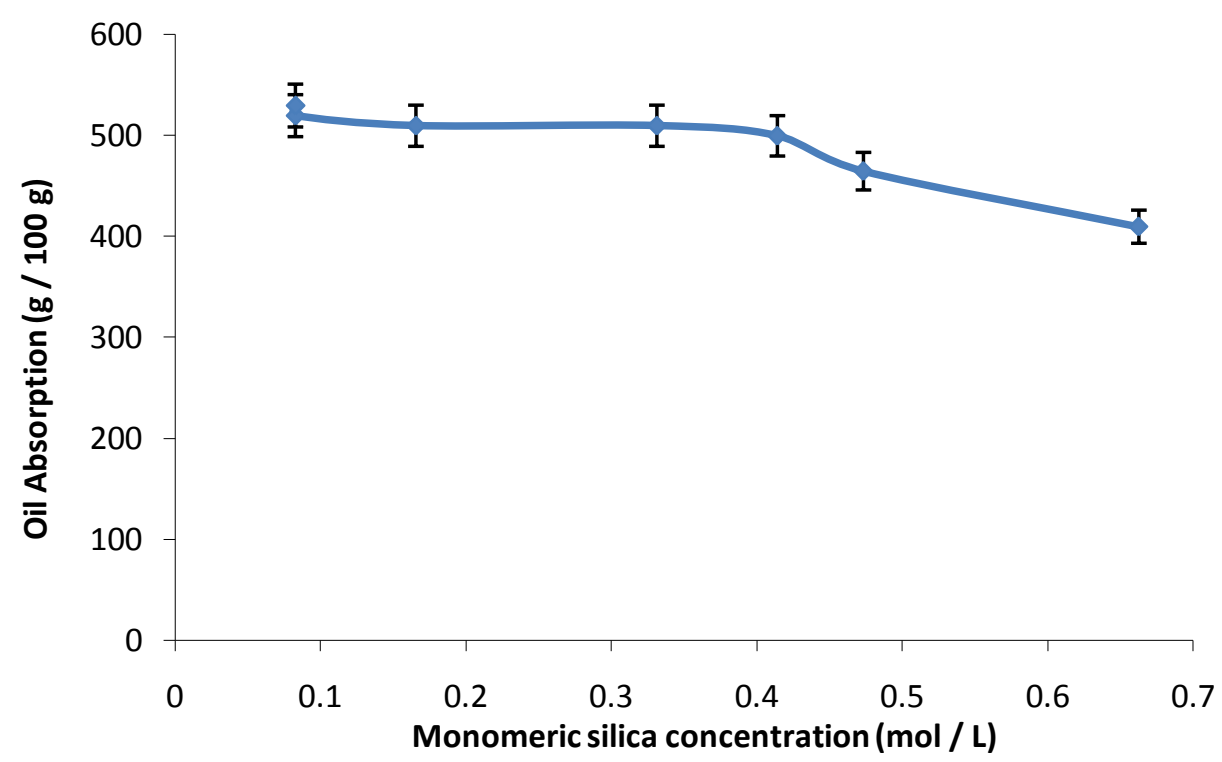

Figure 5.14: The effect of concentration upon the maximum oil absorption achievable with a 2ethoxyethanol treated material.

There is a noticeable difference in the viscosity of the slurry around this concentration, with slurries of ca. $0.4 \mathrm{~mol} / \mathrm{L}$ or less being free flowing and easily stirred and pumped. Above ca. $0.4 \mathrm{~mol} / \mathrm{L}$ the slurry is more viscous and requires more power to stir which can lead to two possible causes to this reduction in oil absorption capacity. One likely cause is that despite increasing the speed of the stirrer to keep the slurry flowing, the mixing at the point of precipitation may not be effective enough to create a homogenous slurry that may result in local areas of different composition reacting. The other possible cause of reducing the oil absorption capacity may be that increasing the speed of the stirrer to improve mixing, has created sufficient shear at the edge of the propeller blades to damage the open structure that is formed. While it is practically possible to perform the reaction with a monomeric silica concentration up to ca. $0.7 \mathrm{~mol} / \mathrm{L}$, the reduction in maximum achievable oil absorption capacity at this concentration resulted in selecting a monomeric silica concentration of $0.4 \mathrm{~mol} / \mathrm{L}$ to be commonly used for most experiments. It is possible that the use of a different mixing system may be able to enable more 
effective stirring without any adverse effects to the structure and therefore allow a greater amount of material per reactor volume to be produced.

\subsection{Summary}

The large pore volume resulting from the way the platelets stack to form an open framework structure distinguishes nano-structured calcium silicate from many other materials. Therefore most of this study has focussed on improving this property. Through the optimisation of the reaction conditions and the development of several different post-formation methods to maintain the structure, nano-structured calcium silicates with maximised pore volumes or surface areas have been developed that are suitable for various applications. The typical properties of the modified nano-structured calcium silicates prepared under optimum conditions are given in Table 5.5. The first steps for producing all of the materials are universal with modifications of the material coming about as a result of different methods of treating the same base slurry. A general schematic for the production of the most commonly produced materials is given in Figure 5.15.

Table 5.5: Typical properties of differently treated nano-structured calcium silicates prepared using conditions for optimising the oil absorption capacity.

\begin{tabular}{lccc}
\hline Material & $\begin{array}{c}\text { Oil absorption } \\
(\mathrm{g} / 100 \mathrm{~g})\end{array}$ & $\begin{array}{c}\text { Surface area } \\
\left(\mathrm{m}^{2} / \mathrm{g}\right)\end{array}$ & $\begin{array}{c}\text { Ca/ Si ratio } \\
(\mathrm{mol} / \mathrm{mol})\end{array}$ \\
\hline $\begin{array}{l}\text { Water washed } \\
\text { (untreated) }\end{array}$ & 120 & 140 & 1.00 \\
$\begin{array}{l}\text { 2-Ethoxyethanol } \\
\text { washed }\end{array}$ & $500-600$ & $500-550$ & 1.00 \\
Reinforced & 400 & 300 & 0.56 \\
Neutral pH & 350 & 350 & 0.10 \\
\hline
\end{tabular}

${ }^{1}$ Determined with X-ray fluorescence.

A mass balance for an optimised process on a $10 \mathrm{~L}$ scale using a monomeric silica concentration of $0.43 \mathrm{~mol} / \mathrm{L}$, a calcium concentration of $0.47 \mathrm{~mol} / \mathrm{L}$, and a hydrochloric acid concentration of $0.25 \mathrm{~mol} / \mathrm{L}$ is given in Table 5.6. The base slurry contains $5.9 \%$ solids $(\mathrm{w} / \mathrm{w})$ while the slurry following reinforcement contains $4.8 \%$ solids $(\mathrm{w} / \mathrm{w})$. 


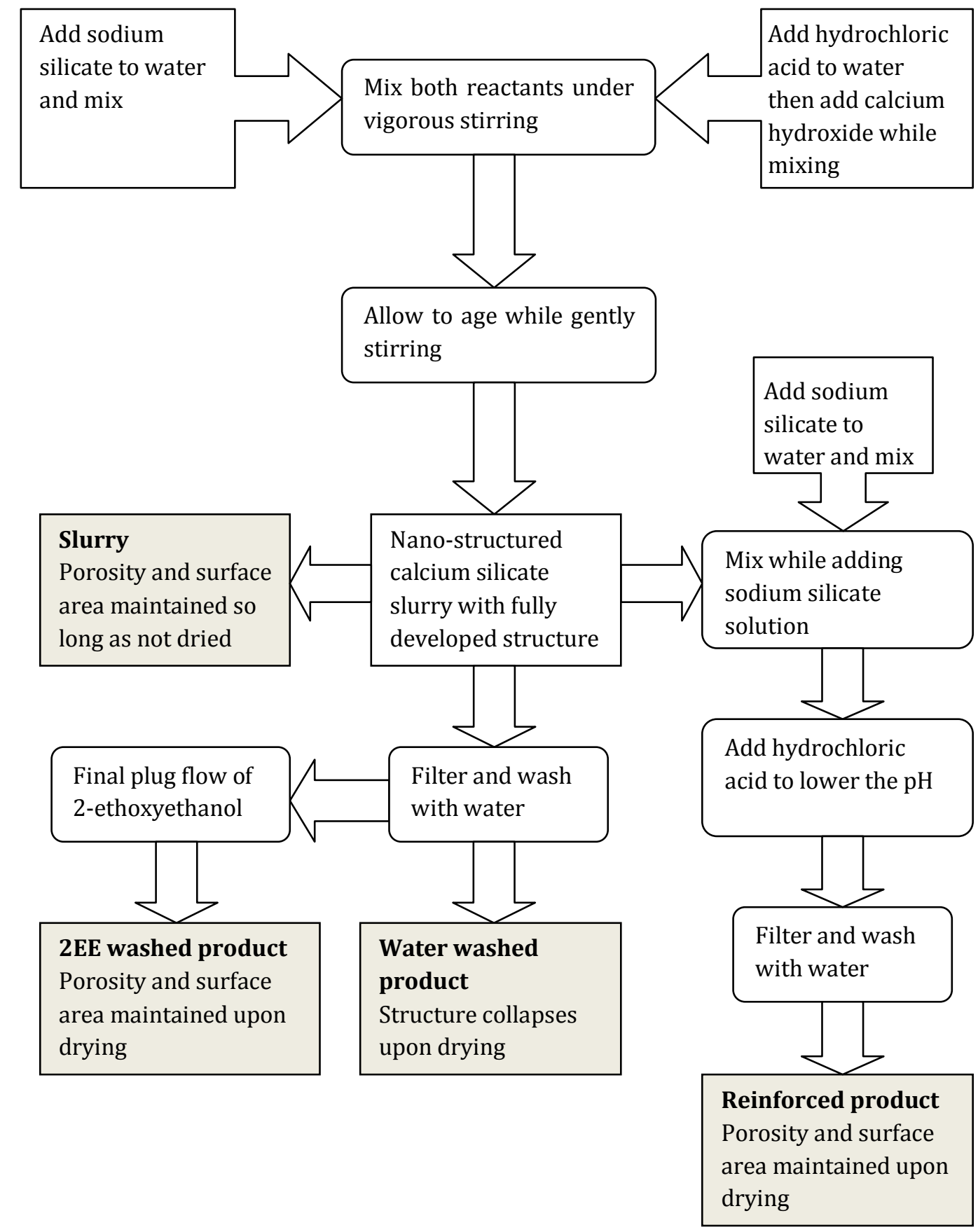

Figure 5.15: Schematic showing the general steps for the production of different types of nano-structured calcium silicates.

The concentrations of the major species that remain in the solution of the base nano-structured calcium silicate are given in Table 5.7. The concentrations of both sodium ions and chloride ions in the filtrate have been estimated on the assumption that approximately all of these species from the sodium silicate and the hydrochloric acid respectively remain in solution and are not included in the solid product. The concentration of species in the waste water from the 
reaction will in reality be more dilute due to the addition of water from washing the material and the figures given should be treated as maximum concentrations.

Table 5.6: A mass balance for the optimised preparation of nano-structured calcium silicate in untreated and modified forms.

\begin{tabular}{llcc}
\hline Formation of the base slurry & Weight & Volume \\
& & $(\mathrm{g})$ & $(\mathrm{ml})$ \\
\hline $\mathrm{A}$ & Sodium silicate (26.3\% Si02) & 980.3 & 705.2 \\
& Water & 4295 & 4295 \\
& Total & 5275 & 5000 \\
& & & \\
& Calcium hydroxide & 347.3 & 149.7 \\
& Hydrochloric acid (33\%) & 279.4 & 240.0 \\
& Water & 4610 & 4610 \\
& Total & 5237 & 5000 \\
& Slurry from A+B & 10510 & 10000
\end{tabular}

This slurry can be used the following ways:

\#1: No further treatment - just filtration and plug flow washing with water

Dried Product $\left(110^{\circ} \mathrm{C}\right)$

621.7

\#2: Final plug flow wash of 2-ethoxyethanol

Dried Product $\left(110^{\circ} \mathrm{C}\right)$

657.5

\section{\#3: Reinforcement}

\begin{tabular}{llrr} 
C & Slurry & 10510 & 10000 \\
D & Sodium silicate $(26.3 \%$ SiO2) & 700.0 & 503.6 \\
& Water & 4496 & 4496 \\
& Total & 5196 & 5000 \\
& C $+\mathrm{D}$ & 15710 & 15000 \\
E & $33 \% \mathrm{HCl}$ & 220.8 & 189.7 \\
& & & \\
& E+F & 15930 & 15190 \\
& Dried Product $\left(110^{\circ} \mathrm{C}\right)$ & 760.0 & \\
\hline
\end{tabular}


Table 5.7: Concentration of major species in solution from the preparation of the base nanostructured calcium silicate slurry.

\begin{tabular}{lcc}
\multicolumn{3}{l}{ Waste solution (filtrate) } \\
$\mathrm{pH}$ & 11.9 & \\
& & \\
\hline & Weight & Conc. \\
Main species present: & $(\mathrm{g})$ & $(\mathrm{mg} / \mathrm{L})$ \\
\hline $\mathrm{Na}^{+}$ & 66.0 & 6603 \\
$\mathrm{Cl}^{-}$ & 55.9 & 5594 \\
$\mathrm{Ca}^{2+}$ & 4.5 & 445 \\
$\left(\mathrm{SiO}_{2}\right)_{\mathrm{m}}$ & 2.0 & 200 \\
\hline
\end{tabular}

This optimised method has been successfully employed at a pilot plant scale to repeatedly produce $250 \mathrm{~L}$ of reinforced nano-structured calcium silicate by using the continuous process. Photographs of the pilot plant are shown in the foreground of Figure 5.16, Figure 5.17, and Figure 5.18. The chemical feed tanks were constantly mixed with individual overhead stirrers and had taps at the base to which tubing was connected to a dual headed peristaltic pump. The pump drew both feed stocks at the same rate and expelled the solutions into a small reaction vessel that was vigorously mixed with an overhead stirrer. The product then overflowed from this vessel into the large ageing tank that was gently mixed by another overhead stirrer for the structure to develop. Following the development of the structure, the reinforcement process was undertaken by pumping the additional diluted sodium silicate from the same tank used previously for storing the sodium silicate solution directly into the ageing tank by using the peristaltic pump. The diluted acid required to decrease the $\mathrm{pH}$ of the slurry to 10 was then steadily added by hand using a $20 \mathrm{~L}$ bucket. 


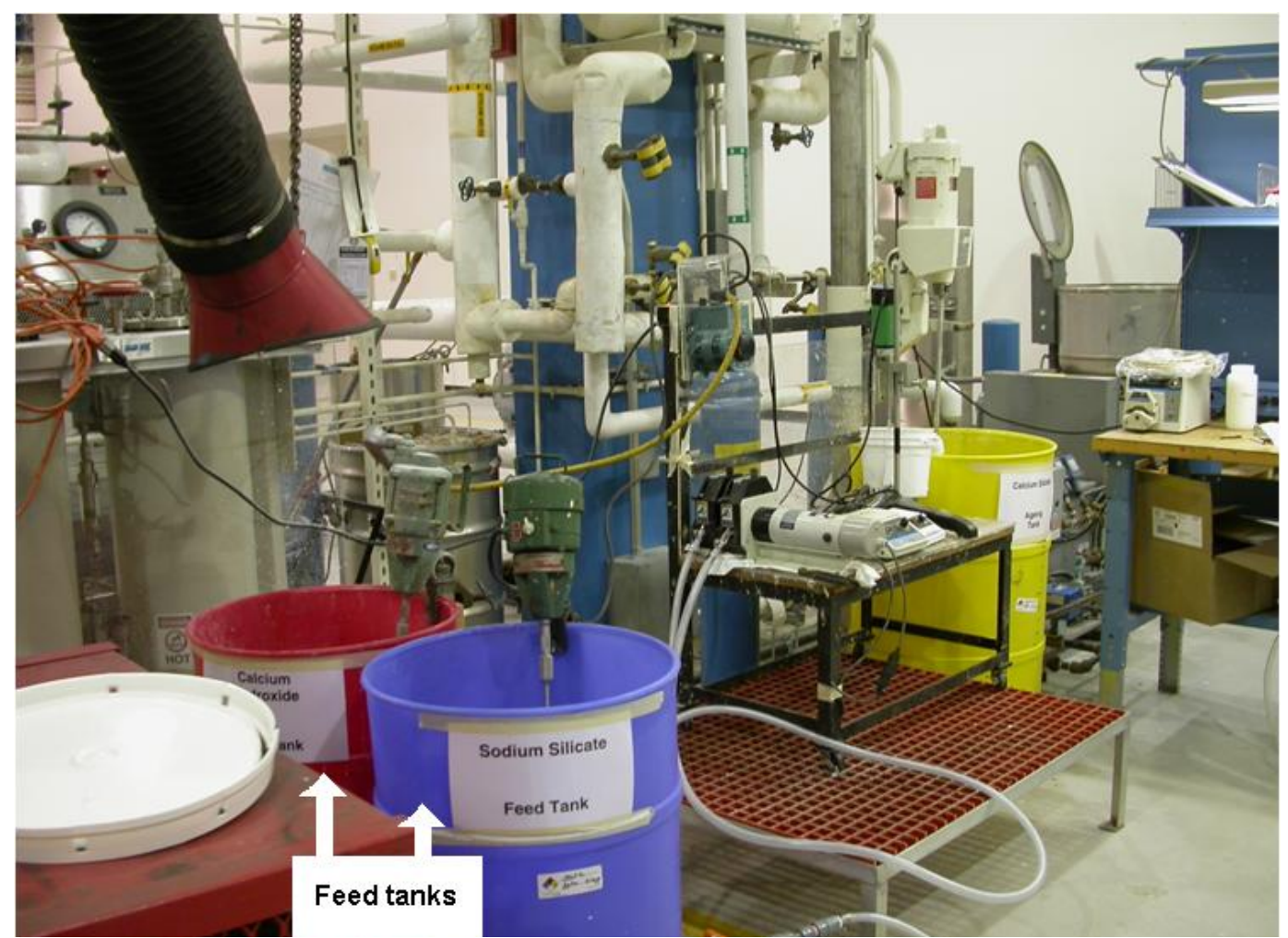

Figure 5.16: Photograph of the pilot plant for the production of nano-structured calcium silicate with feed tanks in the foreground.

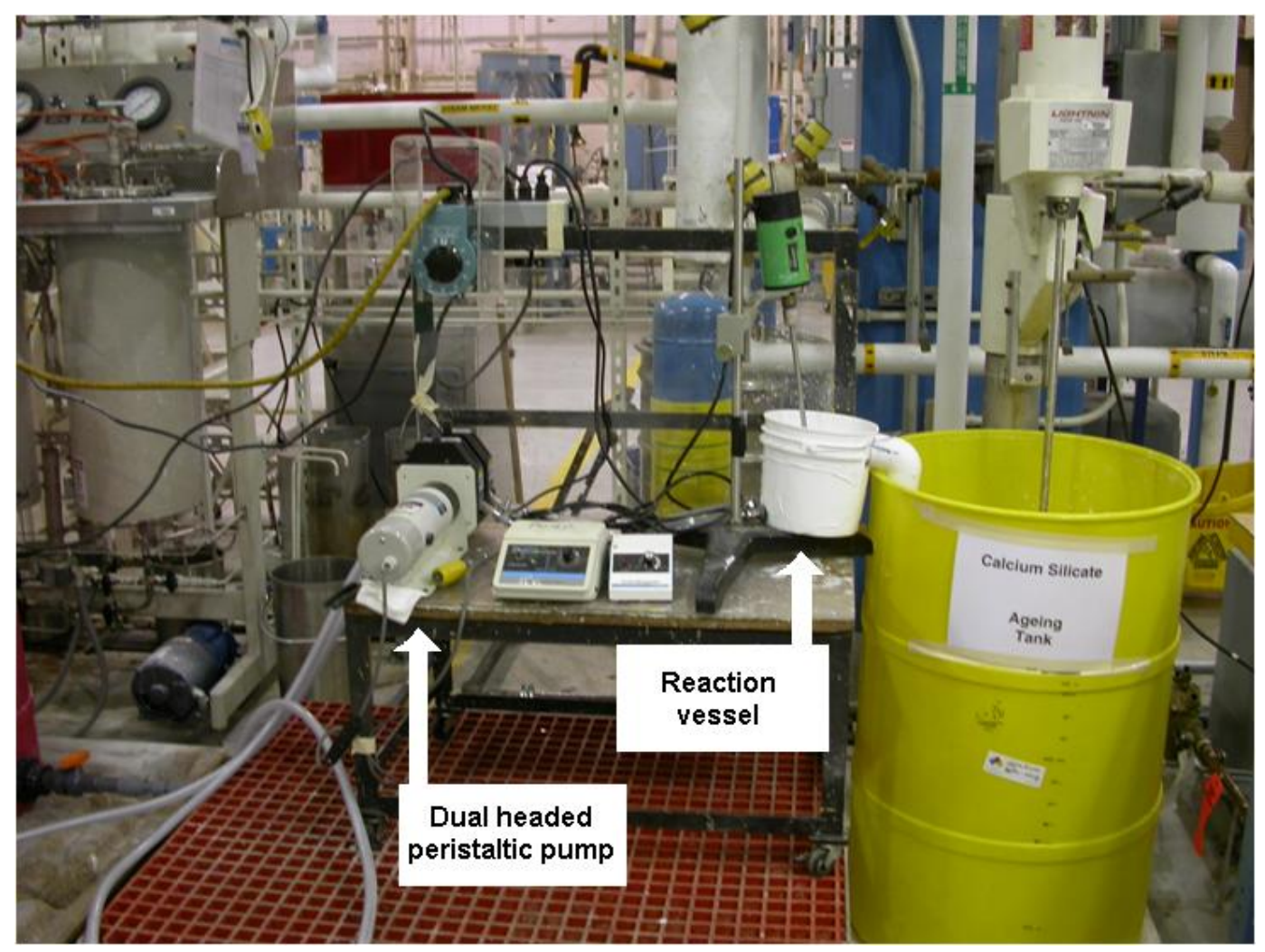

Figure 5.17: Photograph of peristaltic pump, reaction vessel, and ageing tank of pilot plant for the production of nano-structured calcium silicate. 


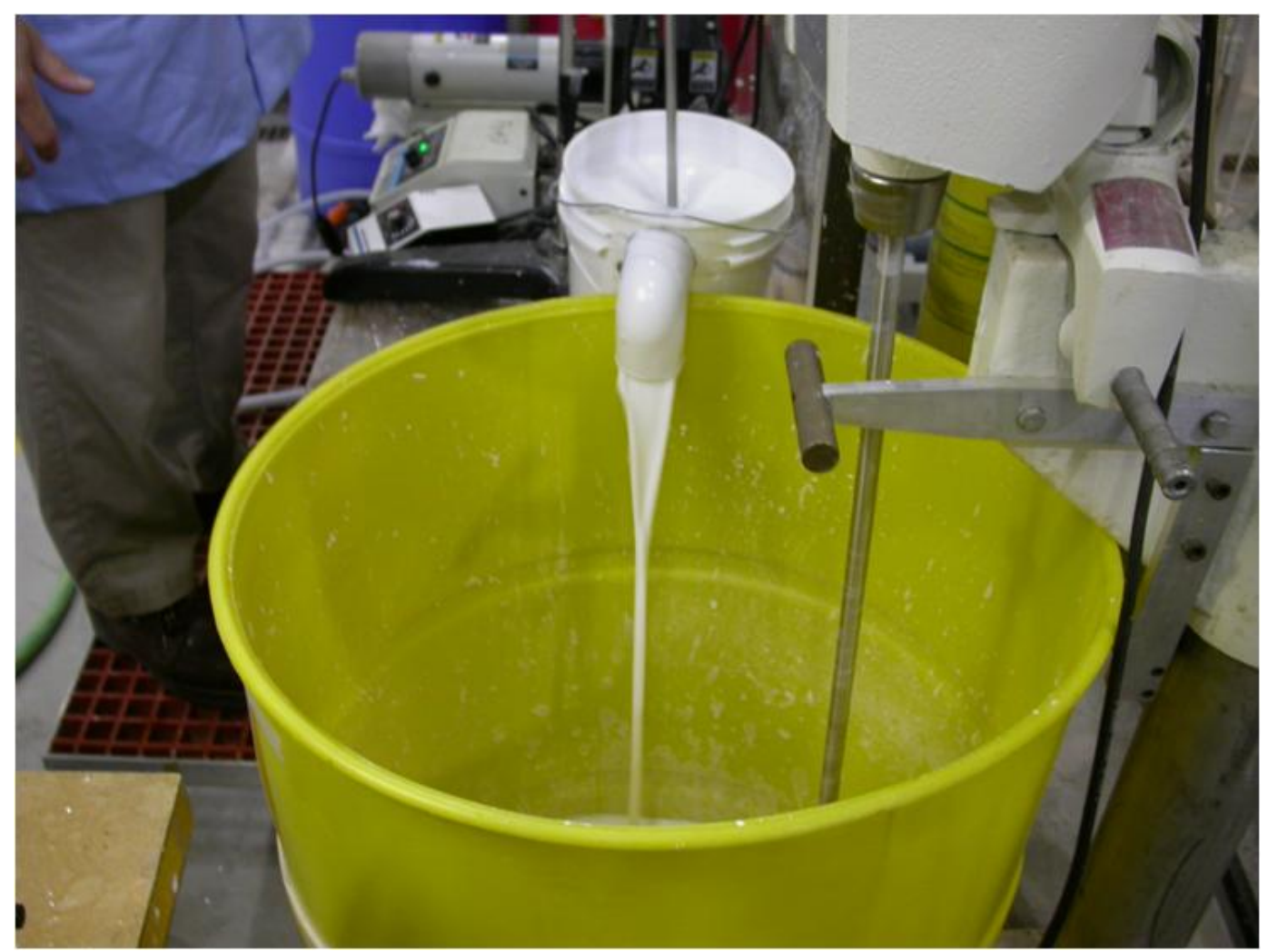

Figure 5.18: Photograph of the pilot plant in operation with the nano-structured calcium silicate slurry over flowing from the reaction vessel into the ageing tank.

One of the main applications envisioned for nano-structured calcium silicate (especially that which has been reinforced) is as a paper filler or in a paper coating. Minerals are added to paper for various reasons, such as to:

- provide cost savings (if the filler is cheaper than pulp)

- increase brightness

- improve optical properties

- improve smoothness

- improve ink retention

- reduce print through

The major disadvantage of filler particles is that they tend to decrease the relative bonded area between adjacent fibres, which usually results in a lower strength of filled paper products compared to unfilled paper. Two of the main properties of common paper fillers are given in Table 5.8. 
Table 5.8: Properties of paper fillers (Hubbe, 2006)

\begin{tabular}{lcc}
\hline Filler type & Brightness, $\%$ & $\begin{array}{c}\text { Surface area } \\
\left(\mathrm{m}^{2} / \mathrm{g}\right)\end{array}$ \\
\hline $\begin{array}{l}\text { calcium carbonate: } \\
\text { ground (GCC) }\end{array}$ & $95-97$ & $2-12$ \\
$\quad$ precipitated (PCC) & $97-99$ & $3-25$ \\
kaolin clay & $80-90$ & $15-25$ \\
titanium dioxide: & & \\
$\quad$ anatase & $98-99$ & $7-12$ \\
$\quad$ rutile & $98-99$ & $7-12$ \\
talc & $70-90$ & $9-20$ \\
synthetic silicates & $93-95$ & $45-75$ \\
\hline
\end{tabular}

The whiteness of fully bleached kraft pulp is 94 (unbleached it is only 15) so any filler that exceeds this value will positively effect the whiteness of the sheet (Smook, 1992). Reinforced nano-structured calcium silicate prepared using technical grade calcium hydroxide has a whiteness of ca. 98. Most of the minerals listed above are added to improve brightness and lack significant porosity as can be seen by their surface areas (that are mainly due to their small particle size rather than internal surfaces). The main inorganic component currently added to paper as a filler because of its porosity are precipitated silicas that have oil absorption capacities of 150 to $350 \mathrm{~g} / 100 \mathrm{~g}$. The potential advantage of reinforced nano-structured calcium silicate is that it has a larger oil absorption capacity than even the best examples of the only material that is used for the improvements gained by porous fillers. Large pore volume materials are able to reduce print through by absorbing ink at the location where it is applied on the sheet. Preliminary tests have been undertaken where the effectiveness of nano-structured calcium silicate as a filler has been compared with calcined clay in 45 and $55 \mathrm{~g} / \mathrm{m}^{2}$ newsprint (Johnston et al., 2004). Nano-structured calcium silicate was able to match the performance of the established calcined clay filler in its ability to increase the opacity of the sheet (Figure 5.20). This shows that the material is able to scatter light effectively presumably due to the large surface of platelets. 

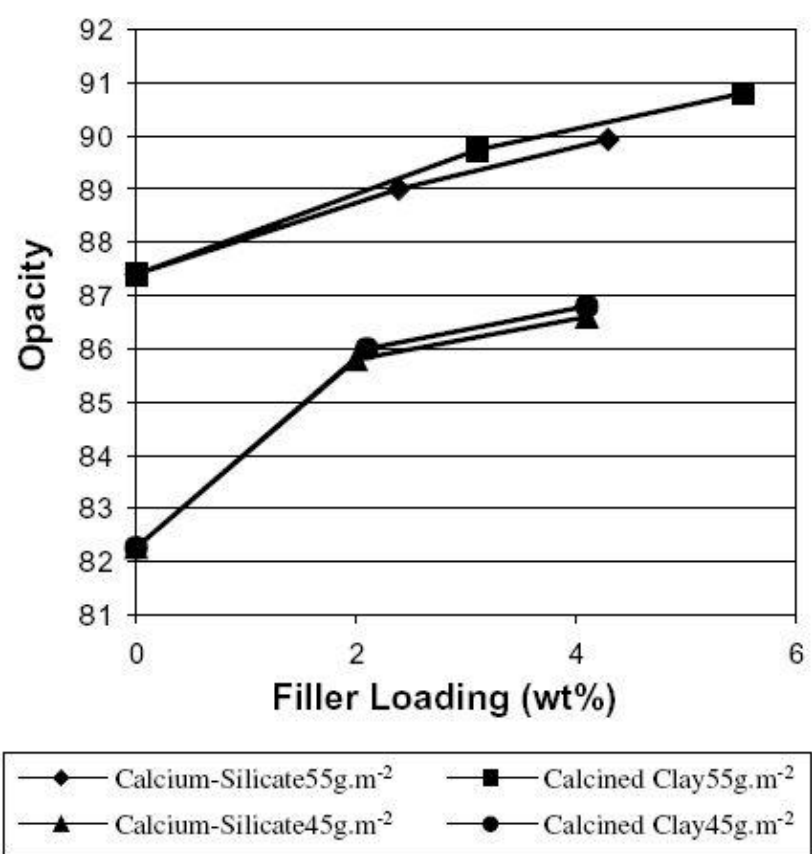

Figure 5.20: The effectiveness of nano-structured calcium silicate for increasing the opacity of newsprint (relative units) (Johnston et al., 2004).
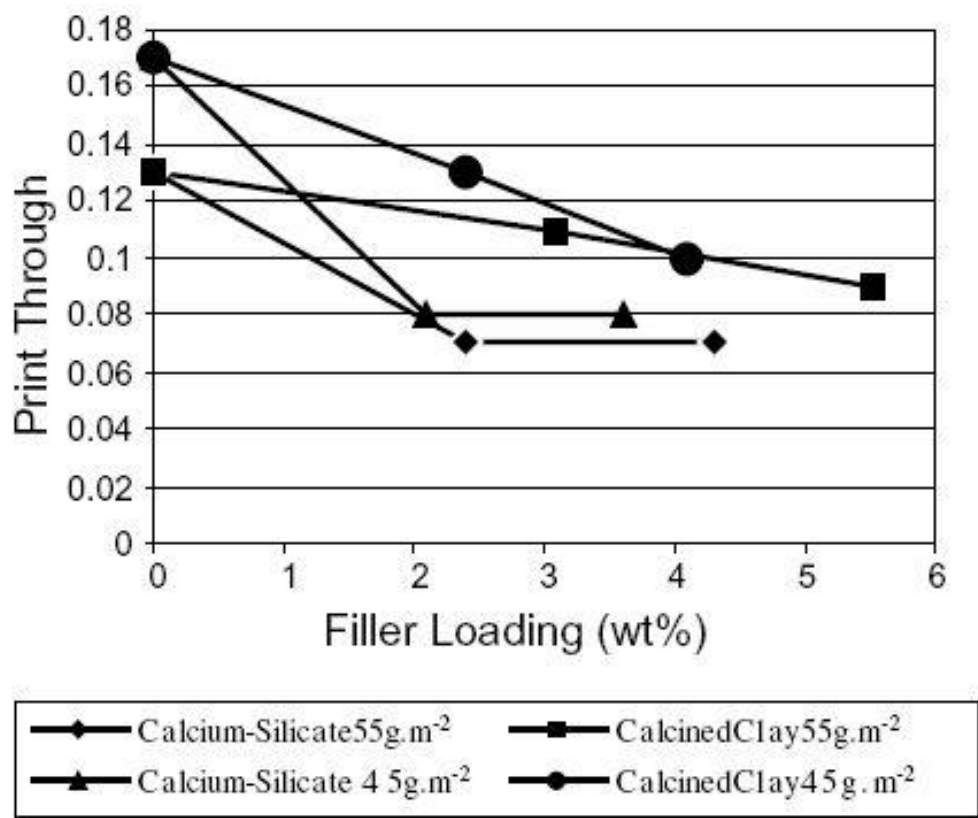

Figure 5.21: The ability of nano-structured calcium silicate to reduce print through (relative units) (Johnston et al., 2004).

The ability of nano-structured calcium silicate to reduce print through due to its porosity is substantially better than that of the calcined clay (Figure 5.21). Print through is the ability to see the print on one side of the sheet from the reverse side. A 2\% loading of calcium silicate was able to reduce the print through of 45 $\mathrm{g} / \mathrm{m}^{2}$ newsprint by $40 \%$ and $55 \mathrm{~g} / \mathrm{m}^{2}$ newsprint by over $50 \%$. The increase in opacity and a brightness of 94 to 95 that is comparable with other fillers means 
that nano-structured calcium silicate can improve the optical properties of the sheet as well as currently used fillers. The combination of being able to excel in improving print quality as well the optical properties indicates that the material has great potential in this application. It is expected that nanostructured calcium silicate could produced for a comparable cost to that of calcined clay fillers, therefore the improvements in sheet performance can be gained without significantly changing the cost of producing the paper. Other research has been undertaken in modifying the surface of nano-structured calcium silicate to improve its dye affinity and therefore ink retention which consequently enhanced the materials performance (Neumann et al., 2005). The ability to perform chemistry on the surface of nano-structured calcium silicates to further improve its properties is as yet essentially untapped and offers the possibility of greater advancements. 


\section{Chapter 6}

\section{NANO-STRUCTURED CALCIUM SILICATE FROM GEOTHERMAL WATER}

Hydrothermal resources occur where a heat source (magma) is close to the earth's surface coincidentally with water that has penetrated fault, fissures or porous regions of hot rock (Figure 6.1). These conditions are met in regions of the world where continental plates meet as the upwelling of magma and the earth's crust lead to areas where the rock temperature is higher than the worldwide average. The water in the resource may be trapped under cap rock as a hot liquid under pressure and can reach temperatures that exceed $300{ }^{\circ} \mathrm{C}$ or more infrequently be present as steam. Surface manifestations of a hydrothermal resource may be visible as geysers, fumaroles, or hot springs, although often there is no indication on the surface of a hydrothermal resource.

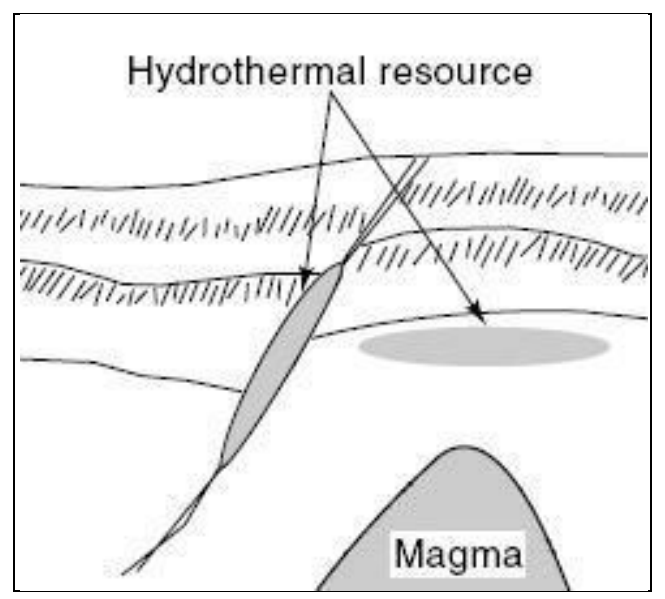

Figure 6.1: Schematic of a hydrothermal resource. 
Most of New Zealand's installed geothermal generating capacity of over 400 MWe (Hall, 2004), is situated in the Taupo Volcanic Zone (Figure 6.2). Within this area geothermal water is used directly as a source of thermal energy and for electricity generation, with the Wairakei resource being the first in the world to use hot geothermal water for electricity generation in 1958.

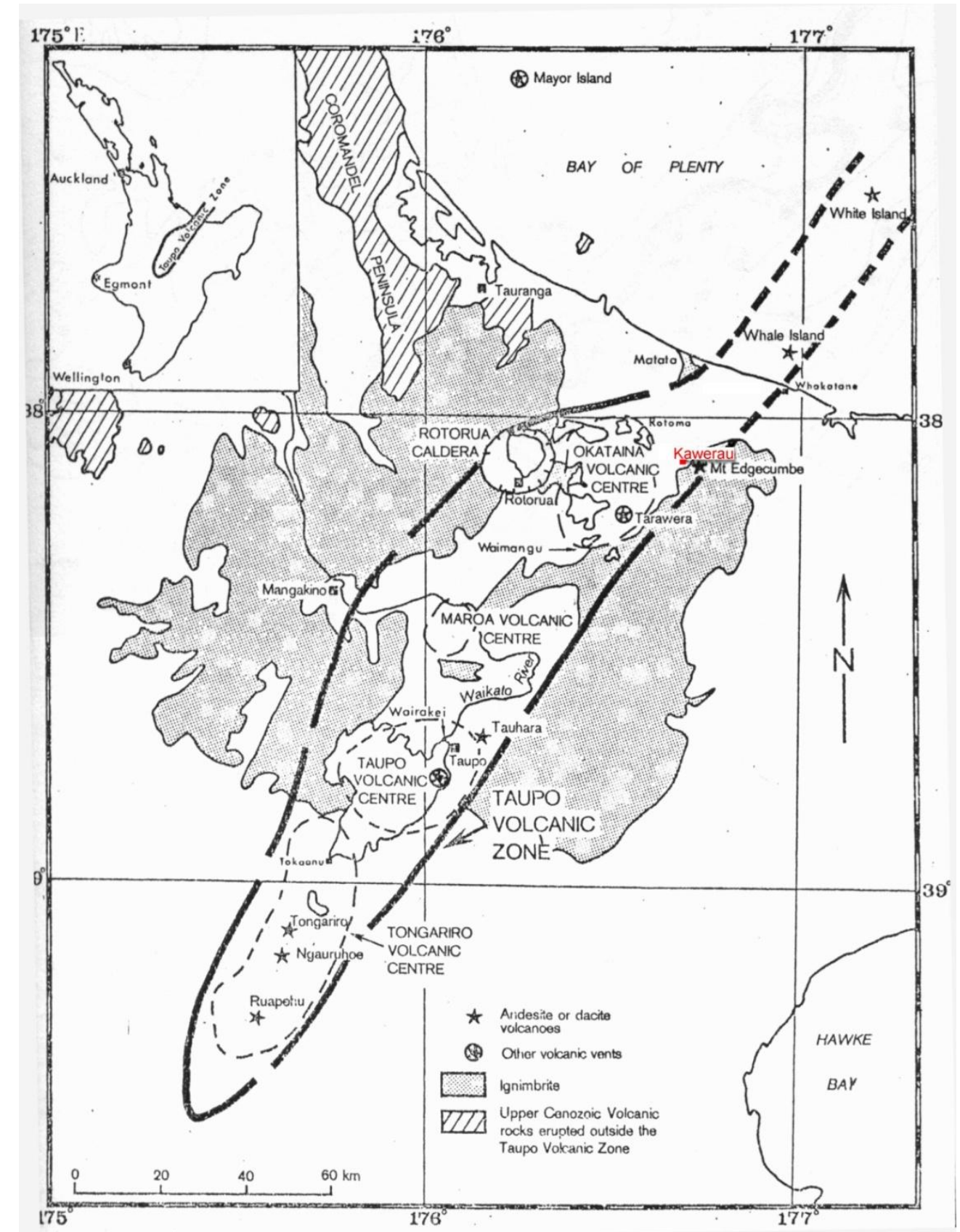

Figure 6.2: The Taupo Volcanic Zone in the central North Island of New Zealand (with Kawerau highlighted). 
Geothermal water in excess of ca. $150{ }^{\circ} \mathrm{C}$ is suitable for electricity production with the two main methods of generation being through the use of flash and binary plants. In flash plants (Figure 6.3) the geothermal water is reduced in pressure in a cyclone separator (B) that separates (flashes) the fluid into vapour and liquid fractions. Approximately $30 \%$ of the total fluid mass flashes off to steam when the pressure is reduced to atmospheric conditions. The steam is then used to drive a turbine (D) to generate electricity, while the liquid is often reinjected ( $\mathrm{C}$ and $\mathrm{E}$ ) back into the reservoir due to either environmental concerns surrounding introducing the water into waterways or to recharge the reservoir and maintain its pressure.

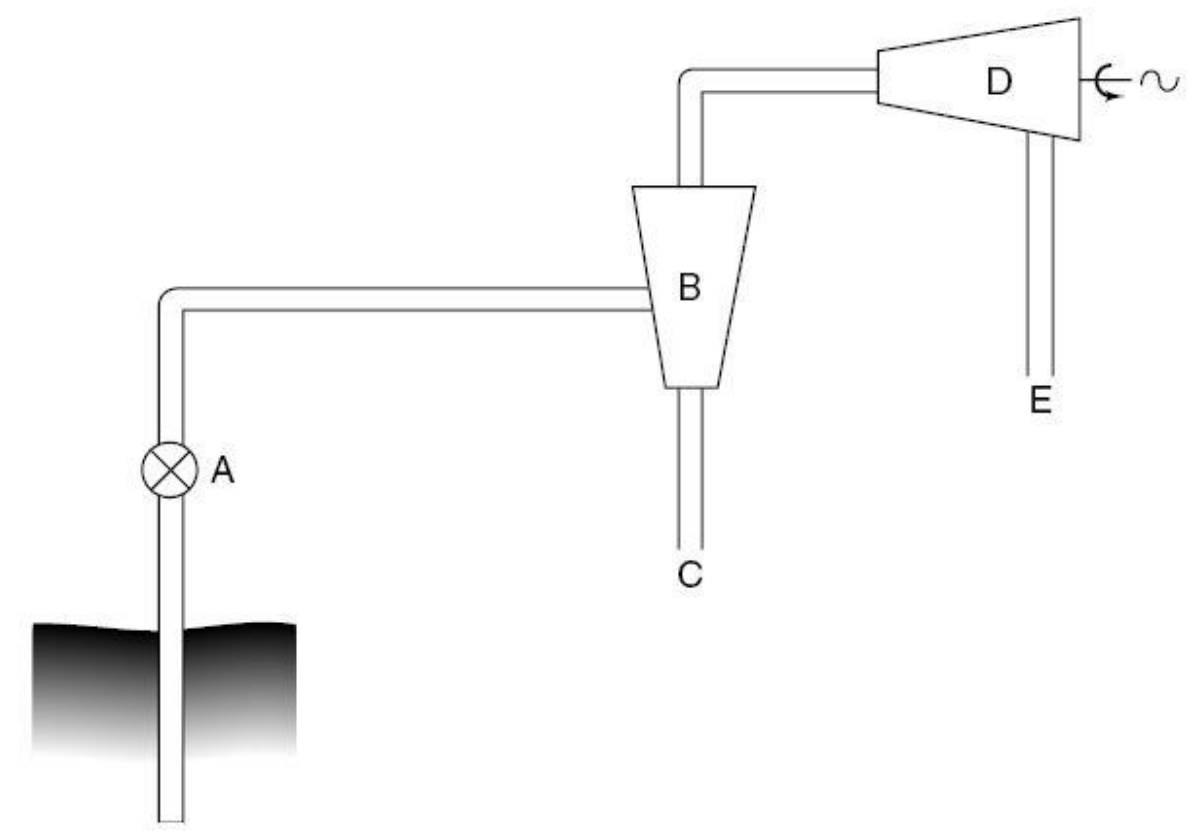

Figure 6.3: Schematic of a flash plant for the generation of electricity form geothermal water (Mink, 2005).

The environmental concerns with geothermal water are usually centred on the temperature of the water and the presence of dissolved species that may be present in supersaturated concentrations. These species are present as a result of the geothermal water dissolving the surrounding rock of the underground reservoir. The concentration of these species is determined by the solubility at the elevated pressures and temperatures inside the reservoir. Each reservoir therefore produces geothermal water with different species that are present in different concentrations depending upon the composition of the rock, and the 
temperature and pressure of the field. Since flash plants operate by separating the geothermal fluid into liquid and vapour, the resulting decrease in volume of the fluid also has the effect of concentrating any species that is present.

Binary plants generate electricity without vaporising the geothermal water (Figure 6.4). Thermal energy from the geothermal water (A) is transferred through a heat exchanger (B) to a volatile low molecular weight hydrocarbon (such as 2-methylpropane) or fluorinated hydrocarbon that is vaporised and used to drive a turbine (D). The vaporised fluid is then recondensed (E) and recirculated to be heated again by further geothermal water. The spent geothermal water can then be reinjected underground as in flash plants.

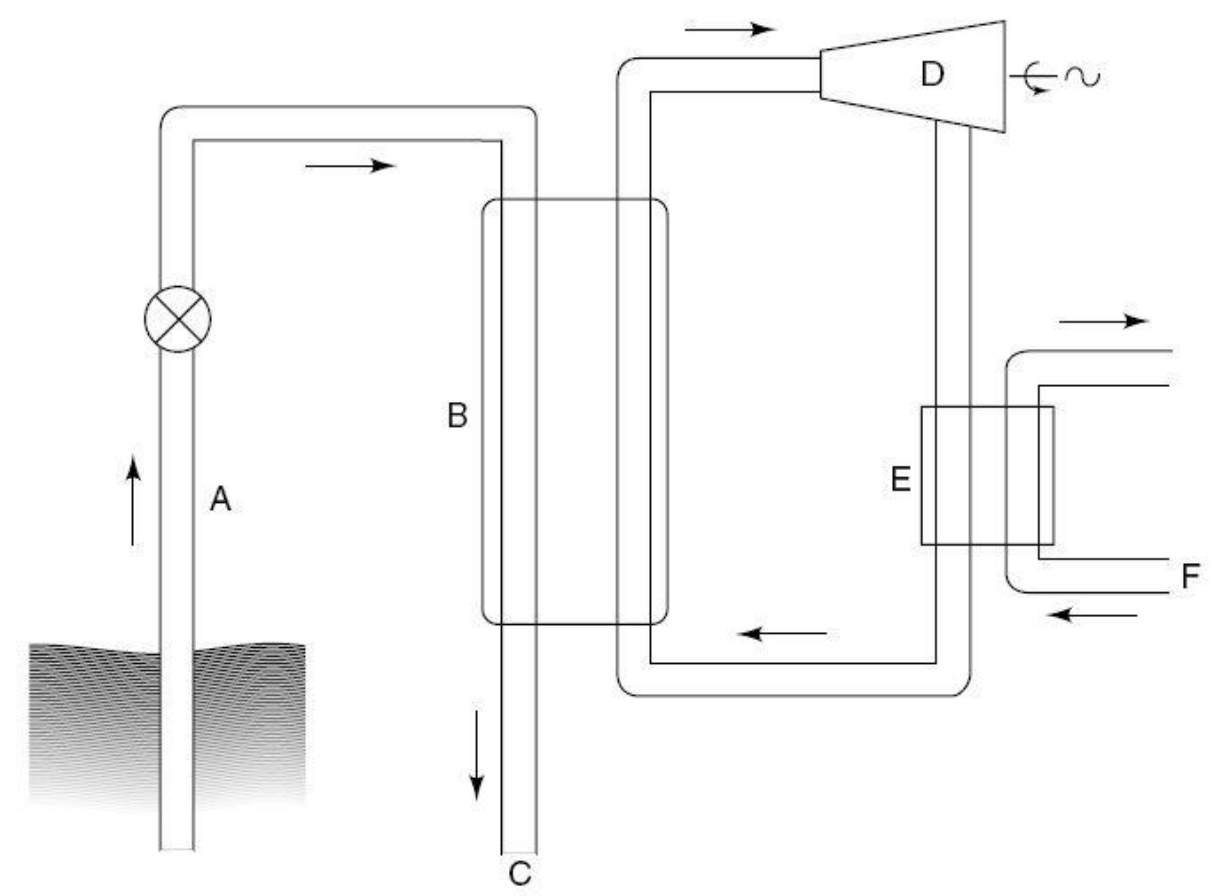

Figure 6.4: Schematic of a binary plant for the generation of electricity form geothermal water (Mink, 2005).

Geothermal energy produces about 7\% of New Zealand's electricity supply (Hall, 2004). With electricity demand and consumption increasing every year and current global emphasis on generating electricity from sources that have little environmental impact, power generation from geothermal energy has become an increasingly attractive option. Geothermal plants produce only ca. 
$5 \%$ as much carbon dioxide and $<1 \%$ of nitrous and sulfur oxides that are emitted from fossil fuel using power plants in generating an equivalent amount of electricity (Mink, 2005). Currently only a fraction of the energy available in geothermal water is utilised due to the problem of deposition of silica scale. For example, the Kawerau field has been ranked as New Zealand's geothermal field that has the greatest potential additional capacity (Table 6.1).

Table 6.1: Existing and potential electricity generation of the Kawerau geothermal field (Hall, 2004)

\begin{tabular}{lcc}
\hline & Current & Potential \\
\hline Installed capacity (MW) & 15 & 372 \\
Annual production (GWh p.a.) & 118 & 2810 \\
\hline
\end{tabular}

However, one of the reasons that the potential is not realised is because wells in the Kawerau field suffer rapid rundown due to mineral deposition. The problem of scale formation arises as a consequence of the decrease in solubility of silica and other minerals as the temperature decreases due to using the thermal energy. The result is a hard coating of silica and mineral scale building up inside of all pipes and channels through which the super-saturated water flows. The current method of alleviating this problem is by not utilising all of the thermal energy of the water and reinjecting the still hot water back underground prior to the solubility decreasing excessively. Despite taking this step to reduce the amount of scale that forms, this does not completely remove the problem and therefore the costly process of removing the scale must still be undertaken.

Another method that has been used to prevent the build up of scale is by precipitating silica from the geothermal water in a controlled fashion. This has the effect of reducing the monomeric silica concentration in solution to solubility in the given conditions, therefore greatly reducing the build-up of scale and allowing more thermal energy to be utilised from the water, but also forming a useful material that has a commercial value. The precipitation of nano-structured calcium silicate from geothermal water provides potential advantages over the formation of precipitated silica not only in terms of the 
improved physical characteristics of the material, but also from the nature of the reaction and how the material is prepared. The formation of precipitated silica from solution takes place through the polymerisation of monomeric silica that results in sol particles as discussed in Section 1.3 (Harper, 1994; Harper et al., 1997; Meyer, 1996). The stable sol can be destabilised by the addition of a salt which results in the precipitation of silica with a network structure. The polymerisation reaction uses only the monomeric silica above the equilibrium solubility with the remaining monomeric silica staying in solution. The precipitation of nano-structured calcium silicate using sodium silicate however, has been found in this work to essentially remove all monomeric silica from solution when the calcium concentration is greater than the monomeric silica concentration (Section 3.1). This would therefore effectively remove the possibility of any silica scale forming and allow all of the thermal energy of the geothermal water to be utilised. The ability to remove all of the monomeric silica from solution also allows for the production of nano-structured calcium silicate from geothermal water with low monomeric silica concentrations at or below the solubility. The removal of all monomeric silica from solution also means that the amount of product formed per unit volume of geothermal water is greater.

Another potential advantage of precipitating nano-structured calcium silicate is that the precipitation step occurs at the very start of the reaction. The formation of precipitated silica requires a lengthy polymerisation process prior to precipitation. This therefore requires for a large volume of geothermal water to be stored during the polymerisation process before the precipitation reaction and subsequent reduction of the reaction volume by passing the slurry through a clarifier. Despite the ageing process of nano-structured calcium silicate taking longer than the polymerisation of silica, because the solids are precipitated out at the start of the reaction, the slurry may be reduced in volume at this early stage (as the solids make up less than $10 \%$ of the volume). As the concentration of monomeric silica in geothermal water is so low, the nano-structured calcium silicate slurry when allowed to settle has a 
supernatant that makes up $>90 \%$ of the volume. The slurry could therefore be run into a settling tank and the supernatant discarded and consequently reduce the volume of vessel required per unit weight of material produced.

\subsection{The first series of reactions using geothermal water}

With the potential advantages of precipitating a nano-structured calcium silicate rather than silica, a study was carried out to try to determine the optimum conditions for forming a nano-structured calcium silicate with a large oil absorption capacity and a high surface area. However, at this stage of the programme the methods to prevent collapse of the structure had not yet been developed. Therefore all samples were not treated in any way before drying at $110{ }^{\circ} \mathrm{C}$. As the experiments were carried out in the field where no analytical methods were available, a wide range of $\mathrm{pH}$ and calcium ion concentrations were used.

The experiments were carried out in Kawerau beside the Tarawera River using geothermal water from a binary generation plant. The Kawerau geothermal field has five production wells that remove ca. 1100 tonnes of fluid per hour from the reservoir. The geothermal water that exceeds the reinjection well capacity is piped from the plant to a silencer where the fluid was released to atmospheric pressure and flowed over a weirbox down a channel to a cooling lagoon (Figure 6.5 and Figure 6.6). 


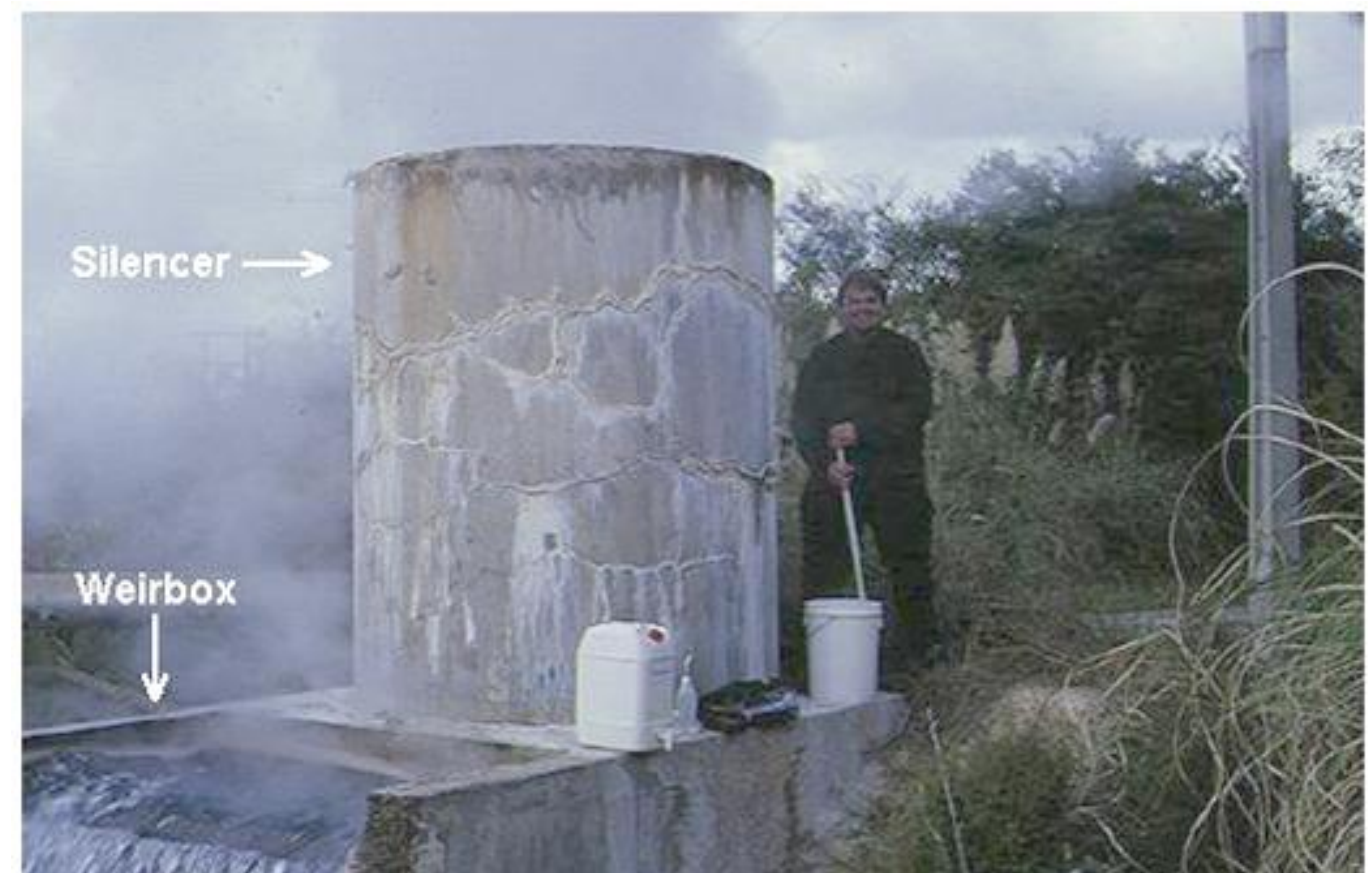

Figure 6.5: Source of geothermal water used in Kawerau

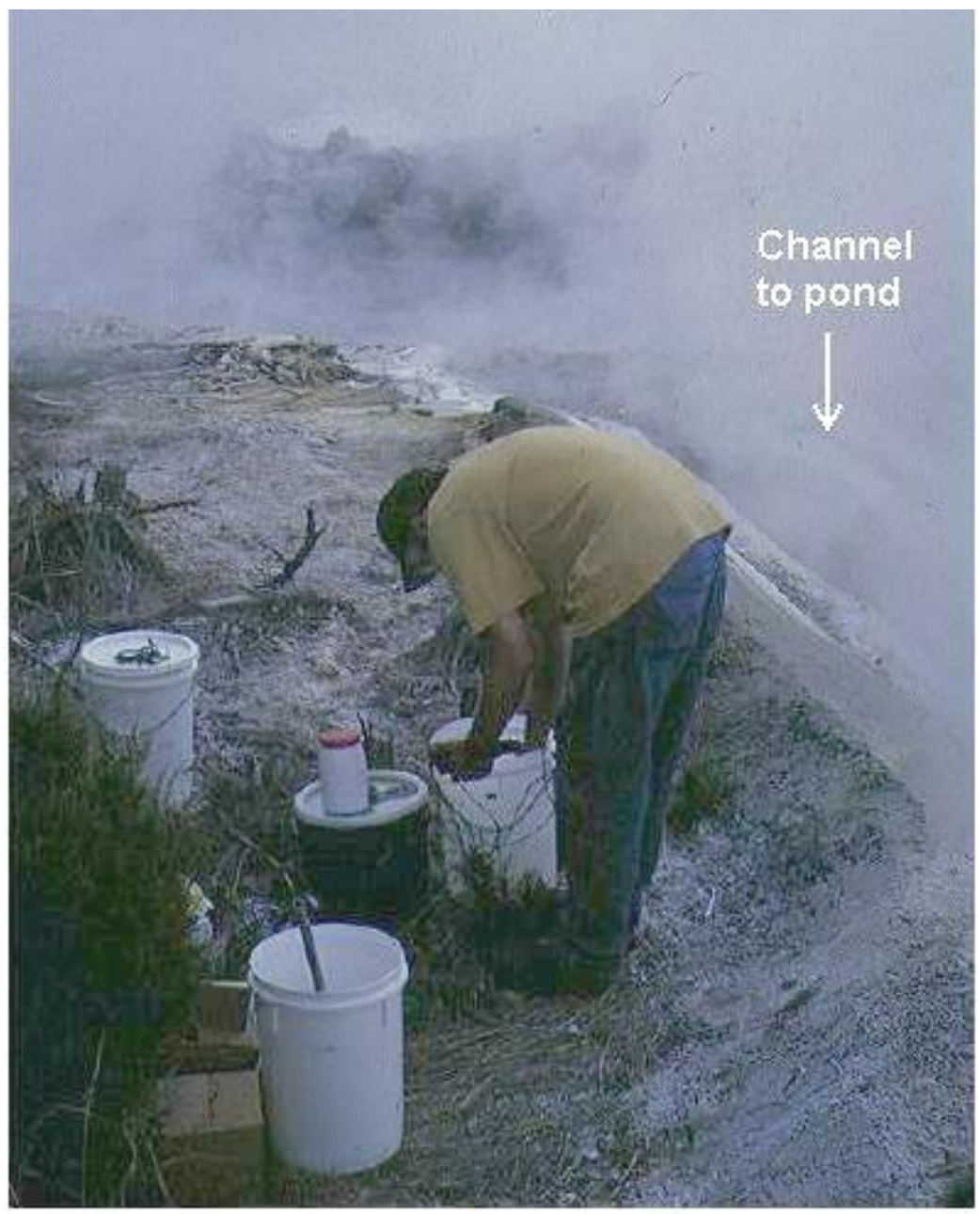

Figure 6.6: Work area beside weirbox at Kawerau. 
The geothermal water from deeper wells in this field typically has a temperature of 300 to $315{ }^{\circ} \mathrm{C}$ and a dissolved carbon dioxide content of ca. $1.5 \%$, which is higher than that of other fields such as a Wairakei. The $\mathrm{pH}$ range of the geothermal water from different wells varies from 7.6 to 9.2 and is governed by fluid - mineral equilibria and the concentration of the dissolved acidic gases carbon dioxide and hydrogen sulfide. The pH of water from binary plants is typically lower than that of flashed water because of the higher concentration of the dissolved gases present. The $\mathrm{pH}$ of the reservoir has been calculated to be ca. 6.3, which increases upon steam separation as most of the dissolved carbon dioxide is lost from the water phase. A detailed analysis of the chemical composition of geothermal water from different wells in the late eighties is given by Harper (1994).

Geothermal water that was at ca. $100{ }^{\circ} \mathrm{C}$ after just being flashed to atmospheric pressure was collected by submerging a $20 \mathrm{~L}$ bucket into the weirbox and decanting off the excess to a volume of $10 \mathrm{~L}$. The reaction was then undertaken as quickly as possible to reduce the time in which the polymerisation of silica could occur and therefore avoid forming sol particles. The geothermal water was rapidly stirred by hand with a large stirring rod as calcium hydroxide was added and immediately followed by a volume of $25 \% \mathrm{w} / \mathrm{w}$ sodium hydroxide via a syringe. As the geothermal water is more acidic than a sodium silicate solution, it was necessary to add sodium hydroxide in some cases as the calcium hydroxide alone did not sufficiently raise the $\mathrm{pH}$ into the range required. All of the reactions were stirred for 15 minutes following the addition of calcium hydroxide and then left aside to settle. The supernatant ( $>9$ litres) was then decanted off and the remaining slurry stored in a $1 \mathrm{~L}$ plastic container for analysis later in the laboratory. Samples of the solution were collected during the reaction after being passed through a $0.65 \mu \mathrm{m}$ filter into a $20 \mathrm{~mL}$ plastic bottle that contained two drops of concentrated hydrochloric to prevent further polymerisation of silica by acidifying the solution. Details of the reactions to produce nano-structure calcium silicate from geothermal water at Kawerau are given in Table 6.2, and Table 6.3. 
CHAPTER 6: NANO-STRUCTURED CALCIUM SILICATE FROM GEOTHERMAL WATER

Table 6.2: Details of reactions using geothermal water without the addition of sodium hydroxide during the first series of work at Kawerau

\begin{tabular}{|c|c|c|c|c|c|c|c|c|}
\hline $\begin{array}{c}\text { Sample } \\
\text { name }\end{array}$ & $\begin{array}{c}\text { Calcium } \\
\text { added } \\
(\mathrm{mg} / \mathrm{L})\end{array}$ & $\begin{array}{l}\text { Time } \\
(\mathrm{min})\end{array}$ & $\mathrm{pH}$ & $\begin{array}{c}\text { Temp. } \\
\left({ }^{\circ} \mathrm{C}\right)\end{array}$ & $\begin{array}{l}{\left[\mathrm{SiO}_{2}\right]_{\mathrm{m}}} \\
(\mathrm{mg} / \mathrm{L})\end{array}$ & $\begin{array}{c}\text { Product } \\
\text { weight } \\
\text { (g) }\end{array}$ & $\begin{array}{c}\text { Oil } \\
\text { absorption } \\
\text { (g/100g) }\end{array}$ & $\begin{array}{c}\text { Surface } \\
\text { area } \\
\left(\mathrm{m}^{2} / \mathrm{g}\right)\end{array}$ \\
\hline \multirow[t]{3}{*}{ K2 } & 500 & 2 & 9.03 & 91.6 & 457 & & & \\
\hline & & 5 & 9.26 & 86.4 & 438 & & & \\
\hline & & 15 & 9.42 & 77.4 & 413 & 9.06 & 157 & 33 \\
\hline \multirow[t]{3}{*}{ K3 } & 600 & 2 & 9.23 & 89.5 & & & & \\
\hline & & 5 & 9.29 & 85.5 & 417 & & & \\
\hline & & 15 & 9.48 & 77.5 & 121 & 11.05 & 167 & 38 \\
\hline \multirow[t]{3}{*}{ K4 } & 700 & 2 & 9.12 & 88.4 & & & & \\
\hline & & 5 & 9.22 & 84.4 & 380 & & & \\
\hline & & 15 & 9.41 & 75.0 & 120 & 12.47 & 160 & 38 \\
\hline \multirow[t]{4}{*}{ K5 } & 800 & 1 & 9.22 & 90.3 & & & & \\
\hline & & 2 & 9.31 & 89.2 & & & & \\
\hline & & 5 & 9.37 & 86.5 & 399 & & & \\
\hline & & 15 & 9.54 & 78.6 & 115 & 14.90 & 161 & 37 \\
\hline \multirow[t]{3}{*}{ K6 } & 900 & 2 & 9.25 & 86.8 & & & & \\
\hline & & 5 & 9.36 & 79.7 & 117 & & & \\
\hline & & 15 & 9.49 & 74.5 & 112 & 17.08 & 147 & 40 \\
\hline \multirow[t]{4}{*}{ K7 } & 1000 & 2 & 9.44 & 89.3 & & & & \\
\hline & & 5 & 9.49 & 86.2 & 111 & & & \\
\hline & & 15 & 9.75 & 78.1 & & & & \\
\hline & & 20 & & & 304 & 19.22 & 165 & 44 \\
\hline \multirow[t]{4}{*}{ K8 } & 1100 & 1 & 9.42 & 90.5 & & & & \\
\hline & & 2.5 & 9.40 & 88.4 & & & & \\
\hline & & 6 & 9.57 & 84.6 & 360 & & & \\
\hline & & 15 & 9.87 & 77.2 & 253 & 21.91 & 173 & 55 \\
\hline \multirow[t]{3}{*}{ K9 } & 1200 & 2 & 9.35 & 88.7 & & & & \\
\hline & & 5 & 9.40 & 85.2 & 206 & & & \\
\hline & & 15 & 9.65 & 77.0 & 294 & 23.14 & 144 & 43 \\
\hline \multirow[t]{3}{*}{ K10 } & 1500 & 2 & 9.65 & 90.2 & & & & \\
\hline & & 5 & 9.71 & 83.6 & 268 & & & \\
\hline & & 15 & 9.97 & 77.3 & 148 & 29.24 & 165 & 46 \\
\hline \multirow[t]{3}{*}{ K11 } & 2000 & 2 & 9.90 & 86.9 & & & & \\
\hline & & 5 & 10.13 & 84.0 & 62 & & & \\
\hline & & 15 & 10.79 & 74.2 & 7 & 35.49 & 172 & 55 \\
\hline \multirow[t]{4}{*}{ K12 } & 1800 & 1.5 & 9.71 & 91.1 & & & & \\
\hline & & 2 & 9.67 & 90.2 & & & & \\
\hline & & 5 & 9.78 & 85.8 & 235 & & & \\
\hline & & 15 & 10.38 & 76.4 & 91 & 35.83 & 177 & 51 \\
\hline
\end{tabular}


Table 6.3: Details of reactions with the addition of sodium hydroxide for $\mathrm{pH}$ adjustment using geothermal water during the first series of work at Kawerau

\begin{tabular}{|c|c|c|c|c|c|c|c|c|c|}
\hline $\begin{array}{l}\text { Sample } \\
\text { name }\end{array}$ & $\begin{array}{c}\text { Calcium } \\
\text { added } \\
(\mathrm{mg} / \mathrm{L})\end{array}$ & $\begin{array}{l}\text { Vol. 25\% } \\
\mathrm{NaOH}(\mathrm{mL})\end{array}$ & $\begin{array}{l}\text { Time } \\
\text { (min) }\end{array}$ & $\mathrm{pH}$ & Temp. $\left({ }^{\circ} \mathrm{C}\right)$ & $\begin{array}{l}{\left[\mathrm{SiO}_{2}\right]_{\mathrm{m}}} \\
(\mathrm{mg} / \mathrm{L})\end{array}$ & $\begin{array}{c}\text { Product } \\
\text { weight }(\mathrm{g})\end{array}$ & $\begin{array}{l}\text { Oil absorption } \\
\text { (g/100g) }\end{array}$ & $\begin{array}{l}\text { Surface area } \\
\qquad\left(\mathrm{m}^{2} / \mathrm{g}\right)\end{array}$ \\
\hline \multirow[t]{4}{*}{ K13 } & 1000 & 20 & 1 & 10.19 & 92.4 & & & & \\
\hline & & & 2 & 10.27 & 89.5 & & & & \\
\hline & & & 5 & 10.40 & & 320 & & & \\
\hline & & & 15 & 10.62 & 78.5 & 133 & 23.63 & 207 & 34 \\
\hline \multirow[t]{4}{*}{ K14 } & 1000 & 30 & 1 & 10.39 & 92.5 & & & & \\
\hline & & & 2 & 10.49 & 90.1 & & & & \\
\hline & & & 5 & 10.57 & 87.6 & 285 & & & \\
\hline & & & 15 & 10.76 & 77.9 & 194 & 21.89 & 198 & 58 \\
\hline \multirow[t]{4}{*}{ K15 } & 1000 & 15 & 1 & 9.99 & 92.5 & & & & \\
\hline & & & 2 & 10.01 & 91.2 & & & & \\
\hline & & & 5 & 10.17 & 88.0 & 370 & & & \\
\hline & & & 15 & 10.45 & 78.4 & 235 & 21.60 & 207 & 44 \\
\hline \multirow[t]{4}{*}{ K16 } & 1500 & 20 & 1 & 10.07 & 94.0 & & & & \\
\hline & & & 2 & 10.25 & 90.0 & & & & \\
\hline & & & 5 & 10.35 & 86.1 & 302 & & & \\
\hline & & & 15 & 10.65 & 79.0 & 136 & 31.85 & 183 & 54 \\
\hline \multirow[t]{4}{*}{ K17 } & 1500 & 10 & 1 & 9.74 & 93.8 & & & & \\
\hline & & & 2 & 9.85 & 91.2 & & & & \\
\hline & & & 5 & 9.94 & 88.0 & 277 & & & \\
\hline & & & 15 & 10.33 & 79.1 & 163 & 31.22 & 199 & 52 \\
\hline \multirow[t]{4}{*}{ K18 } & 1500 & 30 & 1 & 10.46 & 91.8 & & & & \\
\hline & & & 2 & 10.51 & 89.5 & & & & \\
\hline & & & 5 & 10.62 & 85.6 & 133 & & & \\
\hline & & & 15 & 10.88 & 75.7 & 38 & 31.96 & 184 & 50 \\
\hline
\end{tabular}




\begin{tabular}{|c|c|c|c|c|c|c|c|c|c|}
\hline $\begin{array}{l}\text { Sample } \\
\text { name }\end{array}$ & $\begin{array}{c}\text { Calcium } \\
\text { added } \\
\text { (mg/L) }\end{array}$ & $\begin{array}{c}\text { Vol. 25\% } \\
\mathrm{NaOH}(\mathrm{mL})\end{array}$ & $\begin{array}{l}\text { Time } \\
(\min )\end{array}$ & $\mathrm{pH}$ & Temp. $\left({ }^{\circ} \mathrm{C}\right)$ & $\begin{array}{l}{\left[\mathrm{SiO}_{2}\right]_{\mathrm{m}}} \\
(\mathrm{mg} / \mathrm{L})\end{array}$ & $\begin{array}{c}\text { Product } \\
\text { weight (g) }\end{array}$ & $\begin{array}{l}\text { Oil absorption } \\
\text { (g/100 g) }\end{array}$ & $\begin{array}{c}\text { Surface area } \\
\left(\mathrm{m}^{2} / \mathrm{g}\right)\end{array}$ \\
\hline \multirow[t]{4}{*}{ K19 } & 800 & 20 & 1 & 10.20 & 91.2 & & & & \\
\hline & & & 2 & 10.17 & 89.2 & & & & \\
\hline & & & 5 & 10.31 & 84.5 & 322 & & & \\
\hline & & & 15 & 10.62 & 72.7 & 68 & 19.08 & 256 & 49 \\
\hline \multirow[t]{4}{*}{ K20 } & 800 & 30 & 1 & 10.33 & 91.4 & & & & \\
\hline & & & 2 & 10.35 & 89.4 & & & & \\
\hline & & & 5 & 10.44 & 86.0 & 372 & & & \\
\hline & & & 15 & 10.71 & 76.2 & 138 & 17.65 & 199 & 46 \\
\hline \multirow[t]{4}{*}{ K21 } & 1200 & 10 & 1 & 9.75 & 92.6 & & & & \\
\hline & & & 2 & 9.75 & 90.9 & & & & \\
\hline & & & 5 & 9.90 & 86.8 & 323 & & & \\
\hline & & & 15 & 10.13 & 76.7 & 271 & 24.08 & 156 & 46 \\
\hline \multirow[t]{4}{*}{ K22 } & 1200 & 20 & 1 & 10.06 & 92.5 & & & & \\
\hline & & & 2 & 10.04 & 91.0 & & & & \\
\hline & & & 5 & 10.17 & 86.5 & 351 & & & \\
\hline & & & 15 & 10.53 & 76.5 & 190 & 26.28 & 201 & 51 \\
\hline \multirow[t]{4}{*}{ K23 } & 1200 & 30 & 1 & 10.29 & 91.7 & & & & \\
\hline & & & 2 & 10.32 & 90.2 & & & & \\
\hline & & & 5 & 10.41 & 86.5 & 308 & & & \\
\hline & & & 15 & 10.65 & 77.7 & 210 & 25.61 & 189 & 57 \\
\hline \multirow[t]{4}{*}{ K24 } & 1800 & 10 & 1 & 9.90 & 92.0 & & & & \\
\hline & & & 2 & 9.90 & 90.5 & & & & \\
\hline & & & 5 & 10.01 & 86.9 & 217 & & & \\
\hline & & & 15 & 10.48 & 76.5 & 84 & 36.11 & 187 & 59 \\
\hline
\end{tabular}




\begin{tabular}{|c|c|c|c|c|c|c|c|c|c|}
\hline $\begin{array}{c}\text { Sample } \\
\text { name }\end{array}$ & $\begin{array}{c}\text { Calcium } \\
\text { added } \\
(\mathrm{mg} / \mathrm{L})\end{array}$ & $\begin{array}{c}\text { Vol. 25\% } \\
\text { NaOH (mL) }\end{array}$ & $\begin{array}{l}\text { Time } \\
(\min )\end{array}$ & $\mathrm{pH}$ & Temp. $\left({ }^{\circ} \mathrm{C}\right)$ & $\begin{array}{l}{\left[\mathrm{SiO}_{2}\right]_{\mathrm{m}}} \\
(\mathrm{mg} / \mathrm{L})\end{array}$ & $\begin{array}{c}\text { Product } \\
\text { weight (g) }\end{array}$ & $\begin{array}{l}\text { Oil absorption } \\
\text { (g/100g) }\end{array}$ & $\begin{array}{c}\text { Surface area } \\
\left(\mathrm{m}^{2} / \mathrm{g}\right)\end{array}$ \\
\hline \multirow[t]{3}{*}{ K25 } & 1800 & 20 & 2 & 10.32 & 88.7 & & & & \\
\hline & & & 5 & 10.45 & 85.7 & 160 & & & \\
\hline & & & 15 & 10.83 & 74.5 & 17 & 37.25 & 179 & 52 \\
\hline \multirow[t]{3}{*}{ K26 } & 1800 & 30 & 2 & 10.37 & 89.8 & & & & \\
\hline & & & 5 & 10.46 & 86.4 & 189 & & & \\
\hline & & & 15 & 10.77 & 77.2 & 64 & 38.31 & 182 & 43 \\
\hline \multirow[t]{3}{*}{ K27 } & 1000 & 20 & 2 & 10.10 & 90.0 & & & & \\
\hline & & & 5 & 10.15 & 85.8 & 259 & & & \\
\hline & & & 15 & 10.45 & 76.6 & 309 & 21.31 & 184 & 44 \\
\hline \multirow[t]{3}{*}{ K28 } & 1000 & 20 & 3 & 10.29 & 87.3 & & & & \\
\hline & & & 5 & 10.39 & 83.9 & 256 & & & \\
\hline & & & 15 & 10.70 & 74.5 & 52 & 23.63 & 208 & 39 \\
\hline \multirow[t]{3}{*}{ K29 } & 600 & 20 & 2 & 10.15 & 88.5 & & & & \\
\hline & & & 5 & 10.27 & 83.2 & 403 & & & \\
\hline & & & 15 & 10.50 & 73.4 & 319 & 12.62 & 194 & 38 \\
\hline \multirow[t]{3}{*}{ K30 } & 600 & 30 & 2.5 & 10.35 & 87.5 & & & & \\
\hline & & & 5 & 10.42 & 84.7 & 443 & & & \\
\hline & & & 15 & 10.68 & 74.5 & 313 & 14.15 & 195 & 43 \\
\hline
\end{tabular}


Analysis of the solids formed using X-ray fluorescence spectroscopy to determine the composition showed that all the materials produced had $\mathrm{Ca} / \mathrm{Si}$ ratios higher than expected (Table 6.5). A summary of the minor components present of all samples prepared are given in (Table 6.4). Based on experiments in the laboratory using sodium silicate, a $\mathrm{Ca} / \mathrm{Si}$ ratio of around 1 is typical for producing a nano-structured calcium silicate with a maximised oil absorption capacity. The $\mathrm{Ca} / \mathrm{Si}$ ratio of 2.5 for the sample prepared with the lowest calcium concentration of $500 \mathrm{mg} / \mathrm{L}$ (K2) was much higher than expected as the monomeric silica concentration in the geothermal water was estimated to be approximately 600 to $800 \mathrm{mg} / \mathrm{L}$. Studying the material with electron microscopes revealed a significant amount of crystalline material that X-ray powder diffraction showed was calcium carbonate and unreacted calcium hydroxide (Figure 6.7 and Figure 6.8). Subsequent Leco analysis was used to determine the proportion of carbon present in the samples. On the assumption that all of the carbon present was from calcium carbonate, the calcium carbonate content was calculated allowing the $\mathrm{Ca} / \mathrm{Si}$ ratio of the remaining calcium silicate to be recalculated. These calculations suggest that a staggeringly large proportion (from 32 to $56 \%$, w/w) of the material precipitated is calcium carbonate which would lower the oil absorption and surface area of the materials as the calcium carbonate is not significantly porous.

Table 6.4: The proportion of minor components present for all samples prepared using geothermal water from the Kawerau field as determined by XRF spectroscopy.

\begin{tabular}{cc}
\hline Component & \% range \\
\hline $\mathrm{TiO}_{2}$ & $0.04-0.07$ \\
$\mathrm{Al}_{2} \mathrm{O}_{3}$ & $0.11-0.34$ \\
$\mathrm{Fe}_{2} \mathrm{O}_{3}$ & $0.01-0.07$ \\
$\mathrm{MnO}$ & $0.00-0.01$ \\
$\mathrm{MgO}$ & $0.82-1.27$ \\
$\mathrm{Na}_{2} \mathrm{O}$ & $0.25-0.75$ \\
$\mathrm{~K}_{2} \mathrm{O}$ & $0.01-0.15$ \\
$\mathrm{P}_{2} \mathrm{O}_{5}$ & 0.00 \\
\hline
\end{tabular}



WATER

Table 6.5: Composition details of samples precipitated from geothermal water from the first series of work at Kawerau.

\begin{tabular}{|c|c|c|c|c|c|c|c|c|c|c|}
\hline $\begin{array}{l}\frac{0}{2} \\
\frac{\vec{\Xi}}{\pi} \\
\text { م. }\end{array}$ & $\begin{array}{c}\text { Total } \\
\% \\
\mathrm{SiO}_{2}\end{array}$ & $\begin{array}{c}\text { Total } \\
\% \\
\mathrm{CaO}\end{array}$ & $\begin{array}{c}\text { Total } \\
\% \\
\text { LOI a }\end{array}$ & $\begin{array}{c}\text { Total } \\
\mathrm{Ca} / \mathrm{Si} \text { b }\end{array}$ & $\% \mathrm{C}$ & $\begin{array}{c}\% \\
\mathrm{H}_{2} \mathrm{O}\end{array}$ & $\begin{array}{c}\text { Calc. } \\
\% \\
\mathrm{CaCO}_{3}\end{array}$ & $\begin{array}{c}\% \text { of } \\
\text { non- } \\
\mathrm{CaCO}_{3} \\
\mathrm{CaO}^{\mathrm{c}}\end{array}$ & $\begin{array}{c}\mathrm{Ca} / \mathrm{Si} \\
\text { without } \\
\mathrm{CaCO}_{3}\end{array}$ & $\begin{array}{c}\text { As } \\
(\mathrm{mg} / \mathrm{L})\end{array}$ \\
\hline K1 & 31.56 & 39.59 & 26.00 & 1.34 & 5.10 & 9.29 & 42.48 & 15.80 & 0.54 & 79.5 \\
\hline K2 & 21.00 & 49.44 & 27.17 & 2.52 & 5.83 & 8.63 & 48.59 & 22.23 & 1.13 & 141.1 \\
\hline K3 & 19.61 & 51.63 & 26.10 & 2.82 & 5.72 & 8.64 & 47.63 & 24.96 & 1.36 & 143.6 \\
\hline K4 & 16.34 & 55.09 & 26.30 & 3.61 & 5.93 & 9.43 & 49.41 & 27.42 & 1.80 & 104.8 \\
\hline K5 & 17.12 & 54.52 & 26.20 & 3.41 & 5.05 & 11.05 & 42.08 & 30.96 & 1.94 & 116.4 \\
\hline K6 & 15.67 & 55.64 & 26.44 & 3.80 & 4.57 & 12.83 & 38.10 & 34.30 & 2.35 & 131.7 \\
\hline K7 & 17.32 & 54.71 & 25.64 & 3.38 & 3.94 & 12.14 & 32.85 & 36.31 & 2.25 & 161.7 \\
\hline K8 & 16.42 & 55.59 & 25.80 & 3.63 & 6.05 & 8.33 & 50.40 & 27.37 & 1.79 & 167.8 \\
\hline K9 & 13.39 & 58.10 & 26.12 & 4.65 & 5.53 & 8.30 & 46.08 & 32.30 & 2.58 & 106.2 \\
\hline K10 & 12.99 & 58.16 & 26.87 & 4.80 & 5.95 & 8.06 & 49.59 & 30.39 & 2.51 & 116.9 \\
\hline K11 & 16.57 & 56.32 & 24.44 & 3.64 & 4.17 & 12.96 & 34.77 & 36.85 & 2.38 & 216.4 \\
\hline K12 & 15.51 & 57.55 & 24.76 & 3.98 & 4.65 & 10.81 & 38.74 & 35.85 & 2.48 & 197.8 \\
\hline K13 & 18.71 & 42.03 & 28.80 & 2.41 & 6.13 & 9.41 & 51.07 & 13.43 & 0.77 & 277.0 \\
\hline K14 & 19.17 & 52.38 & 25.27 & 2.93 & 4.97 & 9.42 & 41.45 & 29.17 & 1.63 & 222.9 \\
\hline K15 & 19.44 & 51.46 & 26.55 & 2.84 & 6.37 & 7.05 & 53.10 & 21.72 & 1.20 & 273.2 \\
\hline K16 & 15.96 & 56.00 & 25.67 & 3.76 & 5.43 & 9.25 & 45.27 & 30.65 & 2.06 & 205.4 \\
\hline K17 & 16.53 & 54.16 & 26.61 & 3.51 & 6.50 & 10.01 & 54.16 & 23.83 & 1.54 & 223.7 \\
\hline K18 & 15.29 & 56.32 & 25.57 & 3.95 & 4.19 & 11.65 & 34.92 & 36.77 & 2.58 & 209.2 \\
\hline K19 & 24.31 & 47.43 & 28.65 & 2.09 & 5.29 & 9.39 & 44.08 & 22.75 & 1.00 & 343.2 \\
\hline K20 & 18.55 & 52.92 & 25.77 & 3.06 & 5.31 & 9.68 & 44.28 & 28.13 & 1.62 & 220.2 \\
\hline K21 & 12.82 & 56.64 & 28.21 & 4.73 & 5.81 & 10.59 & 48.45 & 29.51 & 2.47 & 129.8 \\
\hline K22 & 18.37 & 52.82 & 26.46 & 3.08 & 5.95 & 9.53 & 49.60 & 25.04 & 1.46 & 241.0 \\
\hline K23 & 17.53 & 54.74 & 25.29 & 3.35 & 5.38 & 10.22 & 44.86 & 29.62 & 1.81 & 221.2 \\
\hline K24 & 16.44 & 56.03 & 25.06 & 3.65 & 4.06 & 16.09 & 33.79 & 37.11 & 2.42 & 222.3 \\
\hline K25 & 15.10 & 55.88 & 26.40 & 3.97 & 4.56 & 12.29 & 37.98 & 34.61 & 2.46 & 221.5 \\
\hline K26 & 15.81 & 54.23 & 26.82 & 3.68 & 4.08 & 12.61 & 33.98 & 35.20 & 2.39 & 217.3 \\
\hline K27 & 15.21 & 53.86 & 28.03 & 3.79 & 6.30 & 8.64 & 52.49 & 24.46 & 1.72 & 232.9 \\
\hline K28 & 21.72 & 47.66 & 27.99 & 2.35 & 5.79 & 7.71 & 48.23 & 20.65 & 1.02 & 313.9 \\
\hline K29 & 18.10 & 51.85 & 27.62 & 3.07 & 5.90 & 9.11 & 49.14 & 24.33 & 1.44 & 231.3 \\
\hline K30 & 20.93 & 49.11 & 27.64 & 2.51 & 5.30 & 11.33 & 44.17 & 24.38 & 1.25 & 265.4 \\
\hline K31 & 20.28 & 45.28 & 31.94 & 2.39 & 6.77 & 7.74 & 56.40 & 13.70 & 0.72 & 75.8 \\
\hline
\end{tabular}



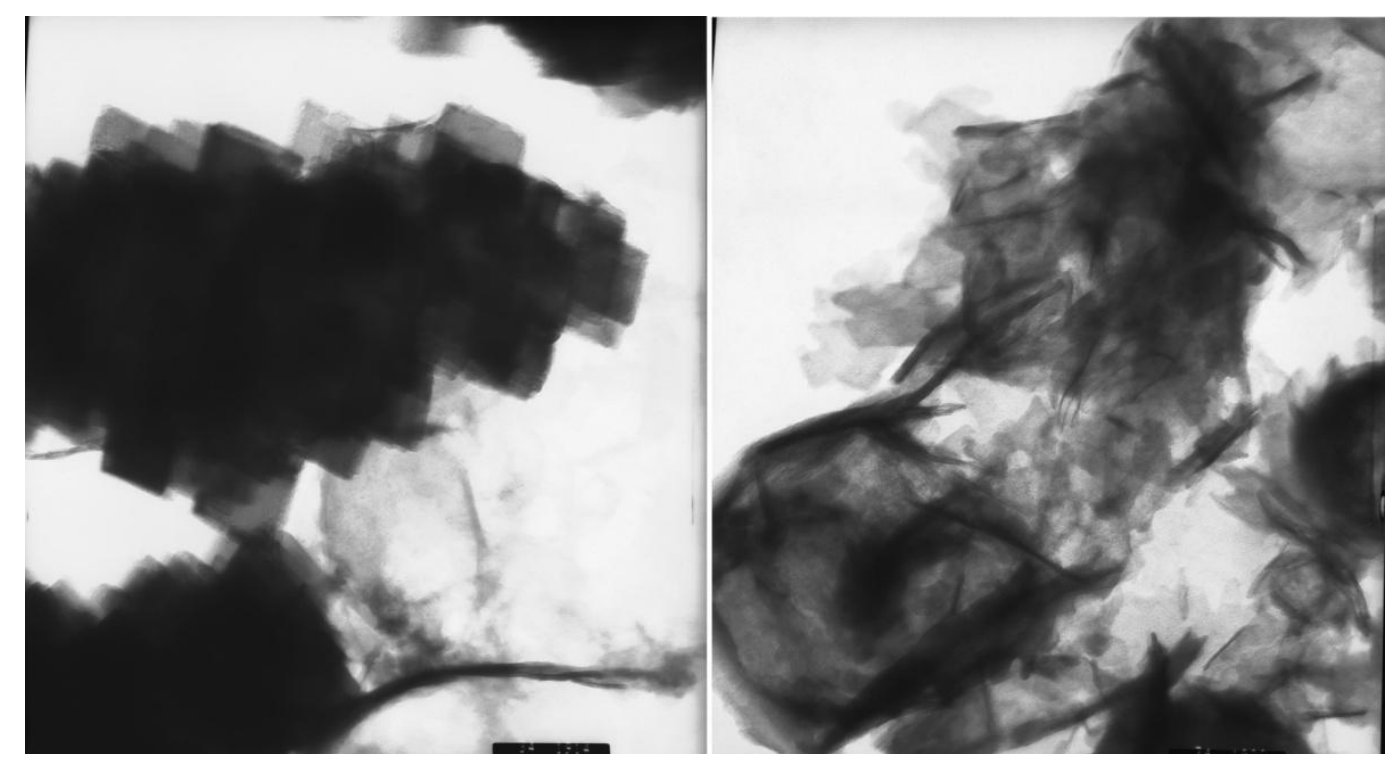

Figure 6.7: Transmission electron micrographs of K15 showing the presence of both calcium carbonate (left) and nano-structured calcium silicate (right) at a magnification of $105 \mathrm{~K}$.

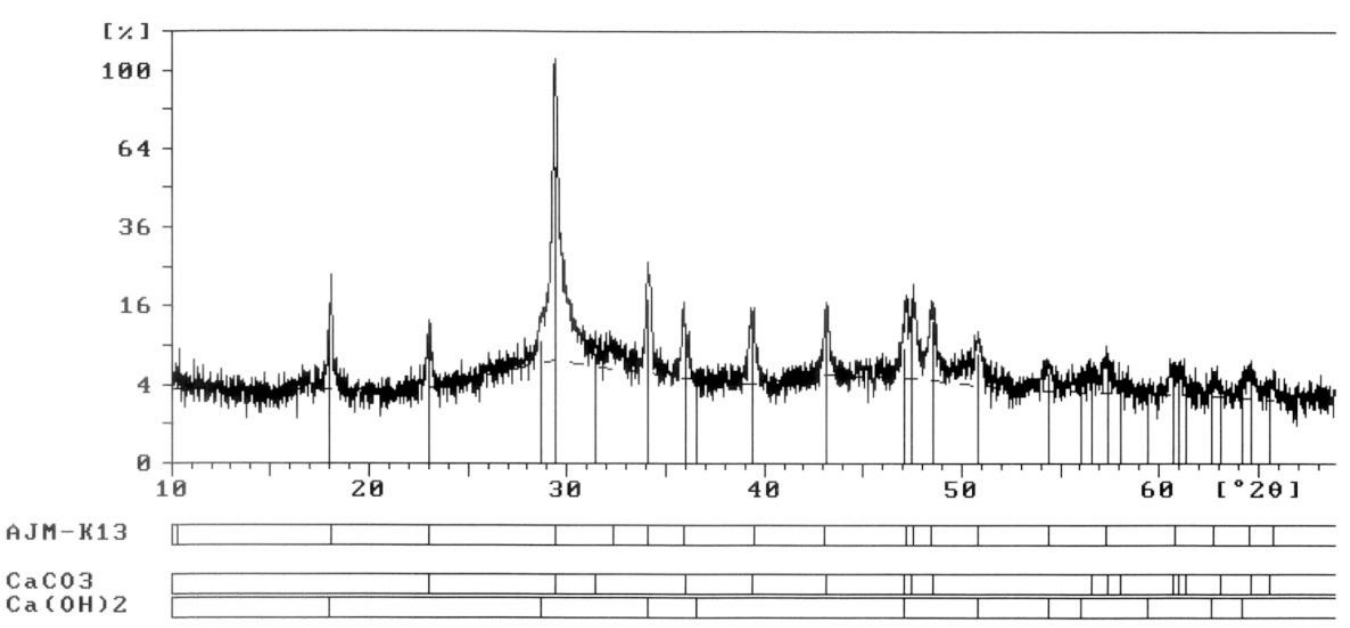

Figure 6.8: An X-ray diffractogram of K13 with sharp peaks due to the presence of calcium carbonate (PDF: 05-0586) and calcium hydroxide (PDF: 44-1481).

As mentioned by Harper (1994), one feature of the Kawerau discharge fluids is the low calcium and high bicarbonate concentrations. The fluid at boiling point becomes oversaturated with respect to calcium carbonate according to:

$$
\mathrm{Ca}^{2+}+2 \mathrm{HCO}_{3}^{-} \leftrightharpoons \mathrm{CaCO}_{3}+\mathrm{CO}_{2}(\mathrm{aq})+\mathrm{H}_{2} \mathrm{O}
$$

Upon boiling, the resulting carbon dioxide loss drives the equilibrium to the right, therefore causing the precipitation of calcium carbonate. The increase in $\mathrm{pH}$ upon boiling also increases the ratio of $\mathrm{HCO}_{3}^{-} / \mathrm{CO}_{3}^{2-}$ therefore further driving the equilibrium towards calcium carbonate formation as well as the 
addition of further calcium ions. The calculated proportion of calcium carbonate in the samples varied from a third to over a half of the samples overall mass (Figure 6.9).

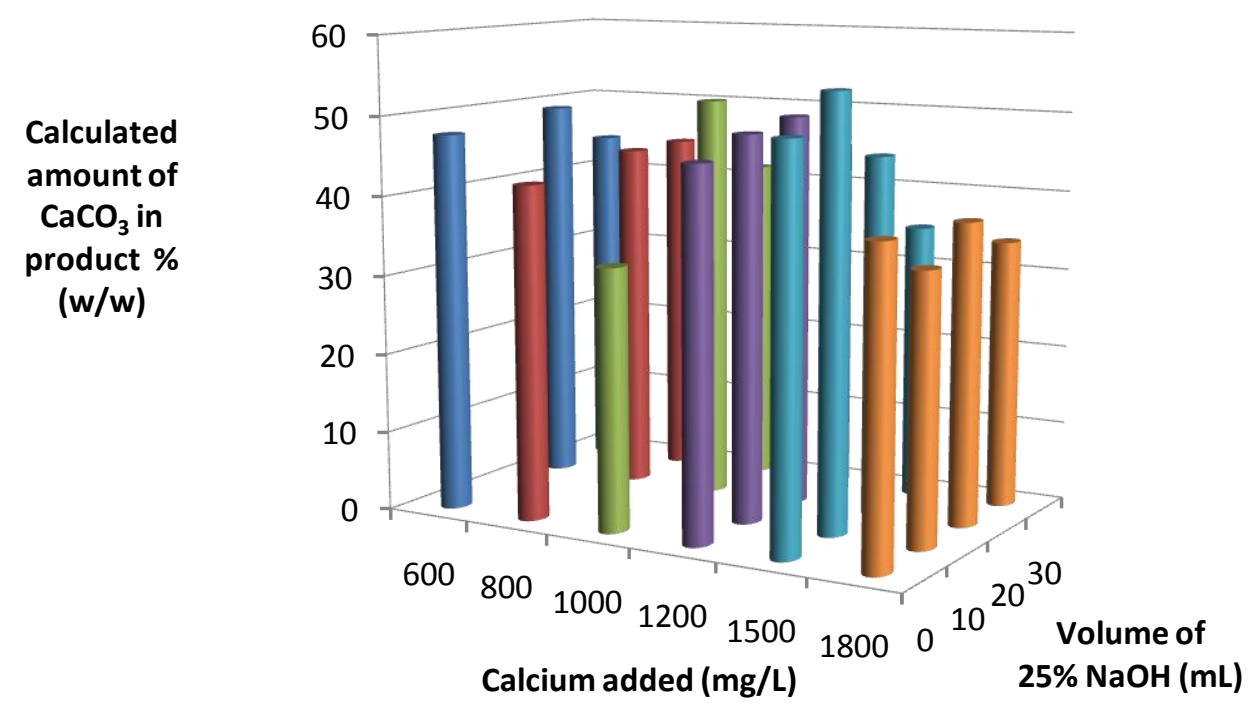

Figure 6.9: Calculated amount of calcium carbonate present in materials precipitated from geothermal water at Kawerau.

The thermal analysis curve of sample K19 is greatly influenced by the large amount of calcium carbonate present (Figure 6.10). A typical nano-structured calcium silicate that had been previously dried at $110{ }^{\circ} \mathrm{C}$ will lose ca. $22 \%$ of its weight when heated up to $800{ }^{\circ} \mathrm{C}$ due to the loss of chemically bound water and the condensation of surface silanol groups. However, pure calcite decomposes at above $600{ }^{\circ} \mathrm{C}$ (Earnest, 1988), with a $44 \%$ weight loss through the volatilisation of carbon dioxide. The decrease in mass observed for K19 can be calculated from the calcium carbonate and calcium silicate components separately. Based upon the sample's calculated calcium carbonate content of $44 \%$ from Leco analysis (Table 6.5), a ca. 19\% weight loss would be expected from the decomposition of calcium carbonate present (ie. $44 \%$ of carbon dioxide in the $44 \%$ calcium carbonate component). A ca. $12 \%$ weight loss due to the removal of water from the nano-structured calcium silicate would also be expected (ie. the $22 \%$ water from the $54 \%$ calcium silicate present). The combined weight loss of $31 \%$ from each of the components is close to the $35 \%$ weight loss observed in Figure 6.10. 


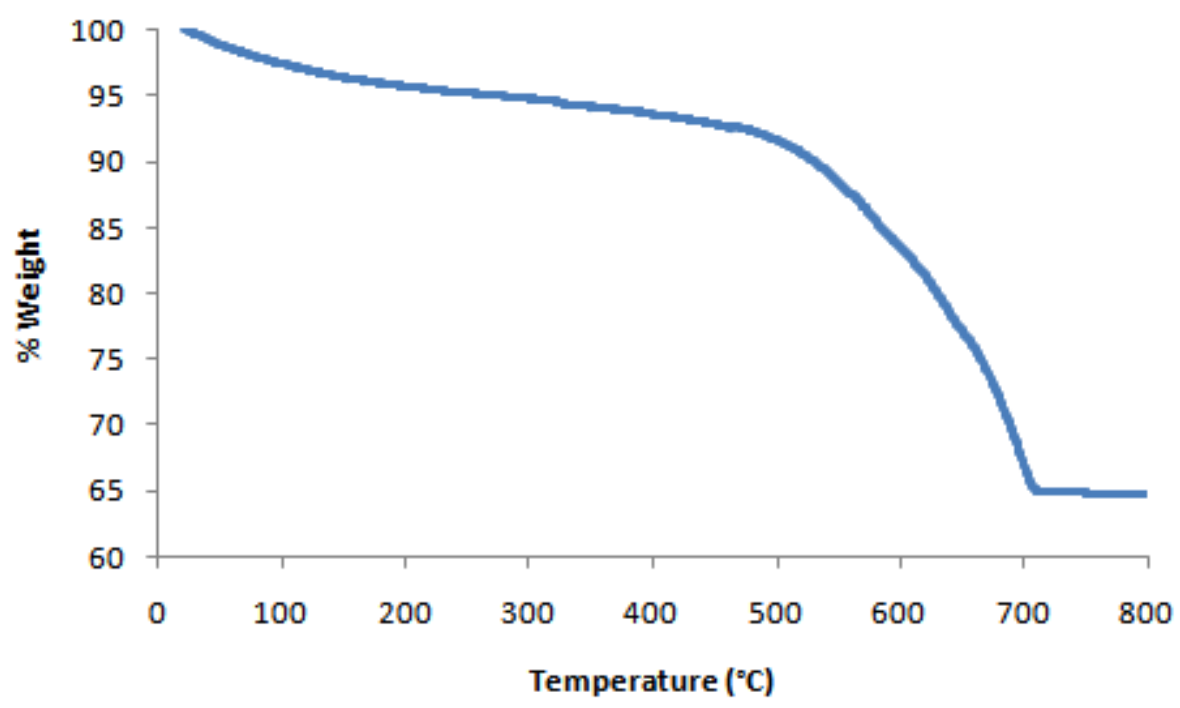

Figure 6.10: Thermogravimetric analysis of sample K19.

Given the surprisingly large amount of calcium carbonate present, the oil absorption capacities of the mixed materials were reasonably large, especially as the samples had not been treated in any way to prevent collapse upon drying (Figure 6.11). The oil absorption capacity of some of the materials exceeded some commercially available precipitated silicas with sample K19 measured to have the largest oil absorption of $250 \mathrm{~g} / 100 \mathrm{~g}$.

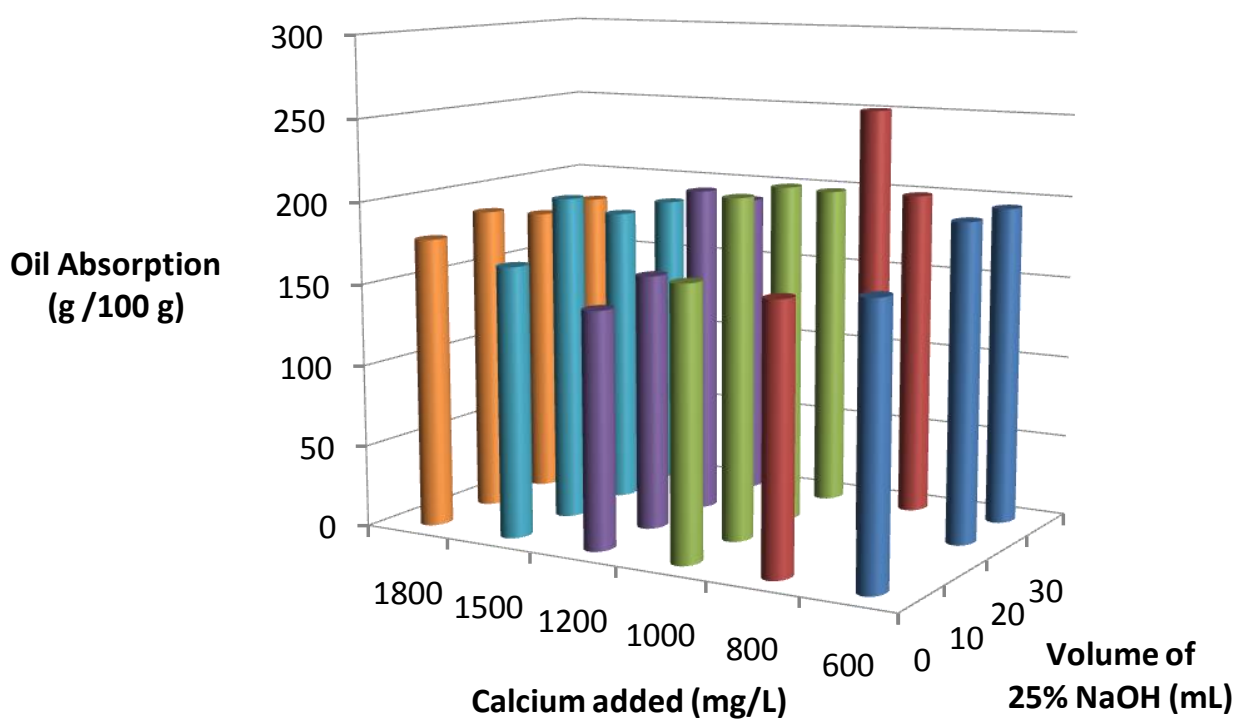

Figure 6.11: Oil absorption capacities of materials precipitated from geothermal water at Kawerau 
Sample K19 had a calculated $\mathrm{Ca} / \mathrm{Si}$ ratio of 1 (when excluding calcium associated with calcium carbonate), as found in optimised materials prepared in the laboratory. The distinctive porous nano-structure was evident in this material when studied with a scanning electron microscope (Figure 6.12).

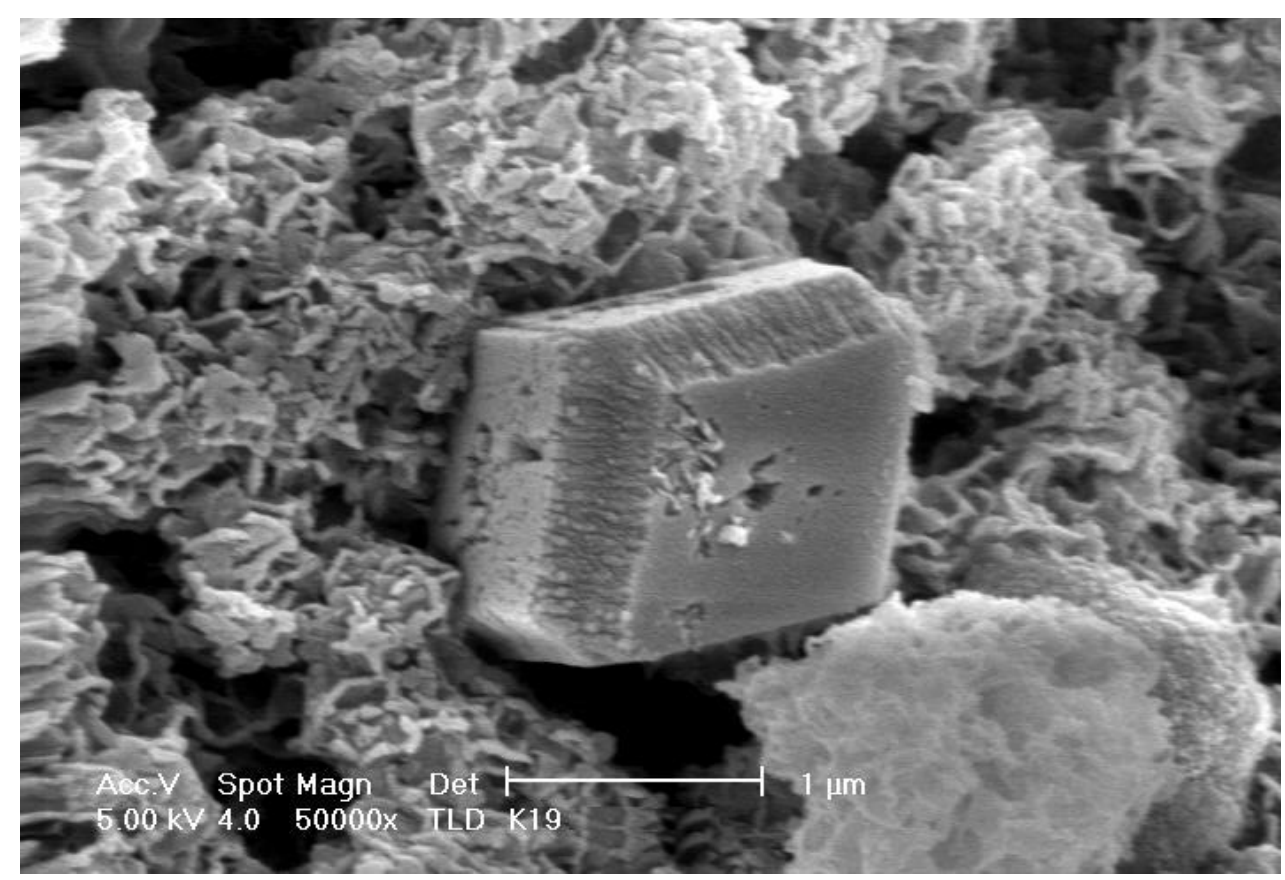

Figure 6.12: A scanning electron micrograph of K19 that shows a calcium carbonate crystal with relatively little porosity, amongst the typical porous structure of nano-structured calcium silicate.

The concentration of monomeric silica remaining in solution following the precipitation of K19 was only $68 \mathrm{mg} / \mathrm{L}$, which is well below the solubility of amorphous silica at room temperature for a pH above 10 (and also at lower pHs). The potential for the polymerisation of the silica remaining in solution, even if all the thermal energy of the water is used, would therefore be minimal.

As discussed in Chapter 4, one method of reinforcing silica is by heat-ageing a wet gel as this causes a redistribution of silica in the structure. The areas of large positive curvature that have a higher solubility are dissolved and the released monomeric silica deposits upon areas of narrow negative curvature that have lower solubility. It may be possible that if a large proportion of the calcium hydroxide added reacts to form calcium carbonate, the resulting excess monomeric silica in the geothermal water could reinforce the nano-structured calcium silicate that is formed and therefore strengthen the structure to 
prevent collapse. However, the calculated Ca/Si ratio of 1 for K19 once the calcium content due to the calcium carbonate is excluded, does not suggest that this has occurred as a reinforced sample would be expected to be more silicon rich.

As a result of the varying calcium carbonate content of the samples, it is difficult to see any trends in the oil absorption capacity produced under the differing conditions. The oil absorption capacity of K19 significantly exceeds even those samples produced with a slightly different $\mathrm{pH}$ or calcium concentration, with oil absorption capacities approximately 20\% less of ca. 200 g/100 g measured. One apparent trend is that the oil absorption capacities of samples precipitated without the addition of sodium hydroxide were less than those precipitated in more alkaline conditions. This indicates the importance of $\mathrm{pH}$, which was later also found with experiments using sodium silicate. The surface areas of the samples are also greatly reduced than expected from pure nano-structured calcium silicate, with no obvious relationships between the reaction variables and the resulting surface areas (Figure 6.13).

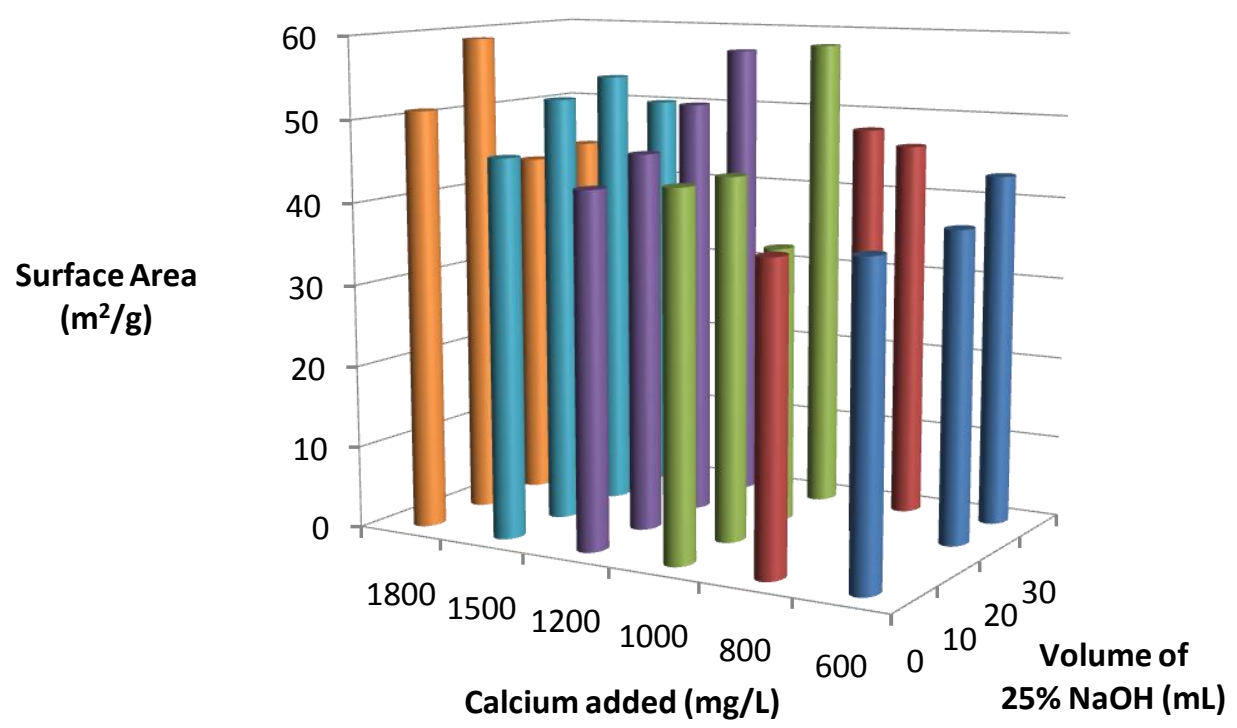

Figure 6.13: Surface areas of materials precipitated from geothermal water at Kawerau.

One concern for the use of geothermal water as the source of monomeric silica for the precipitation of nano-structured calcium silicate is the arsenic content of the resulting materials due to the toxicity of arsenic compounds. Arsenic is 
commonly found in the geothermal waters of the Taupo Volcanic Zone as it is found in the greywacke, schists, and tertiary volcanics that occur in the region (Ellis \& Mahon, 1977). The addition of calcium hydroxide may result in the precipitation of calcium arsenate, or the arsenate anion could potentially bind to the accessible surface calcium groups of the nano-structured silicate. If nanostructured calcium silicate precipitated from geothermal water is to be used in paper as a filler or in a coating formulation, the arsenic content would need to be within health regulations. The arsenic content of all samples was therefore determined by XRF (Table 6.5 and Figure 6.14).

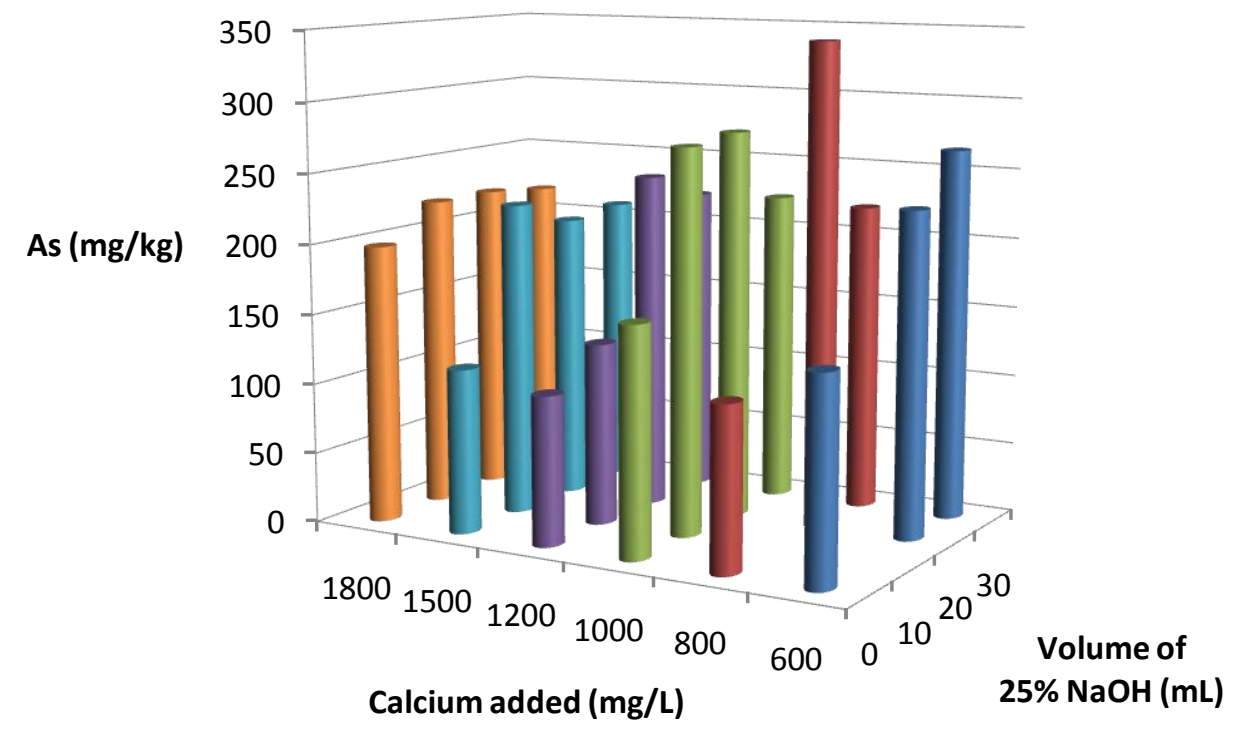

Figure 6.14: Arsenic content of materials precipitated using geothermal water at Kawerau.

The World Health Organisation reports that the smallest, fatal, single dose for a human is in the range of 70 to $180 \mathrm{mg}$. Based upon the calcium silicate with the highest arsenic content (K19 with $343 \mathrm{mg} / \mathrm{kg}$ ), this would require the consumption of 204 to $525 \mathrm{~g}$ of the pure silicate or 4.1 to $10.5 \mathrm{~kg}$ of paper filled with a $5 \%$ loading. With long-term exposure, significant toxic effects can be expected to occur above a daily oral intake of 100 to $200 \mu \mathrm{g}$ which would require 0.29 to $0.58 \mathrm{~g}$ of pure calcium silicate or 5.8 to $11.7 \mathrm{~g}$ of paper filled with a $5 \%$ loading to be consumed. Ministry of Health guidelines (Durham, 1997) for graphic materials (which includes materials designed to leave a trace, natural and synthetic textiles, and paper and board materials), are based upon 
leachability, bioavailability, and exposure to other sources of toxic elements in the average diet. A reasonable limit for the ingestion of $8 \mathrm{mg} /$ day of graphic material under likely conditions of misuse (such as chewing or sucking) is used. The maximum limit set for the leachable content of arsenic is $25 \mathrm{mg} / \mathrm{kg}$ of graphic material. This is greater than that present in a filled paper with a $5 \%$ loading of $17.5 \mathrm{mg} / \mathrm{kg}$ for the material with the highest arsenic concentration. The loading of nano-structured calcium silicate when used in a coating formulation is likely to be less than $5 \%$ and would therefore be even further beneath the health guidelines.

When the arsenic content is plotted against the oil absorption capacity (Figure 6.15), there seems to be a relationship between the two, although the reason for this is unclear. It could indicate that the arsenic species is binding to the calcium silicate with a more accessible surface after the material is formed. The other possibility is that the arsenic may in some way be aiding in the development of a highly porous structure.

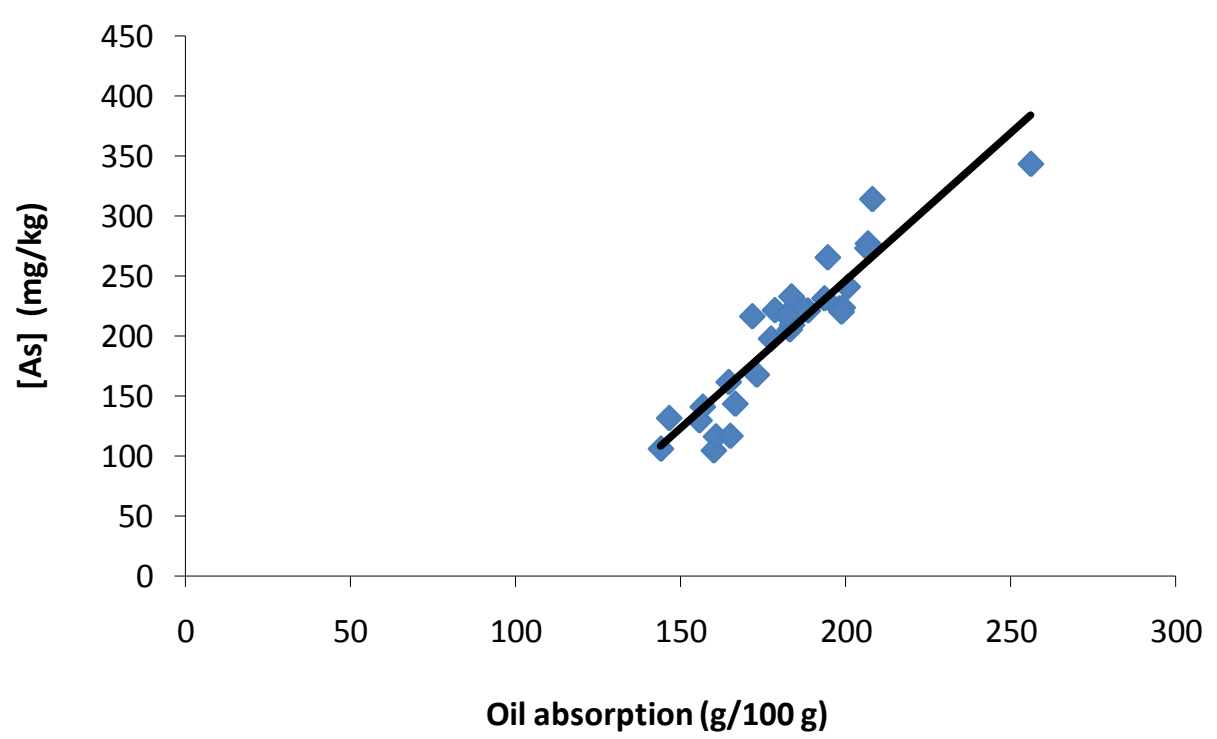

Figure 6.15: Relationship between the oil absorption capacities of materials precipitated using geothermal water at Kawerau and the arsenic content.

As such, further analysis of calcium silicates prepared using geothermal water is necessary, especially if the process is to be optimised to produce nanostructured calcium silicates with larger oil absorption capacities. Due to the 
variable nature of the composition of geothermal water and as a result of how changeably the field can be operated, constant monitoring of the arsenic content in the material produced using the water would be necessary if the material was to be manufactured on a commercial scale.

Two reactions to form precipitated silica (K1 and K31) were also performed whereby the geothermal water was set aside for an hour so that polymerisation would occur before the addition of the calcium hydroxide as the coagulant (Table 6.6). The change in monomeric silica concentration during the polymerisation reaction was monitored by taking samples of the solution throughout the process.

Table 6.6: Details of reactions to form precipitated silica using geothermal water during the first series of work at Kawerau

\begin{tabular}{|c|c|c|c|c|c|c|c|c|}
\hline $\begin{array}{c}\text { Sample } \\
\text { name }\end{array}$ & $\begin{array}{c}\text { Calcium } \\
\text { added } \\
(\mathrm{mg} / \mathrm{L})\end{array}$ & $\begin{array}{l}\text { Time } \\
(\mathrm{min})\end{array}$ & $\mathrm{pH}$ & $\begin{array}{c}\text { Temp. } \\
\left({ }^{\circ} \mathrm{C}\right)\end{array}$ & $\begin{array}{l}{\left[\mathrm{SiO}_{2}\right]_{\mathrm{m}}} \\
(\mathrm{mg} / \mathrm{L})\end{array}$ & $\begin{array}{c}\text { Product } \\
\text { weight } \\
\text { (g) }\end{array}$ & $\begin{array}{c}\text { Oil } \\
\text { absorption } \\
\text { (g/100g) }\end{array}$ & $\begin{array}{c}\text { Surface } \\
\text { area } \\
\left(\mathrm{m}^{2} / \mathrm{g}\right)\end{array}$ \\
\hline \multirow[t]{9}{*}{ K1 } & 500 & 0 & 7.57 & 95.7 & 495 & & & \\
\hline & & 1 & 7.62 & 93.6 & 507 & & & \\
\hline & & 2.5 & 7.64 & 92.9 & 524 & & & \\
\hline & & 5 & 7.69 & 91.8 & 505 & & & \\
\hline & & 10 & 7.74 & 88.3 & 478 & & & \\
\hline & & 20 & 7.77 & 80.2 & 462 & & & \\
\hline & & 30 & 7.83 & 78.1 & 462 & & & \\
\hline & & 45 & 7.93 & 73.4 & 429 & & & \\
\hline & & 60 & 7.93 & 69.8 & & 13.88 & 155 & 116 \\
\hline \multirow[t]{7}{*}{ K31 } & 700 & 0.5 & 7.95 & 95.7 & 500 & & & \\
\hline & & 1 & 7.84 & 95.9 & 115 & & & \\
\hline & & 2 & 7.85 & 94.7 & 128 & & & \\
\hline & & 5 & 7.82 & 92.4 & 114 & & & \\
\hline & & 10 & 7.90 & 88.7 & 484 & & & \\
\hline & & 20 & 8.05 & 81.6 & 429 & & & \\
\hline & & 30 & 7.94 & 75.5 & 456 & 9.72 & 135 & 48 \\
\hline
\end{tabular}

Unfortunately the polymerisation of some samples continued after being collected from the slurry of K31, but a polymerisation curve was able to be plotted from data collected from the K1 reaction (Figure 6.16). The initial monomeric silica concentration is less that expected for both of these samples and may indicate that some degree of polymerisation has already occurred. The decrease in monomeric silica concentration over time for $\mathrm{K} 1$ is also likely to be 
a result of the solution cooling and therefore decreasing the solubility that causes further polymerisation. Following polymerisation, a relatively low concentration of calcium was added to coagulate the silica sol particles that have formed. As less calcium was added to form the precipitated silica, the $\mathrm{Ca} / \mathrm{Si}$ ratio once the calcium from calcium carbonate is excluded, is lower than for the nano-structured calcium silicates prepared. The effect of increasing the calcium concentration for coagulation in K31 caused an increase in the amount of calcium carbonate formed and therefore both the surface area and oil absorption capacity of the material formed decreased (Table 6.6).

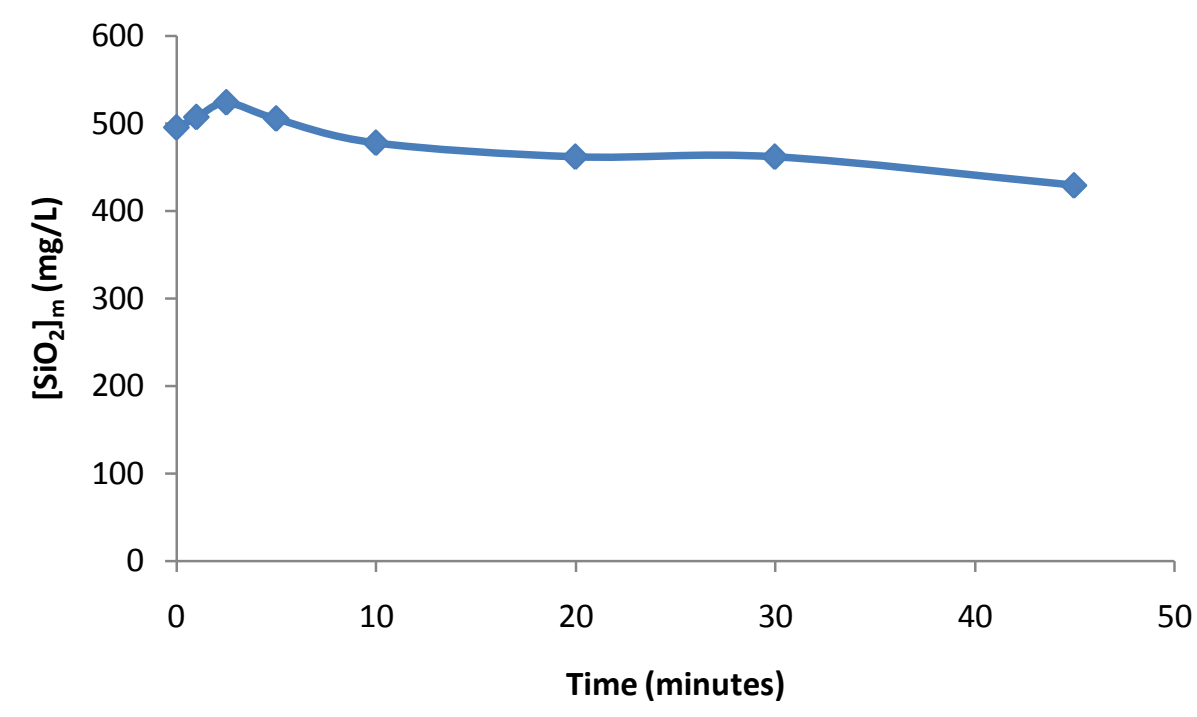

Figure 6.16: The polymerisation curve for the formation of precipitated silica using geothermal water from the Kawerau field (sample K1).

A small group of experiments were also performed at the Rotokawa geothermal power station in the Taupo Volcanic Zone (Table 6.7). The Rotokawa geothermal field is located about $14 \mathrm{~km}$ northeast of Taupo and the water is used for power generation at a binary plant. The temperature of the resource underground reaches up to $320^{\circ} \mathrm{C}$. The geothermal water was obtained from a pipeline that was under pressure (where samples to analyse the water were usually taken) and was therefore run through a cooling coil to prevent the fluid from flashing. The remaining methodology followed that used for reactions using geothermal water from the Kawerau field. 
CHAPTER 6: NANO-STRUCTURED CALCIUM SILICATE FROM GEOTHERMAL WATER

Table 6.7: Details of reactions using geothermal water from the Rotokawa field

\begin{tabular}{|c|c|c|c|c|c|c|c|c|}
\hline 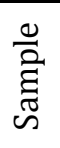 & $\begin{array}{c}\text { Calcium } \\
\text { added } \\
(\mathrm{mg} / \mathrm{L})\end{array}$ & $\begin{array}{l}\text { Time } \\
\text { (min) }\end{array}$ & $\mathrm{pH}$ & $\begin{array}{c}\text { Temp. } \\
\left({ }^{\circ} \mathrm{C}\right)\end{array}$ & $\begin{array}{l}{\left[\mathrm{SiO}_{2}\right]_{\mathrm{m}}} \\
(\mathrm{mg} / \mathrm{L})\end{array}$ & $\begin{array}{l}\text { Product } \\
\text { weight } \\
\text { (g) }\end{array}$ & $\begin{array}{c}\text { Oil } \\
\text { absorption } \\
(\mathrm{g} / 100 \mathrm{~g})\end{array}$ & $\begin{array}{c}\text { Surface } \\
\text { area } \\
\left(\mathrm{m}^{2} / \mathrm{g}\right)\end{array}$ \\
\hline \multirow[t]{9}{*}{$\mathrm{R} 1$} & 500 & 0 & & & 496 & & & \\
\hline & & 0.5 & & & 512 & & & \\
\hline & & 1 & 6.60 & & 477 & & & \\
\hline & & 2 & 6.56 & 69.7 & 487 & & & \\
\hline & & 5 & 6.66 & 68.4 & 476 & & & \\
\hline & & 10 & & & 461 & & & \\
\hline & & 18 & 6.87 & 61.2 & & & & \\
\hline & & 20 & & & 459 & & & \\
\hline & & 30 & 6.96 & 55.8 & 458 & 8.61 & 149 & 37.7 \\
\hline \multirow[t]{4}{*}{ R2 } & 1500 & 0 & 6.48 & 64.9 & & & & \\
\hline & & 2 & 10.6 & 57.0 & 26 & & & \\
\hline & & 5 & 11.14 & 60.0 & 18 & & & \\
\hline & & 15 & & & 18 & 27.11 & 166 & 44.2 \\
\hline \multirow[t]{3}{*}{ R3 } & 1200 & 0 & 6.53 & 69.0 & & & & \\
\hline & & 3.5 & 10.14 & 67.7 & & & & \\
\hline & & 15 & 10.41 & 62.0 & 200 & 23.66 & 148 & 44.3 \\
\hline \multirow[t]{5}{*}{$\mathrm{R} 4$} & 1000 & 0 & 6.59 & 68.0 & & & & \\
\hline & & 0.33 & 10.00 & 66.8 & & & & \\
\hline & & 2 & 10.14 & 65.7 & & & & \\
\hline & & 5 & 10.21 & 63.4 & 219 & & & \\
\hline & & 15 & 10.30 & 58.4 & 198 & 18.94 & 144 & 40.8 \\
\hline \multirow[t]{5}{*}{ R5 } & 1000 & 0 & 6.48 & 63.2 & & & & \\
\hline & & 1 & 10.85 & 61.1 & & & & \\
\hline & & 3 & 10.99 & 60.8 & & & & \\
\hline & & 5 & 11.14 & 58.2 & 352 & & & \\
\hline & & 15 & 11.13 & 55.3 & 313 & 21.02 & 142 & 46.5 \\
\hline \multirow[t]{4}{*}{ R6 } & 2000 & 0.33 & 10.51 & 61.2 & & & & \\
\hline & & 3 & 11.02 & 66.7 & & & & \\
\hline & & 5 & 11.21 & 65.1 & 13 & & & \\
\hline & & 15 & 11.39 & 59.1 & 10 & 34.55 & 160 & 50.5 \\
\hline \multirow[t]{3}{*}{ R7 } & 2000 & 0.67 & 10.68 & 62.9 & & & & \\
\hline & & 5 & 11.3 & 57.6 & 16 & & & \\
\hline & & 15 & 11.73 & 46.3 & 14 & 8.39 & 155 & 40.5 \\
\hline
\end{tabular}

As few reactions were undertaken, no sodium hydroxide was added to increase the $\mathrm{pH}$, although the $\mathrm{pH}$ was lower than that from the Kawerau resource because the water had not been flashed. Like the materials prepared from Kawerau geothermal water, the materials precipitated at the Rotokawa field also contained a large proportion of calcium carbonate as observed with a transmission electron microscope. This presence of calcium carbonate is also suggested by the high $\mathrm{Ca} / \mathrm{Si}$ ratios of the samples (Table 6.8) as determined by 
$\mathrm{X}$-ray fluorescence spectroscopy. The very low surface areas of the samples prepared also indicate that a large proportion of calcium carbonate is also present. A summary of the minor components present of all samples prepared using geothermal water at Rotokawa given in (Table 6.9).

Table 6.8: Composition of materials formed using Rotokawa geothermal water as determined using XRF

\begin{tabular}{ccccc}
\hline Sample & ${\text { \% } \mathrm{SiO}_{2}}$ & \%CaO & \%LOI & $\mathrm{Ca} / \mathrm{Si}$ \\
\hline $\mathrm{R} 1$ & 26.16 & 48.07 & 23.58 & 1.97 \\
$\mathrm{R} 2$ & 22.58 & 51.97 & 23.14 & 2.47 \\
$\mathrm{R} 3$ & 18.75 & 55.06 & 23.96 & 3.15 \\
$\mathrm{R} 4$ & 18.73 & 54.70 & 24.03 & 3.13 \\
$\mathrm{R} 5$ & 17.25 & 56.45 & 24.05 & 3.51 \\
$\mathrm{R} 6$ & 18.36 & 55.64 & 23.54 & 3.25 \\
R7 & 21.96 & 52.05 & 23.49 & 2.54 \\
\hline
\end{tabular}

Table 6.9: The proportion of minor components present for all samples prepared using geothermal water from the Rotokawa field as determined by XRF spectroscopy.

\begin{tabular}{cc}
\hline Component & \% range \\
\hline $\mathrm{TiO}_{2}$ & $0.04-0.06$ \\
$\mathrm{Al}_{2} \mathrm{O}_{3}$ & $0.13-0.20$ \\
$\mathrm{Fe}_{2} \mathrm{O}_{3}$ & $0.05-0.06$ \\
$\mathrm{MnO}$ & $0.00-0.01$ \\
$\mathrm{MgO}$ & $1.13-1.31$ \\
$\mathrm{Na}_{2} \mathrm{O}$ & $0.29-0.41$ \\
$\mathrm{~K}_{2} \mathrm{O}$ & $0.03-0.06$ \\
$\mathrm{P}_{2} \mathrm{O}_{5}$ & 0.00 \\
\hline
\end{tabular}

A reaction was undertaken to form a precipitated silica (R1), although due to time constraints only 30 minutes was allowed for polymerisation (unlike the Kawerau reactions $\mathrm{K} 1$ and $\mathrm{K} 31$ that took place over 60 minutes). The polymerisation curve was determined by sampling the solution during the reaction, and like that from the Kawerau water showed only a slight decrease in monomeric silica concentration probably as a result of the decreasing solubility as the fluid cooled (Figure 6.17). This suggests that either the concentration of total silica available is at the solubility for the fluid conditions, or that some degree of polymerisation had already occurred. The manager at the site 
mentioned that the most recent analysis of the fluid by the Institute of Geological and Nuclear Sciences had measured a monomeric silica concentration of $670 \mathrm{mg} / \mathrm{L}$ from the same outlet. This suggests that either some polymerisation had already occurred or that the field conditions had changed.

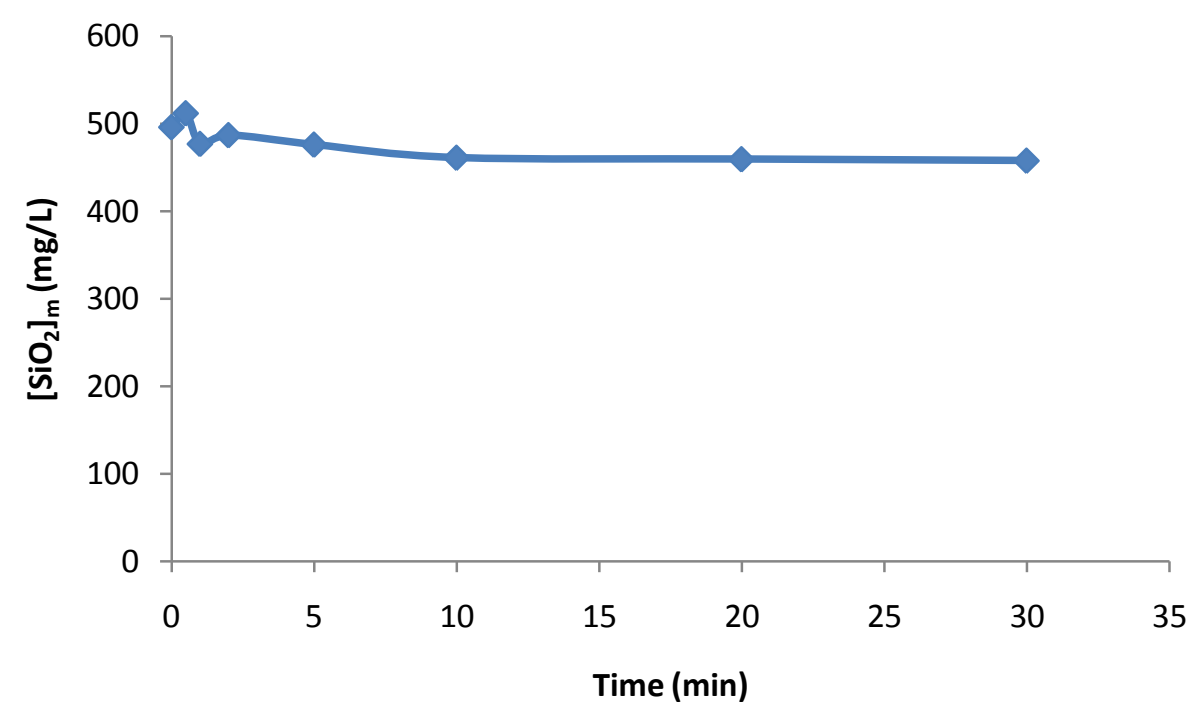

Figure 6.17: Polymerisation curve for the formation of precipitated silica using geothermal water from the Rotokawa field.

The optical properties of all samples prepared during this first section of work at both the Kawerau and Rotokawa geothermal fields are given in Appendix B.

\subsection{The second series of reactions using geothermal water}

Later in the research programme, the opportunity arose to carry out further experiments at the Kawerau geothermal field. The intention of this series of experiments was to gather more information surrounding the reaction that produced the highest oil absorption capacity from the previous series of reactions (K19). To this end, the concentration of calcium to be added was limited to the range of 600 to $1200 \mathrm{mg} / \mathrm{L}$ and the volume of $25 \%(\mathrm{w} / \mathrm{w})$ of sodium hydroxide to be added limited from 10 to $25 \mathrm{~mL}$. This series of experiments also followed the development of 2-ethoxyethanol for preventing the structure from collapsing when removing water from the pores during 
drying. Consequently each slurry produced was divided in the laboratory, with half of the slurry dried from water as per the earlier series, and the other half treated with 2-ethoxyethanol. The methods used for the preparation of the materials followed those of the previous series as closely as possible with the details of each given in Table 6.10.

Unfortunately, the oil absorption capacities of water washed samples from this series of experiments were very different to those achieved from the previous series (Figure 6.18). While previously several samples had oil absorptions in excess of $200 \mathrm{~g} / 100 \mathrm{~g}$, materials from this series of experiments all had oil absorption capacities below 150 g/ 100 g. Significant variations in the chemical composition of production wells can occur over time due to mass flow changes in the feed zones to the wells due to operational conditions.

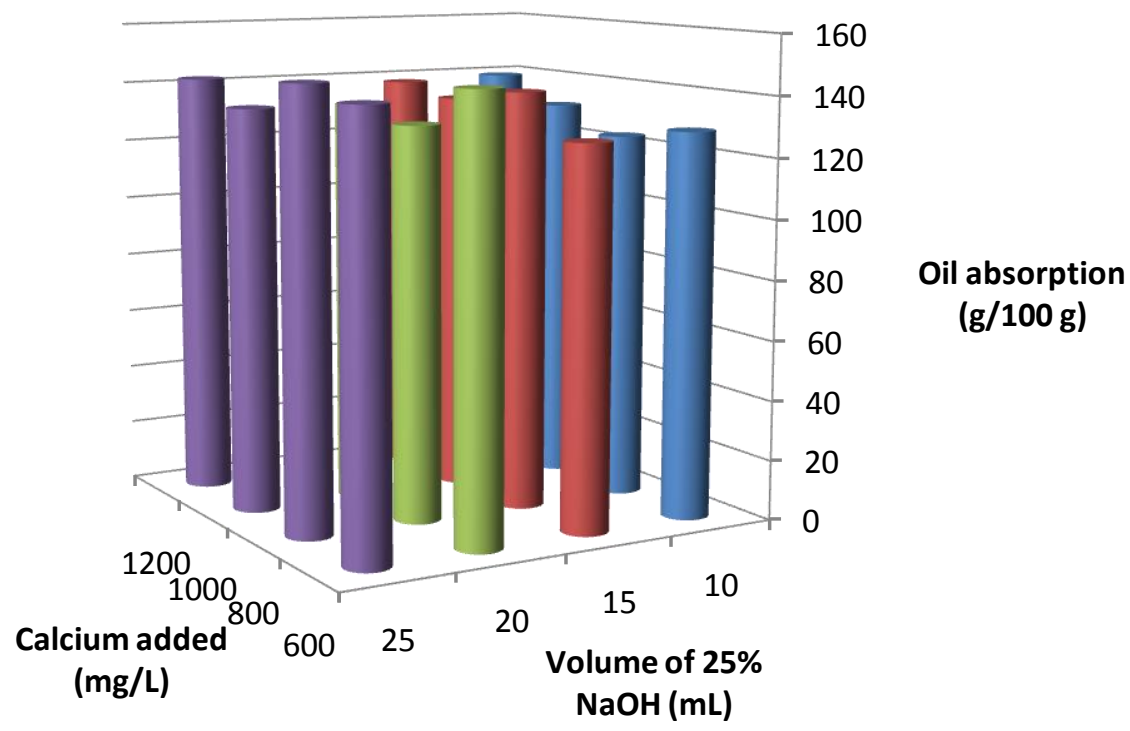

Figure 6.18: Oil absorption capacities of water washed materials precipitated from geothermal water at Kawerau from second series of experiments.

The effect of trace elements upon the development of the structure is also unknown. These elements, that could have been in different concentrations for the two different trials, could be incorporated into the structure and therefore alter the development of materials porosity. As a period of three years had passed between both series of experiments, it is possible that this variation of the geothermal water is the reason for the difference in properties observed between the two series. 
CHAPTER 6: NANO-STRUCTURED CALCIUM SILICATE FROM GEOTHERMAL WATER

Table 6.10: Details of reaction conditions for the precipitation of materials from geothermal water during the second series of work at Kawerau.

\begin{tabular}{|c|c|c|c|c|c|c|c|}
\hline \multirow{2}{*}{$\begin{array}{c}\text { Calcium } \\
\text { added } \\
(\mathrm{mg} / \mathrm{L})\end{array}$} & \multirow{2}{*}{$\begin{array}{c}\text { Vol. } \\
\mathrm{NaOH} \\
(\mathrm{mL})\end{array}$} & \multirow{2}{*}{$\begin{array}{l}\text { Time } \\
\text { (min) }\end{array}$} & \multirow[t]{2}{*}{$\mathrm{pH}$} & \multirow{2}{*}{$\begin{array}{l}\text { Temp } \\
\left({ }^{\circ} \mathrm{C}\right)\end{array}$} & \multirow{2}{*}{$\begin{array}{l}{\left[\mathrm{SiO}_{2}\right]_{\mathrm{m}}} \\
(\mathrm{mg} / \mathrm{L})\end{array}$} & \multicolumn{2}{|c|}{ Oil absorption (g/100 g) } \\
\hline & & & & & & $\begin{array}{c}\text { Water } \\
\text { washed }\end{array}$ & 2EE washed \\
\hline \multirow[t]{4}{*}{600} & 10 & 1 & 9.8 & 91.3 & & & \\
\hline & & 5 & 10.0 & 84.6 & & & \\
\hline & & 15 & 10.2 & 73.2 & 366 & & \\
\hline & & 48 & 10.4 & 63.5 & & 129 & 142 \\
\hline \multirow[t]{4}{*}{600} & 15 & 1 & 10.0 & 91.1 & & & \\
\hline & & 5 & 10.2 & 84.0 & & & \\
\hline & & 16 & 10.5 & 71.7 & 108 & & \\
\hline & & 55 & 10.7 & 60.3 & & 127 & 118 \\
\hline \multirow[t]{4}{*}{600} & 20 & 1 & 10.2 & 92.5 & & & \\
\hline & & 5 & 10.4 & 83.6 & & & \\
\hline & & 15 & 10.7 & 72.5 & 125 & & \\
\hline & & 42 & 11.0 & 64.2 & & 146 & 249 \\
\hline \multirow[t]{4}{*}{600} & 25 & 1 & 10.3 & 92.0 & & & \\
\hline & & 5 & 10.5 & 84.5 & & & \\
\hline & & 15 & 10.9 & 71.8 & 234 & & \\
\hline & & 41 & 11.1 & 64.2 & & 143 & 213 \\
\hline \multirow[t]{4}{*}{800} & 10 & 1 & 9.9 & 92.4 & & & \\
\hline & & 5 & 10.2 & 82.4 & & & \\
\hline & & 15 & 10.5 & 67.8 & 105 & & \\
\hline & & 60 & 10.8 & 56.3 & & 124 & 128 \\
\hline \multirow[t]{4}{*}{800} & 15 & 1 & 10.0 & 91.8 & & & \\
\hline & & 5 & 10.3 & 83.4 & & & \\
\hline & & 14.5 & 10.6 & 70.3 & 109 & & \\
\hline & & 44 & 10.8 & 61.6 & & 140 & 169 \\
\hline \multirow[t]{4}{*}{800} & 20 & 1 & 10.2 & 92.0 & & & \\
\hline & & 5 & 10.4 & 84.3 & & & \\
\hline & & 15 & 10.8 & 71.8 & 116 & & \\
\hline & & 49 & 11.0 & 61.4 & & 131 & 222 \\
\hline \multirow[t]{4}{*}{800} & 25 & 1 & 10.3 & 91.8 & & & \\
\hline & & 5 & 10.6 & 83.6 & & & \\
\hline & & 14.5 & 11.0 & 71.0 & 136 & & \\
\hline & & 45 & 11.2 & 62.3 & & 146 & 218 \\
\hline \multirow[t]{4}{*}{1000} & 10 & 1 & 9.9 & 92.2 & & & \\
\hline & & 5 & 10.2 & 83.5 & & & \\
\hline & & 13 & 10.5 & 72.8 & 328 & & \\
\hline & & 43 & 10.7 & 62.2 & & 131 & 139 \\
\hline \multirow[t]{4}{*}{1000} & 15 & 1 & 10.2 & 91.7 & & & \\
\hline & & 5 & 10.4 & 83.2 & & & \\
\hline & & 14.5 & 10.9 & 70.2 & 134 & & \\
\hline & & 44 & 11.2 & 61.7 & & 135 & 217 \\
\hline \multirow[t]{4}{*}{1000} & 20 & 1 & 10.3 & 91.6 & & & \\
\hline & & 5 & 10.6 & 83.1 & & & \\
\hline & & 14.5 & 11.0 & 71.2 & 82 & & \\
\hline & & 44 & 11.2 & 62.7 & & 135 & 175 \\
\hline
\end{tabular}


CHAPTER 6: NANO-STRUCTURED CALCIUM SILICATE FROM GEOTHERMAL WATER

\begin{tabular}{|c|c|c|c|c|c|c|c|}
\hline \multirow{2}{*}{$\begin{array}{l}\text { Calcium } \\
\text { added } \\
(\mathrm{mg} / \mathrm{L})\end{array}$} & \multirow{2}{*}{$\begin{array}{c}\text { Vol. } \\
\mathrm{NaOH} \\
(\mathrm{mL})\end{array}$} & \multirow{2}{*}{$\begin{array}{l}\text { Time } \\
\text { (min) }\end{array}$} & \multirow[t]{2}{*}{$\mathrm{pH}$} & \multirow{2}{*}{$\begin{array}{l}\text { Temp } \\
\left({ }^{\circ} \mathrm{C}\right)\end{array}$} & \multirow{2}{*}{$\begin{array}{l}{\left[\mathrm{SiO}_{2}\right]_{\mathrm{m}}} \\
(\mathrm{mg} / \mathrm{L})\end{array}$} & \multicolumn{2}{|c|}{ Oil absorption (g/100 g) } \\
\hline & & & & & & $\begin{array}{c}\text { Water } \\
\text { washed }\end{array}$ & 2EE washed \\
\hline \multirow[t]{4}{*}{1000} & 25 & 1 & 10.4 & 91.5 & & & \\
\hline & & 5 & 10.6 & 83.6 & & & \\
\hline & & 15 & 11.0 & 71.0 & 109 & & \\
\hline & & 53 & 11.2 & 61.4 & & 135 & 195 \\
\hline \multirow[t]{4}{*}{1200} & 10 & 1 & 10.1 & 89.0 & & & \\
\hline & & 7.5 & 10.6 & 76.5 & & & \\
\hline & & 14.5 & 10.9 & 67.4 & & & \\
\hline & & 50 & 11.3 & 57.0 & 138 & 139 & 186 \\
\hline \multirow[t]{4}{*}{1200} & 15 & 1.5 & 10.3 & 90.6 & & & \\
\hline & & 5 & 10.6 & 82.0 & & & \\
\hline & & 14 & 11.1 & 67.7 & 51 & & \\
\hline & & 47 & 11.4 & 57.8 & & 138 & 137 \\
\hline \multirow[t]{4}{*}{1200} & 20 & 1 & 10.5 & 89.5 & & & \\
\hline & & 5 & 10.9 & 78.9 & & & \\
\hline & & 14.5 & 11.3 & 63.7 & 56 & & \\
\hline & & 44 & 11.5 & 55.8 & & 134 & 157 \\
\hline \multirow[t]{4}{*}{1200} & 25 & 1 & 10.5 & 91.7 & & & \\
\hline & & 5 & 10.8 & 82.5 & & & \\
\hline & & 14.5 & 11.2 & 69.5 & 85 & & \\
\hline & & 41 & 11.3 & 61.9 & & 142 & 167 \\
\hline
\end{tabular}

The powder X-ray diffractograms of all samples showed the presence of calcium carbonate (PDF: 05-0586) as well as some unreacted calcium hydroxide (PDF: 44-1481) for materials precipitated with the largest amounts of calcium. An example of this is Figure 6.19 which is the equivalent of K13 (from the first series of work) in terms of the amounts of both calcium hydroxide and sodium hydroxide added (the XRD of which is given in Figure $6.8)$. 


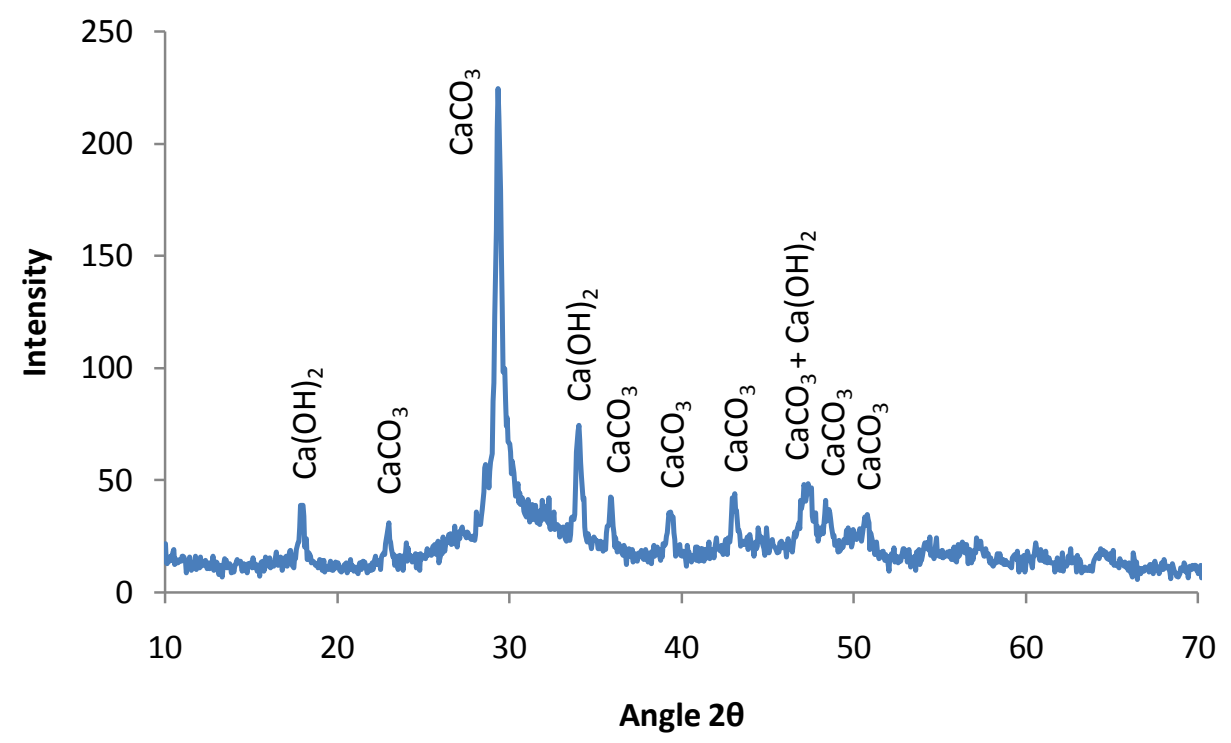

Figure 6.19: X-ray diffractogram of material prepared with the equivalent conditions of K13 (1000 mg/L calcium and $20 \mathrm{~mL} 25 \% \mathrm{NaOH}$ ).

The effect of treating the materials with 2-ethoxyethanol had mixed results upon the oil absorption capacities (Figure 6.20). Some samples improved markedly with the treatment while for others there was no measurable difference. The varying proportion of calcium carbonate in the samples is an important factor in determining the potential improvement in oil absorption capacity the treatment can achieve as calcium carbonate has no pores for 2ethoxyethanol to prevent collapsing. For example, a sample that had a large proportion of its mass consisting of calcium carbonate would have a smaller improvement in its oil absorption capacity by treating with it 2-ethoxyethanol, than a sample that contained less calcium carbonate as this would consist of more nano-structured calcium silicate that is effected. As shown by the materials previously produced, there is no obvious relationship between the proportion of calcium carbonate that forms and the reaction conditions, therefore the effect of 2-ethoxyethanol also has no obvious relationship with reaction conditions. 


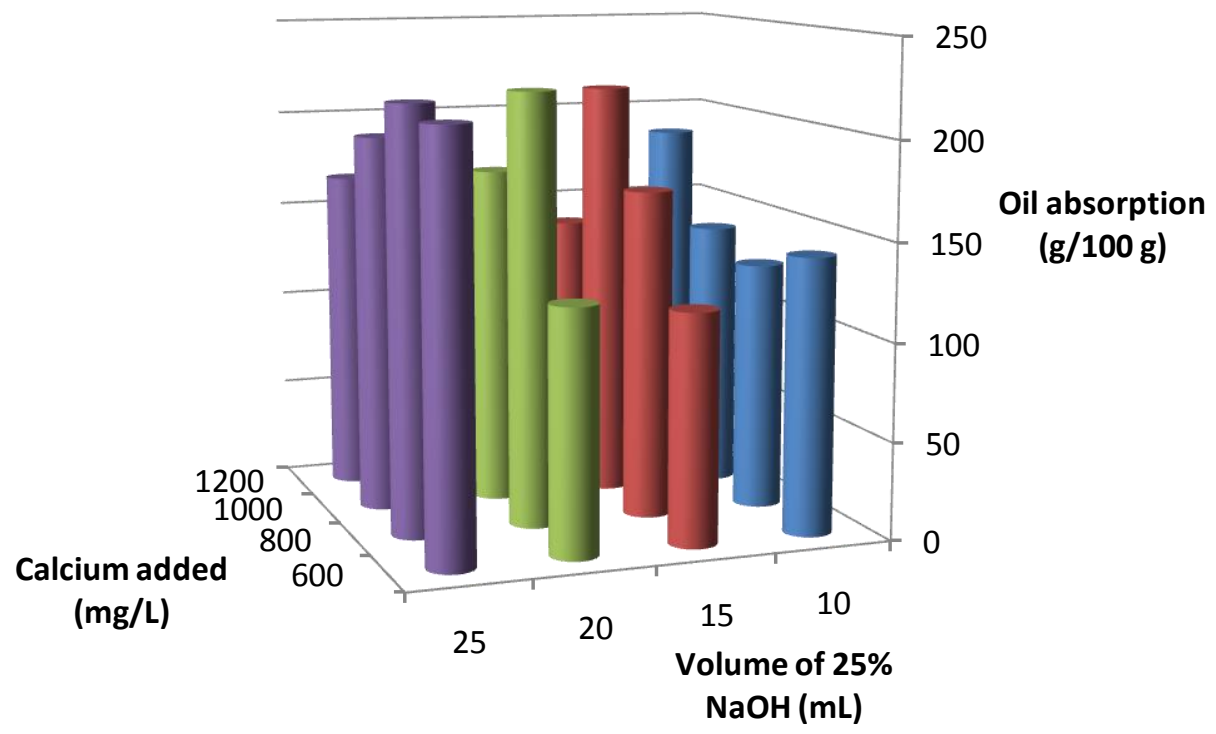

Figure 6.20: Oil absorption capacities of 2-ethoxyethanol washed materials precipitated from geothermal water at Kawerau from second series of experiments.

In conclusion, the use of geothermal water as the monomeric silica source from the Kawerau and Rotokawa fields resulted in the co-precipitation of nanostructured calcium silicate and calcium carbonate. Due to the large proportion of calcium carbonate formed (ca. 50\% in many cases), the mixed material possessed poorer physical properties in terms of oil absorption capacity and surface area, than materials produced using sodium silicate as a monomeric silica source. However, some of the materials produced did have oil absorption capacities in excess of $200 \mathrm{~g} / 100 \mathrm{~g}$ with a maximum of over $250 \mathrm{~g} / 100 \mathrm{~g}$ achieved. These could find use in applications where the higher oil absorption capacity would not be necessary. Ground calcium carbonate (GCC) and precipitated calcium carbonate (PCC) are currently used as paper fillers because of their optical properties, therefore if the properties of the coprecipitated calcium carbonate meet the requirements of an application, its presence may not necessarily be negative.

The remaining monomeric silica concentration following precipitation was below the solubility of silica at room temperature for the $\mathrm{pH}$ of the water in many cases. This was particularly true when larger amounts of calcium was added and would therefore reduce the formation of any silica scale if the thermal energy of the water was to be used. The precipitation of calcium 
carbonate from the water would also decrease the amount of scale that this forms. The arsenic content of the materials produced was found to be below the allowable levels for the applications considered, although this would also need to be measured for different operating conditions.

The inability to reproduce the earlier results at the Kawerau field also raised some issues that need to be addressed. The chemical composition of geothermal water can vary according to the rate of water extraction from the field and the rate at which that the water is replaced (by either natural methods such as ground water seeping down into the resource, or by artificial methods such as reinjecting water back into the resource). If the rate of water extraction from the field is high, the addition of cooler water into the resource can decrease the temperature of the whole resource, affecting the solubility of different species in the system. A minimum requirement for the production of material with consistent chemical and physical properties from the geothermal water over time would be the ability to constantly monitor the composition of the water so that reaction conditions would be known in advance. Ideally, a relationship should exist between the operators of the field and those involved in producing material from the water so that any changes in operation may be known in advance and the effects of the changes determined. Indeed, given that the ideal outcome of using geothermal water is not just to precipitate a material that has an applicable use, but to also allow greater use of the thermal energy of the water, collaboration between both parties would be necessary.

One potential method for using geothermal water to produce a higher quality material while still removing the silica from solution could be to use the water for the reinforcement process only, with the base material prepared using sodium silicate as the monomeric silica source. This could have several advantages in that as a large concentration of calcium ions is not introduced to the fluid, the formation of substantial amounts of calcium carbonate or calcium arsenate would not occur. Also, large volumes of fluid would not need to be stored as the reinforcement process is very rapid and less acid would be required for the process as the $\mathrm{pH}$ of geothermal water is relatively low. 


\section{Chapter 7}

\section{CONCLUSIONS}

Nano-structured calcium silicate is a highly porous material with a large surface area that is formed as a result of the reaction of calcium cations with monomeric silica at room temperature. It is likely that these physical properties will enable this material to perform better than other silicas and silicates in certain applications, and so understanding the material to a greater extent and maximising these properties were of interest. The porosity of the material arises from an open framework of platelets that develops following the precipitation reaction. The formation of this structure is the result of a selforganisation that requires no templating agents. During the development of the framework, an ordering of the structure at an atomic scale also occurs, as observed by ${ }^{29} \mathrm{Si} \mathrm{NMR}$. While the structure does increase in order, the final product remains highly disordered and has an X-ray diffraction pattern with few broad peaks similar to those observed for other hydrated calcium silicate. It is therefore thought that like these calcium silicate hydrates, nano-structured calcium silicate has a structure related to the crystalline mineral tobermorite, that also has a degree of disorder in the structure. The structure of tobermorite consists of layers made up of planes of seven-co-ordinate calcium between long silicate chains of three tetrahedra repeating units (dreierketten) with the disorder of the structure due to the alignment of these layers. In the crystalline mineral, these layers are separated by $1.4,1.1$ or $0.93 \mathrm{~nm}$ depending upon the degree of hydration and can contain water molecules and calcium ions. Typical features of these hydrated calcium silicates are a high degree of inherent disorder and a variation in their composition.

A batch process was used for the studies where reaction conditions were varied and only a relatively small amount of product was required for analysis. Based 
upon knowledge gained from reactions using the batch process, a continuous process was developed that used a small reaction vessel to allow greater control of reaction conditions so that larger quantities of consistent material could be produced. A monomeric silica concentration of $0.43 \mathrm{~mol} / \mathrm{L}$ was typically used for the production of nano-structured calcium silicate at volumes ranging from 0.05 to $150 \mathrm{~L}$. While a wide range of calcium to silicon ratios can be used to form nano-structured calcium silicate, it has been found that a ratio of 0.9 to 1.1 will produce a material with the largest oil absorption capacity and specific surface area. It has also been found that carrying out the reaction at ca. $\mathrm{pH} 12.5$ produces a material with the largest pore volume, with the $\mathrm{pH}$ being controlled through the addition of hydrochloric acid in the more common method where calcium hydroxide is used as the calcium source or through the addition of sodium hydroxide when calcium chloride is used. The waste water from the production of nano-structured calcium silicate contains very low concentrations of both monomeric silica and calcium cations and is alkaline. Most of the sodium and chloride ions can be washed from the pores and are therefore the species that are present in the highest concentrations.

Like silica gels and precipitated silica, the open framework structure of nanostructured calcium silicate has been found to collapse upon the removal of water from the pores during the drying process due to forces as a result of surface tension. If the structure collapses completely, the oil absorption capacity can decrease from ca. $600 \mathrm{~g} / 100 \mathrm{~g}$ to $120 \mathrm{~g} / 100 \mathrm{~g}$ which is therefore a substantial problem in producing a material for applications whereby this property is of importance. As such, three methods have been developed to maintain this property upon drying. The replacement of the water in the pores by 2-ethoxyethanol prevents the structure of the material from collapsing when it is dried, as this solvent has a lower surface tension than water. The water in the pores is replaced by washing a plug flow of the solvent through the filtercake of the silicate. The result of this is a material with an oil absorption capacity of over $600 \mathrm{~g} / 100 \mathrm{~g}$ and a surface area in excess of $500 \mathrm{~m}^{2} / \mathrm{g}$. The 2 ethoxyethanol that remains bound to the material following drying at $110{ }^{\circ} \mathrm{C}$ can subsequently be exchanged for water that still causes the collapse of the 
structure when removed and therefore places limits upon the possible applications of this material.

Another method developed for preventing the structure from collapsing upon drying involves polymerising silica upon the structure, mainly in the areas where the platelets intersect to reinforce the weakest points of the framework. The addition of $0.6 \mathrm{~mol}$ monomeric silica per $1 \mathrm{~mol}$ monomeric silica used to form the nano-structured calcium silicate and subsequent adjustment of the $\mathrm{pH}$ to ca. $\mathrm{pH} 10$, causes essentially all of the monomeric silica added to be polymerised onto the material. The resulting reinforced nano-structured calcium silicate has an oil absorption capacity of ca. $400 \mathrm{~g} / 100 \mathrm{~g}$. It is possible to repeatedly remove water form the pores of this material without causing any collapse of the more robust structure. A pilot plant based upon the continuous process was successfully operated to produce this reinforced nano-structured calcium silicate on a $250 \mathrm{~L}$ scale.

The final method developed that can prevent the collapse of the structure upon drying, was through lowering the $\mathrm{pH}$ of the slurry with the oil absorption capacity of these materials reaching ca. $350 \mathrm{~g} / 100 \mathrm{~g}$. This material was developed for applications where the alkalinity could be problematic, such as paper fillers for grades of paper containing high proportions of mechanical pulp. The lowering of the $\mathrm{pH}$ of nano-structured calcium silicate slurries to neutral conditions also had a significant effect upon the composition of the materials as the calcium in the structure was exchanged for protons. The ratio of calcium to silicon in the material decreased from ca. 1.0 to 0.1 when the $\mathrm{pH}$ was lowered to 7. In order to recover some of the calcium removed from the structure, a recycling process was devised whereby the filtrate of the reaction mixture following the neutralisation of the slurry was used to provide a proportion of the calcium for the preparation of further nano-structured calcium silicate. This cycle of neutralising the slurry, recovering the filtrate, preparing further material, and neutralising this slurry was repeated four times as a proof of concept that this process could produce material with constant properties. 
The use of geothermal water as a source of monomeric silica for the preparation of nano-structured calcium silicate is seen as a potential technique for preventing the formation of scale when this fluid is used. Geothermal water can reach temperatures in excess of $300{ }^{\circ} \mathrm{C}$ and the thermal energy of this resource is used for electricity generation. The reduction of the fluid's temperature during the utilisation of the thermal energy, results in the water becoming supersaturated in silica as the solubility consequently decreases. The result of this is the formation of a hard rock-like scale onto the pipes through which the water flows. The only currently used solution to this problem is to limit the amount of thermal energy removed from the water and therefore reduce the amount of scale that forms. By using the precipitation of nanostructured calcium silicate as a method to remove the majority of silica from the fluid, a greater proportion of the thermal energy of the water can be used for electricity generation and a useful material is produced. Nano-structured calcium silicate was successfully prepared using geothermal water at Kawerau with the material produced possessing pore volumes that exceeded many commercially available precipitated silicas, with a maximum oil absorption capacity of $250 \mathrm{~g} / 100 \mathrm{~g}$ achieved. The properties of the materials prepared were however limited by the co-precipitation of calcium carbonate that exceeded $50 \%$ by weight of the material produced in some cases. The formation of calcium carbonate was due to the high dissolved carbon dioxide content of the water and the alkaline reaction conditions used. Another problem encountered while preparing nano-structured calcium silicate with geothermal water was the lack of reproducibility of results during subsequent work at the geothermal field. This was most likely due to a change in the operating conditions of the resource that altered the composition of the fluid. This would need to be addressed if a material with consistent composition and properties were to be produced.

The properties of the nano-structured calcium silicates produced in this work, particularly the large pore volume and specific surface area, seem to provide the material with the potential to replace some currently used materials in certain applications. Following the ability to produce this material with 
CHAPTER 7: CONCLUSIONS

optimised properties on a larger scale, further investigations are being performed by other members of the research group on applications testing to measure the performance of the material and as well as modifying the material to carry out specific functions. 


\section{References}

Alexander, G. B., Broge, E. C., \& Iler, R. K. (1956). Reinforced and esterified silica gels. US: (E. I. du Pont de Nemours \& Co.). US patent 2765242.

Aylward, G. H., \& Findlay, T. J. V. (2002) SI Chemical Data. (5 ${ }^{\text {th }}$ Edition), Wiley, 30

Athanasopoulos, N. Flame Methods Manual for Atomic Absorption. Victoria, Australia: GBC Scientific Equipment Pty Ltd.

Beck, J. S., Vartuli, J. C., Roth, W. J., Leonowicz, M. E., Kresge, C. T., Schmitt, K. D., et al. (1992). A new family of mesoporous molecular sieves prepared with liquid crystal templates. Journal of the American Chemical Society, 114(27), 10834-10843.

Bickerman, J. J. (1948). Surface Chemistry for Industrial Research. Academic Press, New York

Bonaccorsi, E., Merlino, S., \& Kampf, A. R. (2005). The crystal structure of tobermorite 14 .ANG. (plombierite), a C-S-H phase. Journal of the American Ceramic Society, 88(3), 505-512.

Borrmann, T. (2000). Personal communication.

Borrmann, T., \& Johnston, J. H. (2002). Catalysts and the hydroformylation of olefins, NZ patent 2002048039

Burdett, J. K. (1988). Perspectives in structural chemistry. Chemical Reviews (Washington, DC, United States), 88(1), 3-30.

Cairns, M. J., Borrmann, T., Hoell, W. H., \& Johnston, J. H. (2006). A study of the uptake of copper ions by nanostructured calcium silicate. Microporous and Mesoporous Materials, 95(1-3), 126-134.

Chen, J. J., Thomas, J. J., Taylor, H. F. W., \& Jennings, H. M. (2004). Solubility and structure of calcium silicate hydrate. Cement and Concrete Research, 34(9), 1499-1519.

Cong, X., \& Kirkpatrick, R. J. (1996). ${ }^{29}$ Si MAS NMR Study of the Structure of Calcium Silicate Hydrate. Advanced Cement Based Materials, 3(3/4), 144156.

Cong, X., \& Kirkpatrick, R. J. (1996a). ${ }^{29} \mathrm{Si}$ and ${ }^{17} \mathrm{O}$ NMR Investigation of the Structure of Some Crystalline Calcium Silicate Hydrates. Advanced Cement Based Materials, 3(3/4), 133-143. 
Coradin, T., \& Lopez, P. J. (2003). Biogenic silica patterning: simple chemistry or subtle biology? ChemBioChem, 4(4), 251-259.

Durham, G. (1997). Toxic Elements and their Compounds in Graphic Materials: Guidelines for Public Health Services. Wellington, NZ: Ministry of Health.

Earnest, C. M. (1988). The modern thermogravimetric approach to compositional analysis of materials. ASTM Special Technical Publication 997: Compositional Analysis by Thermogravimetry

Ellis, A. J., \& Mahon, W. A. J. (1977). Chemistry and Geothermal Systems. Academic Press.

Evans, B., \& Slozer, M. (2003). Neutral groundwood papers: practical and chemical aspects. Preprints - International Paper and Coating Chemistry Symposium, 5th, Montreal, QC, Canada, June 16-19, 2003, 43-46.

Falcone, J. S. (2000). Silicon compounds: Synthetic inorganic silicates. KirkOthmer Encyclopedia of Chemical Technology (4th Edition), 22, 1-30.

Flanigen, E. M. (2001). Zeolites and molecular sieves: An historical perspective. Studies in Surface Science and Catalysis, 137(Introduction to Zeolite Science and Practice (2nd Edition)), 11-35.

Flanigen, E. M., Bennett, J. M., Grose, R. W., Cohen, J. P., Patton, R. L., Kirchner, R. M., et al. (1978). Silicalite, a new hydrophobic crystalline silica molecular sieve. Nature (London, United Kingdom), 271(5645), 512-516.

Fujii, K., \& Kondo, W. (1981). Heterogeneous equilibrium of calcium silicate hydrate in water at 30 DegC. Journal of the Chemical Society, Dalton Transactions: Inorganic Chemistry (1972-1999)(2), 645-651.

Fyfe, C. A. (1983). Solid State NMR for Chemists. C.F.C. Press, Guelph, Ont.

Gibbs, G. V., Downs, J. W., \& Boisen, M. B., Jr. (1994). The elusive SiO bond. Reviews in Mineralogy, 29(SILICA), 331-368.

Gill, R. A. (2005). Fillers for Papermaking, from http://www.specialtyminerals.com/publications/paper-filling-smipublications/

Hall, M. (2004). Existing and Potential Geothermal Resource for Electricity Generation.

Hamid, S. A. (1981). The crystal structure of the 11 .ANG. natural tobermorite $\mathrm{Ca}_{2.25}\left[\mathrm{Si}_{3} \mathrm{O}_{7.5}(\mathrm{OH})_{1.5}\right] .1 \mathrm{H}_{2} \mathrm{O}$. Zeitschrift fuer Kristallographie, 154(3-4), 189-198. 
Harper, R. T. (1994). Extraction of amorphous silica from geothermal water and its application to improve newsprint quality., Victoria University of Wellington, Wellington.

Harper, R. T., Johnston, J. H., \& Wiseman, N. (1997). Controlled precipitation of amorphous silica from geothermal fluids or other aqueous media containing silicic acid (pp. 57 pp , Cont -in-part of U S 55,200,165). US patent 5595717

Himmel, B., Gerber, T., \& Neumann, H. G. (1985). X-ray diffraction investigations of differently prepared amorphous silicas. Physica Status Solidi A Applied Research, 88(2), K127-K130.

Holland, B. T., Abrams, L., \& Stein, A. (1999). Dual Templating of Macroporous Silicates with Zeolitic Microporous Frameworks. Journal of the American Chemical Society, 121(17), 4308-4309.

Hubbe, M. A. (2006). Paper. Kirk-Othmer Encyclopedia of Chemical Technology (5th Edition), 18, 89-132.

HunterLab (1996). CIE L*a*b* color scale, Applications Note, 8(7), 1-4. (Can be downloaded from: http://www.hunterlab.com)

HunterLab, (2001). The basics of color perception and measurement, Version 1.4, http://www.hunterlab.com/colorbasics_shows.php

Iler, R. K. (1979). The Chemistry of Silica: Solubility, Polymerization, Colloid and Surface Properties and Biochemistry: John Wiley and Son, Chichester, Engl.

Imhof, A., \& Pine, D. J. (1997). Ordered macroporous materials by emulsion templating. Nature (London), 389(6654), 948-951.

Johnston, J. H. (2007). Personal communication.

Johnston, J.H., Borrmann,T. \& McFarlane, A.J. (2006). Nano-structured Silicate, Functionalised Forms Thereof, Preparation and Uses. NZ Patent Specification No. 537747.; International PCT Application PCT/NZ2006/000003.

Johnston, J. H., McFarlane, A. J., \& Borrmann, T. (2002). New high performance calcium-silica materials for filled and specialty papers. Appita Annual Conference Proceedings, 56th, 453-457.

Johnston, J. H., McFarlane, A. J., Borrmann, T., \& Moraes, J. (2004). Nanostructured silicas and silicates - new materials and their applications in paper. Current Applied Physics, 4(2-4), 411-414. 
Kokotailo, G. T., Lawton, S. L., Olson, D. H., \& Meier, W. M. (1978). Structure of synthetic zeolite ZSM-5. Nature (London, United Kingdom), 272(5652), 437-438.

Kresge, C. T., Leonowicz, M. E., Roth, W. J., Vartuli, J. C., \& Beck, J. S. (1992). Ordered mesoporous molecular sieves synthesized by a liquid-crystal template mechanism. Nature (London, United Kingdom), 359(6397), 710-712.

Kuhl, G. H. \& Kresge, C. T. (2000). Molecular Sieves. Kirk-Othmer Encyclopedia of Chemical Technology (4th Edition), 16, 888-925.

Li, F., Wang, Z., Ergang, N. S., Fyfe, C. A., \& Stein, A. (2007). Controlling the Shape and Alignment of Mesopores by Confinement in Colloidal Crystals: Designer Pathways to Silica Monoliths with Hierarchical Porosity. Langmuir, 23(7), 3996-4004.

Lide, D. R. (2005). CRC Handbook of Chemistry and Physics, 86th Edition.

MacKenzie, K. J. D., \& Smith, M. E. (2002). Multinuclear Solid-State Nuclear Magnetic Resonance of Inorganic Materials, Pergamon Materials Series, v. 6.

Manzano, H., Ayuela, A., \& Dolado, J. S. (2007). On the formation of cementitious C-S-H nanoparticles. Journal of Computer-Aided Materials Design, 14(1), 45-51.

Maple, M. (2006). Pretty Useful. Chemistry \& Industry, (8), 19-21.

McGraw-Hill (2003) McGraw-Hill Dictionary of Materials Science. McGraw-Hill, New York

McNaught, A. D., \& Wilkinson, A. (1997). Compendium of Chemical Terminology, 2nd Edition.

Merlino, S., Bonaccorsi, E., \& Armbruster, T. (1999). Tobermorites: their real structure and order-disorder (OD) character. American Mineralogist, 84(10), 1613-1621.

Merlino, S., Bonaccorsi, E., \& Armbruster, T. (2000). The real structures of clinotobermorite and tobermorite 9 .ANG.: OD character, polytypes, and structural relationships. European Journal of Mineralogy, 12(2), 411429.

Merlino, S., Bonaccorsi, E., \& Armbruster, T. (2001). The real structure of tobermorite 11.ANG.: normal and anomalous forms, OD character and polytypic modifications. European Journal of Mineralogy, 13(3), 577-590. 
Meyer, S. J. (1996). The development and optimisation of the process chemistry to extract an amorphous silica product from geothermal water to enhance paper quality Victoria University of Wellington, Wellington.

Mink, R. (2005). Geothermal energy. Kirk-Othmer Encyclopedia of Chemical Technology (5th Edition), 12, 521-547.

Neumann, D., Raverty, W. D., \& Vanderhoek, N. (2005). Nanostructured materials and their use in ink receptor layers. Appita Annual Conference and Exhibition, 59th, 225-229.

Packter, A. (1986). Precipitation of alkaline-earth metal silicate hydrates from aqueous solution: ionic equilibria, crystalline phases and precipitation mechanisms. Crystal Research and Technology, 21(5), 575-585.

Pauling, L. (1929). The principles determining the structure of complex ionic crystals. Journal of the American Chemical Society, 51, 1010-1026.

Pauling, L. (1980). The nature of silicon-oxygen bonds. American Mineralogist, 65(3-4), 321-323.

Roller, P. S., \& Ervin, G., Jr. (1940). The system calcium oxide-silica-water at 30 Deg. The association of silicate ion in dilute alkaline solution. Journal of the American Chemical Society, 62, 461-471.

Schroden, R. C., \& Stein, A. (2004). 3D ordered macroporous materials. Colloids and Colloid Assemblies, 465-493.

Shaw, S., Clark, S. M., \& Henderson, C. M. B. (2000). Hydrothermal formation of the calcium silicate hydrates, tobermorite $\left(\mathrm{Ca}_{5} \mathrm{Si}_{6} \mathrm{O}_{16}(\mathrm{OH}) 2.4 \mathrm{H}_{2} \mathrm{O}\right)$ and xonotlite $\left(\mathrm{Ca}_{6} \mathrm{Si}_{6} \mathrm{O}_{17}(\mathrm{OH})_{2}\right)$ : an in situ synchrotron study. Chemical Geology, 167(1-2), 129-140.

Shaw, S., Henderson, C. M. B., \& Komanschek, B. U. (2000a). Dehydration /recrystallization mechanisms, energetics, and kinetics of hydrated calcium silicate minerals: an in situ TGA/DSC and synchrotron radiation SAXS/WAXS study. Chemical Geology, 167(1-2), 141-159.

Smook, G., \& Editor. (1992). Handbook for Pulp \& Paper Technologists (2nd ed.).

Song, W., Justice, R. E., Jones, C. A., Grassian, V. H., \& Larsen, S. C. (2004). SizeDependent Properties of Nanocrystalline Silicalite Synthesized with Systematically Varied Crystal Sizes. Langmuir, 20(11), 4696-4702.

Song, W., Justice, R. E., Jones, C. A., Grassian, V. H., \& Larsen, S. C. (2004). Synthesis, Characterization, and Adsorption Properties of Nanocrystalline ZSM-5. Langmuir, 20(19), 8301-8306. 
Southam, D. C., Lewis, T. W., McFarlane, A. J., \& Johnston, J. H. (2004). Amorphous calcium silicate as a chemisorbent for phosphate. Current Applied Physics, 4(2-4), 355-358.

Steinour, H. H. (1947). The system CaO-SiO2-H2O and the hydration of the calcium silicates. Chem. Rev., 40, 391-460.

Taylor, H. F. W. (1950). Hydrated calcium silicates. I. Compound formation at ordinary temperatures. Journal of the Chemical Society, 3682-3690.

Taylor, H. F. W. (1953). Hydrated calcium silicates. V. Water content of calcium silicate hydrate I. Journal of the Chemical Society, 163-171.

Taylor, H. F. W. (1964). The Chemistry of Cements: Vol 1, Academic Press, New York, 183.

Taylor, H. F. W. (1993). Nanostructure of C-S-H: current status. Advanced Cement Based Materials, 1(1), 38-46.

Valdes, M. G., Perez-Cordoves, A. I., \& Diaz-Garcia, M. E., (2006). Zeolites and zeolite-based materials in analytical chemistry. Trends in Analytical Chemistry, 25(1), 24-30

Valtchev, V. (2002). Preparation of regular macroporous structures built of intergrown silicalite-1 nanocrystals. Journal of Materials Chemistry, 12(6), 1914-1918.

Velev, O. D., Jede, T. A., Lobo, R. F., \& Lenhoff, A. M. (1997). Porous silica via colloidal crystallization. Nature (London), 389(6650), 447-448.

Waddell, W. H. (2006). Silica, amorphous. Kirk-Othmer Encyclopedia of Chemical Technology (5th Edition), 22, 380-406.

Walsh, D., \& Mann, S. (1995). Fabrication of hollow porous shells of calcium carbonate from self-organizing media. Nature (London), 377(6547), 320-323.

Weldes, H. H., \& Lange, K. R. (1969). Properties of soluble silicates. Industrial and Engineering Chemistry, 61(4), 29-44.

Wu, M., Fujiu, T., \& Messing, G. L. (1990). Synthesis of cellular inorganic materials by foaming sol-gels. Journal of Non-Crystalline Solids, 121(1-3), 407-412.

Wypych, G. (1999). Handbook of Fillers (2nd Edition). ChemTec Publishing

Yu, P., Kirkpatrick, R. J., Poe, B., McMillan, P. F., \& Cong, X. (1999). Structure of calcium silicate hydrate (C-S-H): near-, mid-, and far-infrared spectroscopy. Journal of the American Ceramic Society, 82(3), 742-748. 


\section{Appendix A - Data for graphs}

Data used for graphs that is not already included in the main text.

\section{Data for Figure 3.1}

Concentration of reactants in remaining in solution following precipitation.

\begin{tabular}{lcc}
\multicolumn{3}{l}{ Reaction conditions } \\
\cline { 1 - 1 } \multicolumn{3}{l}{ Total volume: $1 \mathrm{~L}$} \\
{$\left[\mathrm{SiO}_{2}\right]_{\mathrm{m}}$} & 0.319 & $\mathrm{~mol} / \mathrm{L}$ \\
{$[\mathrm{Ca}]$} & 0.313 & $\mathrm{~mol} / \mathrm{L}$ \\
{$[\mathrm{HCl}]$} & 0.170 & $\mathrm{~mol} / \mathrm{L}$ \\
& & \\
\hline Time & {$\left[\mathrm{Ca}^{2+}\right]$} & {$\left[\mathrm{SiO}_{2}\right]_{\mathrm{m}}$} \\
$(\mathrm{min})$ & $(\mathrm{mg} / \mathrm{L})$ & $(\mathrm{mg} / \mathrm{L})$ \\
\hline 5 & 330.0 & 5.5 \\
15 & 422.5 & 1.3 \\
30 & 427.5 & 5.5 \\
45 & 395.0 & 5.5 \\
60 & 340.0 & 7.9 \\
90 & 220.0 & 13.4 \\
120 & 177.5 & 29.2 \\
150 & 177.5 & 42.6 \\
180 & 197.5 & 44.9 \\
1480 & 179.5 & 15.0 \\
\hline
\end{tabular}

\section{Data for Figures 3.2 \& 3.10}

Composition of product during ageing.

\section{Reaction conditions}

Total volume: $5 \mathrm{~L}$

$\begin{array}{lll}{\left[\mathrm{SiO}_{2}\right]_{\mathrm{m}}} & 0.579 & \mathrm{~mol} / \mathrm{L} \\ {[\mathrm{Ca}]} & 0.623 & \mathrm{~mol} / \mathrm{L} \\ {[\mathrm{HCl}]} & 0.316 & \mathrm{~mol} / \mathrm{L}\end{array}$

\begin{tabular}{cccccc}
\hline $\begin{array}{c}\text { Time } \\
(\mathrm{min})\end{array}$ & Wash & $\mathrm{Ca} / \mathrm{Si}$ & LOI/Si & $\begin{array}{c}\mathrm{OA} \\
(\mathrm{g} / 100 \mathrm{~g})\end{array}$ & $\begin{array}{c}\mathrm{SA} \\
\left(\mathrm{m}^{2} / \mathrm{g}\right)\end{array}$ \\
\hline 30 & $2 \mathrm{ME}$ & 0.99 & 1.32 & 160 & 70 \\
120 & $2 \mathrm{ME}$ & 0.99 & 1.47 & 265 & 169 \\
300 & $2 \mathrm{ME}$ & 0.99 & 1.70 & 400 & 255 \\
300 & water & 0.98 & 1.36 & 125 & 43 \\
\hline
\end{tabular}




\section{Data for Figure 3.9}

Changes in physical properties over time during ageing. Samples 2-ethoxyethanol washed.

Reaction conditions

Total volume: $1 \mathrm{~L}$

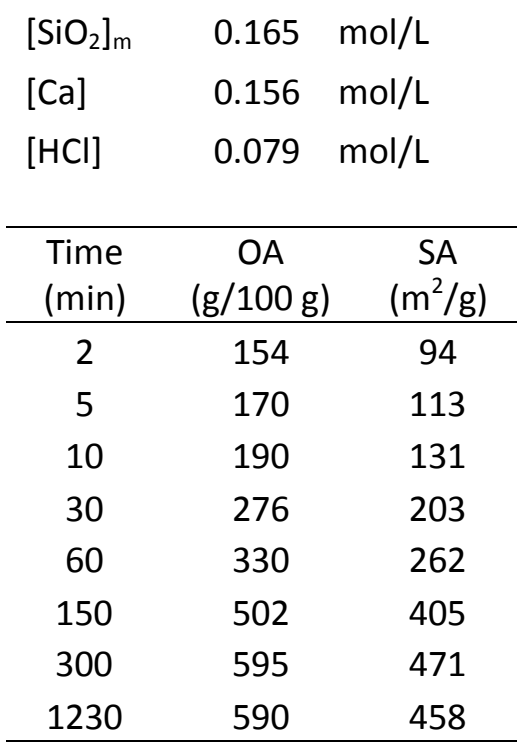

Data for Figure 3.21

Effect of calcining upon physical properties.

Samples washed with 2-ethoxyethanol.

Reaction conditions

Total volume: $1 \mathrm{~L}$

$\left[\mathrm{SiO}_{2}\right]_{\mathrm{m}} \quad 0.165 \mathrm{~mol} / \mathrm{L}$

[Ca] $\quad 0.156 \mathrm{~mol} / \mathrm{L}$

$[\mathrm{HCl}] \quad 0.079 \mathrm{~mol} / \mathrm{L}$

\begin{tabular}{ccc}
\hline $\begin{array}{c}\text { Temp. } \\
\left({ }^{\circ} \mathrm{C}\right)\end{array}$ & $\begin{array}{c}\text { OA } \\
(\mathrm{g} / 100 \mathrm{~g})\end{array}$ & $\begin{array}{c}\mathrm{SA} \\
\left(\mathrm{m}^{2} / \mathrm{g}\right)\end{array}$ \\
\hline 110 & 534 & 472 \\
300 & 483 & 441 \\
600 & 539 & 440 \\
650 & 537 & 404 \\
700 & 470 & 353 \\
750 & 150 & 82 \\
800 & 71 & 9 \\
\hline
\end{tabular}




\section{Data for Figure 4.3}

Relative plug flow volume required for 2-ethoxyethanol treatment

\section{Reaction conditions}

Total

volume: $5 \mathrm{~L}$

$\left[\mathrm{SiO}_{2}\right]_{\mathrm{m}} \quad 0.579 \mathrm{~mol} / \mathrm{L}$

[Ca] $\quad 0.623 \mathrm{~mol} / \mathrm{L}$

$[\mathrm{HCl}] \quad 0.316 \mathrm{~mol} / \mathrm{L}$

\begin{tabular}{cc}
\hline $\begin{array}{c}\mathrm{V}_{\text {plug flow }} \\
/ \mathrm{V}_{\text {slury }}\end{array}$ & $\begin{array}{c}\mathrm{OA} \\
(\mathrm{g} / 100 \mathrm{~g})\end{array}$ \\
\hline 0.83 & 452 \\
0.42 & 418 \\
0.17 & 291 \\
0.08 & 222 \\
\hline
\end{tabular}

Data for Figures 4.4, 4.5, 4.9, \& 4.10

Effect of 2-ethoxyethanol washing upon weight of product and physical and optical properties

\begin{tabular}{cccccccc}
\hline$\% \mathrm{EE}$ & $\begin{array}{c}\mathrm{OA} \\
(\mathrm{g} / 100 \mathrm{~g})\end{array}$ & $\begin{array}{c}\mathrm{SA} \\
\left(\mathrm{m}^{2} / \mathrm{g}\right)\end{array}$ & $\begin{array}{c}\% \mathrm{Wt} \\
\text { gain }\end{array}$ & $\mathrm{L}^{*}$ & $\mathrm{a}^{*}$ & $\mathrm{~b}^{*}$ & $\begin{array}{c}457 \mathrm{~nm} \\
\text { Brightness }\end{array}$ \\
\hline 0 & 126 & 100 & 0.00 & 98.23 & 0.14 & 1.18 & 93.80 \\
1 & 138 & & & & & & \\
10 & 187 & & & & & & \\
20 & 275 & 275 & 2.66 & 97.68 & -0.05 & 0.99 & 92.73 \\
25 & 282 & & & & & & \\
30 & 327 & & 3.55 & & & & \\
40 & 375 & 351 & 4.79 & 96.83 & -0.11 & 0.85 & 90.87 \\
50 & 387 & & & & & & \\
50 & 384 & 351 & 5.85 & 97.17 & -0.09 & 0.93 & 91.57 \\
80 & 428 & 425 & 5.85 & 96.79 & -0.13 & 1.29 & 90.18 \\
100 & 452 & 441 & & 96.44 & -0.06 & 2.02 & 88.40 \\
100 & 434 & & & & & & \\
\hline
\end{tabular}


Data for Figures 4.13, 4.14, \& 4.15

Effects of reinforcement

Reaction conditions

Total volume: $5 \mathrm{~L}$

$\left[\mathrm{SiO}_{2}\right]_{\mathrm{m}} \quad 0.580 \mathrm{~mol} / \mathrm{L}$

[Ca] $\quad 0.620 \mathrm{~mol} / \mathrm{L}$

$[\mathrm{HCl}] \quad 0.340 \mathrm{~mol} / \mathrm{L}$

\begin{tabular}{cccc}
\hline $\begin{array}{c}\text { Reinforcement } \\
(\mathrm{mol} / \mathrm{mol})\end{array}$ & $\begin{array}{c}\mathrm{OA} \\
(\mathrm{g} / 100 \mathrm{~g})\end{array}$ & $\begin{array}{c}\mathrm{Si} / \mathrm{Ca} \\
(\mathrm{mol} / \mathrm{mol})\end{array}$ & $\begin{array}{c}{\left[\mathrm{SiO}_{2}\right]_{\mathrm{m}}} \\
(\mathrm{mg} / \mathrm{L})\end{array}$ \\
\hline 0.0 & 113 & 1.05 & 6.4 \\
0.5 & 181 & 1.46 & 229.8 \\
1.0 & 239.5 & 1.74 & 1562.3 \\
1.5 & 266 & 1.78 & 4016.4 \\
\hline
\end{tabular}

Data for Figures 4.17 \& 4.18

The rate of reinforcement

Reaction conditions

Total volume: $10 \mathrm{~L}$

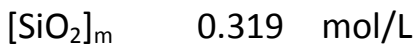

[Ca] $0.312 \mathrm{~mol} / \mathrm{L}$

$[\mathrm{HCl}] \quad 0.169 \mathrm{~mol} / \mathrm{L}$

\begin{tabular}{cccc}
\hline $\begin{array}{c}\text { Time } \\
(\mathrm{min})\end{array}$ & $\begin{array}{c}{\left[\mathrm{SiO}_{2}\right]_{\mathrm{m}}} \\
(\mathrm{mg} / \mathrm{L})\end{array}$ & $\begin{array}{c}\mathrm{OA} \\
(\mathrm{g} / 100 \mathrm{~g})\end{array}$ & $\begin{array}{c}\mathrm{SA} \\
\left(\mathrm{m}^{2} / \mathrm{g}\right)\end{array}$ \\
\hline 5 & 243 & 406 & 300 \\
15 & 242 & 401 & 297 \\
30 & 241 & 377 & 295 \\
45 & 242 & 384 & 276 \\
60 & 237 & 385 & 301 \\
120 & 234 & 421 & 309 \\
5760 & 222 & 399 & 267 \\
\hline
\end{tabular}


Data for Figures 4.19, 4.20, \& 4.21

Effect of reinforcement at ca. $\mathrm{pH} 10$

Reaction conditions

Total volume: $10 \mathrm{~L}$

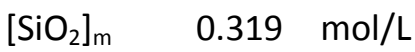

[Ca] $\quad 0.312 \mathrm{~mol} / \mathrm{L}$

$[\mathrm{HCl}] \quad 0.169 \mathrm{~mol} / \mathrm{L}$

\begin{tabular}{ccccc}
\hline $\begin{array}{c}\text { Reinforcement } \\
(\mathrm{mol} / \mathrm{mol})\end{array}$ & $\begin{array}{c}\mathrm{OA} \\
(\mathrm{g} / 100 \mathrm{~g})\end{array}$ & $\begin{array}{c}\mathrm{SA} \\
\left(\mathrm{m}^{2} / \mathrm{g}\right)\end{array}$ & $\begin{array}{c}{\left[\mathrm{SiO}_{2}\right]_{\mathrm{m}}} \\
(\mathrm{mg} / \mathrm{L})\end{array}$ & $\begin{array}{c}\text { Product } \\
\mathrm{Wt}(\mathrm{g})\end{array}$ \\
\hline 0.00 & 183 & 218 & 119 & 1.94 \\
0.14 & 225 & 262 & 144 & 2.10 \\
0.27 & 269 & 308 & 157 & \\
0.41 & 299 & 299 & 173 & 2.46 \\
0.55 & 365 & 287 & 172 & 2.52 \\
0.68 & 376 & 283 & 180 & 2.79 \\
0.82 & 375 & 311 & 238 & 2.86 \\
0.96 & 378 & 297 & 303 & 3.00 \\
1.10 & 404 & 295 & 410 & 3.00 \\
\hline
\end{tabular}

Data for Figures 4.29, 4.30, 4.32, \& 4.33

Effect of acidifying the slurry.

Reaction conditions

Total volume: $5 \mathrm{~L}$

$\left[\mathrm{SiO}_{2}\right]_{\mathrm{m}} \quad 0.579 \mathrm{~mol} / \mathrm{L}$

[Ca] $\quad 0.623 \mathrm{~mol} / \mathrm{L}$

$[\mathrm{HCl}] \quad 0.337 \mathrm{~mol} / \mathrm{L}$

\begin{tabular}{cccccc}
\hline $\mathrm{pH}$ & $\begin{array}{c}\text { Product } \\
\mathrm{Ca} / \mathrm{Si}\end{array}$ & $\begin{array}{c}{\left[\mathrm{Ca}^{2+}\right]} \\
(\mathrm{mg} / \mathrm{L})\end{array}$ & $\begin{array}{c}{\left[\mathrm{SiO}_{2}\right]_{\mathrm{m}}} \\
(\mathrm{mg} / \mathrm{L})\end{array}$ & $\begin{array}{c}\mathrm{OA} \\
(\mathrm{g} / 100 \mathrm{~g})\end{array}$ & $\begin{array}{c}\mathrm{SA} \\
\left(\mathrm{m}^{2} / \mathrm{g}\right)\end{array}$ \\
\hline 12.0 & 0.95 & 600.7 & 14.5 & 113 & 60 \\
10.0 & 0.81 & 4023.3 & 70.7 & 148 & 111 \\
8.5 & 0.55 & 8779.9 & 143.0 & 294 & 260 \\
7.0 & 0.11 & 13996.5 & 123.0 & 367 & 267 \\
6.0 & 0.05 & 14393.2 & 121.3 & 372 & 317 \\
\hline
\end{tabular}


Data for Figures 4.22, 4.23, 4.24, \& 4.25

Altering the amount of reinforcement and the $\mathrm{pH}$ at which it occurs

Reaction conditions

Total volume: $4 \mathrm{~L}$

$\left[\mathrm{SiO}_{2}\right]_{\mathrm{m}} \quad 0.429 \mathrm{~mol} / \mathrm{L}$

[Ca] $\quad 0.469 \mathrm{~mol} / \mathrm{L}$

$[\mathrm{HCl}] \quad 0.253 \mathrm{~mol} / \mathrm{L}$

\begin{tabular}{ccccccc}
\hline $\begin{array}{c}\text { Reinforcement } \\
(\mathrm{mol} / \mathrm{mol})\end{array}$ & $\begin{array}{c}\text { Vol. of } 2 \\
\mathrm{~mol} / \mathrm{L} \\
\mathrm{HCl}(\mathrm{mL})\end{array}$ & $\mathrm{pH}$ & $\begin{array}{c}\mathrm{OA} \\
(\mathrm{g} / 100 \mathrm{~g})\end{array}$ & $\begin{array}{c}\mathrm{SA} \\
\left(\mathrm{m}^{2} / \mathrm{g}\right)\end{array}$ & $\begin{array}{c}{\left[\mathrm{SiO}_{2}\right]_{\mathrm{m}}} \\
(\mathrm{mg} / \mathrm{L})\end{array}$ & $\begin{array}{c}{\left[\mathrm{Ca}^{2+}\right]} \\
(\mathrm{mg} / \mathrm{L})\end{array}$ \\
\hline 0.51 & 3 & 10.1 & 376 & 289 & 249 & 200 \\
& 4 & 9.8 & 397 & 301 & 197 & 500 \\
& 5 & 9.5 & 408 & 285 & 174 & 955 \\
& 6 & 9.4 & 404 & 305 & 160 & 1375 \\
\hline 0.61 & 3 & 10.1 & 378 & 261 & 322 & 130 \\
& 4 & 9.8 & 394 & 291 & 223 & 340 \\
& 5 & 9.6 & 383 & 282 & 186 & 630 \\
& 6 & 9.4 & 406 & 306 & 165 & 1110 \\
\hline 0.71 & 3 & 10.3 & 382 & 284 & 423 & 55 \\
& 4 & 10 & 389 & 271 & 266 & 185 \\
& 5 & 9.7 & 405 & 286 & 200 & 445 \\
& 6 & 9.5 & 403 & 271 & 177 & 840 \\
\hline 0.82 & 3 & 10.5 & 395 & 302 & 544 & 20 \\
& 4 & 10.1 & 410 & 295 & 333 & 90 \\
& 5 & 9.8 & 402 & 290 & 228 & 265 \\
& 6 & 9.6 & 397 & 257 & 184 & 590 \\
\hline
\end{tabular}


Data for Figures 4.39 \& 4.40

Effect of acidifying the material upon the optical propeties.

Reaction conditions

Total volume: $1 \mathrm{~L}$

$\left[\mathrm{SiO}_{2}\right]_{\mathrm{m}} \quad 0.50 \mathrm{~mol} / \mathrm{L}$

[Ca] $\quad 0.75 \mathrm{~mol} / \mathrm{L}$

\begin{tabular}{cccccc}
\hline \multicolumn{7}{c}{ TAPPI } & & & \\
$\mathrm{pH}$ & $\mathrm{L}^{*}$ & $\begin{array}{c}\text { Brightness } \\
457 \mathrm{~nm}\end{array}$ & $\begin{array}{c}\text { Reflectance } \\
580 \mathrm{~nm}\end{array}$ & $\mathrm{a}^{*}$ & $\mathrm{~b}^{*}$ \\
\hline 11.24 & 98.17 & 94.23 & 95.67 & 0.02 & 0.70 \\
10.50 & 98.13 & 94.52 & 95.60 & -0.03 & 0.51 \\
10.00 & 98.35 & 95.16 & 96.00 & 0.02 & 0.31 \\
9.50 & 98.22 & 94.99 & 95.64 & -0.02 & 0.24 \\
9.00 & 98.24 & 95.05 & 95.73 & 0.02 & 0.22 \\
8.50 & 98.14 & 94.80 & 95.40 & 0.00 & 0.21 \\
8.00 & 98.10 & 94.74 & 95.32 & 0.01 & 0.19 \\
7.50 & 97.94 & 94.34 & 94.89 & -0.02 & 0.18 \\
7.00 & 98.37 & 95.90 & 95.85 & -0.03 & -0.19 \\
\hline
\end{tabular}

Data for Figures 4.41 \& 4.42

Recycling filtrate from acidification.

Reaction conditions

\begin{tabular}{|c|c|c|c|c|c|c|}
\hline Total vo & me: & $0.5 \mathrm{~L}$ & & & & \\
\hline$\left[\mathrm{SiO}_{2}\right]_{\mathrm{m}}$ & 0.579 & $\mathrm{~mol} / \mathrm{L}$ & & & & \\
\hline [Ca] & 0.623 & $\mathrm{~mol} / \mathrm{L}$ & (calc & ted) & & \\
\hline $\begin{array}{l}\text { Time } \\
(\min )\end{array}$ & $\begin{array}{c}\text { OA } \\
(\mathrm{g} / 100 \mathrm{~g})\end{array}$ & & $\mathrm{pH}$ & $\begin{array}{c}\text { OA } \\
(\mathrm{g} / 100 \mathrm{~g})\end{array}$ & $\begin{array}{c}\text { SA } \\
\left(\mathrm{m}^{2} / \mathrm{g}\right)\end{array}$ & \\
\hline 5 & 156 & & 11.7 & 391 & 453 & (2EE washed) \\
\hline 30 & 199 & & 11.7 & 129 & 87 & \\
\hline 120 & 347 & & 10.5 & 145 & 81 & \\
\hline 180 & 384 & & 10.0 & 159 & 110 & \\
\hline 240 & 388 & & 9.5 & 171 & 113 & \\
\hline & & & 9.0 & 236 & 207 & \\
\hline & & & 8.5 & 310 & 301 & \\
\hline & & & 8.0 & 331 & 358 & \\
\hline & & & 7.5 & 351 & 347 & \\
\hline & & & 7.0 & 324 & 354 & \\
\hline
\end{tabular}


Data for Figures $5.1 \& \mathbf{5 . 2}$

Effect of varying reactant $\mathrm{Ca} / \mathrm{Si}$ ratio

Reaction conditions (for $0.98 \mathrm{Ca} / \mathrm{Si}$ ratio)

Total volume: $2 \mathrm{~L}$

$\left[\mathrm{SiO}_{2}\right]_{\mathrm{m}} \quad 0.319 \mathrm{~mol} / \mathrm{L}$

[Ca] $\quad 0.312 \mathrm{~mol} / \mathrm{L}$

$[\mathrm{HCl}] \quad 0.169 \mathrm{~mol} / \mathrm{L}$

\begin{tabular}{ccccc}
\hline $\begin{array}{c}\text { Reactant } \\
\mathrm{Ca} / \mathrm{Si}\end{array}$ & $\begin{array}{c}\mathrm{OA} \\
(\mathrm{g} / 100 \mathrm{~g})\end{array}$ & $\begin{array}{c}\mathrm{SA} \\
\left(\mathrm{m}^{2} / \mathrm{g}\right)\end{array}$ & $\begin{array}{c}{\left[\mathrm{SiO}_{2}\right]_{\mathrm{m}}} \\
(\mathrm{mg} / \mathrm{L})\end{array}$ & $\begin{array}{c}{\left[\mathrm{Ca}^{2+}\right]} \\
(\mathrm{mg} / \mathrm{L})\end{array}$ \\
\hline 1.29 & 475 & 442 & 3.8 & 557.5 \\
1.22 & 526 & 502 & 9.2 & 400.0 \\
1.15 & 501 & 513 & 9.9 & 312.5 \\
1.09 & 498 & 457 & 9.9 & 247.5 \\
1.03 & 500 & 479 & 14.5 & 155.0 \\
0.98 & 444 & 475 & 2.3 & 96.5 \\
0.98 & 514 & 479 & 9.5 & 97.5 \\
0.93 & 551 & 483 & 42.6 & 51.0 \\
0.88 & 469 & 457 & 62.3 & 31.0 \\
0.84 & 421 & 463 & 213.6 & 20.5 \\
0.80 & 402 & 482 & 312.9 & 16.5 \\
0.76 & 384 & 459 & 527.3 & 14.0 \\
0.72 & 349 & 422 & 667.6 & 9.0 \\
\hline
\end{tabular}


Data for Figures 5.3, 5.4 \& 5.5

Acidifying slurries prepared at differing $\mathrm{pHs}$ and with different $\mathrm{Ca} / \mathrm{Si}$ ratios

Reaction conditions

Total volume: $0.5 \mathrm{~L}$

$\left[\mathrm{SiO}_{2}\right]_{\mathrm{m}}$

$0.50 \mathrm{~mol} / \mathrm{L}$

[Ca]

$0.62 \mathrm{~mol} / \mathrm{L}$

$[\mathrm{HCl}] \quad$ listed for each series

Initial acid:

$0.21 \mathrm{~mol} / \mathrm{L}$

$10 \mathrm{~mL}$

\begin{tabular}{cc}
\hline $\mathrm{pH}$ & $\begin{array}{c}\mathrm{OA} \\
(\mathrm{g} / 100 \mathrm{~g})\end{array}$ \\
\hline 12.8 & 79 \\
12.0 & 80 \\
11.0 & 210 \\
10.5 & 238 \\
10.0 & 230 \\
\hline
\end{tabular}

Initial acid:

$0.53 \mathrm{~mol} / \mathrm{L}$

$25 \mathrm{~mL}$

OA

\begin{tabular}{cc}
$\mathrm{pH}$ & $(\mathrm{g} / 100 \mathrm{~g})$ \\
\hline 10.9 & 152
\end{tabular}

$10.5 \quad 162$

$10.0 \quad 179$

$9.5 \quad 185$

184
Initial acid:

$0.32 \mathrm{~mol} / \mathrm{L}$

$15 \mathrm{~mL}$

\begin{tabular}{cc}
\hline & OA \\
$\mathrm{pH}$ & $(\mathrm{g} / 100 \mathrm{~g})$ \\
\hline 12.5 & 83
\end{tabular}

$12.0 \quad 107$

$11.0 \quad 266$

$9.9 \quad 298$

$9.0 \quad 237$

Initial acid:

0.63

30

$\mathrm{mL}$

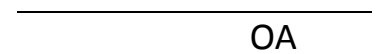

\begin{tabular}{cc}
$\mathrm{pH}$ & $(\mathrm{g} / 100 \mathrm{~g})$ \\
\hline 10.6 & 146
\end{tabular}

$10.3 \quad 144$

$10.0 \quad 153$

$9.5 \quad 157$

$9.0 \quad 145$
Initial acid:

$0.42 \mathrm{~mol} / \mathrm{L}$

$20 \mathrm{~mL}$

\begin{tabular}{cc}
\hline & OA \\
$\mathrm{pH}$ & $(\mathrm{g} / 100 \mathrm{~g})$ \\
\hline 12.0 & 123 \\
11.0 & 253 \\
10.5 & 258 \\
10.0 & 249 \\
9.5 & 255 \\
\hline
\end{tabular}

Initial acid:

$0.74 \mathrm{~mol} / \mathrm{L}$

$35 \mathrm{~mL}$

\begin{tabular}{cc}
\hline & OA \\
$\mathrm{pH}$ & $(\mathrm{g} / 100 \mathrm{~g})$ \\
\hline 10.4 & 139 \\
10.0 & 141 \\
9.5 & 131 \\
9.0 & 142 \\
\end{tabular}


Reaction conditions

Total volume: $0.5 \mathrm{~L}$

$\left[\mathrm{SiO}_{2}\right]_{\mathrm{m}} \quad 0.50 \quad \mathrm{~mol} / \mathrm{L}$

[Ca] $\quad 0.50 \quad \mathrm{~mol} / \mathrm{L}$

$[\mathrm{HCl}]$ listed for each series

Initial acid:

$0.21 \mathrm{~mol} / \mathrm{L}$

$10 \mathrm{~mL}$

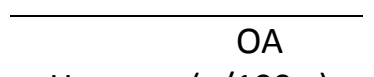

$\mathrm{pH} \quad(\mathrm{g} / 100 \mathrm{~g})$

$12.9 \quad 114$

$12.0 \quad 183$

$11.0 \quad 236$

$10.5 \quad 229$

$10.0 \quad 228$

Initial acid:

$0.53 \mathrm{~mol} / \mathrm{L}$

$25 \mathrm{~mL}$

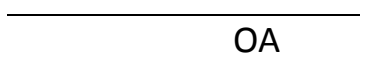

\begin{tabular}{cc}
$\mathrm{pH}$ & $(\mathrm{g} / 100 \mathrm{~g})$ \\
\hline 10.9 & 151
\end{tabular}

$10.5 \quad 148$

$10.0 \quad 140$

$9.5 \quad 152$

$9.0 \quad 149$
Initial acid: Initial acid:

$\begin{array}{rlrl}0.32 & \mathrm{~mol} / \mathrm{L} & 0.42 & \mathrm{~mol} / \mathrm{L} \\ 15 & \mathrm{~mL} & 20 \mathrm{~mL}\end{array}$
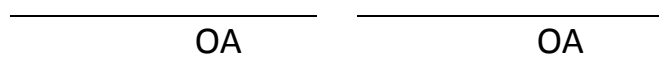

\begin{tabular}{lcllc}
$\mathrm{pH}$ & $(\mathrm{g} / 100 \mathrm{~g})$ & & $\mathrm{pH}$ & $(\mathrm{g} / 100 \mathrm{~g})$ \\
\cline { 1 - 2 } \cline { 5 - 6 } 12.2 & 167 & & 11.4 & 170
\end{tabular}

$\begin{array}{llll}11.0 & 227 & 11.0 & 199\end{array}$

$\begin{array}{llll}10.5 & 249 & 10.5 & 200\end{array}$

$\begin{array}{llll}10.0 & 236 & 10.0 & 207\end{array}$

$9.5 \quad 218$

$9.5 \quad 195$

Initial acid:

$0.63 \mathrm{~mol} / \mathrm{L}$

$30 \mathrm{~mL}$

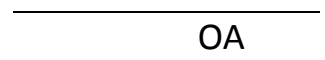

\begin{tabular}{lc}
$\mathrm{pH}$ & $(\mathrm{g} / 100 \mathrm{~g})$ \\
\hline 10.6 & 162
\end{tabular}

$10.0 \quad 153$

$9.5 \quad 150$

$9.0 \quad 139$ 
Reaction conditions

Total volume: $0.5 \mathrm{~L}$

$\left[\mathrm{SiO}_{2}\right]_{\mathrm{m}} \quad 0.50 \quad \mathrm{~mol} / \mathrm{L}$

[Ca] $\quad 0.75 \mathrm{~mol} / \mathrm{L}$

$[\mathrm{HCl}]$ listed for each series

Initial acid:

$0.21 \mathrm{~mol} / \mathrm{L}$

$10 \mathrm{~mL}$

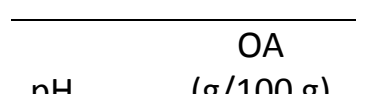

\begin{tabular}{cc}
$\mathrm{pH}$ & $(\mathrm{g} / 100 \mathrm{~g})$ \\
\hline 13.8 & 80
\end{tabular}

$13.0 \quad 101$

$12.0 \quad 110$

$11.0 \quad 230$

$10.0 \quad 224$

$9.0 \quad 207$

Initial acid:

$0.21 \mathrm{~mol} / \mathrm{L}$

$10 \mathrm{~mL}$

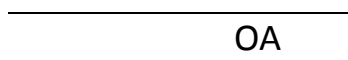

\begin{tabular}{cc}
$\mathrm{pH}$ & $(\mathrm{g} / 100 \mathrm{~g})$ \\
\hline 12.5 & 87
\end{tabular}

$12.0 \quad 108$

$11.0 \quad 211$

$10.5 \quad 252$

$10.0 \quad 242$
Initial acid:

$0.32 \mathrm{~mol} / \mathrm{L}$

$15 \mathrm{~mL}$

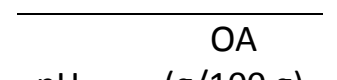

\begin{tabular}{cc}
$\mathrm{pH}$ & $(\mathrm{g} / 100 \mathrm{~g})$ \\
\hline 12.8 & 94
\end{tabular}

$12.0 \quad 120$

$11.0 \quad 249$

10.5

10.0

253

272

72

Initial acid:

$0.32 \mathrm{~mol} / \mathrm{L}$

$15 \mathrm{~mL}$

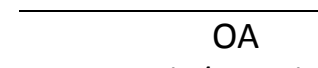

\begin{tabular}{cc}
$\mathrm{pH}$ & $(\mathrm{g} / 100 \mathrm{~g})$ \\
\hline 11.5 & 116
\end{tabular}

$11.0 \quad 196$

10.5

$10.0 \quad 252$

$9.2 \quad 244$

Initial acid:

$0.42 \mathrm{~mol} / \mathrm{L}$

$20 \mathrm{~mL}$

\begin{tabular}{lc}
\hline & $\mathrm{OA}$ \\
$\mathrm{pH}$ & $(\mathrm{g} / 100 \mathrm{~g})$ \\
\hline 12.7 & 79 \\
11.7 & 114 \\
11.0 & 213 \\
10.3 & 263 \\
10.0 & 272 \\
\hline
\end{tabular}

Initial acid:

$0.42 \mathrm{~mol} / \mathrm{L}$

$20 \mathrm{~mL}$

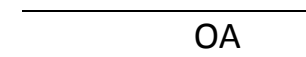

\begin{tabular}{lc}
$\mathrm{pH}$ & $(\mathrm{g} / 100 \mathrm{~g})$ \\
\hline 11.0 & 160
\end{tabular}

$10.5 \quad 186$

$10.0 \quad 208$

$9.5 \quad 201$

$9.0 \quad 195$


Data for Figures 5.9, 5.10, \& 5.11

Effect of altering calcium and hydroxide concentrations.

Reaction conditions

Total volume: $0.25 \mathrm{~L}$

$\left[\mathrm{SiO}_{2}\right]_{\mathrm{m}} \quad 0.58 \quad \mathrm{~mol} / \mathrm{L}$

Oil absorptions of 2-ethoxyethanol washed samples (g/100 g)

$\left[\mathrm{OH}^{-}\right](\mathrm{mol} / \mathrm{L})$

\begin{tabular}{l|llll} 
& 0.94 & 0.70 & 0.47 & 0.23 \\
\hline 0.62 & 429 & 367 & 151 & 149
\end{tabular}

$\begin{array}{llllll}{[\mathrm{Ca}]} & 0.47 & 427 & 198 & 140 & 166\end{array}$

$\begin{array}{llllll}(\mathrm{mol} / \mathrm{L}) & 0.31 & 240 & 174 & 164 & 156\end{array}$

$\begin{array}{lllll}0.16 & 246 & 215 & 205 & 216\end{array}$

Oil absorptions of water washed samples (g/100 g)

\begin{tabular}{lc|cccc} 
& & \multicolumn{4}{|c}{$\left[\mathrm{OH}^{-}\right](\mathrm{mol} / \mathrm{L})$} \\
& & 0.94 & 0.70 & 0.47 & 0.23 \\
\cline { 2 - 6 }$[\mathrm{Ca}]$ & 0.62 & 104 & 100 & 155 & 150 \\
$(\mathrm{~mol} / \mathrm{L})$ & 0.47 & 44 & 45 & 139 & 145 \\
& 0.31 & 41 & 57 & 151 & 142 \\
& 0.16 & 27 & 63 & 126 & 136
\end{tabular}

Calculated masses of total product (g)

\begin{tabular}{lc|cccc} 
& & \multicolumn{4}{|c}{$\left[\mathrm{OH}^{-}\right](\mathrm{mol} / \mathrm{L})$} \\
& & 0.94 & 0.70 & 0.47 & 0.23 \\
\cline { 2 - 6 }$[\mathrm{Ca}]$ & 0.62 & 19.46 & 16.7 & 15.33 & 13.55 \\
$(\mathrm{~mol} / \mathrm{L})$ & 0.47 & 17.12 & 17.21 & 15.92 & 14.96 \\
& 0.31 & 12.99 & 13.9 & 14.03 & 13.98 \\
& 0.16 & 6.75 & 7.18 & 8.21 & 10.74
\end{tabular}




\section{Data for Figures 5.12 \& 5.13}

Effect of temperature and mixing

Reaction conditions

Total volume: $1 \mathrm{~L}$

$\begin{array}{lll}{\left[\mathrm{SiO}_{2}\right]_{\mathrm{m}}} & 0.165 & \mathrm{~mol} / \mathrm{L} \\ {[\mathrm{Ca}]} & 0.156 & \mathrm{~mol} / \mathrm{L} \\ {[\mathrm{HCl}]} & 0.079 & \mathrm{~mol} / \mathrm{L}\end{array}$

\begin{tabular}{|c|c|c|c|c|c|}
\hline $\begin{array}{l}\text { Time } \\
\text { (min) }\end{array}$ & $\begin{array}{c}\text { OA } \\
(\mathrm{g} / 100 \\
\mathrm{g}) \\
\end{array}$ & $\begin{array}{l}\text { Time } \\
(\min )\end{array}$ & $\begin{array}{c}\text { OA } \\
(\mathrm{g} / 100 \\
\mathrm{g})\end{array}$ & $\begin{array}{l}\text { Time } \\
\text { (min) }\end{array}$ & $\begin{array}{c}\mathrm{OA} \\
(\mathrm{g} / 100 \\
\mathrm{g})\end{array}$ \\
\hline 2 & 154.5 & 2 & 134 & 2 & 153.5 \\
\hline 5 & 163 & 5 & 128 & 5 & 170 \\
\hline 10 & 186.5 & 10 & 142 & 10 & 190 \\
\hline 30 & 270.5 & 30 & 192 & 30 & 275.5 \\
\hline 60 & 297.5 & 60 & 256 & 60 & 330 \\
\hline 150 & 466 & 150 & 388 & 150 & 502 \\
\hline 284 & 596.5 & 300 & 492 & 300 & 595 \\
\hline
\end{tabular}

Data for Figure 5.14

Effect of overall reaction concentration.

Reaction conditions

$\mathrm{Ca} / \mathrm{Si} \quad 0.96$

\begin{tabular}{cc}
\hline$\left[\mathrm{SiO}_{2}\right]_{\mathrm{m}}$ & $\mathrm{OA}$ \\
$(\mathrm{mg} / \mathrm{L})$ & $(\mathrm{g} / 100 \mathrm{~g})$ \\
\hline 0.662 & 410 \\
0.473 & 465 \\
0.414 & 500 \\
0.331 & 510 \\
0.165 & 510 \\
0.082 & 520 \\
0.082 & 530 \\
\hline
\end{tabular}




\section{Appendix B - Supplemental geothermal}

\section{sample data}

Optical properties of samples prepared at Kawerau and Rotokawa during first visit.

\begin{tabular}{|l|ccc|cc|}
\hline Sample & D65 & & & Reflectance & Reflectance \\
L & $\mathrm{a}^{*}$ & $\mathrm{~b}^{*}$ & $457 \mathrm{~nm}$ & $580 \mathrm{~nm}$ \\
\hline K1 & 96.23 & 0.34 & 2.26 & 87.49 & 91.51 \\
K2 & 97.01 & 0.38 & 1.61 & 90.14 & 93.25 \\
K3 & 96.76 & 0.29 & 1.89 & 89.19 & 92.68 \\
K5 & 96.63 & 0.20 & 2.24 & 88.47 & 92.42 \\
K6 & 96.61 & 0.25 & 2.02 & 88.68 & 92.34 \\
K7 & 96.94 & 0.21 & 2.34 & 89.03 & 93.23 \\
K8 & 96.96 & 0.18 & 1.64 & 90.00 & 93.07 \\
K9 & 96.52 & 0.15 & 1.89 & 88.63 & 92.03 \\
K10 & 96.99 & 0.13 & 1.68 & 90.03 & 93.13 \\
K11 & 97.11 & 0.14 & 1.44 & 90.61 & 93.36 \\
K12 & 96.93 & 0.13 & 1.70 & 89.86 & 92.99 \\
K13 & 97.17 & 0.10 & 1.35 & 90.88 & 93.45 \\
K14 & 97.11 & 0.13 & 1.44 & 90.62 & 93.34 \\
K15 & 97.28 & 0.15 & 1.13 & 91.39 & 93.67 \\
K16 & 97.29 & 0.13 & 1.12 & 91.46 & 93.68 \\
K17 & 97.10 & 0.09 & 1.60 & 90.40 & 93.35 \\
K18 & 97.27 & 0.13 & 1.27 & 91.22 & 93.69 \\
K19 & 97.18 & 0.12 & 1.24 & 91.04 & 93.46 \\
K20 & 97.51 & 0.15 & 0.81 & 92.39 & 94.13 \\
K21 & 97.07 & 0.12 & 1.44 & 90.51 & 93.21 \\
K22 & 97.32 & 0.11 & 1.28 & 91.32 & 93.79 \\
K23 & 96.91 & 0.14 & 1.29 & 90.32 & 92.79 \\
K24 & 97.07 & 0.13 & 1.11 & 90.95 & 93.14 \\
K25 & 96.83 & 0.10 & 1.36 & 90.03 & 92.60 \\
K26 & 97.22 & 0.11 & 1.28 & 91.13 & 93.54 \\
K27 & 97.14 & 0.09 & 1.25 & 90.94 & 93.36 \\
K28 & 96.99 & 0.15 & 1.31 & 90.50 & 92.98 \\
K29 & 97.58 & 0.14 & 0.72 & 92.69 & 94.27 \\
K30 & 96.95 & 0.13 & 1.43 & 90.22 & 92.92 \\
K31 & 96.89 & 0.16 & 1.34 & 90.21 & 92.80 \\
R1 & 96.85 & 0.41 & 1.46 & 89.94 & 92.87 \\
R2 & 96.92 & 0.38 & 1.48 & 90.13 & 93.00 \\
R3 & 97.35 & 0.25 & 1.43 & 91.23 & 94.00 \\
R4 & 96.63 & 0.22 & 1.87 & 88.95 & 92.34 \\
R5 & 96.45 & 0.22 & 2.06 & 88.27 & 91.94 \\
R6 & 96.46 & 0.19 & 2.04 & 88.33 & 91.96 \\
R7 & 97.38 & 0.23 & 1.50 & 91.22 & 94.06 \\
& 97.19 & 0.27 & 1.20 & 91.13 & 93.54 \\
\hline
\end{tabular}




\section{Appendix C - Patent}

Johnston, J.H., Borrmann,T. \& McFarlane, A.J. (2006). Nano-structured Silicate, Functionalised Forms Thereof, Preparation and Uses. NZ Patent Specification No. 537747.; International PCT Application PCT/NZ2006/000003.

The contribution of this author to the patent was the centred upon the development and preparation of the nano-structured calcium silicate as can be found in preparations 1 to 7 and examples thereof numbered 1 to 4 . Studies into the use of the material for passive humidity control (application example 6) were performed by the author. Materials were also provided by the author to collaborators for testing as a paper filler, as seen an application examples 1 and 2. Data as measured by the author can be seen in Figures 2, 3, 5, 6, 7, 8, and 11. 


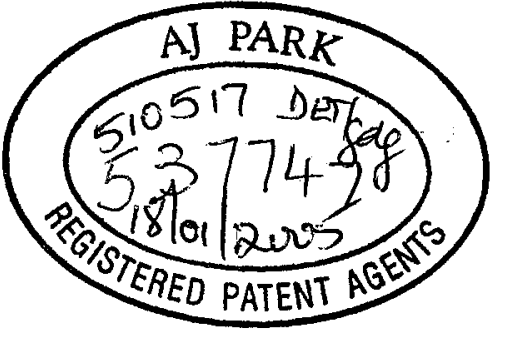

NEW ZEALAND

PATENTS ACT, 1953

\section{PROVISIONAL SPECIFICATION}

NANO-STRUCTURED SILICATE, FUNCTIONALISED FORMS THEREOF, PREPARATION AND USES

We, VICTORIA LINK LIMITED, a New Zealand company of 15 Mount Street, Kelburn, Wellington, New Zealand, do hereby declare this invention to be described in the following statement: 
Nano-Structured Silicate, Functionalised Forms Thereof, Preparation and Uses

This invention relates to the preparation, functionalisation and use of a novel nano-structured silicate generally calcium silicates which may be hydrated. It also relates to novel methods of producing nano-structured silicates.

\section{Background Art}

Silicas comprising submicron particles arranged in various microstructural forms, notably essentially individual particles (fume silicas), networks (precipitated silicas or silicates) and random close packed structures (gels) are well known and are widely used in many different industry and consumer applications. These materials are well characterised and their various methods of preparation, structures, properties and applications are presented in standard texts such as Iler (1973) (Ralph K. Iler - The Chemistry of Silica, WileyInterscience, New York, 1979) and numerous research publications, patents and information and applications sheets by commercial suppliers. Sodium silicate (water glass) is generally used as the starting material for the preparation of precipitated silicas (and silicates) and silica gels in an aqueous system wherein the structural type, characteristics and properties of the particular silica (silicate) or gel are generally determined by control of concentration of the sodium silicate solution and the reduction in $\mathrm{pH}$ (usually by the addition of sulfuric acid) to initiate precipitation or gel formation followed by a similar control of the mixing, ageing and drying process. The details of many of these preparations are proprietary to commercial manufacturers.

Precipitated silicas with a network structure have also been produced from geothermal water which contains much lower levels of dissolved silica, typically up to about 1000 mg.kg ${ }^{-1} \mathrm{SiO}_{2}$ with the product being successfully tested as a filler in newsprint to reduce print-through and enhance print quality (Harper and Johnston, 1997) (US Patent No. $5,595,717)$. 
An aqueous solution of sodium silicate is highly alkaline with a $\mathrm{pH}$ typically greater than about $\mathrm{pH}=12$, depending upon the dissolved silica concentration. The dominant species here are the $\mathrm{H}_{3} \mathrm{SiO}_{4}{ }^{-}$ion and the $\mathrm{H}_{2} \mathrm{SiO}_{4}{ }^{2-}$ ion. The addition of acid reduces the $\mathrm{pH}$ which initiates polymerisation of these ions to produce oxygen bridged silicate polymers which can be represented simply as:

$$
\begin{aligned}
& (\mathrm{HO})_{3} \mathrm{SiO}^{-}+{ }^{-} \mathrm{OSi}(\mathrm{OH})_{3} \rightarrow(\mathrm{HO})_{3} \operatorname{SiOSi}(\mathrm{OH})_{2} \mathrm{O}^{-}+\mathrm{H}_{2} \mathrm{O} \\
& (\mathrm{HO})_{3} \mathrm{SiOSi}(\mathrm{OH})_{2} \mathrm{O}^{-}+\mathrm{n}\left[{ }^{-} \mathrm{OSi}(\mathrm{OH})_{3}\right] \rightarrow\left[(\mathrm{HO})_{3} \mathrm{SiOSi}(\mathrm{OH})_{2} \mathrm{O}^{-}\right]_{\mathrm{n}}+\mathrm{nH}_{2} \mathrm{O} \\
& \text { Colloidal silica particle }
\end{aligned}
$$

This polymerisation takes place in 3-dimensions to form nano-sized spherical silica particles which then form the requisite network or gel structures. The surfaces of the particles usually have a high density of silanol groups.

The process for precipitating silica from geothermal water which is supersaturated with dissolved silica, as described by Harper and Johnston (1997) (US Patent No. 5,595,717, 1997), involves allowing the water to age at a $\mathrm{pH}$ of about $\mathrm{pH}=7-9$, whereupon nano-size colloidal particles of silica form according to the above mechanism. Because of the surface silanol groups these particles have a negative surface charge and will form a colloid. The addition of a metal cation, typically $\mathrm{Ca}^{2+}$ neutralizes this surface charge to some extent and allows these particles to come together to form a precipitated silica with a network structure. The size of the particles and hence the strength of the network structure may be reinforced by exposing the precipitate to geothermal water containing unpolymerised dissolved silica $\left(\mathrm{H}_{3} \mathrm{SiO}_{4}{ }^{-}\right)$and recovering this silica on the network structure of the original precipitated silica (Harper and Johnston, 1997) (US Patent No. 5,595,717, 1997). 


\section{Summary of the Invention}

In accordance with the invention there is provided a nano-structured calcium silicate material which comprises nano-size platelets about 5-10 nm thick and about 50-200 nm wide stacked together in a poorly-ordered open framework type structure to provide pores which are accessible.

The platelets have a high surface area which is also accessible. The platelets are generally not planar and have a complex curved morphology similar to a rose petal. They are X-ray amorphous and have no long range order.

A nano-structure is one which normally has one dimension less than $100 \mathrm{~nm}$. By a nano-structure in accordance with the invention, platelets are present, which are of a 5-10 nm thickness and a plate surface width of 50 to $200 \mathrm{~nm}$.

These platelets are stacked in a poorly-ordered open framework type structure to yield particles. These particles vary in size up to about 20 microns.

The platelets are stacked in such a way as to create the pores of the invention and the high surface area, in the nano-structured calcium silicate material which are accessible. By accessibility, is meant that the openings of the pores are quite large in relation to the volume of the interior of the pore. The diameter of the pore opening approximates to the width of the platelets (Figure 1). A range of various agents and compounds can readily enter and be accommodated within the pores and/or on the surface of the platelets defining the pore. This contrasts with a material that may have a similar pore volume but a much narrower pore opening, somewhat like a bottle, or a zeolite type material that has both a much smaller pore volume and pore opening. Such smaller pores and pores with a narrower opening are accessible only to small molecules. The larger volume and the wider opening of the pores in the material of this invention mean these pores are accessible to a wide range of other agents and compounds, particularly bulky ones. The surfaces of the platelets defining the pores are also readily accessible and can bind such other agents or compounds. 
The invention also provides a nano-structured calcium silicate material as defined above where the calcium is partially or substantially completely replaced by hydrogen ions by acid washing the calcium silicate at a $\mathrm{pH}$ above about 6 .

The invention further provides such a calcium silicate as defined above reinforced by a monomeric silicate material.

The invention further provides such nano-structured silicate materials as defined above which are hydrated wherein the water molecules are hydrogen bonded to the $\mathrm{Ca}^{2+}$ ions at coordination sites on the $\mathrm{Ca}^{2+}$ ions that are not associated with bonding to the surface silanol (-Si-OH) groups on the platelet surfaces. Water molecules can also hydrogen bond directly to such surface silanol groups not associated with the $\mathrm{Ca}^{2+}$ ions.

The invention further provides a silicate material as defined above in which the oil absorption is greater than $350 \mathrm{~g}$. oil. $100 \mathrm{~g}^{-1}$ silicate.

The invention further provides a silicate material as defined above in which the oil absorption is greater than $400 \mathrm{~g}$. oil. $100 \mathrm{~g}^{-1}$ silicate.

The invention further provides a silicate material as defined above in which the oil absorption is greater than $500 \mathrm{~g}$. 0 il. $100 \mathrm{~g}^{-1}$ silicate.

The invention further provides a silicate material as defined above in which water is replaced by a spacer compound.

The invention further provides a silicate material as defined above in which the spacer compound has hydrogen-bonding capacity and/or a higher boiling point than water.

The invention further provides a silicate material as defined above, in which the nano-structure has been reinforced by addition of further silica or silicate to the structure. 
The invention further provides a silicate material as defined above, in which cations, anions and neutral molecules are accommodated in the pores and/or on the surface of the platelets in the nano-structure.

The invention also provides a novel nano-structured silicate material prepared by reacting a calcium ion containing solution or slurry with a silicate containing solution, allowing the calcium silicate to precipitate and ageing that product to increase the order of the nano-structure, oil absorption and surface area characteristics, optionally acid washing the material, optionally reinforcing the material, optionally replacing any water within the structure with a spacer compound and optionally drying the material, and optionally accommodating anions and neutral molecules in the pores and/or on the surface of the platelets.

The invention further provides in the process of preparation the following optional conditions:

i. where the $\mathrm{pH}$ of the calcium and silicate solutions/slurries are matched;

ii. where the $\mathrm{Ca}^{++}$is added in an excess molar amount (preferably 5-10\%) in comparison to the Si present;

iii. where the addition of the calcium to the silicate solution is rapid;

iv. where the rapid addition is accompanied by vigorous stirring;

v. where the ageing process happens with additional gentle stirring;

vi. where the ageing process happens on standing;

vii. where water is removed by drying;

viii. where water is substituted by a spacer compound;

ix. where that spacer compound is 2-ethoxyethanol (2-EE) or 2-methoxyethanol (2-ME);

x. where the spacer compound is added by plug washing of the filter cake;

xi. where the calcium silicate precipitate is strengthened by addition of further monomeric silicate material;

xii. where the strengthening is through adding a sodium silicate solution. 
The invention further provides that the silicate material of the invention is then functionalised in all the various forms;

a. By incorporating phase change material for heat storage and release applications.

b. By incorporating iodine, sulfur; metals and their cations for example copper, zinc, silver; and organic molecules for example omacide and hexanal for anti-microbial and biocidal applications.

c. By incorporating metal oxy-anions for example vanadate ions; and metal ions for example zinc, copper for anti-corrosion applications;

d. By incorporating essential oils, perfumes, aroma and odouriferous compounds including foul-smelling compounds, for the controlled release of such aromas and odours.

e. By incorporating photoactive centres for example titanium dioxide for photoactive and photochemical applications.

f. By incorporating cations for example copper, anions for example phosphate or neutral molecules for example iodine for the transport and/or slow release of these species.

g. By incorporating conducting polymers for example polyaniline, polypyrrole and polythiophene, and their various derivatives to provide oxidation-reduction properties, electronic conductivity, opto-electronic properties, anti-corrosive and anti-microbial properties.

h. By incorporation of ionic conducting materials for solid electrolyte applications.

i. By incorporating metal and/or metal oxide nanoparticles.

j. By incorporating magnetic centres or metals of metal oxides.

k. By incorporating metal or metal ion centres for example rhodium for catalytic purposes. 
The invention also provides a calcium or other silicate material produced by one or more of the methods of the invention. The processes of the invention produce novel silicate materials having high oil absorption capacity and surface area and consisting of nano-sized platelets.

\section{Brief reference to the Drawings}

The invention will be described with reference to the attached drawings in which:

Figure 1 shows electronmicroscope photographs of a nano-structured calcium silicate of the invention, depicting the open framework of nano-size platelets that provide the accessible large pore volume and accessible large surface area.

Figure 2 is a graph showing the variation on the oil absorption and hence the development of the nano-structure of a calcium silicate of the invention in relation to concentration of dissolved silica.

Figure 3 shows the effect of varying the mole fraction of calcium ions and hydroxyl ions on the properties of the calcium silicate of the invention.

Figure 4 is a series of electronmicroscope images showing the development of the nano-structure of a calcium silicate of the invention during ageing. The left-hand photograph is at 10 minutes, the central photograph at 60 minutes and the right-hand photograph is at 360 minutes of ageing time.

Figure 5 shows the effect of ageing time on the properties of the calcium silicate of the invention.

Figure 6 shows the effect of stirring and vessel size on the properties of the calcium silicate of the invention. 
Figure 7 shows the effect of washing of the calcium silicate of the invention with 2-ethoxyethanol.

Figure 8 shows the effect of reinforcing the calcium silicate material of the invention with monomeric silica.

Figure 9 are photographs showing the anticorrosive performance of a nano-structured calcium silicate vanadate of this invention.

Figure 10 shows the performance of a nano-structured calcium silicate of this invention as a filler in newsprint.

Figure 11 shows the uptake and release of water vapour of a nano-structured calcium silicate of this invention.

Figure 12 shows the effect of nano-structured calcium silicate on ripening of fruit.

Figure 13 shows the antimicrobial action of nano-structured calcium silicate composites of the invention with iodine and sulphur.

\section{Detailed Description of the Invention}

The novel nano-structured silicate material generally comprises particles of up to 20 microns in size with each particle itself comprising nano-size platelets about 5-10 nm thick and up about 50-200 nm wide stacked together in a poorly ordered open framework type structure, forming a silicate sponge. This somewhat resembles the petals of an open rose flower - hence is termed a "desert rose" type structure. This bestows onto the material the desirable properties of a high accessible pore volume and liquid absorption capacity and a high accessible surface area. The surfaces of the platelets can be functionalized by adsorbing or bonding a variety of cations, anions and neutral molecules which provide materials with further novel or improved properties that can be utilized in a range of applications. 
The extent of openness of the framework structure and hence the magnitude of the pore volume and surface area and propensity for functionalisation can be controlled at least to some extent in the preparation of the material, particularly to reduce collapsing or partial collapsing of the structure to where the platelets stack in a more parallel type arrangement (somewhat resembling a closed rose flower).

The typical structure and morphology of the open framework "desert rose" structure showing particles of up to about 20 microns in size with each particle itself comprising nano-size platelets is shown in Figure 1.

The nano-structured calcium silicate material and its various functionalised forms have applications in at least the following areas:

- As a material with a high liquid absorbency for use in the absorption of liquids.

- As an inert carrier for liquids or vapours.

- As a slow release agent for liquids and vapours.

- Paper filling and paper coating to improve print and optical properties of the paper.

- In paper packaging to improve liquid and vapour absorbency and provide a controlled environment.

- In heat storage and release applications by incorporating relatively high levels of phase change energy storage and release materials.

- As a material for gas adsorption in humidity control and in the control of fruit ripening.

- As a material with a high surface area for use in catalysis, photoactivity and photochemistry, and a surface for chemical reactions.

- The (selective) adsorption or absorption of metal ions, anions and neutral species from liquids, solutions or gases.

- Anti-corrosive and anti-microbial applications, particularly in surface coatings.

- As a material for absorption of conducting polymers, nanoparticles, and other compounds with special electronic, electrochemical, magnetic and physical properties.

The preparation of this novel nano-structured calcium silicate material involves the direct addition of calcium ions to a solution of dissolved silica, usually sodium silicate, which is present mainly as $\mathrm{H}_{3} \mathrm{SiO}_{4}{ }^{-}$silicate ions and $\mathrm{H}_{2} \mathrm{SiO}_{4}{ }^{2-}$ ions, and possibly other species such 
as $\mathrm{H}_{4} \mathrm{SiO}_{4}$, under controlled conditions of $\mathrm{pH}$, mixing, temperature, ageing and post treatment as detailed below. The calcium ions and hydroxyl ions may be added as a solution or slurry of calcium hydroxide for which the $\mathrm{pH}$ may be adjusted with acid prior to addition. The reaction may be summarized as:

$$
\mathrm{H}_{3} \mathrm{SiO}_{4}^{-}+\mathrm{Ca}^{2+}+\mathrm{OH}^{-} \rightarrow \mathrm{CaSiO}_{\mathrm{x}}(\mathrm{OH})_{\mathrm{y}}+\mathrm{H}_{2} \mathrm{O}
$$

Where: $\quad \mathrm{x}$ is approximately $2-3$

$\mathrm{y}$ is approximately $1-2$

The reaction is preferably carried out at about room temperature $\left(15-25^{\circ} \mathrm{C}\right)$. The material is formed as a precipitate or as a slurry.

This precipitate or slurry may be subjected to post treatment such as functionalisation by the addition of appropriate anions, cations or neutral molecules, filtered, washed and dried as appropriate. Also, functionalisation can be achieved by adding appropriate anions, cations or neutral molecules to the solution of dissolved silica or sodium silicate solution.

The nano-structured calcium silicate material comprises particles of up to about 20 microns in size with each particle itself comprising nano-size platelets about 5-10 nm thick and up about 50-200 nm wide stacked together in a poorly ordered open framework type structure (somewhat resembling the petals of an open rose flower - "desert rose" type structure). The nano-structure develops during a carefully controlled ageing step following the initial precipitation of the calcium silicate material. As a consequence and depending upon the actual preparation method used, the material has the desirable properties of a high accessible pore volume and liquid absorption capacity. The liquid absorption capacity can be measured by the ASTM D281-31 (1980). Spatula Rub-Out method.

The oil absorption capacity of products of this invention even in the collapsed state, produced on drying the initially formed nano-structured material, is still generally above $100 \mathrm{~g}$.oil. $100 \mathrm{~g}^{-1}$ silicate material. The more preferred nano-structured materials in which the "desert rose" structure is more apparent, can have oil absorptions as high as 
$600 \mathrm{~g} .0 \mathrm{il} .100 \mathrm{~g}^{-1}$ silicate material. It is envisaged within this invention that even higher oil absorption capacity such as up to about $700 \mathrm{~g}$. oil.100 g. ${ }^{-1}$ silicate material can be achieved if so desired. There will invariably be a balance between the higher absorption capabilities and the consequent additional costs.

It is within the scope of the invention for the collapsed form of the nano-structured calcium silicate to be utilized in view of its oil absorption characteristics. For example in paper filling and coating techniques, it can improve print and optical properties of the paper.

The collapsed material can be regenerated to a certain extent by reslurrying in water and stirring but normally it is not possible to return the collapsed material to the absorption capacity of the original material.

Surface areas of the nano-structured products of the invention of up to about $600 \mathrm{~m}^{2} \cdot \mathrm{g}^{-1}$ can be obtained.

In addition, the surfaces of the nano-size platelets which initially contain mainly silanol groups and bound calcium ions which may be hydrated, enable the material to be functionalized by adsorbing or bonding a variety of cations, anions and neutral molecules onto these platelet surfaces. The extent of openness of the framework structure and hence the magnitude of the pore volume and surface area and propensity for or extent of functionalisation can be controlled to some extent in the preparation process. The pore volume and surface area can be reduced in the preparation process, particularly on drying, wherein a collapsed or partially collapsed type structure where the platelets stack in a more parallel type arrangement (somewhat resembling a closed rose flower) is formed. Accordingly, the oil absorption capacity can be reduced to about $100 \mathrm{~g} .011 .100 \mathrm{~g}^{-1}$ material, and the surface area reduced to about $100 \mathrm{~m}^{2} \cdot \mathrm{g}^{-1}$. In the drying process, the act of removing the occluded or pore water which is hydrogen bonded to the surface silanol groups, tends to pull the platelets together thereby partially collapsing the structure and reducing the pore volume and accessible surface area. 
In many applications of the nano-structured calcium silicate material both in the slurry form or dry state, it is desirable to utilize the high accessible pore volume and high accessible surface area. This can be achieved for material in the dry state by washing spacer compounds such as 2-ethoxyethanol or 2-methoxyethanol through the filtercake to displace the occluded or pore water before drying. In this way, oil absorptions of up to about $600 \mathrm{~g} .0 \mathrm{il} .100 \mathrm{~g}^{-1}$ material, and the surface areas of up to about $600 \mathrm{~m}^{2} \cdot \mathrm{g}^{-1}$ can be readily achieved. If this dried material is re-wetted then some of the spacer compound is replaced by water a partial collapse takes place and the oil absorption and surface area are reduced.

In the slurry and dry states, the high oil absorption and surface area of the material can be retained by reinforcing the nano-size platelets in the framework structure. This is achieved by adding a quantity of sodium silicate, or silicate containing solution to the slurry of nano-structured calcium silicate following precipitation and ageing. The dissolved silicate is recovered onto the surfaces, edges and corners of the nano-size platelets through polymerization with the surface silanol groups and reaction with the surface calcium ions, thereby strengthening the framework structure. Upon drying the pore volume and surface area are substantially retained with the dry material possessing oil absorptions up to about $400 \mathrm{~g} .0 \mathrm{il} .100 \mathrm{~g}^{-1}$ material, and the surface areas of up to about $400 \mathrm{~m}^{2} \cdot \mathrm{g}^{-1}$.

As the calcium ions bound to the surfaces of the platelets are also hydrated and act as centers for hydrogen bonding of occluded water, it has been discovered that the partial removal or removal of these calcium ions by washing the material with acid to reduce the $\mathrm{pH}$ of the material from its original alkaline value of about $\mathrm{pH}=10-11$ to about $\mathrm{pH}=6-9$, is also effective in preventing the partial collapse of the framework structure upon drying. In this way the pore volume and surface area are similarly substantially retained, with the acid washed and dried material possessing oil absorptions up to about $400 \mathrm{~g} .0 \mathrm{il} .100 \mathrm{~g}^{-1}$ material, and the surface areas of up to about $400 \mathrm{~m}^{2} \cdot \mathrm{g}^{-1}$.

In the process of acid washing, the calcium content can be progressively reduced depending upon the final $\mathrm{pH}$ of the acid washed material, and is less than about $1 \mathrm{wt} \% \mathrm{Ca}^{2+}$ at about $\mathrm{pH}=6$. 
The open nature of the framework structure afforded by the stacked arrangement of nano-size platelets, the accessible pore volume and large accessible surface area provides a large surface for functionalising by anions, cations and neutral molecules, for specific chemical reactions to take place, and for the absorption/adsorption and desorption of particular liquid and gaseous species.

\section{Preparation}

The preparation of nano-structured calcium silicate with different accessible pore volumes and surface areas, and in its various functionalised forms comprises the following steps:

\section{Preparation of a solution containing dissolved silica.}

This is generally in the form of the $\mathrm{H}_{3} \mathrm{SiO}_{4}{ }^{-}$silicate ions, but possibly also with $\mathrm{H}_{2} \mathrm{SiO}_{4}{ }^{2-}$ silicate ions and silicic acid $\mathrm{H}_{4} \mathrm{SiO}_{4}$ present. This includes sodium silicate solutions and geothermal water and other naturally occurring waters containing dissolved silica.

Where sodium silicate is used as the source of dissolved silica then solutions with concentrations of dissolved silica up to about $35,000 \mathrm{mg} \cdot \mathrm{kg}^{-1} \mathrm{SiO}_{2}$ can be readily used. This results in "thick" slurries of nano-structured calcium silicate that are still workable. Although nano-structured calcium silicate can be readily formed from sodium silicate or similar solutions with higher concentrations of dissolved silica, the increased thickness of the resulting slurry may present workability problems further on in the overall process.

The issue of workability is important in the ensuing ageing process (as discussed below) wherein the nano-structure is developed. It has been found that there is a balance between the concentration of the dissolved silica content in the starting solution, which essentially determines the thickness of the slurry of precipitated nano-structured calcium silicate, and the development of a nano-structure that exhibits a high accessible pore volume (oil absorption) and high accessible surface area in the ageing process. The optimum is when the dissolved silica concentration is about $7,000-10,000 \mathrm{mg} \cdot \mathrm{kg}^{-1} \mathrm{SiO}_{2}$. This provides an easily worked solution. 
Figure 2 shows the effect of the concentration of the dissolved silica solution on the development of the nano-structure during the ageing process (as discussed further below), as measured by the oil absorption on the dry nano-structured calcium silicate material treated with 2-ethoxyethanol (2-EE) to maintain the integrity of the nano-structure. The base concentration (dilution factor $=1$ ) is $35,000 \mathrm{mg} \cdot \mathrm{kg}^{-1} \mathrm{SiO}_{2}$. This shows the nano-structure and consequent pore volume and surface area are fully developed for dissolved silica concentrations of about $17,000 \mathrm{mg} \cdot \mathrm{kg}^{-1} \mathrm{SiO}_{2}$ (dilution factor approximately greater than 2).

The nano-structured calcium silicate can however be formed over a very wide range of concentrations from above $35,000 \mathrm{mg} \cdot \mathrm{kg}^{-1} \mathrm{SiO}_{2}$ to less than about $100 \mathrm{mg} \cdot \mathrm{kg}^{-1} \mathrm{SiO}_{2}$. For geothermal water the concentration of dissolved silica is typically up to about $1,000 \mathrm{mg} \cdot \mathrm{kg}^{-1} \mathrm{SiO}_{2}$.

It is preferable that this solution is prepared and maintained at room temperature (approximately $15-25^{\circ} \mathrm{C}$ ) to minimize energy costs. However it is recognized that temperatures up to $100^{\circ} \mathrm{C}$ or higher if the system is pressurized, can be used.

\section{Adding a solution or source of dissolved $\mathrm{Ca}^{2+}$ ions:}

\section{(a) at an appropriate ratio of $\mathrm{Ca}$ to $\mathrm{Si}$}

The addition of cations, usually $\mathrm{Ca}^{2+}$ ions to the above solution of dissolved silica precipitates a calcium silicate (hydrated) material with an early stage nano-structure. On a stoichiometric basis for a $\mathrm{CaSiO}_{3}$ type material, the $\mathrm{Ca}: \mathrm{Si}$ mole ratio should be ideally be 1:1. However, for the nano-structured calcium silicate material it has been found that a mole ratio of $\mathrm{Ca}^{2+}$ and dissolved $\mathrm{SiO}_{2}$ in solution which gives a slight excess of $\mathrm{Ca}^{2+}$ over dissolved $\mathrm{Si}$ is preferred, typically about $5-10 \%$ excess of $\mathrm{Ca}^{2+}$, as this ensures the rapid and complete precipitation of the calcium silicate. For example, this means that for a dissolved silica solution containing about $35,000 \mathrm{mg} \cdot \mathrm{kg}^{-1} \mathrm{SiO}_{2}$, the amount of dissolved $\mathrm{Ca}^{2+}$ added is about $25,000 \mathrm{mg} \cdot \mathrm{kg}^{-1} \mathrm{Ca}^{2+}$, and for a dissolved silica solution containing about 8,700 mg. $\mathrm{kg}^{-1} \mathrm{SiO}_{2}$, the amount of dissolved $\mathrm{Ca}^{2+}$ added is about $6,250 \mathrm{mg} \cdot \mathrm{kg}^{-1} \mathrm{Ca}^{2+}$. 


\section{(b) at an appropriate $\mathrm{pH}$}

The $\mathrm{pH}$ at which the addition of $\mathrm{Ca}^{2+}$ to the solution of dissolved silica is made is important. This should be approximately equal to that of the dissolved silica solution. For a dissolved silica solution prepared from sodium silicate and containing about $35,000 \mathrm{mg} \cdot \mathrm{kg}^{-1} \mathrm{SiO}_{2}$, the $\mathrm{pH}$ is about $\mathrm{pH}=11.5-11.7$. If the $\mathrm{Ca}^{2+}$ is added in the form of a solution of calcium chloride, the $\mathrm{pH}$ should be increased to approximately that of the sodium silicate solution by the addition of a base, such as sodium hydroxide to the $\mathrm{Ca}^{2+}$ containing solution before addition to the dissolved silica solution. A convenient way to add both $\mathrm{Ca}^{2+}$ and $\mathrm{OH}^{-}$ions is to utilize $\mathrm{Ca}(\mathrm{OH})_{2}$. However, since the solubility of $\mathrm{Ca}(\mathrm{OH})_{2}$ in water at about $1200 \mathrm{~g} \cdot \mathrm{kg}^{-1}$ at room temperature is significantly less than that generally required, the $\mathrm{Ca}(\mathrm{OH})_{2}$ is added as a slurry in water. Also, as the alkalinity of the $\mathrm{Ca}(\mathrm{OH})_{2}$ slurry is generally greater than that of the dissolved silica solution prepared from sodium silicate, it is necessary to add acid to the slurry to reduce the $\mathrm{pH}$ to the required level before adding this slurry to the dissolved silica solution. The acid that can be used may depend on the ultimate purpose to which the silicate material is to be put. Normally hydrochloric acid is used but in some applications such as anti-corrosion, the chloride ions can be problematic. Generally nitric and acetic acids can be readily used in this and most other applications. Sulfuric acid does have limitations as this can cause the simultaneous and undesirable precipitation of calcium sulfate.

It is also preferable that this solution or slurry is prepared and maintained at room temperature (approximately $15-25^{\circ} \mathrm{C}$ ) to minimize energy costs. However it is recognized that temperatures up to $100^{\circ} \mathrm{C}$ or higher if the system is pressurized, can be used.

Figure 3 shows the effect of changing the concentration of $\mathrm{Ca}^{2+}$ and $\mathrm{OH}^{-}$ions on the development of the nano-structure as measured by the oil absorption. The concentration of $\mathrm{Ca}^{2+}$ and $\mathrm{OH}^{-}$ions are expressed as mole fractions relative to the mole fraction of $\mathrm{Si}$ (normalized to 1) in the nano-structured calcium silicate material. For nano-structured calcium silicates which have been washed with 
2-ethoxyethanol to maintain the integrity of the nano-structure upon drying, the nano-structure and hence the oil absorption capacity is fully developed when the mole fractions of the $\mathrm{Ca}^{2+}$ and $\mathrm{OH}^{-}$ions are close to or preferably equal to that of $\mathrm{Si}$ (normalized to 1) ie $\left[\mathrm{Ca}^{2+}\right]=\left[\mathrm{OH}^{-}\right]=1$.

\section{(c) desirably rapidly and with mixing}

The $\mathrm{Ca}^{2+}$ solution or slurry is desirably rapidly added to the solution of dissolved silica with effective mixing (stirring) wherein the nano-structured calcium silicate in its initial form is precipitated rapidly. When the $\mathrm{Ca}^{2+}$, in the form of a slurry of $\mathrm{Ca}(\mathrm{OH})_{2}$ at the required $\mathrm{pH}$, is used, the undissolved $\mathrm{Ca}(\mathrm{OH})_{2}$ rapidly dissolves to replace the already dissolved $\mathrm{Ca}^{2+}$ ions that have reacted with the dissolved silica species, mainly $\mathrm{H}_{3} \mathrm{SiO}_{4}{ }^{-}$, in the silica solution. Effective stirring should be maintained during the addition process and for a period of up to about 5 minutes thereafter to ensure uniformity and completeness of the precipitation process. During the mixing and precipitation process, the $\mathrm{pH}$ of the nano-structured calcium silicate slurry increases to a $\mathrm{pH}$ of about $12.0-12.5$, typically 12.3 due to the production of $\mathrm{OH}^{-}$ions in the precipitation reaction. With subsequent ageing wherein the $\mathrm{OH}^{-}$ions are incorporated in the nano-structured calcium silicate material, the $\mathrm{pH}$ reduces to about $\mathrm{pH}=10.5-11.5$, typically 11.5 .

Alternatively the silica solution can be added to the calcium hydroxide slurry. The same methodology and limitations apply as for the previously described method. However, as some sources of calcium hydroxide can contain insoluble impurities for example calcium oxide and calcium carbonate, the careful addition of the calcium hydroxide slurry by decantation to the silica solution allows these impurities to be left behind in the vessel containing the slurry. Therefore the addition of a calcium hydroxide slurry to the silica solution as detailed above is the preferred method, but the invention is not limited to this order. 
This process is preferably carried out at room temperature to minimize energy costs and effect ease of addition and mixing.

\section{Ageing of the slurry.}

Following the rapid addition of the $\mathrm{Ca}^{2+}$ containing solution or $\mathrm{Ca}(\mathrm{OH})_{2}$ slurry at the required $\mathrm{pH}$ with effective mixing, the initially formed nano-structured calcium silicate precipitate is rapidly stirred for a further period of about 5 minutes to ensure compete mixing and reaction of the components. Following this the initially formed nano-structured calcium silicate is aged by gently stirring the slurry to ensure continued mixing during which time the framework nano-structure of the calcium silicate material is progressively developed. As a consequence the oil absorption and surface area progressively increase with time. This provides a method for preparing nano-structured calcium silicate materials with particular oil absorption capacities and surface areas.

Figure 4 shows a sequence of electronmicroscope photos detailing the development of the nano-structure of the calcium silicate at ageing times of 10 minutes, 60 minutes and 360 minutes from the addition of the source of $\mathrm{Ca}^{2+}$ at the required $\mathrm{pH}$ to the dissolved silica solution. After 10 minutes only a poorly developed nano-structure is observable which progressively develops over the ageing period. After 360 minutes ageing the nano-structure is approaching that shown in Figure 1 for a fully aged nano-structured calcium silicate material.

The progressive development of oil absorption and surface area with ageing time is shown in Figure 5.

It has also been found that the actual ageing time required to develop the nano-structure varies with the effectiveness of the stirring or mixing during the ageing process. This is governed to some extent by the vessel and stirrer design and in turn by the thickness of the slurry which is related to the initial concentration of the dissolved silica in the starting silica containing solution. For materials prepared from solutions, assuming a $5 \mathrm{~L}$ batch, containing about $35,000 \mathrm{mg} \cdot \mathrm{kg}^{-1} \mathrm{SiO}_{2}$ the ageing period is generally up to about 6 hours. For more dilute solutions the ageing period is shorter and can be as short 
as 1 hour. If the slurry is not stirred, the ageing process still takes place but over a longer timeframe. Figure 6 shows the effect of stirring and vessel size (laboratory scale) on the development of the oil absorption capacity with ageing time. For a small vessel of 0.5 litres capacity with effective stirring, the nano-structure and hence the oil absorption capacity is fully developed after an ageing time of about 2 hours. If the same vessel is not stirred, the nano-structure and oil absorption capacity take about 5-6 hours to develop. If the vessel capacity is increased some 10 times, even with effective stirring the nano-structure and oil absorption capacity take about 6 hours to develop.

\section{Separating the aged precipitate:}

Filtering the aged slurry and washing the filter cake with water to remove any residual solution or dissolved ions, for example $\mathrm{Cl}^{-}$from the added hydrochloric acid and $\mathrm{Na}^{+}$ from the sodium silicate solution, from the pores of the material and provide a filter cake of water washed nano-structured calcium silicate then occurs. This cake can then be dried to remove the water and produce a nano-structured calcium silicate material in powder form that can be optionally further ground to a finer particle size if required.

\section{Optional use of "spacer" compounds:}

As detailed above, the hydrogen bonding between water contained in the pores, the silanol ( $\mathrm{Si}-\mathrm{OH})$ groups and hydrated $\mathrm{Ca}^{2+}$ ions on the surfaces of the nano-size plates is strong enough to partially draw the plates together when the water is removed on drying, thereby partially collapsing the nano-structure and reducing the accessible pore volume and resulting oil absorption, and the accessible surface area. The integrity of the nano-structure can be maintained by displacing the water in the pores by a liquid or solution entity (a spacer compound) which hydrogen bonds to these centres and most importantly has a higher boiling point than water to ensure maximum oil absorption capacity in the dry material is achieved. In this process the water is preferentially removed by evaporation (drying) but at the same time the spacer compound prevents the partial collapse of the open framework structure, thereby maintaining the high pore volume, high oil absorption and high surface area characteristics. Typical examples are 2-ethoxyethanol and 2-methoxyethanol. This is readily achieved by plug flow washing 
the water washed filter cake with for example 2-ethoxyethanol. In the plug flow washing process, after the filter cake formed from the slurry the remaining filtrate solution is removed by filtration until a thin surface layer remains. A volume of water is then added to wash the residual filtrate from the pores of the nano-structured calcium silicate filter cake until again a thin surface layer of water remains. A volume of the spacer compound, for example 2-ethoxyethanol is then washed through the filter cake displacing much of the residual water in the pores of the silicate. Filtration is continued until as much as possible of the 2-ethoxyethanol is removed. The resulting cake is then dried whereupon any residual water and the majority of the spacer compound are removed, with the hydrogen bonded residual spacer compound holding the plates apart thereby maintaining the nano-structure framework. Figure 7 shows the effect of the amount of 2-ethoxyethanol in the plug flow wash water on the development of the oil absorption capacity and surface area respectively of nano-structured calcium silicate. These data show that a single plug flow wash of $100 \%$ ethoxyethanol is needed to displace all the pore water and enable the full development of the nano-structure and the corresponding oil absorption capacity and surface area on drying. 


\section{The optional removal of calcium ions by acid washing.}

While calcium is essential for the formation of the nano-structure, it also compromises the integrity of the structure during drying. As described above, unless a spacer-compound is used, the structure partially collapses upon drying leading to a material with reduced pore volume and surface area. The integrity of the nano-structure can however also be maintained to a significant extent by the removal of $\mathrm{Ca}^{2+}$ ions in the structure that are principally associated with the surface of the nano-size plates by washing the aged nano-structured calcium silicate slurry with acid. The $\mathrm{Ca}^{2+}$ ions are presumably chemically bonded directly to the plate surface on one side to water of hydration molecules on the other side which in turn are hydrogen bonded to the water contained in the pores. As the pore water is removed by evaporation during drying, the strength of the hydrogen bonds draw the plates together thereby similarly partially collapsing the open framework structure and reducing the oil absorption capacity and accessible surface area. The removal of the $\mathrm{Ca}^{2+}$ ions by acid washing which also reduces the $\mathrm{pH}$ of the slurry, removes the propensity for this partial collapse thereby retaining the integrity of the nano-structure on drying. The choice of acids can be the same as those used for adjusting the $\mathrm{pH}$ of the calcium hydroxide slurry as in step 2 (b) above.

Figure 7 shows that the oil absorption capacity is only slightly changed with a $\mathrm{pH}$ reduction from that of $\mathrm{pH}=12$ for the initially formed slurry to about $\mathrm{pH}=10$. During this process the acid is presumably protonating the silanol groups on the surface of the nano-size plates. Reducing the $\mathrm{pH}$ from $\mathrm{pH}=9$ to $\mathrm{pH}=7$ increases the oil absorption capacity significantly, to its fully achievable value. During this progressive acid treatment the calcium content is correspondingly reduced from a $\mathrm{CaO}: \mathrm{SiO}_{2}$ mole ratio of 0.95:1.00 for the initial nano-structured calcium silicate material to a mole ratio of $0.05: 1.00$ in the material that has been acid treated to yield a slurry $\mathrm{pH}$ of about $\mathrm{pH}=6$ (Table 1). At $\mathrm{pH}$ values less than about $\mathrm{pH}=6$, the nano-structured calcium silicate begins to dissolve and the oil absorption capacity is reduced. Spacer compounds such as 2-ethoxyethanol are not needed here to maintain the high oil absorption capacity and surface area. 
Table 1 shows the effect of acid washing on the oil absorption capacity, surface area and the composition of the resulting nano-structured calcium silicate and the resulting mole ratios of $\mathrm{CaO}: \mathrm{SiO}_{2}$ (normalized to 1): $\mathrm{LOI}$ is the loss on ignition of the sample and essentially represents the hydroxyl and water content.

\begin{tabular}{|c|c|c|c|c|c|c|}
\hline & & Oil Absorption & Surface Area & \multicolumn{3}{|c|}{ Molar Ratios } \\
\cline { 3 - 7 } Sample & $\mathrm{pH}$ & $\left(\mathrm{g} .100 \mathrm{~g}^{-1}\right)$ & $\left(\mathrm{m}^{2} \cdot \mathrm{g}^{-1}\right)$ & $\mathrm{CaO}$ & $\mathrm{SiO}_{2}$ & $\mathrm{LOI}=\mathrm{H}_{2} \mathrm{O}, \mathrm{OH}^{-}$ \\
\hline AJM5-82A & 12 & 113 & 60 & 0.95 & 1.00 & 1.36 \\
\hline AJM5-82C & 10 & 148 & 111 & 0.81 & 1.00 & 1.12 \\
\hline AJM5-82D & 8.5 & 294 & 260 & 0.55 & 1.00 & 0.96 \\
\hline AJM5-82E & 7 & 367 & 267 & 0.11 & 1.00 & 0.38 \\
\hline AJM5-82F & 6 & 372 & 317 & 0.05 & 1.00 & 0.28 \\
\hline
\end{tabular}

Table 1: Oil absorption, surface area and composition of the nano-structured calcium silicate acid washed to particular $\mathrm{pH}$ values.

The nano-structured calcium silicate material with a $\mathrm{pH}$ of about $\mathrm{pH}=7-9$ can readily be used in paper filling or other applications where a higher alkalinity is not desired.

\section{The optional reinforcement of the calcium silicate nano-structure.}

The integrity of the nano-structure can also be maintained to a significant extent and partial collapse prevented on drying from the water washed slurry by reinforcing the nano-size plates and interplate contacts. This is achieved by depositing additional silica, (presumably as calcium silicate) onto the plate surfaces and interplate contact areas. For this, additional $\mathrm{H}_{3} \mathrm{SiO}_{4}{ }^{-}$silicate ions, preferably from a sodium silicate solution, are added with effective and gentle stirring to the aged nano-structured calcium silicate slurry. The $\mathrm{H}_{3} \mathrm{SiO}_{4}{ }^{-}$ions react with the $\mathrm{Ca}^{2+}$ and silanol groups on the surface of the plates thereby depositing further calcium silicate directly onto these plates and their interplate contacts reinforcing the nano-structure to the desirable extent where it does not collapse upon drying the water washed cake.

Figure 8 shows the effect of reinforcing the calcium silicate nano-structure with different amounts (moles) of monomeric $\mathrm{H}_{3} \mathrm{SiO}_{4}{ }^{-}$ions for a nano-structured calcium silicate slurry prepared from a dissolved silica solution containing about $35,000 \mathrm{mg} \cdot \mathrm{kg}^{-1}$ 
$\mathrm{SiO}_{2}$, and the corresponding oil absorptions of the water washed and dried filter cake. With progressive reinforcement the oil absorption capacity increases up to a steady value. In practice effective reinforcement can be achieved by adding $\mathrm{H}_{3} \mathrm{SiO}_{4}{ }^{-}$ions in an amount of about $11 \mathrm{~g}$ of $\mathrm{SiO}_{2}$ per $100 \mathrm{~g}$ of nano-structured calcium silicate, from a dissolved silica solution containing about $35,000 \mathrm{mg} \cdot \mathrm{kg}^{-1} \mathrm{SiO}_{2}$. The $\mathrm{H}_{3} \mathrm{SiO}_{4}{ }^{-}$ions react with the $\mathrm{Ca}^{2+}$ and silanol groups on the surface of the plates thereby depositing further calcium silicate directly onto these plates and their interplate contacts reinforcing the nano-structure to the desirable extent where it does not collapse upon drying the water washed cake. Again, spacer compounds such as 2-ethoxyethanol are not needed here to maintain the high oil absorption capacity and surface area. However, they can be used.

\section{The optional coating of the nano-structured calcium silicate by conducting polymers to form novel nano-structured calcium silicate - conducting polymer composite materials.}

It is possible to coat or encapsulate the surface of the nano-structured calcium silicate by conducting polymers, preferably polyaniline, polypyrrole, polythiophene and their various derivatives. This is achieved by immersing the nano-structured calcium silicate in a solution or suspension of the polymer in water or a suitable organic liquid. Examples of this are polymethoxyaniline sulfonate in water, a dispersion of polypyrrole or polyaniline in water stabilized by a suitable dispersant, or in an organic liquid such as acetone.

The conducting polymer coating can also be achieved by the insitu polymerisation of the monomer onto the nano-structured calcium silicate. For this, the aniline, pyrrole or thiophene monomer, or their derivative forms may be applied to the nano-structured calcium silicate, followed by an oxidant for example ferric chloride, ammonium persulfate, hydrogen peroxide or iodine which causes polymerisation of the conducting polymer onto the surfaces of the nano-structured calcium silicate material. Alternatively, the oxidant may be added first followed by addition of the monomer. Some oxidants require aid of a catalyst as their oxidation potential is not high enough to facilitate oxidation directly. For example the oxidative strength of iodine is not high enough to generate polyaniline composites. But in the presence of calcium in the 
calcium silicate, iodine is activated due to forming charge transfer complexes with the calcium and thereby gains the necessary oxidation potential to facilitate polyaniline polymerisation.

UV-visible spectroscopy and thermogravimetric analysis of conducting polymer calcium silicate composites with various calcium contents (prepared by acid washing the calcium silicate to a particular $\mathrm{pH}$ level), suggest that the polymers bind to the calcium and therefore that the amount of polymer incorporated into the composite is directly dependent on the calcium content of the initial silicate used. An overview of molar calcium ratios and polymer content in weight percent, $\%(w / w)$, for polymethoxyaniline sulfonate, PMAS, a derivative of polyaniline, is presented in Table 2. However other polymers prefer other binding sites than calcium and are not effected by a change of $\mathrm{pH}$ to the same extent as PMAS.

\begin{tabular}{|c|c|c|c|}
\hline \multirow{2}{*}{$\mathrm{pH}$} & \multicolumn{2}{|c|}{ Mole Ratios } & \multirow{2}{*}{$\begin{array}{c}\text { PMAS content, } \\
(\% \mathrm{w} / \mathrm{w})\end{array}$} \\
\cline { 2 - 3 } & $\mathrm{CaO}$ & $\mathrm{SiO}_{2}$ & 7.5 \\
\hline 12 & 0.95 & 1.00 & 2.5 \\
\hline 8.5 & 0.55 & 1.00 & 0.5 \\
\hline 7 & 0.11 & 1.00 & \\
\hline
\end{tabular}

Table 2: Mole ratios of calcium and silicate and PMAS content at various $\mathrm{pH}$ levels.

Interestingly, oil absorption capacity and surface area measurements of the novel nano-structured calcium silicate - conducting polymer composite materials showing these to be similar values to those for the precursor nano-structured calcium silicate. This suggests the formation of a novel composite material in which the available specific surface area (surface area per unit weight) of the conducting polymer can be increased significantly over that of a conducting polymer film on a planar substrate (for example glass) or other materials. 
Polyaniline coated nano-structured calcium silicate materials are substantially resistantant to strong acids. Normally acid below a $\mathrm{pH}$ of about $\mathrm{pH}=6$ would dissolve the nano-structured silicate. The observation that a polyaniline coated calcium silicate does not dissolve or incur any weight loss upon treatment with strong acid confirms the integrity and completeness of the polymer coating. Composite nano-structured calcium silicate - polymethoxyaniline sulfonate materials prepared in this way have oil absorption capacities up to about $550 \mathrm{~g} .0 \mathrm{l} .100 \mathrm{~g}^{-1}$ material, and surface areas of up to about $550 \mathrm{~m}^{2} \cdot \mathrm{g}^{-1}$.

Interestingly also, measurement show that these composite materials exhibit the electronic and chemical properties inherent in the conducting polymer, notably:

- The UV-Visible absorption spectra showing the electronic transitions of the conducting polymer;

- Electronic conductivity which, as with conducting polymers, may be enhanced by doping with appropriate ions such as $\mathrm{Cl}^{-}$. This provides a new solid particulate material with electrical conductivity for use either by itself or as a composite with other materials such as plastics, paint, paper or paper packaging to impart electrical conductivity to them for applications in for example anti-static and electrical and electromagnetic shielding applications; - Oxidation-reduction properties, which may be used to recover dissolved species from solution that have a reduction potential consistent with the oxidation potential of the conducting polymer. An example of this is the recovery of silver $(\mathrm{Ag})$ from a solution of silver ions $\left(\mathrm{Ag}^{+}\right)$directly onto the surface of the composite nano-structured calcium silicate - conducting polymer composite. These metal (silver) coated nano-structured calcium silicate - conducting polymer composites in turn are a novel material that can also be used by themselves or incorporated into other material such as plastics, paint, paper and packaging.

In addition, it is known previously that due to the chemical nature and oxidation-reduction potential of conducting polymers such as polypyrrole, they exhibit anti-microbial properties and anti-corrosive properties. The nano-structured calcium silicate - conducting polymer composites 
developed in this invention, particularly when the conducting polymer is polypyrrole, exhibit similar anti-microbial and anti-corrosive properties. Incorporation of these nano-structured calcium silicate - conducting polymer composites into other materials such as plastics, paint, other surface coatings, paper, packaging, fabrics, textiles, medical (antiseptic) dressings and healthcare products, can import anti-microbial or anti-corrosive properties to such materials.

- Hydrophobic barrier coatings, particularly using polyaniline.

\section{The optional functionalisation of the nano-structured calcium silicate by anions, cations and neutral molecules.}

It is possible to bond, adsorb or absorb various anions, cations and neutral molecules into or onto the surface of the nano-size plates of nano-structured calcium silicate, or in the pores. The large surface area and pore volume of the nano-structured calcium silicate material enables significant quantities of these anions, cations and neutral molecules (species) to be accommodated. Examples of these and their particular functionality are listed below.

The open framework of the nano-structured calcium silicate and its ability to offer various binding sites in form of calcium ions and silanol groups (see earlier), enables these species to bond to some extent to the surfaces of the nano-size plates, principally through electrostatic interactions or hydrogen bonding. As such, these species are tethered into the calcium silicate nano-structure. This, together with the accessibility of the pores and surfaces means that such species can still interact with an external environment and provide specific functionality, whilst being stably accommodated in the host nano-structured calcium silicate material. In cases where the tethering is less strong, the particular species may be slowly released to the environment.

The anions, cations or neutral molecules including salts (species) may be incorporated into the pores or onto the plates of the nano-structured calcium silicate material either during the preparation stage of the nano-structured calcium silicate or after it has been formed. For incorporation during the preparation stage, which is particularly relevant to 
anions and cations, these species are added to or dissolved in the required amount in the initial solution containing the dissolved silica prior to addition of $\mathrm{Ca}^{2+}$ ions at the required $\mathrm{pH}$ and the consequent precipitation of the nano-structured calcium silicate. Examples of such species include $\mathrm{Cu}^{2+}, \mathrm{Ag}^{+}, \mathrm{Zn}^{2+}$ cations, and phosphate vanadate, molybdate, zincate anions, neutral salts and magnetic oxides such as magnetite. During the formation of the nano-structured calcium silicate they are adsorbed or absorbed onto the nano-size plates, and/or accommodated in the pores

The incorporation of the functionalizing species after the nano-structured calcium silicate material is formed can be achieved by exposing the dry silicate material to a vapour of the species, for example iodine and sulfur; physically mixing the liquid or a suitable slurry into the dry silicate material, for example perfumes, essential oils, omacide, phenol, chloral hydrate; or adding the nano-structured calcium silicate to a solution or suspension of the species, for example $\mathrm{Cu}^{2+}, \mathrm{Ag}^{+}, \mathrm{Zn}^{2+}$ cations, phosphate vanadate, molybdate, zincate anions and iodine, chlorhexidine, omacide, chloral hydrate; or physically mixing or grinding, a solid into the silicate powder, for example sulfur and iodine.

Examples of these species and the functionality they impart to the nano-structure calcium silicate material include:

- Anti-microbial, anti-fouling and antiseptic properties wherein the active component may include one or more components selected from $\mathrm{Cu}^{2+}, \mathrm{Ag}^{+}$, $\mathrm{Zn}^{2+}, \mathrm{I}_{2}, \mathrm{~S}$ (including polymeric $\mathrm{S}$ ), omacide, chloral hydrate, chlorhexidine and phenols;

- Anticorrosive properties wherein the active component includes one or more compounds selected from phosphate, vanadate, molybdate, zincate, $\mathrm{Cu}^{2+}$, $\mathrm{Ca}^{2+}, \mathrm{Sr}^{2+}, \mathrm{Zn}^{2+}$ etc conducting polymers of various forms;

- Strengthening agents in rubber wherein the active component is $\mathrm{S}$ (including polymeric S);

- Photochemical and photoactive centres selected from the group including $\mathrm{TiO}_{2}, \mathrm{Ti}^{4+}$ and $\mathrm{Ti}^{3+}$ and various rare earth elements and their ions; 
- Heat storage phase change materials which include alkanes, alcohols, organic acids, water, hydrated salts and mixtures thereof, salt solutions and mixtures thereof;

- Gaseous absorption or adsorption materials; with application for example in the absorption of ethylene for the control of fruit ripening, carbon dioxide for removal from air or other recovery including the recovery of ${ }^{14} \mathrm{CO}_{2}$, and hydrogen for storage purposes;

- Perfumes, essential oils and aromatic compounds as air fresheners, deodorants and odour control, relating particularly to the absorption or slow release of the odouriferous material.

\section{The optional incorporation of phase change energy storage materials into the nano-structured calcium silicate material.}

Phase change energy storage materials (PCMs) are those that exhibit a relatively high thermodynamic heat of fusion thereby providing the opportunity to absorb and store a significant quantity of heat in the melting process and release this heat in the solidification process. A major practical problem in utilizing PCMs is the fact that one phase is a liquid and has to be contained. The nano-structured silicate material described here which has a very high oil (liquid) absorption capacity is an ideal material for containing the liquid PCM. A range of novel nano-structured silicate - PCM composite materials have been produced where up to $400 \mathrm{wt} \%$ of PCM can be accommodated in the pores of the silicate with the nano-structured silicate - PCM composite remaining as a solid even though the PCM is present as a liquid in the pores at temperatures above the PCM melting point. This novel solid nano-structured silicate - PCM composite can in turn be mixed into paint, paper, packaging, plastic, cement, plaster, concrete, wood, ceramics etc. to provide passive heat storage and release properties to such consumer products. Details of these materials and their heat storage properties are provided in the applications section below.

The invention will now be described by way of example only with reference to the following Examples: 


\section{Example 1}

Preparation of standard concentration nano-structured calcium silicate and water washed and 2-ethoxyethanol washed forms

Weigh $462.5 \mathrm{~g}$ of calcium hydroxide in a 20 litre plastic container and add 4.6 litres of distilled water. Mix well with a dispersator having a large propeller and gradually add $320 \mathrm{ml}$ of $33 \%$ hydrochloric acid. Carefully wash any powder on the sides of the bucket or the dispersator shaft into the slurry with minimal water. Into a 5 litre plastic beaker weigh $1460 \mathrm{~g}$ of sodium silicate solution and make up to 5 litres with distilled water to give a dissolved silica concentration of $35,000 \mathrm{mg} \cdot \mathrm{kg}^{-1} \mathrm{SiO}_{2}$.

Increase the speed of the dispersator as much as practicable without causing splashing, and rapidly add the 5 litres of sodium silicate solution whereupon the nano-structured calcium silicate precipitates immediately. Continue stirring rapidly to ensure effective mixing and for about 5 minutes. Reduce the stirring speed so that the slurry is being gently mixed and stir for at least 4 and preferably 6 hours. The slurry can then be left to stand for about 12 hours. As it is thixotropic it will thicken on standing and will need to be gently stirred before post treatment and filtration. High shear stirring should not be used as this will damage the nano-structure.

Filter and plug wash the slurry with distilled water to provide a water washed material as a moist filter cake which can then be dried at $110^{\circ} \mathrm{C}$ to provide a powder. The oil absorption of this nano-structured calcium silicate is about $120 \mathrm{~g} .011 .100 \mathrm{~g}^{-1}$ material, and the surface area is about $120 \mathrm{~m}^{2} \cdot \mathrm{g}^{-1}$.

To ensure the integrity of the nano-structure is maintained on drying the water washed filter cake is subjected to a plug wash of 2-ethoxyethanol which acts as a spacer compound. This provides a moist filter cake which can then be dried at $110^{\circ} \mathrm{C}$ to provide a powder. The oil absorption of this nano-structured calcium silicate is about 420 g.oil. $100 \mathrm{~g}^{-1}$ material, and the surface area is about $400 \mathrm{~m}^{2} \cdot \mathrm{g}^{-1}$. 


\section{Example 2}

\section{Preparation of a diluted concentration nano-structured calcium silicate and water}

washed and 2-ethoxyethanol washed forms

The procedure is the same as that for the above standard concentration nano-structured calcium silicate except that $115.5 \mathrm{~g}$ calcium hydroxide, $80 \mathrm{ml}$ of $33 \%$ hydrochloric acid and $378 \mathrm{~g}$ sodium silicate are used. As the resulting slurry is more dilute after the initial rapid mixing period of 5 minutes, it is not necessary to gently mix the slurry for a further 6 hours to ensure development of the nano-structure. This development takes place effectively on standing for about 12 hours.

The oil absorption of the water washed nano-structured calcium silicate is similarly about $120 \mathrm{~g} .0 \mathrm{il} .100 \mathrm{~g}^{-1}$, and the surface area is about $120 \mathrm{~m}^{2} \cdot \mathrm{g}^{-1}$. However, the oil absorption of the 2-ethoxyethanol washed nano-structured calcium silicate is about $550 \mathrm{~g} .0 \mathrm{il} .100 \mathrm{~g}^{-1}$, and the surface area is about $550 \mathrm{~m}^{2} \cdot \mathrm{g}^{-1}$.

\section{Example 3}

\section{Preparation of acid washed nano-structured calcium silicate}

A slurry of aged nano-structured calcium silicate is formed using the procedure detailed in either Example 1 or 2 above. The slurry is then gently and effectively stirred (this is easier for the more dilute slurry - example 2) and acid, preferably hydrochloric is added slowly to the slurry while the $\mathrm{pH}$ of the slurry is monitored. When the desired $\mathrm{pH}$ is reached which is typically $\mathrm{pH}=8-9$, the slurry is left to stand for a few hours. The $\mathrm{pH}$ often increases by $1-2 \mathrm{pH}$ units during this time and the addition of further acid is required to reduce the slurry $\mathrm{pH}$ to the desired value. The amount of calcium remaining in the structure at different final $\mathrm{pH}$ values of the acid washed slurry are given in Table 1 above. The slurry is then filtered and washed with water and optionally dried to give a nano-structured calcium silicate materials with an oil absorption of about $350 \mathrm{~g} .0 \mathrm{ll} .100 \mathrm{~g}^{-1}$, and the surface area is about $260 \mathrm{~m}^{2} \cdot \mathrm{g}^{-1}$. 


\section{Example 4}

\section{Preparation of reinforced nano-structured calcium silicate}

A slurry of aged nano-structured calcium silicate is formed using the procedure detailed in either Example 1 or 2 above. The slurry is then gently and effectively stirred (this is easier for the more dilute slurry - example 2) and sodium silicate is slowly added in an amount about $11 \mathrm{~g}$ of $\mathrm{SiO}_{2}$ per $100 \mathrm{~g}$ of nano-structured calcium silicate. The slurry is then filtered and washed with water and optionally dried to give a nanostructured calcium silicate materials with an oil absorption of about $280 \mathrm{~g} .0 \mathrm{il} .100 \mathrm{~g}^{-1}$, and the surface area is about $250 \mathrm{~m}^{2} \cdot \mathrm{g}^{-1}$.

\section{Example 5}

\section{Preparation of heat energy absorption, storage and release materials}

Heat energy absorption, storage and release material can be prepared by incorporating a phase change material (PCM), typically alkanes or hydrated salts into the pores of the nano-structured calcium silicate material.

In particular, various amounts of the Rubitherm RT25 alkane (paraffin) phase change material (PCM) which melts at about $25^{\circ} \mathrm{C}$, was mixed into a nano-structured calcium silicate in the dry powder form in which the nano-structure was maintained by the 2-ethyoxyethanol spacer compound, to levels of $100 \mathrm{wt} \%, 200 \mathrm{wt} \%$, 300wt $\%$ and $400 \mathrm{wt} \%$ RT25 with respect to the silicate. In all cases the composite energy absorption, storage and release material remained as a free flowing powder. The heat energy absorption and release capacities were measured by differential scanning calorimetry (DSC) and the composite nano-structured calcium silicate - 400wt\% RT25 material shown to have a heat energy absorption and release capacity of about $110 \mathrm{~J}^{\mathrm{g}} \mathrm{g}^{-1}$.

This composite nano-structured calcium silicate - 400wt\% RT25 material was then added to cement in various quantities up to $50 \mathrm{wt} \%$; to paint in various quantities up to $40 \mathrm{wt} \%$, plaster of paris (gypsum plaster such as that used in wall board) in various quantities up to $50 \mathrm{wt} \%$, and to paper as a filler in various quantities up to $20 \mathrm{wt} \%$. DSC measurements were conducted for a number of these composite materials which indeed demonstrate that such novel materials do exhibit significant heat energy absorption, 
storage and release capacities. The cement containing $50 \mathrm{wt} \%$ of the nano-structured calcium silicate - 400wt\% Rubitherm RT25 composite showed a heat storage capacity of about $33 \mathrm{~J} . \mathrm{g}^{-1}$; the paint containing $40 \mathrm{wt} \%$ of the nano-structured calcium silicate $400 \mathrm{wt} \%$ of Rubitherm RT25 composite showed a heat storage capacity of about $45 \mathrm{~J} . \mathrm{g}^{-1}$; and plaster of paris (gypsum plaster) containing $50 \mathrm{wt} \%$ of the nano-structured calcium silicate - $400 \mathrm{wt} \%$ of Rubitherm RT25 composite showed a heat storage capacity of about $45 \mathrm{~J}_{\mathrm{g}} \mathrm{g}^{-1}$.

Similar composite materials using nano-structured calcium silicate and Rubitherm RT20 (melting point about $20^{\circ} \mathrm{C}$ ) were prepared, and added to paint, gypsum plaster and cement in both similar and larger quantities. The heat storage capacities were comparable to those of the Rubitherm RT25 composites.

Because the nano-structured calcium silicate contains hydroxyl groups and usually some occluded water, it can be heated by microwave radiation. Hence a composite nano-structured calcium silicate - PCM material can be readily heated to above the PCM melting temperature by placing the composite in a microwave oven. The effectiveness of the heating can be enhanced by accommodating both water and PCM in the pores of the nano-structured calcium silicate material.

\section{Example 6}

Preparation of composites of nano-structured calcium silicate with iodine and sulfur

Composites of nano-structured calcium silicate with iodine have been prepared by lightly mixing up to about $20 \mathrm{wt} \% \mathrm{I}_{2}$ crystals with nano-structured calcium silicate powder, preferably 2-ethoxyethanol washed, and heating the composite up to about $100^{\circ} \mathrm{C}$, preferably $60-80^{\circ} \mathrm{C}$ for up to $12-24$ hours preferably up to $2-5$ hours in a closed environment. The $I_{2}$ vaporises and diffuses into the pores of the nano-structure and is adsorbed or bonded onto the surface of the nano-size platelets. Further detailed spectroscopy studies suggest the iodine is bonded to the surface calcium ions and may be in the form of a charge transfer complex. If the $\mathrm{Ca}^{2+}$ ions are removed by acid washing only low, if any amounts of iodine can be incorporated stably in the 
nano-structure. The nano-structured calcium silicate - iodine composite material is then heated to a temperature of up to about $80-120^{\circ} \mathrm{C}$ in an open environment wherein the excess or unbonded iodine is removed by vaporisation. The complete removal of the excess or unbonded iodine is most readily determined by the achieving of constant weight during the open environment heating. Heating experiments show that the iodine in the nano-structured calcium silicate - iodine composite is stably bound up to a temperature of $200^{\circ} \mathrm{C}$ and with further heating up to $800^{\circ} \mathrm{C}$ the iodine is progressively lost from the structure (Table 3) The content of $I_{2}$ in the calcium-silica is typically 3-15 $\mathrm{wt} \%$. The compositions of typical iodine calcium-silica materials are shown in Table 3.

Composites of nano-structured calcium silicate with sulfur have been prepared by mixing together, preferably by grinding or milling, nano-structured calcium silicate powder, preferably 2-ethoxyethanol washed, and elemental sulfur, with the sulfur being in an amount up to about $5 \mathrm{wt} \% \mathrm{~S}$. The mix is then heated in a closed environment at a temperature up to about $200^{\circ} \mathrm{C}$ whereupon the $\mathrm{S}$ is adsorbed or bonded onto the surface of the nano-size platelets to form a nano-structured calcium silicate - sulfur composite material. Photoelectron spectroscopy measurements suggest the sulfur is bonded to oxygen and exists in a form similar to sulfate which is presumably coordinated to the surface $\mathrm{Ca}^{2+}$ ions. Heating experiments show the sulfur in the nano-structured calcium silicate - sulfur composite is stably bound up to at least $800^{\circ} \mathrm{C}$ (Table 2). The compositions of typical nano-structured calcium silicate - sulfur composite material are shown in Table 3.

Composites of nano-structured calcium silicate - sulfur-iodine have also been prepared by combining the above two procedures. (Table 3)

\begin{tabular}{|l|c|c|c|}
\hline Host Element & $\begin{array}{c}\text { Temperature } \\
\left({ }^{\circ} \mathrm{C}\right)\end{array}$ & $\begin{array}{c}\text { Iodine Content } \\
(\mathrm{wt} \%)\end{array}$ & $\begin{array}{c}\text { Sulfur Content } \\
(\mathrm{wt} \%)\end{array}$ \\
\hline Iodine & 25 & 7.28 & \\
\hline Iodine & 200 & 7.55 & \\
\hline Iodine & 400 & 5.09 & \\
\hline Iodine & 600 & 2.26 & \\
\hline
\end{tabular}




\begin{tabular}{|l|c|c|c|}
\hline Iodine & 800 & 0.17 & \\
\hline Sulfur & 25 & & 2.68 \\
\hline Sulfur & 200 & & 3.11 \\
\hline Sulfur & 400 & & 3.38 \\
\hline Sulfur & 600 & & 3.39 \\
\hline Sulfur & 800 & & 3.52 \\
\hline Iodine + Sulfur & 25 & 5.09 & 1.77 \\
\hline
\end{tabular}

Table 3: Iodine and sulfur contents of composites with nano-structured calcium silicate

\section{Example 7}

Preparation of composites of nano-structured calcium silicate with titanium dioxide

A dilute slurry of nano-structured calcium silicate powder in iso-propanol was prepared by adding $1 \mathrm{~g}$ of nano-structured calcium silicate powder that had first been exposed to $100 \%$ Relative Humidity environment to ensure water molecules were present in the pores and on the surface of the nano-size platelets, to $50 \mathrm{~mL}$ of iso-propanol in a $100 \mathrm{~mL}$ flask equipped with a magnetic stirrer. The slurry was stirred constantly while an amount of titanium isopropoxide to give a mole ratio of $\mathrm{Ca}$ :Ti of 1:1, was added under a blanket of nitrogen to prevent unwanted titanium dioxide formation by reaction with moisture in the air. The mixture was refluxed for about 18 hours, following which $20 \mathrm{~mL}$ of water was added and the slurry stirred for a further 2 hours. During this overall process, the titanium isopropoxide hydrolysed as the anatase polymorph of titanium dioxide hydrate and was incorporated into the pores and surfaces of the nano-structured calcium silicate material. This composite material was then filtered, dried and calcined at $650^{\circ} \mathrm{C}$ for 18 hours whereupon sub-micron size spherical crystals of anatase were formed in and on the nano-structured calcium silicate. The material was characterized by electronmicroscopy and x-ray diffraction, which confirmed the presence of microcrystals of anatase accommodated in the calcium silicate. The photoactivity was tested by the photodegradation of an organic compound (phenolphthalein) in a 
slurry with the nano-structured calcium silicate - titanium dioxide material under UV light. As a comparison, no photodegradation of phenolphthalein was observed using only nano-structured calcium silicate and UV light. This confirmed the photochemical activity of the nano-structured calcium silicate - titanium dioxide material.

\section{Example 8 \\ Preparation of composite calcium silicate - vanadate for anti-corrosion applications.}

A solution containing $5,000 \mathrm{mg} \cdot \mathrm{kg}^{-1} \mathrm{SiO}_{2}$ was prepared and to this sufficient sodium vanadate $\left(\mathrm{Na}_{3} \mathrm{VO}_{4}\right)$ was added to give a concentration of $1,000 \mathrm{mg} \cdot \mathrm{kg}^{-1}$ Vanadate in the silicate solution. A nano-structured calcium silicate - vanadate composite was precipitated by adding $10,000 \mathrm{mg} \cdot \mathrm{kg}^{-1} \mathrm{Ca}^{2+}$. The resulting slurry was filtered and washed with water. The moist filter cake was mixed directly into a latex paint formulation at levels up to $10 \mathrm{wt} \%$ composite in the paint. However, higher levels can be used. This paint was applied to mild steel plates along with the latex paint as a control. In addition, a similar paint was prepared using a commercially available anti-corrosion agent. A cross was scored through the paint to expose the steel surface for each sample. The painted plates were then subjected to a corrosive environment. The paint containing the nano-structured calcium silicate - vanadate material showed significant corrosion resistance compared with the control. It also showed superior performance to the paint containing the commercial anti-corrosion agent. These results are shown in Figure 9. It is noted that areas where the paint has been removed by a solvent after the tests show essentially no corrosion of the underlying steel surface for the nano-structured calcium silicate - vanadate containing paint, whereas the surface of the control shows significant corrosion, and that with the commercial anticorrosion agent show corrosion intermediate between the two. 


\section{Example 9}

\section{Preparation of hydrophobic nano-structured calcium silicate}

A hydrophobic nano-structured calcium silicate suitable for absorbing hydrophobic liquids, or selectively absorbing hydrophobic liquids in the presence of hydrophillic liquids in the form of suspensions or emulsions has been prepared as follows. The nano-structured calcium silicate powder was placed in a porous container and suspended in a vessel capable of holding a pressure of about 20 atmospheres. A volume of 1-butanol was placed in the vessel to a level below that of the porous container. The vessel was sealed and heated to a temperature of $200^{\circ} \mathrm{C}$ for 2 hours, then cooled and opened. During heating, the 1-butanol vapourised and reacted with the silanol groups on the surface of the nano-size platelets rendering the surface hydrophobic. The resulting hydrophobic nano-structured calcium silicate powder was removed from the porous container. When sprinkled on water, the material floated demonstrating its hydrophobic nature.

\section{Examples of Applications}

The following are examples of applications of nano-structured calcium silicate material in its various forms and various functionalised:

\section{Application Example 1}

\section{Use in paper filling to enhance opacity and reduce print through.}

Nano-structured calcium-silicate having an oil absorption of about $350 \mathrm{~g} .0 \mathrm{oil} .100 \mathrm{~g}^{-1}$ has been successfully tested as a filler in $45 \mathrm{gsm}$ and $55 \mathrm{gsm}$ newsprint made from $100 \%$ thermomechanical pulp (TMP), with filler loadings of about $2 \mathrm{wt} \%$ and $4 \mathrm{wt} \%$. Similar tests were carried out using calcined clay, ground calcium carbonate (GCC) $(90 \%<$ 2 microns) and an aluminosilicate Sipernat 820A, for comparison purposes. The optical and physical properties were measured on calendered sheets. The results for $55 \mathrm{gsm}$ newsprint are shown graphically in figure 10 . 
The results (figure 10) show that the increase in opacity with filler loading for $45 \mathrm{gsm}$ and 55gsm TMP newsprint filled with nano-structured calcium silicate and calcined clay are similar, and significantly better than GCC or Sipernat 820 . At a $2 w t \%$ loading, calcined clay and nano-structured calcium silicate increase the opacity of $45 \mathrm{gsm}$ newsprint by about 3.2 points and of $55 \mathrm{gsm}$ newsprint by about 1.3 points. These increases are about twice that provided by GCC and about 3 times that provided by Sipernat 820 .

The high oil absorption capacity of the nano-structured calcium silicate is particularly effective in reducing print through (the printed image showing through to the reverse side of the sheet). For both $45 \mathrm{gsm}$ and $55 \mathrm{gsm}$ TMP newsprint the nano-structured calcium silicate has substantially outperformed calcined clay and GCC, and is also significantly better than Sipernat 820, particularly for 55gsm newsprint. At a $2 \mathrm{wt} \%$ filler loading, nano-structured calcium silicate reduces print through by about $40 \%$ for $55 \mathrm{gsm}$ newsprint and by an impressive $51 \%$ for $45 \mathrm{gsm}$ newsprint, the latter being quite remarkable (figure 10).

The nano-structured calcium silicate material claimed here is therefore an effective filler in increasing the opacity of newsprint sheet and substantially reducing print through.

\section{Application Example 2}

Use in a paper coating formulation to improve print quality, especially for ink-jet printing.

Any of the nano-structured calcium silicate products of the invention in the form of a moist filtercake has been added to a coating formulation and applied to the surface of a paper sheet. The sheet was then printed using a colour ink-jet printer. The colour definition, sharpness and clarity of print were significantly improved over that for the same image printed on uncoated paper. This material is particularly suitable to application in the size press stage of a paper making operation. 


\section{Application Example 3}

\section{Use as an inert carrier and slow release agent for liquids}

(a) Essential oils, perfumes and aromatics

The essential oils, pine oil and clove oil have been mixed into and absorbed in the pores of separate samples of dry nano-structured calcium silicate. These were placed in open dishes. Similar quantities of the pine oil and lavender oil were also placed in open dishes. All dishes were left in the open and the odours emanating from them monitored by smell over a period of about 1 year. During this time the aromas evolved by the pine and lavender oils in the open dishes were initially stronger than the aromas evolved by the oils contained in the nano-structured calcium silicate. However after a period of about 3 months the aromas from the pine and lavender oils in the open dishes were barely detectable as most of the active aroma compounds had largely evaporated in this time. In contrast, the oils contained in the nano-structured calcium silicate continued to evolve aromas that were readily detectable by smell. As such, it is clear that the nano-structured calcium silicate material is an effective slow release agent for essential oils and other aromatic compounds.

(b) Odoriferous repellent compounds

In addition, the liquid active ingredients of the animal repellants, notably crotyl mercaptan and also isoamyl mercaptan and butane thiol have been absorbed into nanostructured calcium silicate thereby making these compounds in a solid rather than liquid or paste form. Such a solid form can be easily spread around lawns, gardens etc where animals are not wanted. Also, the nano-structured calcium silicate affords the slow release of these active compounds.

\section{Application Example 4}

Use as a high absorbent material for absorbing and cleaning up liquid spills for example food, wine, oil etc.

Any of the nano-structured calcium silicate powders of the invention have been shown to be effective in cleaning up liquid spills, for example food colourants, sauces, 
beverages, wine etc; oils and other liquids, from carpet and other flooring materials and fabrics. Ideally the nano-structured calcium silicate powder should be applied in excess to the liquid spill immediately after the spill occurs, whereupon the liquid is quickly absorbed into the large pore volume of the silicate. However, if the liquid spill has soaked into the substrate material, the nano-structured calcium silicate powder can be worked into the pile of the carpet or pores in the fabric etc where it is effective in absorbing the liquid that has soaked in. If excess silicate is used, the resulting silicate-liquid material remains as a powder and can then be removed by vacuum suction. Repeated applications of the nano-structured calcium silicate may be required to remove the liquid or significantly minimize its undesirable impact.

\section{Application Example 5}

Use as an absorbent or adsorbent in recovering metal ions and anions such as phosphate, vanadate, molybdate, zincate, aluminate, technatate, rhenate etc from solutions containing these dissolved species.

Nano-structured calcium silicate has shown to be effective in adsorbing metal ions and anions from solutions, particularly when they are in low concentrations of a few hundred mg. $\mathrm{kg}^{-1}$ or less. The moist filter cake form of the water washed nano-structured calcium silicate may be used directly as the open framework structure and hence accessibility to the large pore volume and surface area is maintained since the material is not dried. The 2-ethyoxyethanol washed material can be used in either the moist filter cake form or the dry form.

For example, $1 \mathrm{~g}$ (dry weight basis) of water washed nano-structured calcium silicate moist filter cake was added to a 1 litre solution containing $100 \mathrm{mg} \cdot \mathrm{kg}^{-1}$ each of dissolved silver, copper and zinc metal ions. The solution with the silicate suspension was stirred and samples of the solution taken at time intervals of 15, 30, 60 and 180 minutes from the silicate addition. These samples were analysed for their silver, copper and zinc contents. The results (table 4) show that after 15 minutes the majority of the silver, copper and zinc have been removed by the nano-structured calcium silicate. After 60 minutes the levels in solution are: silver $-0.05 \mathrm{mg} \cdot \mathrm{kg}^{-1}$, copper -0.11 
mg. $\mathrm{kg}^{-1}$, and zinc -0.06 mg. $\mathrm{kg}^{-1}$, which demonstrate the substantial effectiveness of nano-structured calcium silicate as an adsorbent of these metals from solution. This has important application in cleaning up industrial waste water and mine water streams. The nano-structured calcium silicate material containing the adsorbed metal ions can be removed by filtration and dissolved in a small quantity of acid to yield concentrated solution of the metals for ensuing metal recovery and recycling by conventional methods such as electrolysis.

\begin{tabular}{|c|c|c|c|}
\hline \multirow{2}{*}{ Time (minutes) } & \multicolumn{3}{|c|}{ Residual Concentration in Solution } \\
\cline { 2 - 4 } & Silver $\left(\mathrm{mg} \cdot \mathrm{kg}^{-1}\right)$ & Copper $\left(\mathrm{mg} \cdot \mathrm{kg}^{-1}\right)$ & Zinc $\left(\mathrm{mg} \cdot \mathrm{kg}^{-1}\right)$ \\
\hline 0 & 100 & 100 & 100 \\
\hline 15 & 1.7 & 0.12 & 0.14 \\
\hline 30 & 0.05 & 0.11 & 0.06 \\
\hline 60 & 0.05 & 0.06 & 0.04 \\
\hline 180 & 0.04 & 0.05 & 0.03 \\
\hline
\end{tabular}

Table 4: The residual concentrations of silver, copper and zinc ions in solution following the addition of nano-structured calcium silicate to the solution to adsorb these ions.

\section{Application Example 6}

\section{Use as an absorbent of water vapour and in passive humidity control.}

Nano-structured calcium silicate powder, particularly the 2-ethoxyethanol form can be used to absorb and release water vapour and provide a measure of passive humidity control to the immediate environment.

This has been demonstrated by placing a sample of dry nano-structured calcium silicate treated with 2-ethoxyethanol in a closed environment where the relative humidity was maintained at $92 \% \mathrm{RH}$ by a saturated salt solution of potassium nitrate at room temperature. The increase in weight due to the uptake of water was monitored over an 87 day period. During this time the nano-structured calcium silicate essentially absorbed its own weight of water vapour (194\% increase), (figure 11). The increase in 
weight is approximately exponential with the passage of water into the pores being diffusion controlled. There is approximately a $25 \%$ weight gain in the first 8 hours and approximately a $50 \%$ weight gain in the first 48 hours. The water laden sample was then removed from the $92 \% \mathrm{RH}$ environment and placed in a similar closed environment where the relative humidity was maintained at $51 \% \mathrm{RH}$ by a saturated salt solution of calcium nitrate. Again the weight change was monitored with time. The results (figure 11) show that approximately $25 \%$ of the water in the nano-structured calcium silicate was lost in the first 4 hours and approximately $50 \%$ was lost in the first 20 hours. These data show that the nano-structured calcium silicate responds to the relative humidity of the environment by absorbing and hence removing water vapour from a high humidity environment, and also releasing it back to a low humidity environment with a response time of several hours. Hence it is useful as a medium to provide a measure of passive humidity control.

\section{Application Example 7}

\section{Use in heat storage and release applications.}

Examples of the preparation and use of composites of nano-structured calcium silicate with phase change materials for heat storage and release applications are given in Example 5 above.

\section{Application Example 8}

\section{Use in the control of fruit ripening and prolonging the shelf life of the fruit.}

Nano-structured calcium silicate can be used to absorb ethylene gas emitted from fruit during the ripening process and also the carbon dioxide emitted as a consequence of the ripening. The effectiveness of nano-structured calcium silicate in prolonging the shelf life of fruit has been successfully demonstrated. For this, samples of nectarines, apricots, bananas, peaches and pears were each sealed in a plastic bag with a $1 \mathrm{~g}$ quantity of nano-structured calcium silicate contained in a porous sachet. Similar samples of these fruits were each sealed in plastic bags without the nano-structured calcium silicate to serve as respective controls. The plastic bags were placed in a 
sunlight environment and the extent of degradation of the fruit was monitored visually with time. In all cases areas of decay were observed in the control samples about 1-2 weeks before they were observed on the samples in the bags with the sachets of nanostructured calcium silicate. In all of the control samples decay was observable after about 1 to 2 days. Also, the spread of decay was much more rapid in the control samples. These results are shown visually in figure 12 .

Following these demonstrations, the samples of nano-structured calcium silicate were removed from the sachets and analysed. The gas evolved on heating was shown by mass spectrometry to contain ethylene confirming the ability of the nano-structured calcium silicate to absorb this gas which is emitted by ripening fruit. In addition, IR analysis of this silicate material showed the presence of carbonate peaks which presumably result from the absorption of carbon dioxide evolved by the ripening fruit reacting with the hydrated $\mathrm{Ca}^{2+}$ on the surface of the platelets to form calcium carbonate in the pores.

Nano-structured calcium silicate is therefore effective in controlling fruit ripening and extending the shelf life of the fruit.

\section{Application Example 9}

Use as a material with a high surface area in photocatalysis and photoactive applications.)

Examples of the preparations and use of a composite of nano-structured calcium silicate with titanium dioxide as a high surface area photochemical agent are given in Example 7 above. 


\section{Application Example 10}

Use as a hydrophobic material for selectively absorbing oil floating or suspended in water, or from an oil-water emulsion.

Hydrophobic nano-structured calcium silicate prepared according to preparation method in example 9 above, was mixed into an emulsion of oil in water. The hydrophobic nano-structured calcium silicate selectively absorbed the oil and settled to the bottom of the container. Due to the large pore volume, the material can accommodate a similarly relatively large volume of oil. The resulting water, now essentially free of oil was decanted off. This demonstrates the effectiveness of hydrophobic nano-structured calcium silicate in selectively absorbing oil in the presence of water.

\section{Application Example 11}

\section{Use as an anti-microbial agent}

Nano-structured calcium silicate composites with iodine and sulfur prepared according to preparation method in example 6 above, can be used as anti-microbial agents. Their anti-microbial activity has been demonstrated by sprinkling these materials onto half the surface of slices of bread and placing the bread in an environment conducive to the growth of mould for a period of 10 days. A slice of bread with no silicate was used as a control. The results are shown in figure 13. The anti-microbial action of the nano-structured calcium silicate composites with iodine and sulfur are clearly evident. No mould has grown where these materials have been sprinkled on the bread surface. When compared with the control sample it is also evident that the anti-microbial effect, particularly for the nano-structured calcium silicate composite with sulfur extended beyond the area where the material was sprinkled on the bread. This demonstrates the effectiveness of the nano-structured calcium silicate composites with iodine and sulfur as anti-microbial agents. 
It is likely that these materials can be incorporated into medical dressings to provide anti-microbial activity, in paints to provide a sterile painted surface and in sterile packaging.

\section{Application Example 12}

\section{Use in nutraceutical applications}

The high pore volume and oil absorption capacity of the nano-structured calcium silicate material has applications in nutraceutical products where inert carrier and/or liquid absorption properties are required. In particular it can be used as an absorbent in deodorants and skin care products that absorb unwanted or odorous body oils and sweat. In addition, the near neutral $\mathrm{pH}$ of body skin will engender the release of calcium that can then be absorbed through the skin. The material can also be used as a bath salt to similarly absorb body liquids and provide a source of calcium.

The product can also be used as a carrier of body lotions and skin care preparations.

While this invention has been described with reference to preferred embodiments it is not to be construed as limited thereto. Furthermore where specific materials or steps in a process have been described and known equivalents exist thereto, such equivalents are incorporated herein as if specifically set forth. 

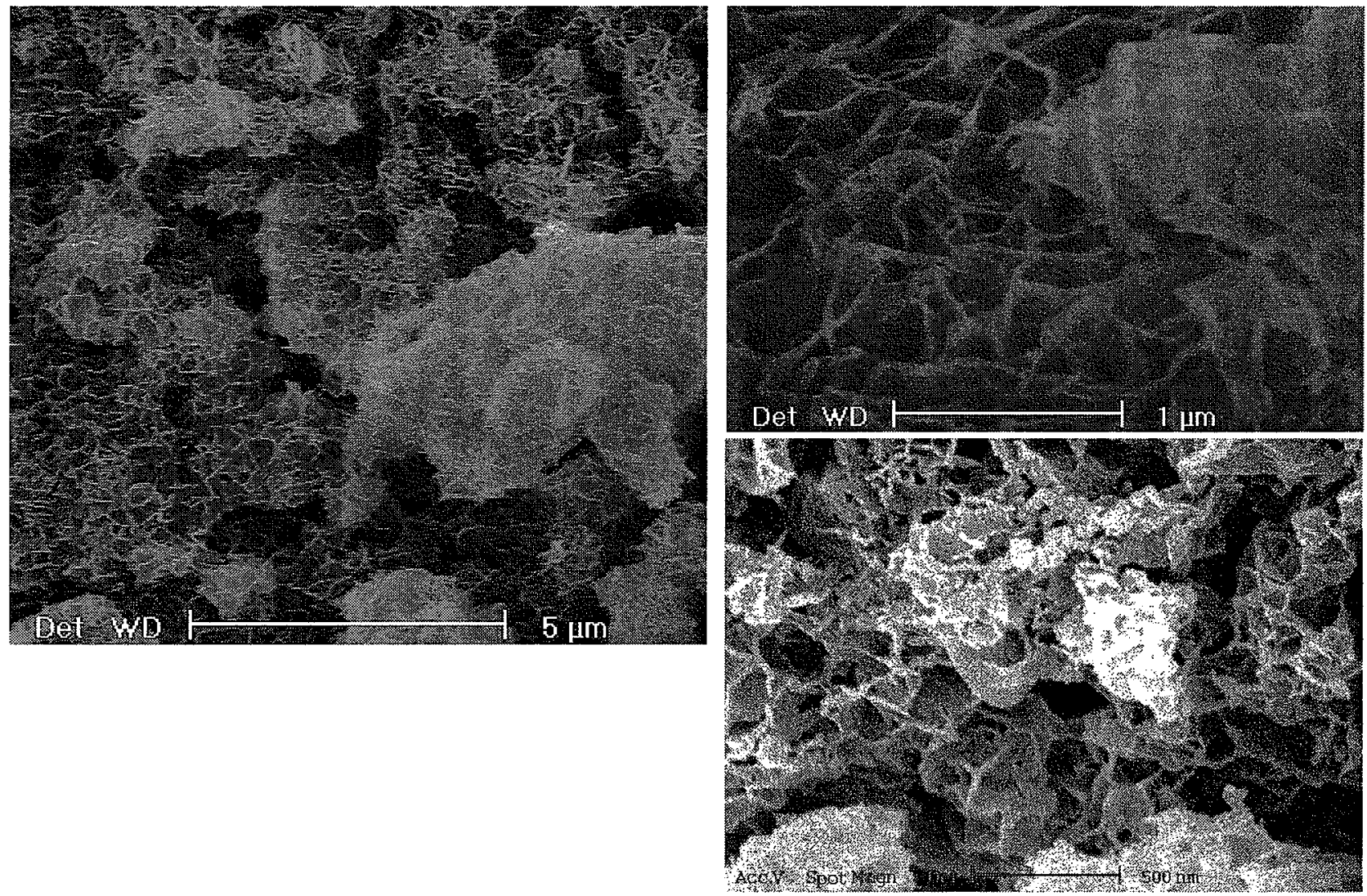

Figure 1: Electronmicroscope photos of nano-structured calcium silicate showing particles comprising nano-size plates stacked in open framework structure which provide a high accessible pore volume and high accessible surface area to the material. 


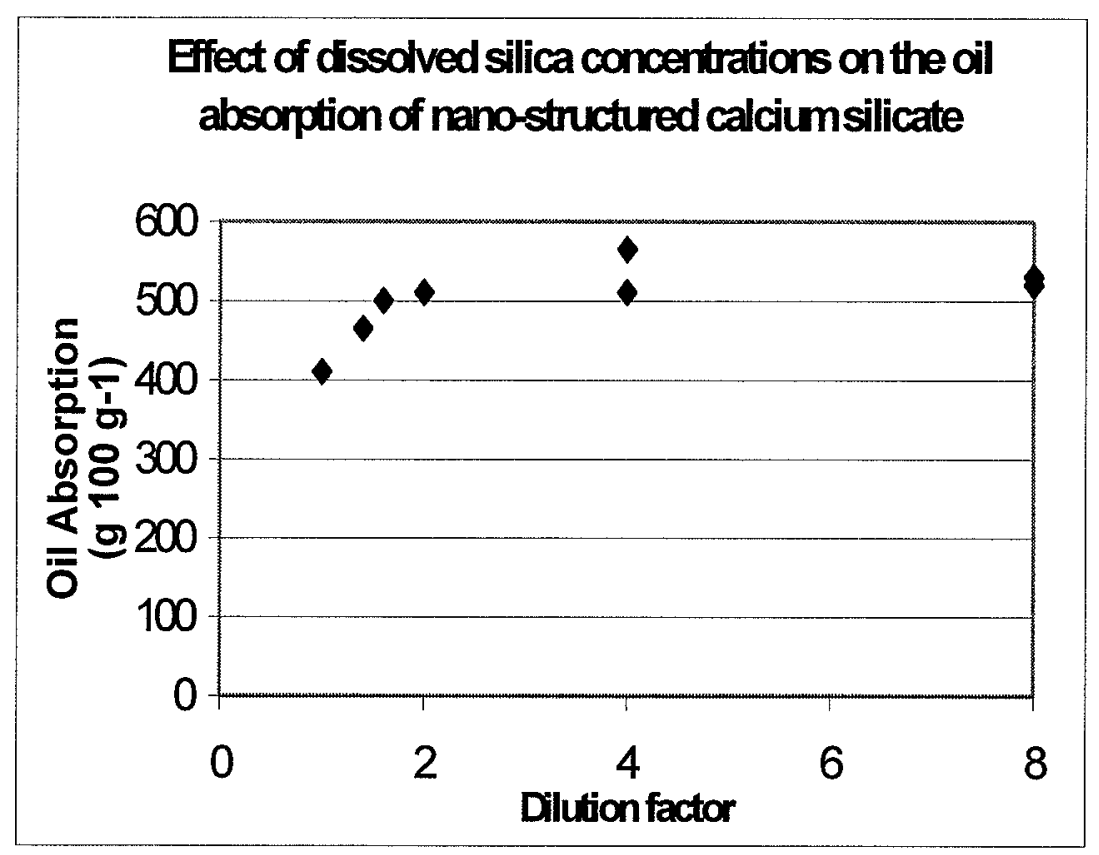

Figure 2: The effect of the concentration of dissolved silica on the development of the oil absorption and hence the nano-structure in nano-structured calcium silicate. The base case (dilution factor $=1$ ) is a dissolved silica concentration of 35,000 mg.kg ${ }^{-1} \mathrm{SiO}_{2}$. 


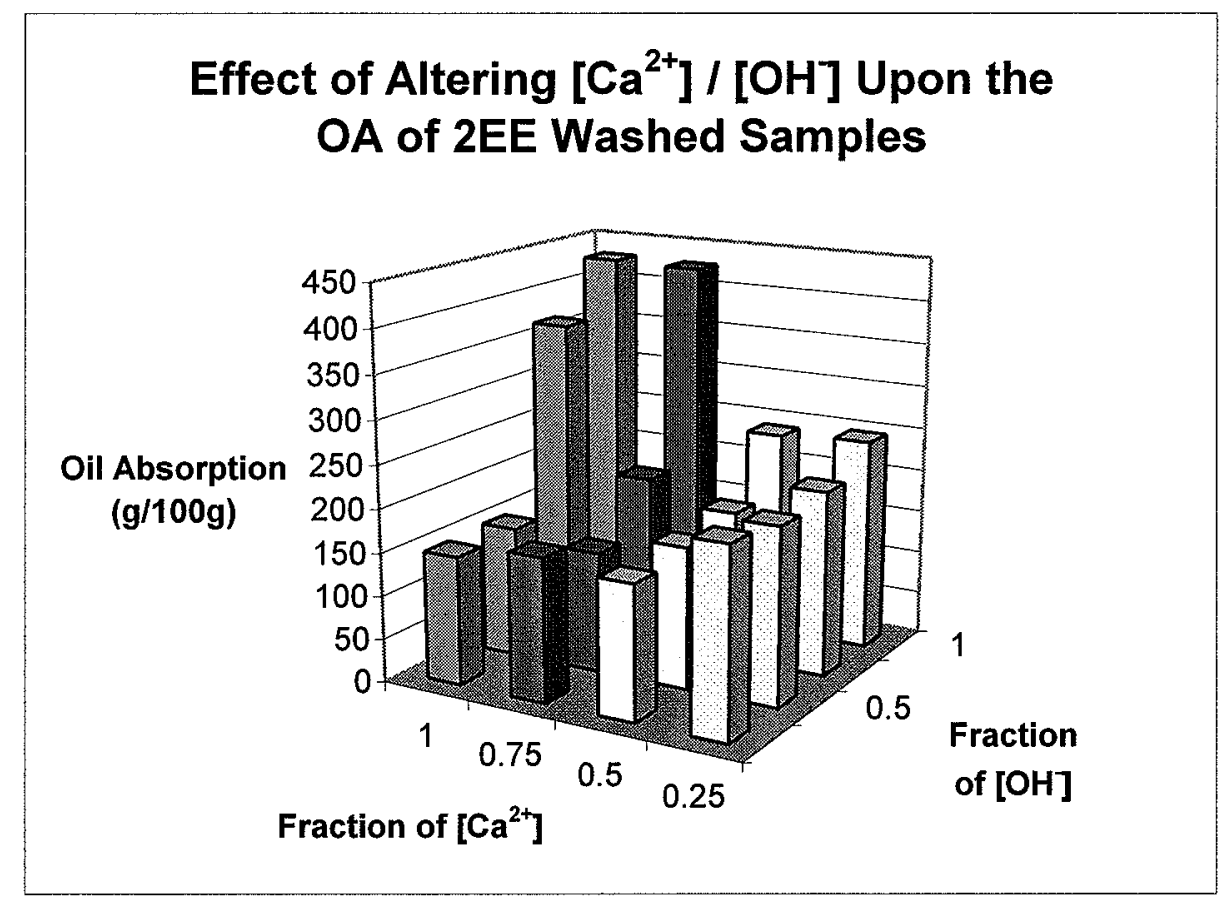

Figure 3: The effect of varying the mole fraction of $\mathrm{Ca}^{2+}$ and $\mathrm{OH}^{-}$in reference to $\mathrm{Si}$ on the oil absorption of nano-structured calcium silicate in which the integrity of the nanostructure has been maintained by treatment with 2-ethoxyethanol. 


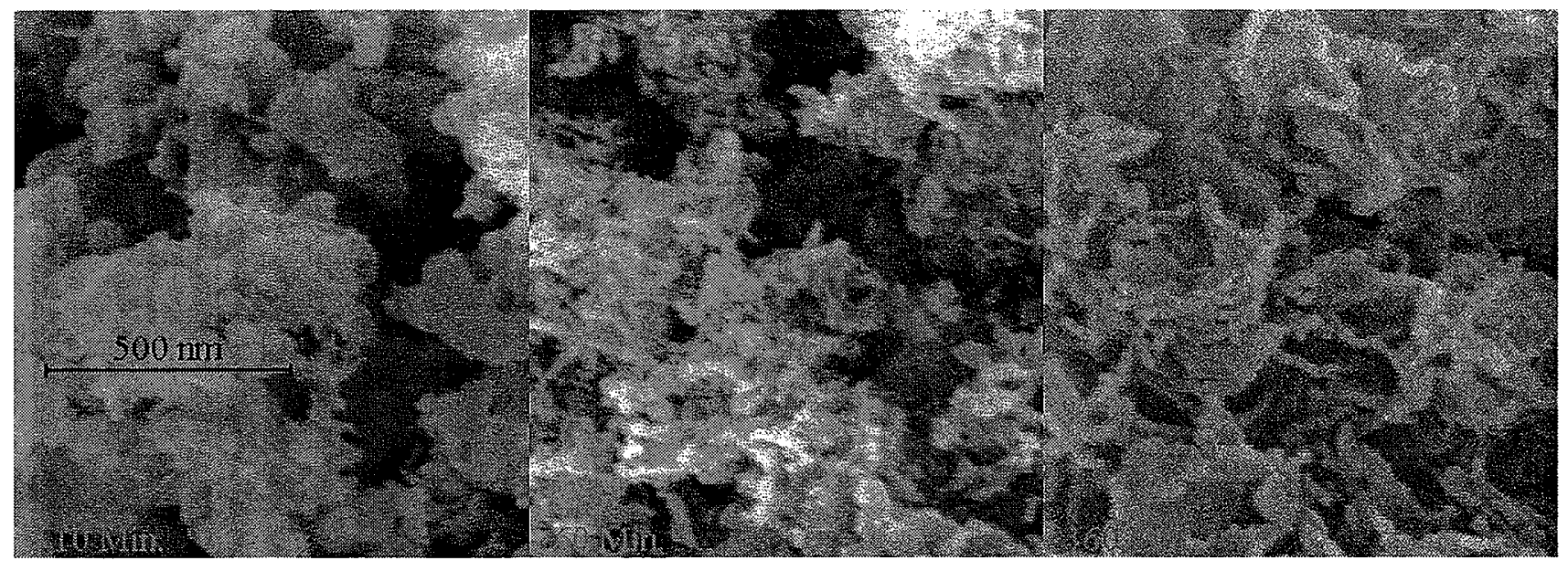

Figure 4: Electronmicroscope photos detailing the development of nano-structured calcium silicate with ageing time. 


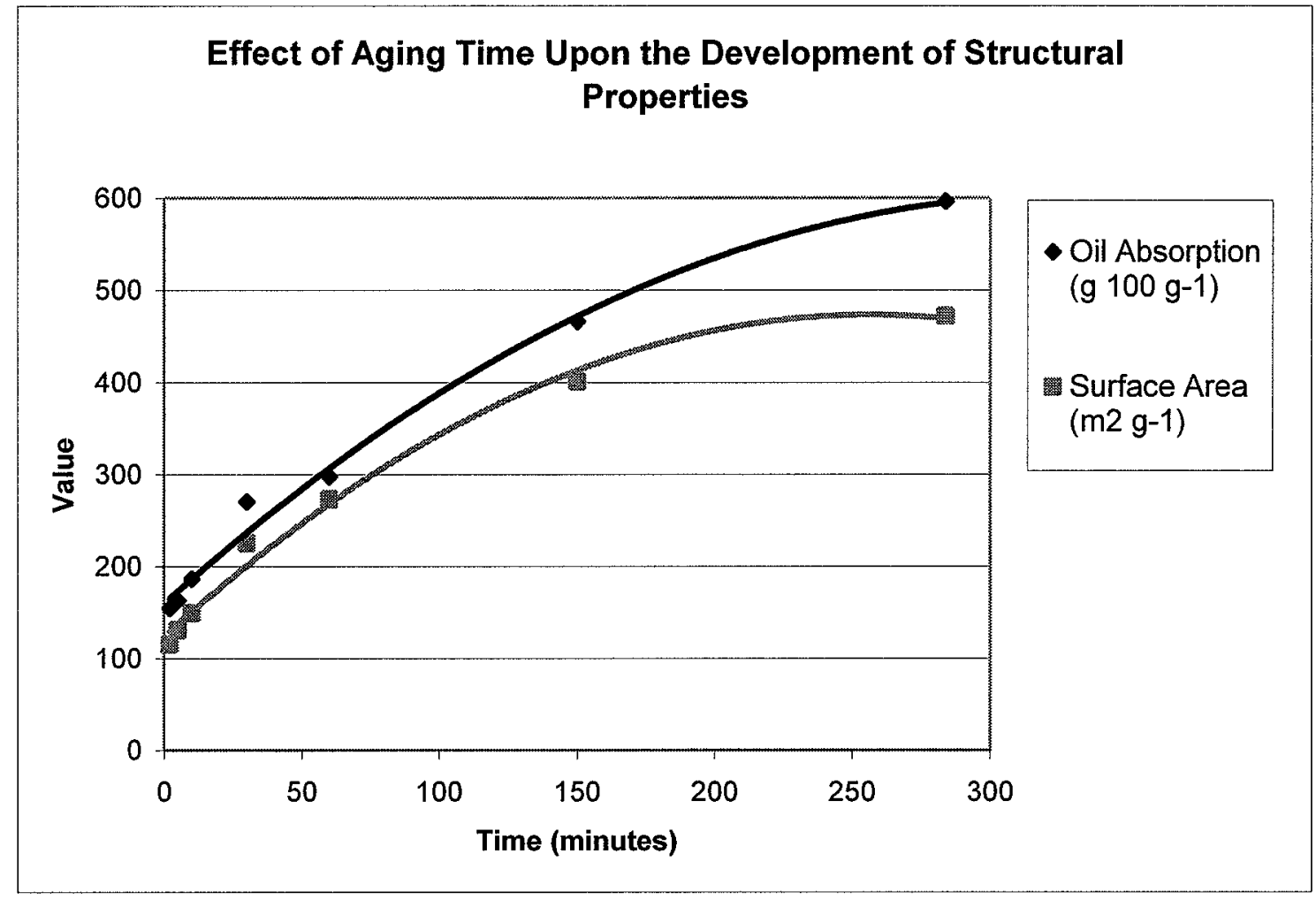

Figure 5: The effect of ageing time on the development of the oil absorption capacity and surface area of nano-structured calcium silicate. 


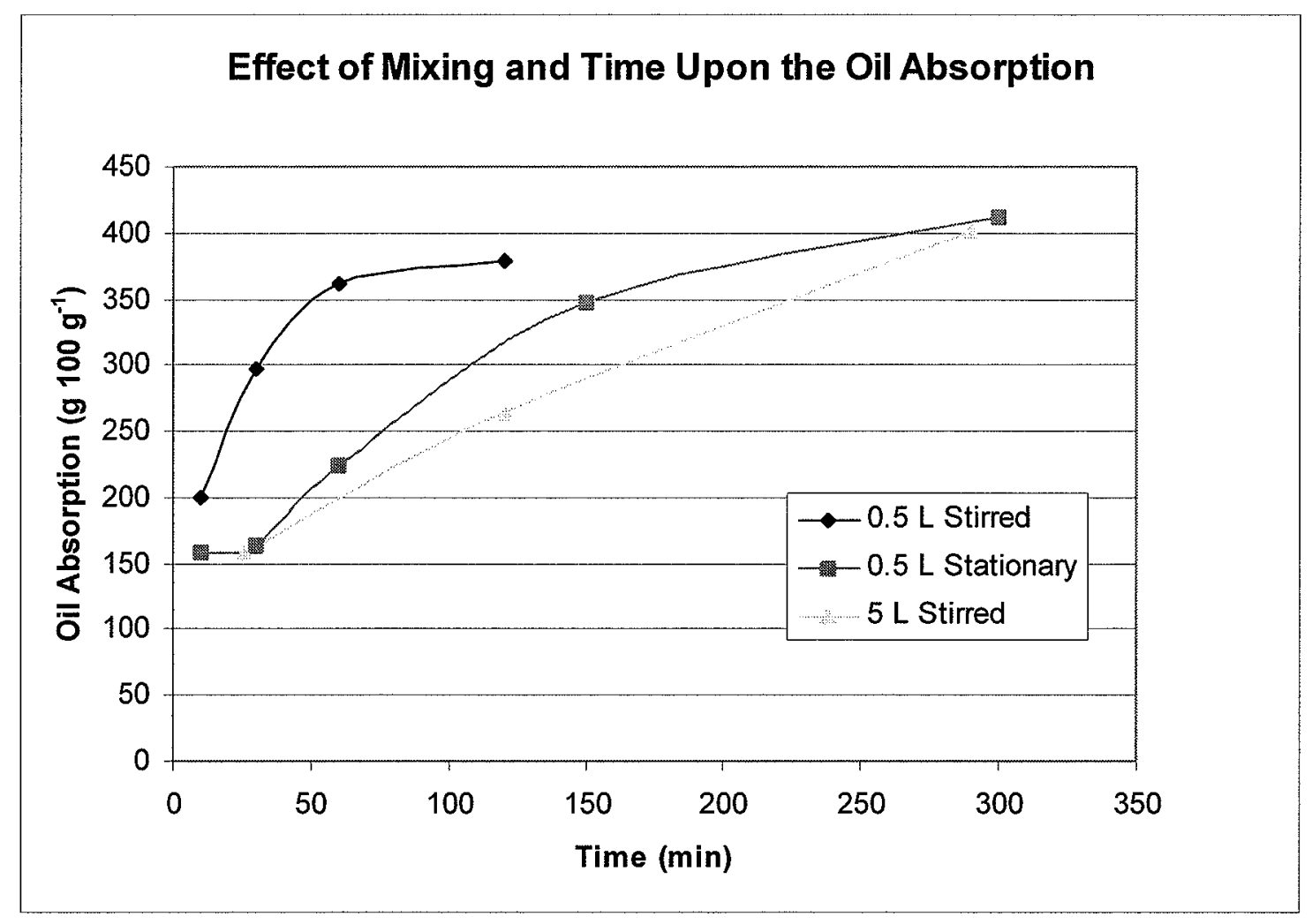

Figure 6: The effect of stirring and vessel size (laboratory scale) the development of the oil absorption capacity and surface area of nano-structured calcium silicate. 

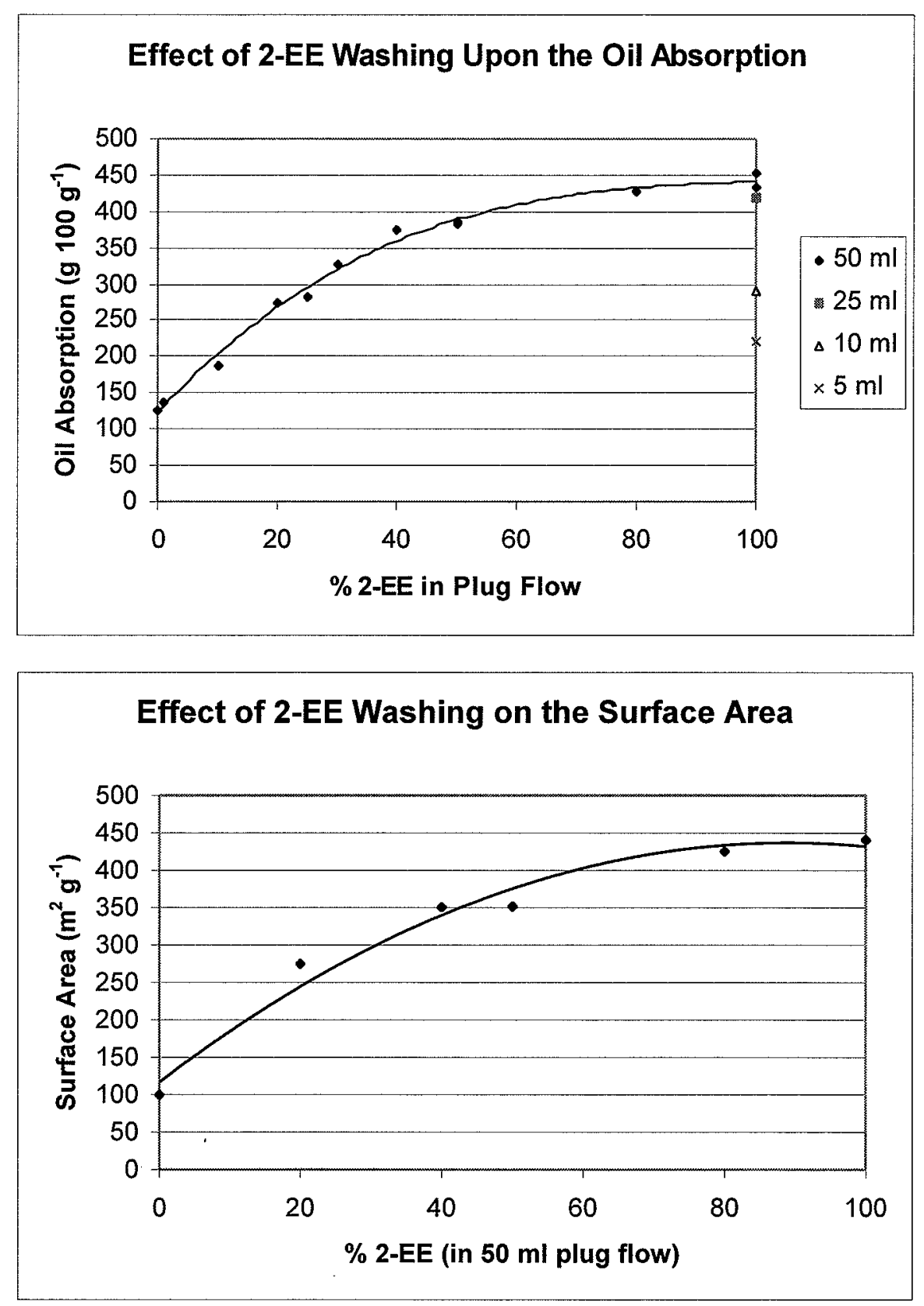

Figure 7: The effect of washing the nano-structured calcium silicate precipitate with 2-ethoxyethanol on the development of the oil absorption capacity and the surface area of the material. 


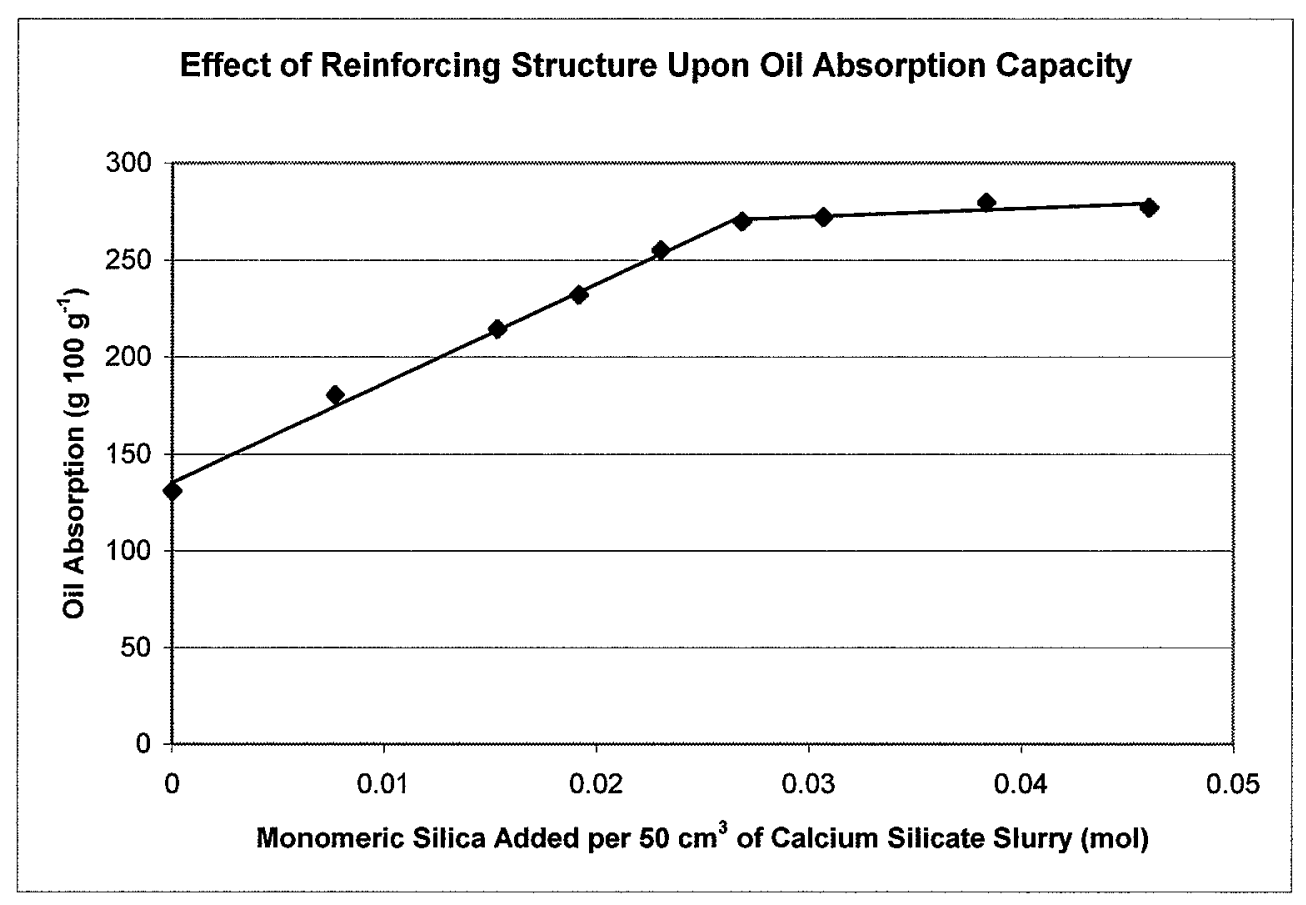

Figure 8: The effect of reinforcing the nano-structure of the calcium silicate material with monomeric silica upon the oil absorption capacity. 


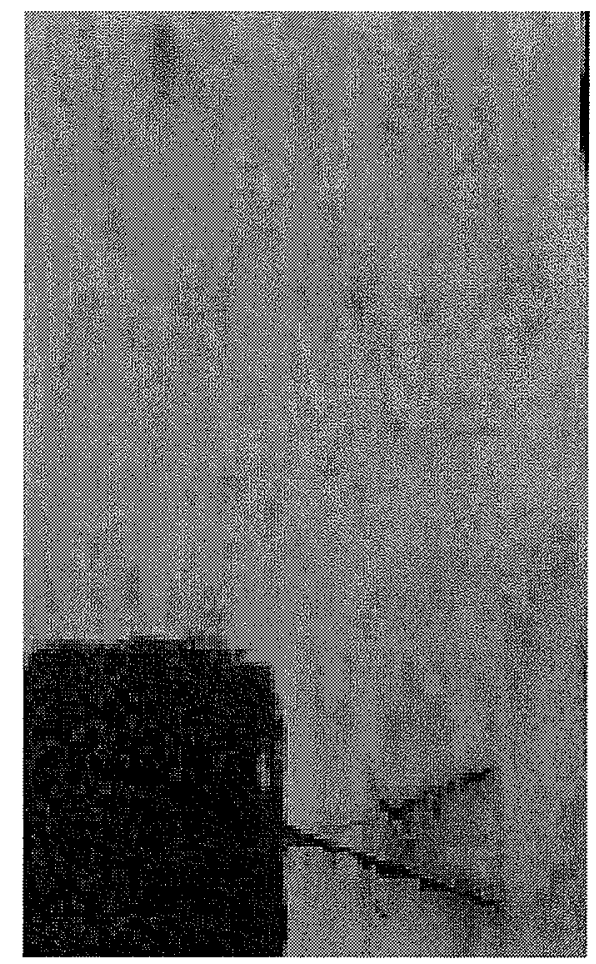

Latex paint - control

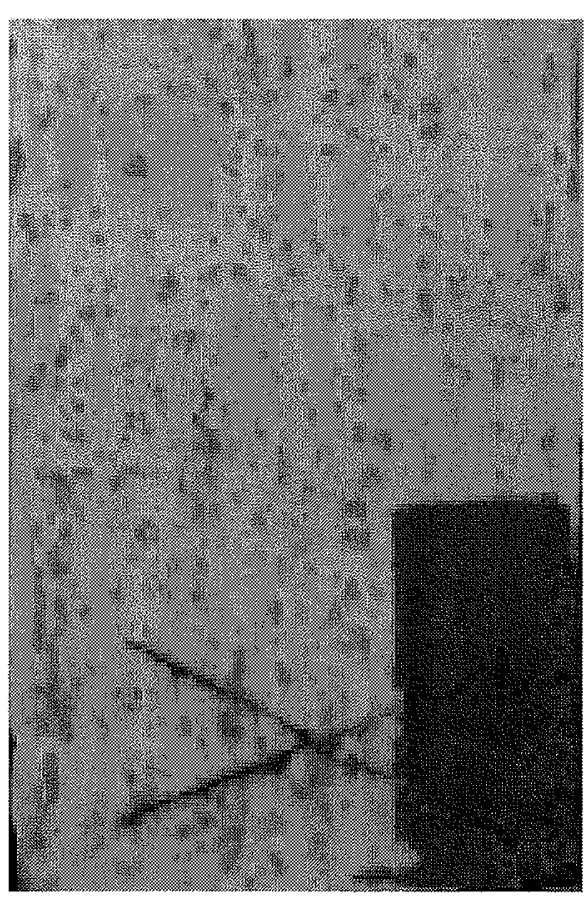

Latex Paint with a commercial

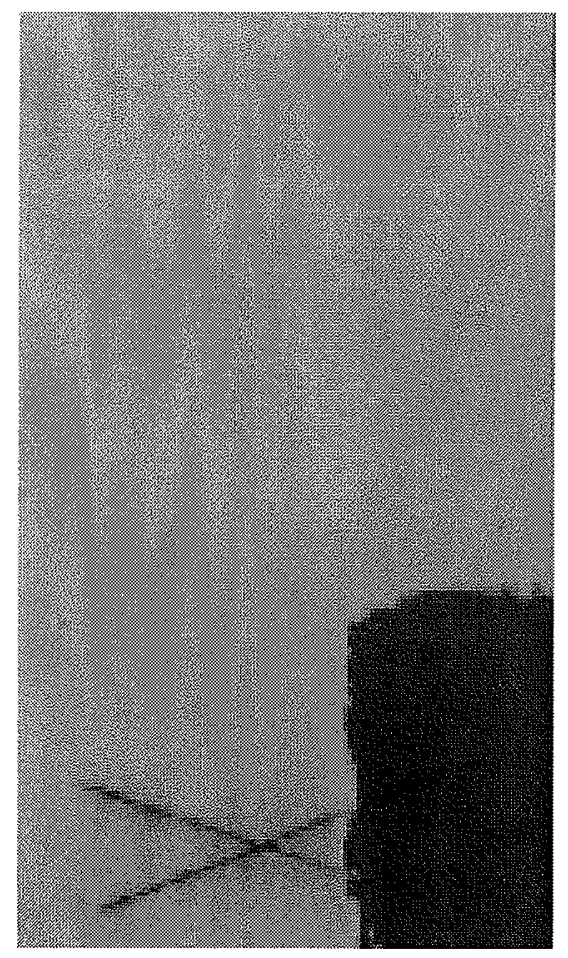

Latex paint with nano-structured

calcium silicate - vanadate

Anti-corrosion agent

Figure 9: The performance of nano-structured calcium silicate vanadate as an additive to latex paint in the prevention of corrosion on mild steel, in comparison with a latex control and a similar latex paint prepared with a commercial anti-corrosion agent. 

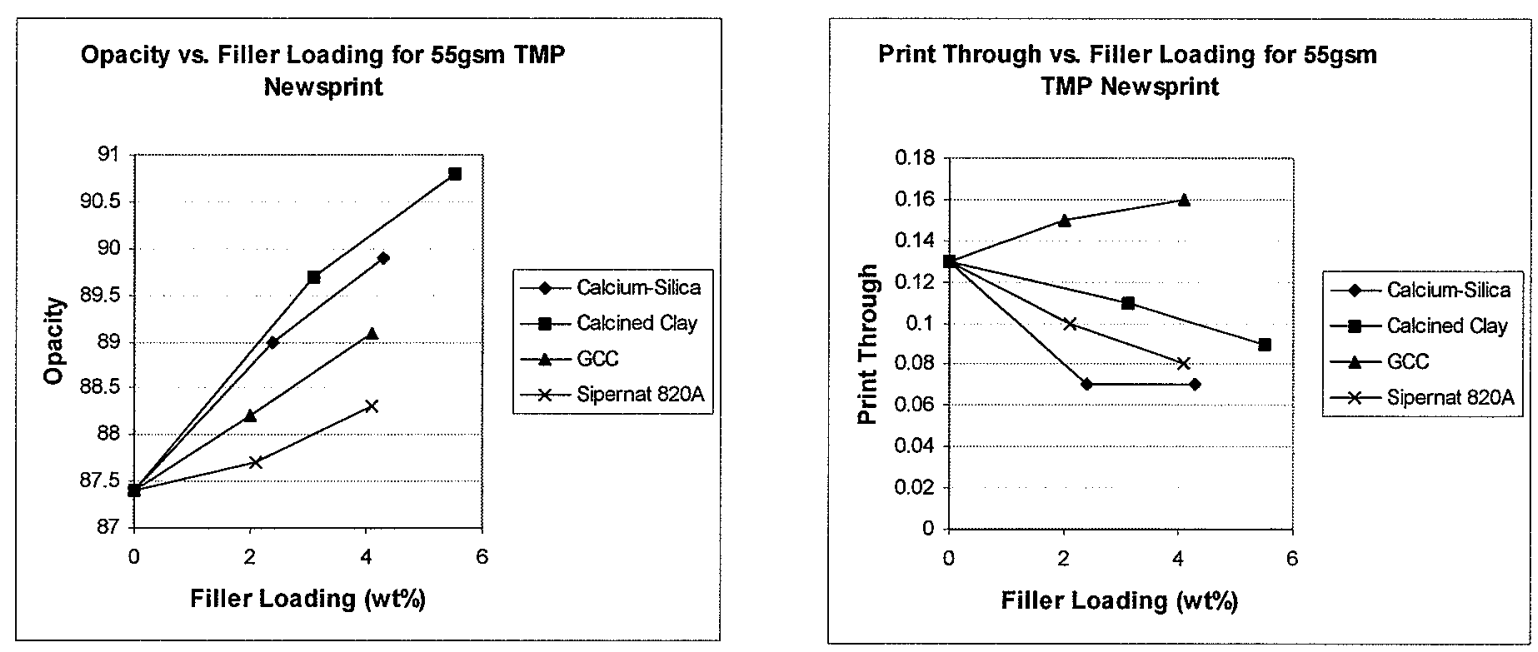

Figure 10: The performance of nano-structured calcium silicate as a filler in $55 \mathrm{gsm}$ newsprint in increasing opacity and reducing print through. 


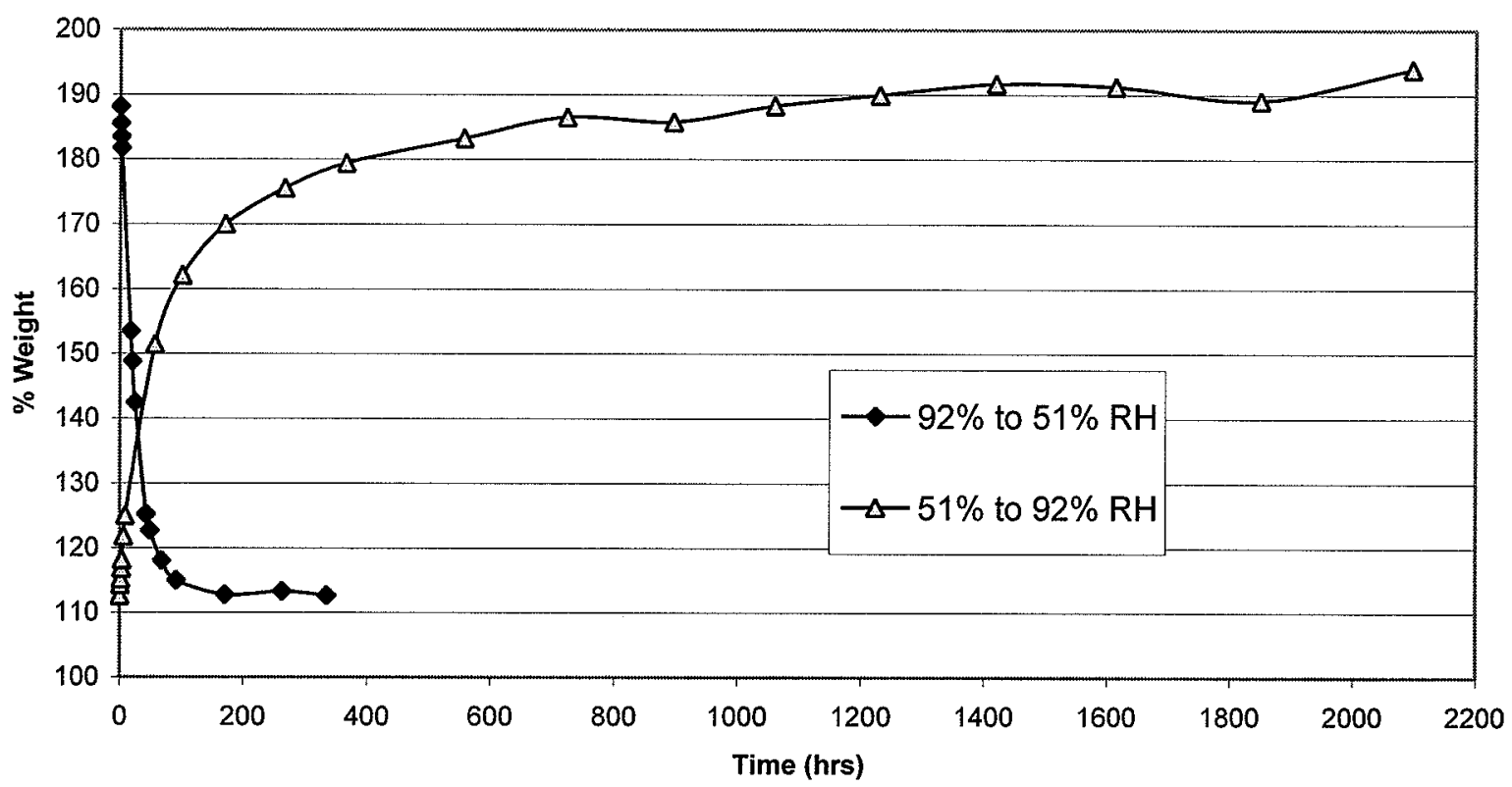

Figure 11: The uptake and release of water vapour by nano-structured calcium silicate cycled between relative humidity environments of $92 \% \mathrm{RH}$ and $51 \% \mathrm{RH}$ respectively. 
Nectarines after 18 days

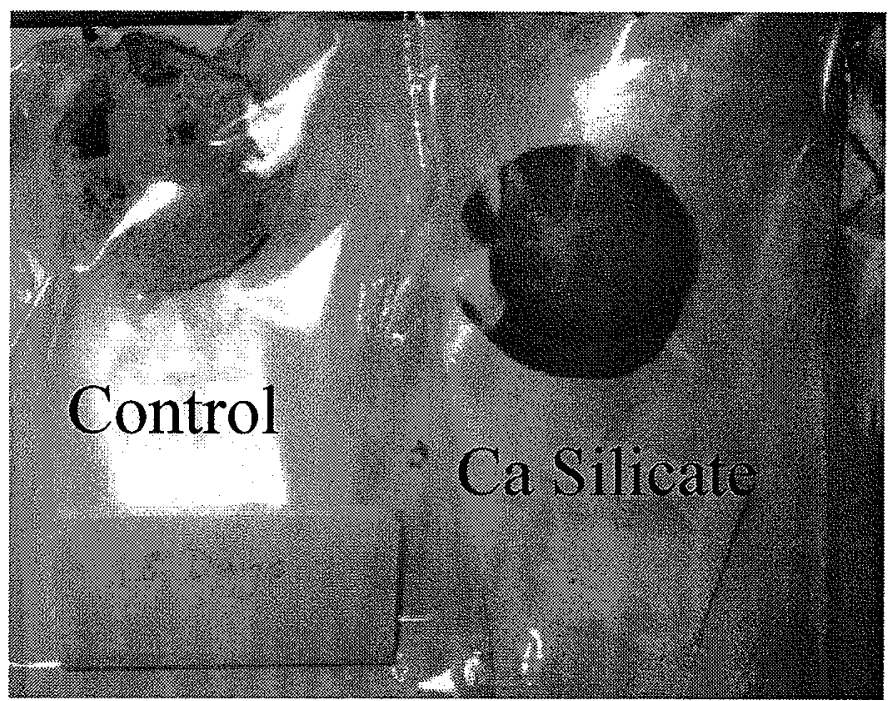

Pears after 20 days

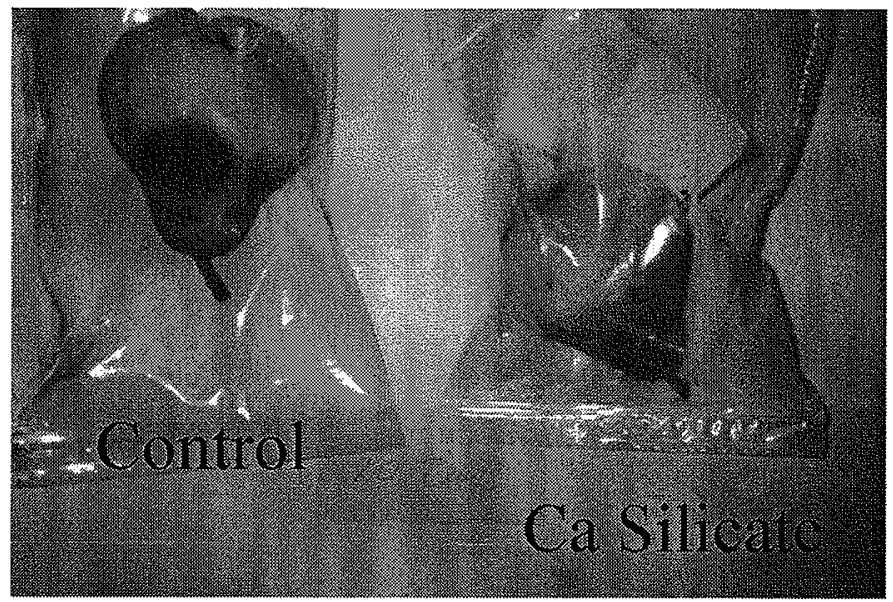

Figure 12: The effect of nano-structured calcium silicate on controlling the ripening of fruit and extending the shelf life. 

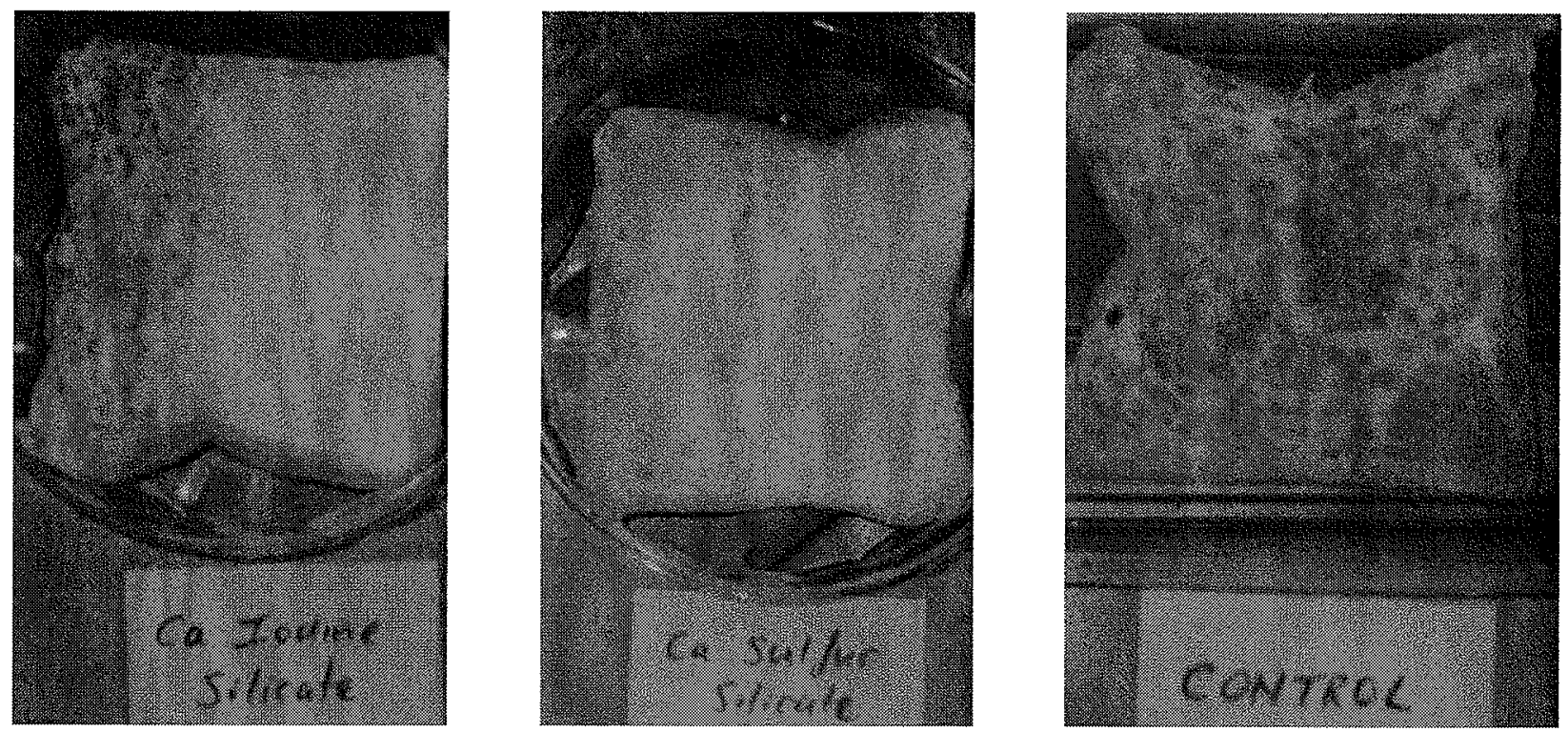

Figure 13: The anti-microbial action of nano-structured calcium silicate composites with iodine and sulfur on bread substrates which have been exposed to an environment conducive to mould growth for 10 days. The silicate is sprinkled on the right hand side of the bread. The control sample contains no silicate. 